\title{
MATCHED-FIELD SOURCE DETECTION AND LOCALIZATION \\ IN HIGH NOISE ENVIRONMENTS: , A NOVEL REDUCED-RANK SIGNAL PROCESSING APPROACH
}

\section{A Dissertation Presented to}

The Faculty of the College of Engineering and Technology

Ohio University

\author{
In Partial Fulfillment \\ of the Requirements for the Degree \\ Doctor of Philosophy \\ by

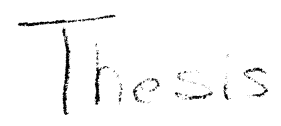 \\ 1 \\ 1914 \\ H. Bryan Riley, \\ March, 1994




\section{ACKNOWLEDGEMENTS}

I would like to express my appreciation to Dr. John A. Tague for his efforts and patience in directing my activities throughout this research project. Successful completion of this work would not have been possible without his guidance and counseling.

I would also thank the members of the dissertation committee for their helpful insights. These members were: Drs. Sunil Agrawal, Jeffery Dill, Joseph Essman, and Jeffery Giesey. I thank Dr. Roger Radcliff, my initial contact, for the support provided during my studies at Ohio University. I am also grateful for both the support and the resources provided by the Department of Electrical and Computer Engineering.

I thank Dr. Ivars Kirsteins for helpful technical discussion in the area of reduced rank signal processing and Dr. Mike Collins for helpful technical discussions in the area of underwater acoustical modeling. I am indebted to your willingness to answer my questions and to offer suggestions when I needed help.

Special acknowledgements go to Drs. Harold Martin and Samual White who I admire greatly. These two men have been very positive role models and mentors to me.

I am further grateful to Mr. John Link for help to blaze the opportunity for me return to school, for the support, and for the resources that were made available through Magnavox Electronic Systems Company. His frankness is appreciated and shall never be forgotten.

Finally, I thank my family. Thanks to both my parents and my parents-in-law for their unconditional love and support. I thank my little daughter Brittany for her display of innocence. She gives joy into my life. I especially thank my lovely wife, San, who has provided me with love, support, and encouragement throughout my graduate studies. She is the person with whom I have already made my best discoveries. 
TABLE OF CONTENTS

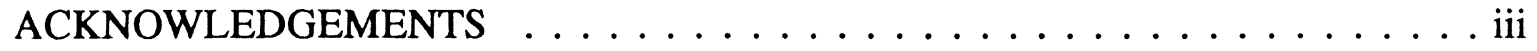

TABLE OF CONTENTS $\ldots \ldots \ldots \ldots \ldots \ldots \ldots \ldots$

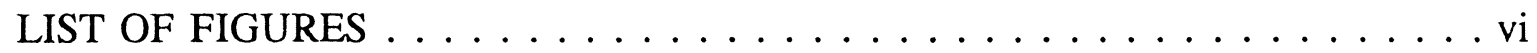

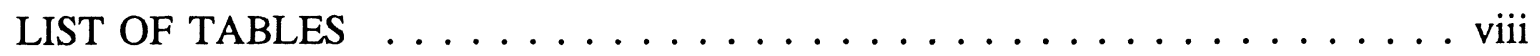

LIST OF SYMBOLS AND ABBREVIATIONS ............... ix

CHAPTER

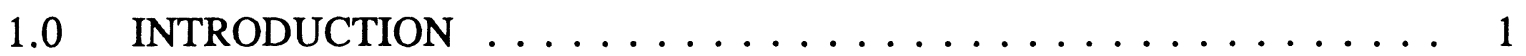

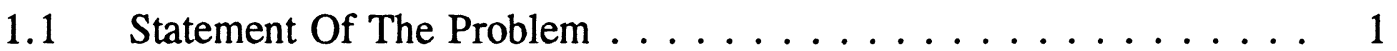

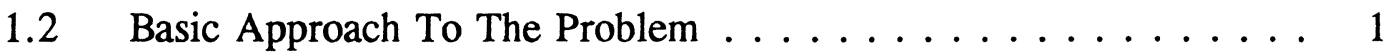

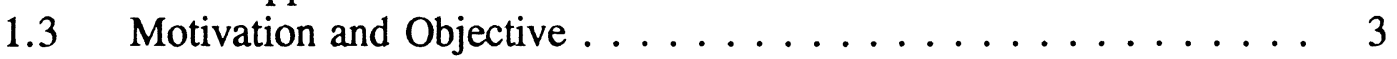

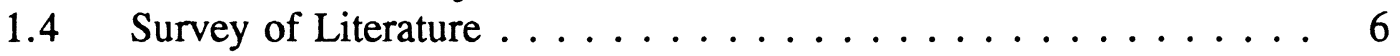

1.5 Overview of the Dissertation $\ldots \ldots \ldots \ldots \ldots$

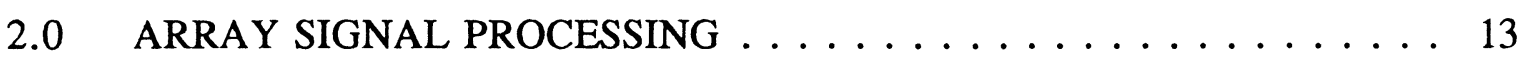

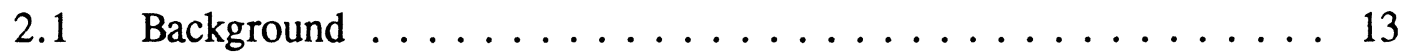

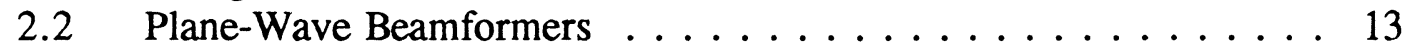

2.2.1 Basic Concepts and Terminology ............ . . 14

2.2.2 Frequency-Wavenumber Space ............. . . 20

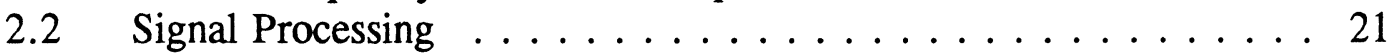

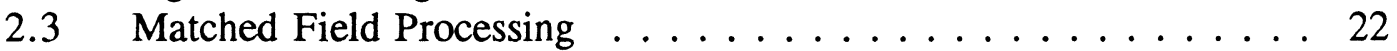

2.4 Conventional Source Position Estimators . . . . . . . . . . 26

2.4 .1 The Bartlett Estimator . . . . . . . . . . . . . . 26

2.4.2 The Maximum Likelihood Estimator . . . . . . . . . 27

3.0 ACOUSTIC MODELING OF OCEAN ENVIRONMENTS . . . . . 30

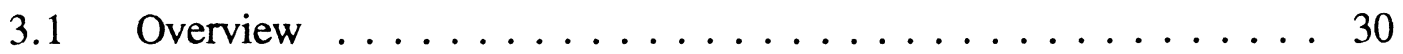

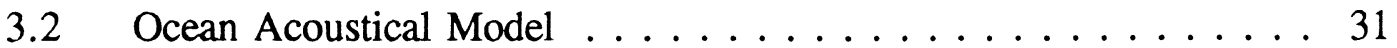

3.3 Wave Theory ........................... 34

3.3.1 Normal-Mode Solutions to the Wave Equation ....... 37

3.3.2 Parabolic Equation Method ... . . . . . . . . . . . . 42

3.4 Acoustic Data Simulation .................. 45

4.0 REDUCED-RANK SIGNAL PROCESSING APPLIED

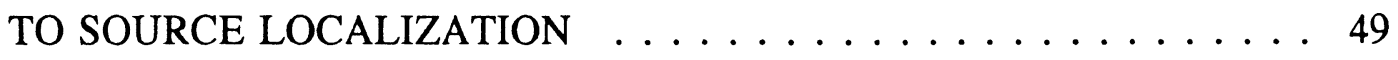

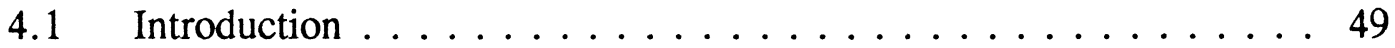


4.2 Background ................... 50

4.3 Signal and Noise Models . . . . . . . . . . . . . . . 51

4.4 Derivation of The Generalized Likelihood Ratio Detector . . . . . 55

4.5 GLRT Performance Evaluation In An Infinite, Homogeneous

Medium .....................663

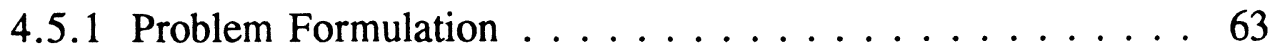

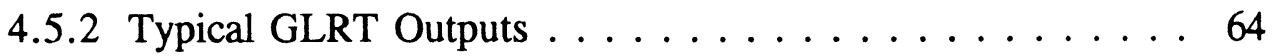

4.5.3 GLRT As A Detector . . . . . . . . . . . . 74

4.5.4 The GLRT As A Direction-of-Arrival Estimator . . . . . . 81

4.6 GLRT Maxima Analysis . . . . . . . . . . 83

5.0 SIMULATIONS FOR MATCHED-FIELD SOURCE LOCALIZATION . 89

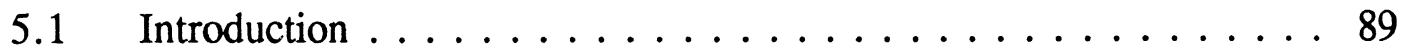

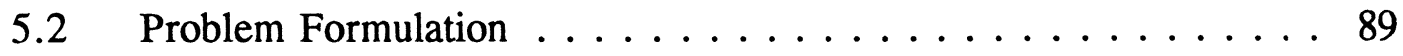

5.3 The Bartlett Estimator . . . . . . . . . . . . . . 92

5.4 The Maximum Likelihood Estimator . . . . . . . . . . . . . . . 97

5.5 The Generalized Likelihood Ratio Test . . . . . . . . . . . . 101

5.6 Summary of Simulations . . . . . . . . . . . . 106

5.7 Critical Evaluation Of The GLRT . . . . . . . . . . 107

6.0 CONCLUDING REMARKS $\ldots \ldots \ldots \ldots \ldots$

6.1 Conclusions . . . . . . . . . . . . . . . . . 108

6.2 Suggestions for Future Research . . . . . . . . . . 110

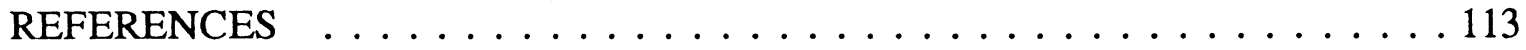

APPENDIX A The Parabolic Equation Derivation $\ldots \ldots \ldots \ldots \ldots$

APPENDIX B Parabolic Equation Solver Code . . . . . . . . . . . . 126

APPENDIX C DOA Estimation Algorithm Code . . . . . . . . . . 143

APPENDIX D $\quad$ ROC Computational Code $\ldots \ldots \ldots \ldots \ldots \ldots$

APPENDIX E Modified GLRT Code To Investigate Subspace Decomposition . 149

APPENDIX F P/E Solver, Bartlett, and MLM Computational Code . . . . . 153

APPENDIX G Bartlett and MLM Ambiguity Surface Display Code . . . . . 170

APPENDIX H GLRT Computational and Display Code . . . . . . . . 172

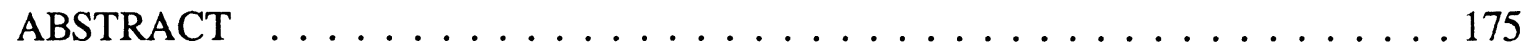




\section{LIST OF FIGURES}

Figure 1.1 A Matched Field Processing Methodology . . . . . . . . . . . . 4

Figure 2.1 A Typical Narrowband Beamformer $\ldots \ldots \ldots \ldots \ldots$

Figure 2.2 Layered Waveguide. . . . . . . . . . . . . . . 23

Figure 3.1 Ocean Environment Model . . . . . . . . . . . 32

Figure 3.2 The Modified PE Solver $\ldots \ldots \ldots \ldots \ldots$

Figure 4.1 DOA Case 1: Weak source distantly space from a strong jammer. . 66

Figure 4.2 DOA Case 2: Strong source distantly spaced from a weak jammer. . 67

Figure 4.3 DOA Case 3: Weak source closely spaced to a strong jammer. . . . 69

Figure 4.4 DOA Case 4: $\mathrm{H}_{\mathrm{O}}$ Assumption - Detection of a weak jammer. . . . 70

Figure 4.5 DOA Case 5: Weak source very closely spaced to a strong jammer. 72

Figure 4.6 DOA Case 6: Weak source very closely spaced to a strong jammer. . 73

Figure 4.7 ROC Case 1: The jammer is located at 8 degrees. . . . . . . . 77

Figure 4.8 ROC Case 2: The jammer is located at 8 degrees. . . . . . 78

Figure 4.9 ROC Case 3: The jammer is located at 8 degrees . . . . . . . . 79

Figure 4.10 ROC Case 4: The jammer is located at 5 degrees $\ldots \ldots$. . . 80

Figure 4.11 GLRT output as replica spans field of view $\left(\mathrm{H}_{1}\right.$ assumption) $\ldots . .86$

Figure 4.12 GLRT output as replica spans field of view $\left(\mathrm{H}_{\mathrm{o}}\right.$ assumption) $\ldots$. . 86

Figure 4.13 Replica vector projected on to signal subspace $\ldots \ldots$. . . . 87

Figure 4.14 Replica vector projected on noise subspace $\ldots \ldots \ldots$. . . . 87

Figure 4.15 Changes in largest eigenvalue under $\mathrm{H}_{1}$ assumption $\ldots \ldots 88$

Figure 4.16 White noise estimate under $\mathrm{H}_{1}$ assumption $\ldots \ldots \ldots 88$ 
Figure 5.1 Geoacoustics of the environment used for simulations . . . . . . . 91

Figure 5.2 MFP Case 1: Bartlett Ambiguity Surface, No Thresholding . . . . 95

Figure 5.3 MFP Case 2: Bartlett Ambiguity Surface, Threshold $=1.1 \ldots 95$

Figure 5.4 MFP Case 3: MLM Ambiguity Surface, No Thresholding . . . . 99

Figure 5.5 MFP Case 4: MLM Ambiguity Surface, Threshold $=0.2 \ldots \ldots 100$

Figure 5.6 MFP Case 5: GLRT Ambiguity Surface, Source Plus Jammer, Threshold $=0.0 \ldots \ldots \ldots \ldots \ldots \ldots \ldots \ldots \ldots$

Figure 5.7 MFP Case 6: GLRT Ambiguity Surface, Source Plus Jammer, Threshold $=500 \ldots \ldots \ldots \ldots \ldots \ldots$

Figure 5.8 MFP Case 6: GLRT Ambiguity Surface, Low Rank Jammer,

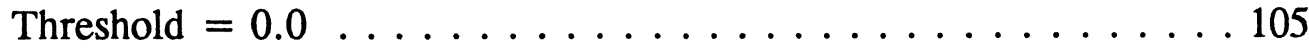




\section{LIST OF TABLES}

Table 4.1 Direction Of Arrival Parameter Estimation Summary . . . . . . . 81

Table 4.2 White Noise Estimates $\ldots \ldots \ldots \ldots \ldots \ldots$

Table 5.1 Summary of Estimated $(\mathrm{r}, \mathrm{z})$ Parameters $\ldots \ldots \ldots \ldots \ldots$ 


\section{LIST OF SYMBOLS AND ABBREVIATIONS}

\section{Principal Symbols}

vector (i.e, a, $\boldsymbol{\phi}$ ) bold-faced, lower-cased Greek or Roman letter

matrix (i.e., $\mathbf{A}, \mathbf{\Phi}$ ) bold-faced, upper-cased Greek or Roman letter

$\beta_{\mathrm{m}} \quad$ vertical component of wavenumber in the water later for the $m$ th mode

$\mathrm{k}_{\mathrm{m}} \quad$ horizontal component for the wavenumber for the mth mode

$\beta \quad$ modulus of elasticity

d distance (meters) between two sensors

$\mathrm{E}\{\cdot\} \quad$ mathematical expectation

f

frequency $(\mathrm{Hz})$

$\mathrm{H}$

Hermitian, complex conjugate transpose

j

$\sqrt{-1}$

$\mathrm{k}$

wavenumber

$\mathrm{N}$

number of elements

$n(t), s(t)$

time dependent variables

$\alpha_{\mathrm{w}}, \alpha_{\mathrm{s}}, \alpha_{\mathrm{b}}$

attenuation in the water, sediment, and subbottom respectively

$\rho_{\mathrm{w}}, \rho_{\mathrm{s}}, \rho_{\mathrm{b}}$

densities in water, sediment, and subbottom respectively

$\mathrm{c}_{\mathrm{w}}, \mathrm{c}_{s}, \mathrm{c}_{\mathrm{b}}$

sound speeds in the water, sediment, and subbottom respectively

$\mathrm{p}(\cdot)$

probability density function

$r$

horizontal distance of the source from the central element of the array 
Principal Symbols cont.

$\tau$

$\Lambda$

$\eta$

$\mathrm{T}$

$\theta$

$\omega$

^

$\mathbf{u}$

$u_{n}$

$\sigma^{2}$

$\lambda$

Z

$z_{o}$

\begin{tabular}{ll} 
& \multicolumn{1}{c}{ Abbreviations } \\
CFAR & constant false alarm rate \\
DOA & direction of arrival \\
FEPE & finite element parabolic equation \\
FFT & fast Fourier transform \\
GLRT & generalized likelihood ratio test
\end{tabular}

temporal lag variable

the likelihood ratio

threshold for likelihood ratio test

observation time (sec.)

array steering angle measured from the endfire of the array, Azimuthal angle

circular frequency, $(2 \pi f)$

estimate; for example, $\hat{\boldsymbol{R}}$ is the estimate of $\mathbf{R}$

signal output of the line array

signal the $n$th element of the array

variance of a random variable

wavelength

depth of the source below the ocean surface

depth of the array below the ocean surface 
Abbreviations cont.

$\begin{array}{ll}\text { JNR } & \text { jammer-to-noise ratio } \\ \text { MFP } & \text { matched field processing } \\ \text { ML } & \text { maximum likelihood } \\ \text { MLE } & \text { maximum likelihood estimator } \\ \text { MLM } & \text { maximum likelihood method } \\ \text { MMP } & \text { matched mode processor } \\ \mathrm{P}_{\mathrm{D}} & \text { probability of detection } \\ \mathrm{P}_{\mathrm{FA}} & \text { probability of false alarm } \\ \mathrm{PDF} & \text { probability density function } \\ \mathrm{PE} & \text { parabolic equation } \\ \mathrm{ROC} & \text { receiver operating characteristics } \\ \mathrm{SNR} & \text { signal-to-noise ratio } \\ \text { TL } & \text { transmission Loss }\end{array}$




\section{CHAPTER 1 \\ INTRODUCTION}

\subsection{Statement Of The Problem}

This dissertation investigates underwater acoustic source detection and localization in the context of matched field processing (MFP). Matched field processing refers to a variety of underwater signal processing methods used to estimate the location of an acoustic source. We develop a Generalized Likelihood Ratio Test (GLRT) which deals with several limitations encountered in conventional MFP. Much of the research in the MFP community has focused on increasing the degree of accuracy of models to represent realistic underwater environments, and this is vitally important. However, in this work, we build on the latest acoustic modeling developments and focus on improving the signal processing methodologies. This investigation is warranted because conventional signal processing methods can not effectively suppress strong directional interference. Typically, this results in a high degree of ambiguity in the estimated source position. In this dissertation, we show how to incorporate directional interference into the MFP scheme, estimate its contribution, and remove it from the data using novel statistical estimation techniques.

\subsection{Basic Approach To The Problem}

To accomplish the above task, we develop a new signal processor that can give superior results when interference is present. Adaptive interference nulling while localizing a source in a noisy underwater environment has been studied a great deal, and results are documented in many publications [1],[2],[3], and [4]. A common approach to detecting and localizing a source is through processing signals measured with an array 
of sensors. This class of signal processors is known as beamformers [5]. Beamformers are the signal processing subsystems of SONAR* (Sound Navigation and Ranging) systems. Conventional beamforming requires at a minimum an array of sensors and that we impose "plane wave propagation" assumptions on the underwater environment. However, this traditional method can be adversely affected if the source field departs markedly from a plane wave due to correlated vertical structures, and bottom surface interactions.

In MFP, an attempt is made to characterize the field structure in the medium more accurately. We accurately model the structure of the environment and imprecision of the data measurements. Limitations of the matched field processor, which exploits the propagational physics, as well as those of the beamformer offer an opportunity to develop more robust signal processing algorithms which estimate and remove "jammer-like" sources in conjunction with detecting the source of interest. We align this investigation with those currently conducted by the United States Naval Research community by modeling shallow water oceans and assuming far-field source radiation conditions. Several primary ideas that we apply are derived from the statistical literature. We show, both by theoretical arguments and by simulations that MFP can be successfully accomplished in high noise environments.

"SONAR, in this context, is a passive system that is designed to separate spatially propagating signals from a particular direction while simultaneously nulling spatially propagating signals from undesired directions. Although the signals may originate from different spatial directions they may have overlapping frequency content. 
In this investigation, we incorporate the use of underwater sound propagation theory and well known spectral estimators to gain insight into MFP. After establishing the limitations of conventional methods, we demonstrate the viability of this new signal processing methodology which imposes structure on the interference and adaptively estimates it for subsequent removal. We show that these developments in the signal processing component of MFP provide superior source position estimates.

\subsection{Motivation and Objective}

This dissertation presents a new approach to MFP based on several well known principles as well as a generally accepted implementation philosophy. Figure 1.1 illustrates the relationship between these principles and the generalized implementation structure for MFP. The major implementation components indicated in the rectangular outlines of Figure 1.1 are:

1) a propagation model which can be parameterized for realistic ocean environments;

2) data measurements of the incident field produced by the source; and,

3) a signal processor which extracts localization information by processing the data. Inputs to the generalized MFP system influence performance and are described.

The acoustic model requires an accurate characterization of the physical phenomenology and a definition for the assumed signal model and noise model. Concepts from detection theory, estimation theory, linear algebra and statistics are the tools used to develop the GLRT which is the heart of the signal processing. 


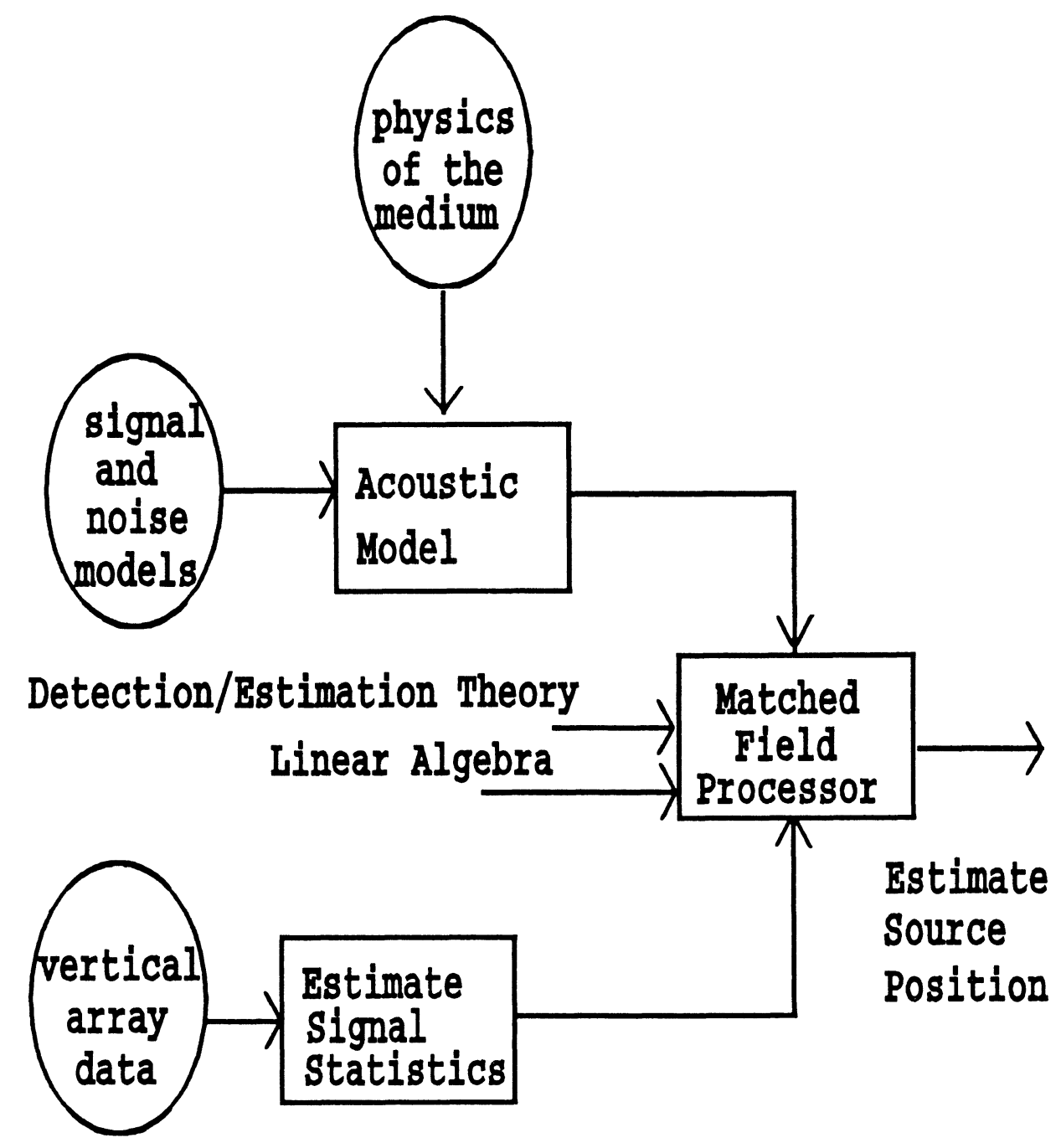

Figure 1.1 A Matched Field Processing Methodology 
From a signal processing point of view the following issues are taken into account:

1) incorporate directional interference into the MFP scenario;

2) model range dependent environments where the plane wave assumption is not valid;

3) account for sensitivity to imprecise knowledge of the environment and sensor characteristics; and

4) consider computational complexity and processor loading

In practice, MFP is influenced by an accurate characterization of environmental information. Continuous research, by acousticians, of complicated media results in improvements to ocean acoustical models. These models predict sound pressure with increasing accuracy and drive the requirement for more sophisticated signal processing.

The objectives and contributions of this dissertation are summarized below.

1) Detect the presence of a signal of interest. Specifically we demonstrate the ability to detect a deterministic signal in strong low rank Gaussian interference plus white noise.

2) Estimate the range and depth coordinates which localize the source in high noise environments.

3) Incorporate more realistic characterizations of ocean noise into the problem. The noise is modeled such that it contains both white noise and directional interference.

4) Estimate directional interference, and remove it from the array data prior to signal processing for target localization.

5) Exploit concepts from linear algebra, such as the approximation of a data matrix by another of lower rank, and decomposition of a measurement space into a signal subspace and a noise subspace.

6) Perform experiments which demonstrate the validity of this approach. 


\subsection{Survey of Literature}

This section presents a brief review of the MFP literature. This research topic is conducted primarily from an underwater acoustics point of view. The literature does not indicate many contributions from a signal processing perspective.

We begin with a presentation of work from pioneer investigators such as Bucker, Clay, and Hinick [6], [7], and [8]. We proceed with a review which includes application of state of the art tools such as neural networks for intelligent replica field selection. Many of the current contributions in the matched field processing community are based on initial work conducted by Bucker. Bucker proposed an algorithm for localizing a noise source by comparing the measured cross-spectral matrix elements different target locations (1976). The cases investigated contained a significant degree of difficulty and the results showed promise for the proposed method. He suggested additional detailed experiments for further validation.

Bucker made calculations for a sound field in a shallow water duct and compared them to the field data. He found the normal mode representation of the sound field for a channel with lossy boundaries. The sound velocity profile was assumed to vary only in one direction. Equations were derived for efficient calculation of sound levels in a duct or waveguide with lossy boundaries. A comparison was made between calculated and experimental values for propagation loss. Bucker found reasonable agreements using surface losses values of $0 \mathrm{~dB}$ and $1 \mathrm{~dB}$ attenuation.

Clay conducted several MFP investigations using arrays of sources and receivers as filters in waveguides. He examined the transmission between an array of sources and 
an array of receivers to investigate the different modes with phases and amplitudes that depend on the depth of a source. Normal mode theory and stratified waveguides were used to analyze the signal-to-noise gain of a vertical hydrophone array in a noisy ocean. Clay's work concluded with a formal solution for a radiation field in a waveguide. Clay investigated steering an array in a waveguide. He investigated propagation of bandlimited low frequency noise in shallow water.

Hinich is credited with the "maximum likelihood" method of source depth localization. Data from a vertical array is processed using the maximum-likelihood estimator (MLE) to estimate the source depth. The noise is assumed to be Gaussian. The MLE of the source function is estimated by the linear combination of $M$ eigenfunctions

$$
\hat{s}(x)=\sum_{m=1}^{M} \hat{\theta}_{m} \psi_{m}(x)
$$

where $\left\{\hat{\theta}_{m}\right\}$ are estimates of the unknown weights whose values are such that $\mathbf{s}(\mathbf{x})$ has a global maximum at the source $\left(x=x_{s}\right)$, and $\psi_{\mathrm{m}}$ are the modes of the source. Hinich's work showed that given the eigenfunctions, the maximum-likelihood signal processing technique can be applied to any waveguide in which a source is detected by an array of sensors.

Much of the earlier work described above was performed using the well known Pekeris model [9]. This model assumes a two layered ocean. Pekeris conducted the first quantitative analysis of low-frequency sound propagation. He used the method of normal 
modes to obtain a far field solution and applied it to an explosive point source. Tolstoy studied extensively and contributed to the theory of acoustic wave propagation from a simple point source in a layered waveguide [10].

Many MFP methodologies have been developed by Klemm, Baggeroer et. al., Schmidt et. al., Ozard, and Feuillade et. al. [11], [12], [13], [14], and [15]. Baggeroer et. al. applied the Cramer-Rao formalism to establish a lower bound on the resolution obtainable for matched field source localization. Candy and Sullivan used a model-based approach in conjunction with the normal mode method to estimate the wavenumbers from the pressure measurements. A passive ranging scheme utilizing the wavenumbers was developed. Candy demonstrated that no a priori knowledge of the model parameters is required to obtain accurate estimates of the wavenumbers [16].

Several recent papers have appeared in the literature where the robustness of this processing method has been investigated. Hamson and Heitmeyer have investigated the affect of sediment compressional wave speed on matched field processing [17]. They conclude that large errors in the compressional wave speed of the bottom did not effect the ability of MFP to predict the location of the source.

The direct inversion or Matched-Mode Processing (MMP) was developed by Shang and refined by Yang [18] and [19]. In this work, a modal pressure field processor is designed that allows estimation of the source position. The range information and depth information are separable in mode space. A propagation model, usually the normal mode model, models the modal structure. The MMP approach is based on the fact that the normal mode model produces a set of equations which can be inverted. The 
number of modes needed to determine range and depth depends on the sound channel, the array aperture, and the transmission frequency. The matching procedure can be carried out using a subset of the total resolved modes which avoids certain kinds of environmental mismatch conditions. Yang reports results on the sensitivity of varying the number of modes used for estimates of the source position.

The work of Zala and Ozard presented MFP results for range-independent and range-independent environments [20]. More than 100 cases using four array configurations and 3 processors were utilized. It was determined that range-dependent models in MFP significantly improved array design and analysis of real world localization problems.

Ozard et. al. developed a nearest-neighbors algorithm as an intelligent method of selecting replicas to speed up MFP. A description of the selection process is outlined and the performance of their neural network is compared to the unnormalized Bartlett beamformer [21].

Porter et. al. simulated a deep-water environment to study mismatch between replica and actual fields [22]. The impact of mismatch caused by uncertainties in the hydrophone positions, bottom parameters, and sound speed were criterion used to examine the performance of several power estimators.

A major problem associated with MFP is the difficulty of maintaining the hydrophone array vertical and at a constant depth during the experiment. Stergiopoulos developed an algorithm for extended horizontal measurements and demonstrated its effectiveness [23]. 
Byrne et. al. applied Capon's maximum likelihood method (MLM) to estimate the range and depth of an acoustic source [24]. The MLM offers the capability to adapt to the actual noise rather than requiring an a priori estimate for prewhitening. The algorithm was determined to be unstable and several cases were examined to determine sensitivity.

Collins and Kuperman developed a generalized approach to MFP called focalization [25]. Focalization requires that the environment is treated as an acoustic lens. An attempt is made to simultaneously localize the source and estimate the ocean acoustic parameters. The ocean acoustic parameters are varied in order to localize the source with a high-resolution cost function. It was determined that the focalization localizes source location before the ocean acoustic parameters. An iterative procedure follows which then estimates the ocean acoustic parameters in addition to localizing the source.

Richardson and Nolte incorporated environmental uncertainty in the processor design via probability density functions (PDF) [26]. The optimal uncertain field processor or OUFP is a algorithm that compensates for uncertainty in the acoustic environment. It was developed utilizing parameter estimation theory and shown to be less sensitive to mismatch between the assumed and actual environments.

Minimax robust processing methods were applied to matched-field processing by Gingras and Gerr [27]. They investigated the performance of the robust processor in the presence of uncertain environmental information and compared it to conventional methods. Secondly, Gingras and Gerr developed channel model-based array processing 
methods based on well established detection theory. Basic assumptions about the signal and noise processes and acoustic channel properties were applied. A simulation which illustrates the application of the minimax methods in the context of shallow-water was produced.

\subsection{Overview of the Dissertation}

This dissertation is organized in the following manner. Chapter 2 provides an overview of matched-field processing for source localization. We start with background information from which this MFP investigation is formulated. This requires that we describe the essential components of beamforming of array data for source localization. We introduce two common estimators and define them in the context of MFP. Chapter 2 includes a survey of previous work published in the literature. In chapter 3 , the essential mathematical equations to model ocean acoustic propagation are presented. An overview of underwater acoustic propagation theory is present. We continue with a description of two distinct methods to solving the Helmholtz equation which governs underwater sound propagation and derive a representative complex pressure equation for a range dependent environment. Chapter 4 presents a description of the signal processing applied to source localization. This chapter begins with the background and theory of reduced rank signal processing. The central theme of this chapter is the derivation of a Generalized Likelihood Ratio Test (GLRT). This chapter describes several GLRT outputs, simulations of the GLRT as a detector, and as a direction-of-arrival (DOA) estimator. We conduct an analysis to determine why the output of the GLRT peaks 
which indicates localization of the source. Chapter 5 presents representative results for several specific localization scenarios and compares the results produced by the Bartlett Estimator and Maximum Likelihood Estimator to the GLRT output. A critical evaluation of these experiments is presented. Chapter 6 presents the conclusions obtained from this research and suggestions for future research. 


\section{CHAPTER 2 \\ ARRAY SIGNAL PROCESSING}

\subsection{Background}

This chapter reviews a component of array signal processing required for source localization termed "beamforming". Beamforming is the signal processing method of spatially filtering multisensor data to form beams for the reception of energy [28].

The basic assumptions and limitations required to localize a source by processing the field received on a vertical array are discussed. We then describe the generalized matched field beamformer as a processor that "matches" the measured acoustic field at the array with replicas of the expected field for all source locations.

\subsection{Plane-Wave Beamformers}

The beamformer is a spatial filtering system designed to separate signals that have overlapping frequency content. The signals may originate from different bearing or spatial locations. A vertical line array with equally spaced sensors is used to measure the ambient noise as well as properties of signals arriving from different directions. Transducers convert the acoustical energy into electrical energy. Measurement vectors or "snapshots" from the vertical array are the data used for processing. The directions from which underwater signal propagate can in principle be extracted from this data. As a problem of practical interest, we concentrate on cases in which the number of arrival directions does not exceed the number of receivers. The processor shall operate on an array covariance matrix and the goal is to estimate the power from the $N$ directions. This processing method assumes homogeneous wavefields which are the superposition of uncorrelated elementary waves. This process modifies outputs from an array of 
sensors to enhance desired signal reception and suppress background noise along with undesired signals.

Common assumptions involved in conventional beamforming are:

1) the array does not distort the signal field;

2) the signals are homogeneous when in proximity to the array;

3) the signals are zero mean, Gaussian random processes; and,

4) the sensitivity of each receiver is known and accounted.

The conventional beamformer is commonly used due to its efficient computational implementation with Fast Fourier Transforms (FFTs) and its robustness. To explain this processing methodology, the conventional (time-delay and sum) beamformer is derived and illustrated as an example. Numerous publications are devoted to the technological developments in beamforming for array applications in the signal processing literature $[5],[29]$, and [30].

\subsubsection{Basic Concepts and Terminology}

The signals from the sensor outputs are passed through a receiver where they are amplified, shifted to baseband, lowpass filtered, sampled, and digitized. The array is essentially a spatial sampler. As such we define the sampling requirements for analysis similar to those of the temporal process. We are always working with bandlimited signals which arise naturally through propagation in the medium. They are strictly bandlimited due to the fixed upper limit of $2 \pi / \lambda$ for a maximum value of the wave number or spatial frequency. $\lambda$ is the signal wavelength. The first stage produces a vector of sampled outputs for beamforming. In its elementary form, the plane wave 
beamformer performs spatial filtering of discrete spatially sampled data. The beamformer linearly combines the spatially sampled data from each sensor to obtain a scaler output of the original times series. Through application of appropriate phase delays, the desired signal combines coherently and the noise adds incoherently. The assumption that a plane wave traverses the array states at any time $t$, a propagating signal received anywhere on a planer wavefront perpendicular to a line drawn from the source to a point on the plane has equal intensity. Propagation of the signal between two points in space is then completely characterized by delay. This fundamental concept for array signal processing is based upon this plane wave representation for the signal.

The sensor spacing and number of elements define the spatial aperture. As the aperture improves the resolution or discrimination improves. A plane wave is incident on the array at angle $\theta_{\mathrm{i}}$ with respect to the horizontal line of sensors. The appropriate delays or "weights" were applied to each sensor output to compensate for propagation delays forming a sum. The weights are computed for $N$ estimated DOAs because the signal field at the array is comprised of $\mathrm{N}$ independent plane wave arrivals from known directions. Figure 2.1 indicates this typical narrowband beamformer.

The time domain implementation is the well known sum-and-delay beamformer and is defined as

$$
y(t, \theta)=\sum_{n=0}^{N-1} w_{n} x_{n}\left(t-\tau_{n}\right)
$$

where $\mathbf{x}_{\mathrm{n}}(\mathrm{t})$ is the signal at the $\mathrm{n}$ th sensor and 


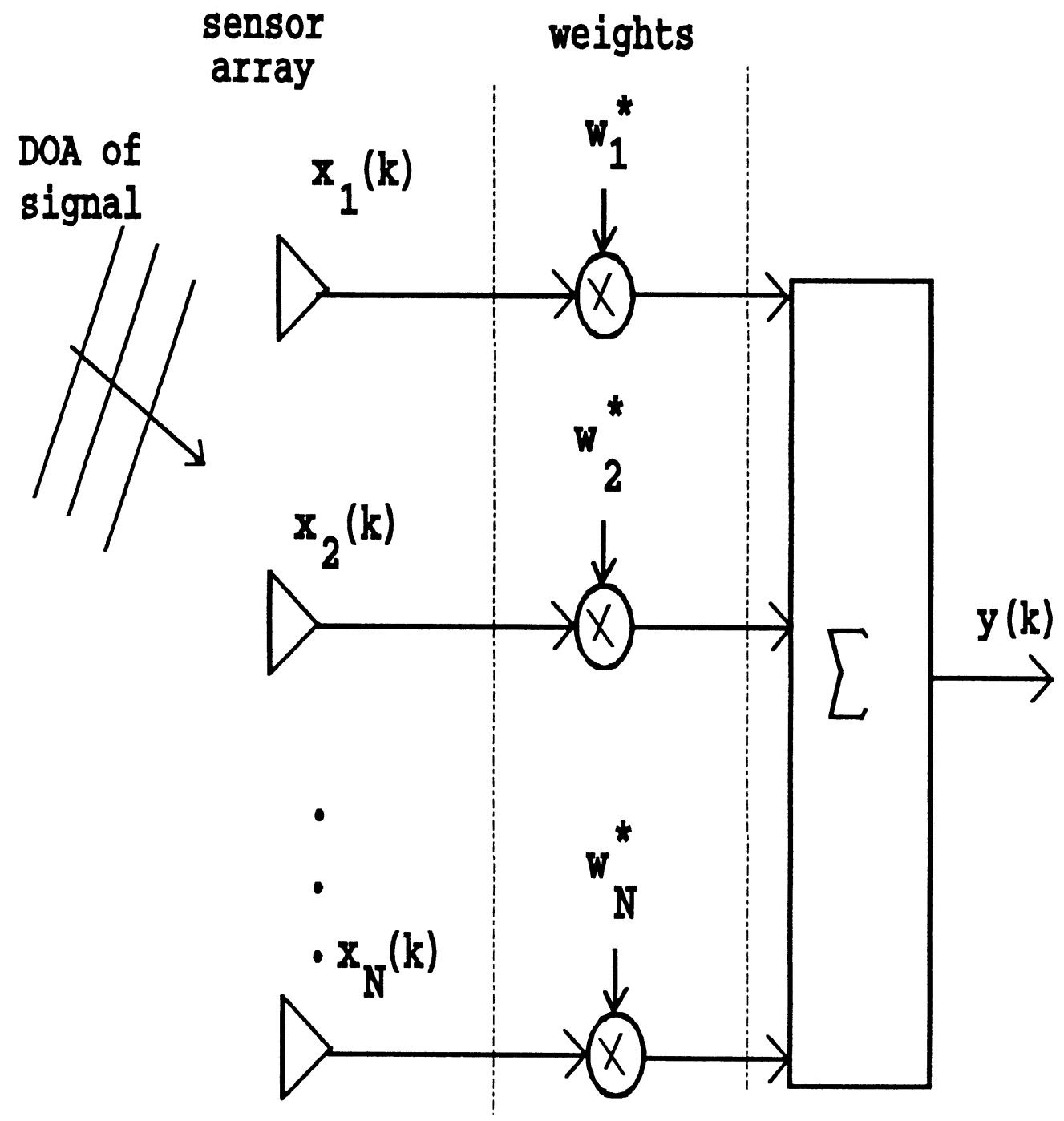

Figure 2.1 A Typical Narrowband Beamformer 


$$
\tau_{n}=n d \sin \theta / c \quad ; \quad n=0,1, \ldots, N
$$

is the time delay. Note that the time delay $\tau$ is a function of sensor spacing $d$, arrival angle $\theta$, and sound speed propagation in water $c$. The amplitude weights $\left\{a_{n}\right\}$ are usually chosen according to the criterion of steering the beam in a particular direction. This is a significant advantage in that changes can be easily implemented to maintain suppression of interfering signals.

The sum-and-delay operation has an equivalent frequency domain representation which can be derived by taking the Fourier transform of the output $y(t)$

$$
Y(\omega, \theta)=\sum_{n=0}^{N} y_{m}(\omega) a_{m} e^{-j \omega \tau_{n}}=A^{H} \boldsymbol{X}
$$

where $\mathbf{A}$ and $\mathbf{X}$ are $N x 1$ dimensional vectors representing the filter transfer functions and the Fourier spectra respectively. The superscript $\mathrm{H}$ denotes the Hermitian operation and $\mathbf{A}$ and $\mathbf{X}$ can be written as 


$$
\boldsymbol{A}=\left[\begin{array}{c}
a_{o} e^{-j \omega \tau_{0}} \\
a_{1} e^{-j \omega \tau_{1}} \\
\cdot \\
\cdot \\
a_{n-1} e^{-j \omega \tau_{n}}
\end{array}\right] \quad, \boldsymbol{X}=\left[\begin{array}{c}
X_{0}(\omega) \\
X_{1}(\omega) \\
\cdot \\
\cdot \\
X_{n-1}(\omega)
\end{array}\right]
$$

This implementation can be accomplished with the fast Fourier transform (FFT) algorithm which operates directly on the data to provide a multidimensional spectral analysis perspective.

The shift and add operation among the sensor outputs result in an SNR improvement by a factor equal to the number of sensors. To show this, let $\mathbf{x}_{\mathrm{i}}(\mathrm{t})$ represent the complex envelope of the total received signal plus noise at the ith sensor:

$$
x_{i}(t)=s(t) e^{-j \pi d s i n(\theta)}+n_{i}(t)
$$

The phase shift factor, $e^{j \pi d_{i} \sin \theta} ; i=1,2, \ldots, N$ provides for the signal components to be combined coherently. The signal and noise models in equation (2.5) are defined in section 2.3.

The input signal-to-noise ratio (SNR) is given as:

$$
(S N R)_{i}=\frac{E\left[\left|s_{i}(t)\right|^{2}\right]}{E\left[\left|n_{i}(t)\right|^{2}\right]} .
$$


The improvement in the signal-to-noise ratio (SNR) at the output can now be defined as:

$$
(S N R)_{o}=\frac{N^{2} P}{\sum_{i} \sum_{j} E\left[n_{i}(t) n_{j}^{H}(t)\right]}=\frac{N^{2} P}{N \sigma^{2}}=N(S N R)_{i}
$$

The quantities $n_{i}$ and $n_{j}$ represent the noise on the $i$ th and $j$ th sensor and $\sigma^{2}$ represents the noise common to all sensors. As a result, the signal adds coherently and noise adds incoherently. Equation (2.7) states that the improvement in SNR is limited by the total number of sensors available in the array. The final result is a gain factor for the overall signal due application of the appropriate phase delays compared to the noise.

A spatial aperture is obtained through discrete sampling by the array of sensors. The degree of resolution of signals depends on the array aperture. That is the Rayleigh resolution, in radians, of the processor is given by $\theta=\lambda / \mathrm{L}$ where $\lambda$ is the acoustic wavelength and $\mathrm{L}$ is the length of the array. Thus, another limitation of conventional plane-wave beamforming with an array occurs when sources are too close together. For example, if two sources are closer than a beamwidth, they can not be distinguished. The ability to distinguish close frequency components requires outputs from a large array. An increase in the aperture produces an increases in discrimination. Spatial coverage is constrained by economic and implementation considerations.

In summary, the conventional beamformer in its simplest processing structure implements a linear regression on the sensor outputs, either in the time domain or frequency domain [5] and [30]. It is convenient to modify the implementation by 
adjusting the weights which the beamformer uses to combine sensor data. The weight adjustments may occur as a function of time which is an advantage when suppressing unwanted signals. MFP offers additional benefits which are obtainable by additional information which describes realistic ocean environments.

\subsubsection{Frequency-Wavenumber Space}

The spatial frequency or wavenumber is an important quantity in the context of array processing. This quantity arises in the signal processing aspect of matched field processing as well as the acoustic modeling and merits a brief discussion.

The acoustic pressure field $\mathbf{p}(t, \mathbf{r})$ is a function of 4 variables: time $t$, and 3 spatial coordinates $\mathbf{r}$, which define the location of the observation point. For sake of this discussion, we take the simple, single frequency plane wave

$$
p(t, r)=e^{j(\omega t-k \cdot r)}
$$

where $\omega$ is radian frequency and $k$ is the wavenumber vector or the spatial frequency variable. The wavenumber vector is predicated on a vector that is normal to the wavefront which is pointed in the direction of propagation. The 3 components of $k$ are referred to as the spatial frequencies or wavenumbers. An arbitrary field $\mathbf{p}(t, \mathbf{r})$ can be represented through its frequency wavenumber transform $F_{p}(\omega, k)$ where $p(t, r)$ is composed of a linear combination of single frequency plane waves. This representation is the four-dimensional Fourier transform of equation (2.8) 


$$
F(\boldsymbol{k}, \omega)=\int_{-\infty}^{\infty} \int_{-\infty}^{\infty} p(\boldsymbol{r}, t) \exp (-j(\omega t-k \cdot r)) d r d t
$$

Just as $\mathbf{t}$ and $\omega$ are duals in the single dimension so are $\mathbf{r}$ and $\mathbf{k}$ duals in the same sense. Therefore by estimating the spectrum, one can determine the presence of a source as well as determine its frequency and direction.

\subsection{Signal Processing}

The signal processing objective is to analyze the multisensor data for estimation of a parameter $\theta_{0}$ of the signal $\mathbf{s}\left(\theta_{0}\right)$ buried in noise. The parameter $\theta$ is varied in a particular power estimator so that the output power becomes maximum. This is the case, for plane wave beamforming, when $\theta=\theta_{\mathrm{o}}$, or the steering vector $\mathbf{e}(\theta)$ coincides with the desired signal. The steering vector contains a priori information of the processor about the desired signal. The quantity of interest is resolution or the degree mismatch between the steering vector $\mathbf{e}(\theta)$ and the observed process $\mathbf{s}\left(\theta_{\mathrm{o}}\right)+\mathbf{n}, \mathbf{n}$ being a noise vector. The signal processing makes use of this a priori knowledge. A radiating point source in the farfield of an isotropic media is modeled as a coherent plane wave. Thus, the incident angle relative to an antenna is a measure of the position of the target.

Power estimators such as the matched filter, discrete Fourier transform and the conventional beamformer can be represented as:

$$
P(\theta)=e^{H}(\theta) R e(\theta)
$$

The input correlation matrix $\mathbf{R}$ is defined in terms of the ensemble average $R \triangleq E\left\{s_{j} s_{j}{ }^{H}\right\}$. This matrix is real, symmetric, positive definite and rarely positive semi- 
definite. $\theta_{\mathrm{o}}$ is the unknown parameter to be estimated. Equation (2.10) may represent the Fourier power spectrum or the power output of either a matched filter or a conventional beamformer, where $\theta$ is frequency, time, or an angle relative to an array, respectively. Equation (2.10) can also be optimized in the signal-to-noise ratio sense if $\mathbf{R}$ consists of a deterministic signal plus noise, i.e., $R=\sigma_{s}^{2} s\left(\theta_{o}\right) s^{H}\left(\theta_{o}\right)+\sigma_{n}^{2} I$.

\subsection{Matched Field Processing}

Matched field processing (MFP), from a signal processing point of view, is a generalized beamformer technique that exploits additional information about the structure of the underwater environment. Here the medium is nonisotropic, such as a waveguide where the field due to a point source is dispersed in different arrivals, and the steering vector has to be matched to the field theoretically by solving the wave equation for a set of boundary conditions or experimentally by use of array measurements. We study an ocean which has a water column of only several hundred meters. An ocean of this depth is referred to as "shallow water". The source localization problem is solved using waveguide models to characterize the shallow water channel. Figure 2.2 illustrates the matched field localization problem which is discussed in the following paragraphs.

MFP is accomplished by using propagation model in conjunction with measured data from an array. The propagation model solves the wave equation given a set boundary conditions which describe the underlying signal and noise structures. In principle, if the propagation characteristics are known, the signal field at each element of the array may be calculated for any source location. Information resulting from the modeling process is combined with sufficient information about the source to compute 


\section{ocean \\ surface}

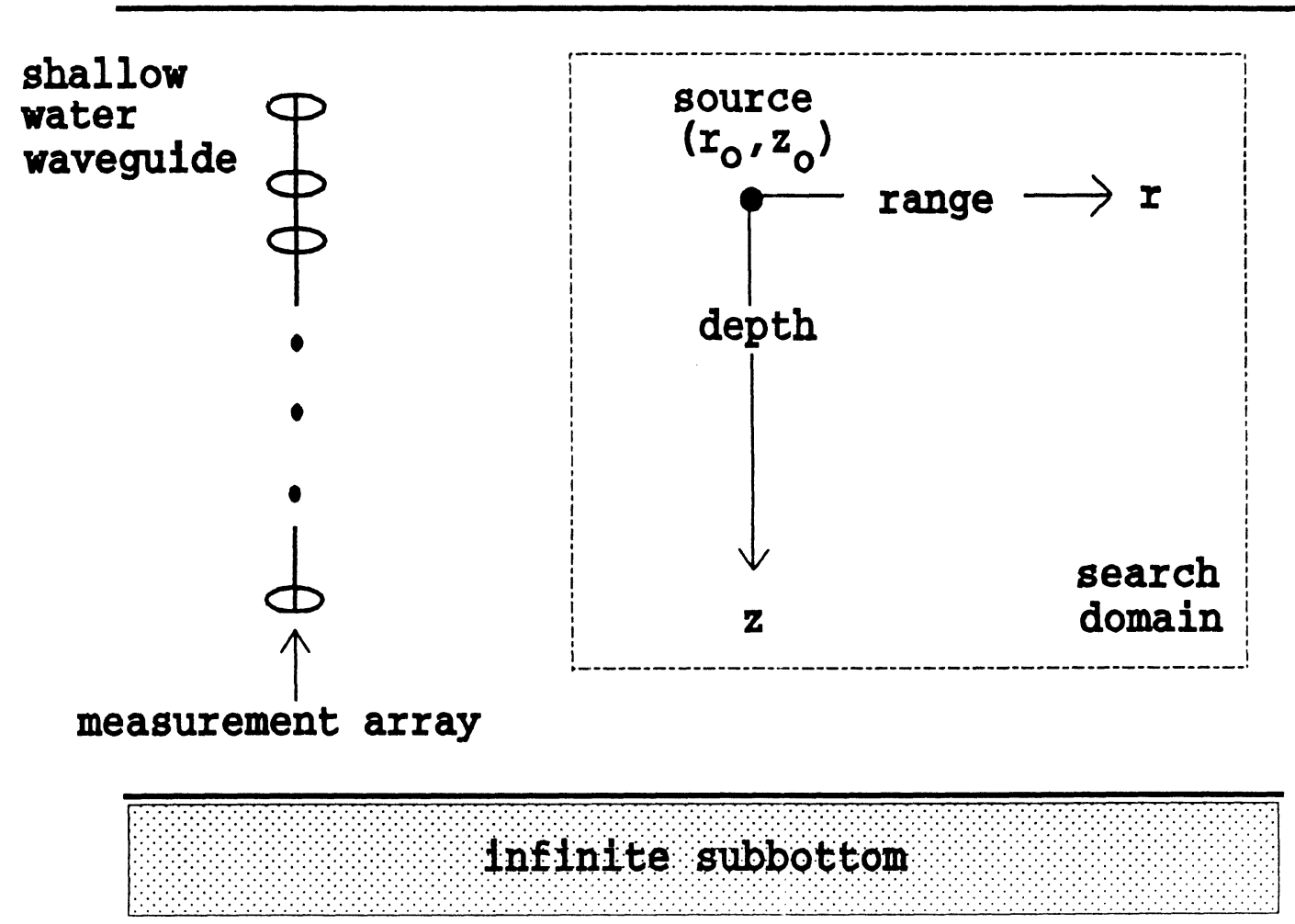

Figure 2.2 Layered Waveguide. Source in a water layer over sediment. The source position varies as $\left(r_{o}, z_{o}\right)$, and the receiver position is fixed. 
the complex acoustic field as a function of source location. This complex acoustic field shall be referred to as the replica field and is indicated by the dotted line in Figure 2.2. It models the recorded complex pressure field recorded at the $n$th element of the a vertical hydrophone array due to the source located at an hypothesized range and depth. The replica field is computed for an exhaustive number of assumed source range and depth locations. This field is incorporated into the array processor. In this way environmental information is included in the processing scheme.

The acoustic pressure field is sampled as a function of the true source position $\theta_{s}=\left(r_{s}, z_{s}\right)$. The pressure vector measured at the array output is given by $\mathbf{x}\left(\theta_{\mathbf{g}}\right)$. Since we have assumed a narrowband source, each sensor output is weighted by a complex matching or "weighing" vector, $\mathbf{w}(\theta)$, which is a function of the unknown source position. The matched field power of the array is simply given by

$$
P(\theta)=w^{H}(\theta) R w(\theta)
$$

where $\theta_{s}=\left(r_{s}, z_{s}\right)$ indicates the true source range, $r_{s}$, and depth, $z_{s}$, and $w\left(\theta_{s}\right)$ is the complex pressure recorded at the array output and normalized to unity. The range and depth are denoted by $r$ and $z$ respectively. The corresponding array output for a given position, $\theta$ is given by

$$
y(\theta)=w^{H}(\theta) x\left(\theta_{s}\right)
$$

where w has dimension $\mathrm{Nx} 1$ and defined by some criterion. Thus, if the same set of environmental parameters are used in the acoustic model for production of the replica field and the "received" field, then there is a "perfect match" and the source can in 
principle be correctly located. Since we are not able to characterize the ocean environmental conditions with precision (mismatch) the processor results contains some ambiguity. Figure 2.2 implies that mismatch as unavoidable in characterizing the realistic ocean.

From a implementation point of view, several issues arise:

1) propagation in a range-dependent environment;

2) imprecise knowledge of the sensor characteristics and propagation conditions;

3) effects usually treated as random such as boundary roughness and internal waves;

4) computational complexity; and,

5) statistical characteristics of the received signals.

Each consideration is discussed with regard to motivating MFP.

Much of the literature has concentrated on a range independent representation of the sound field. In the range independent environment the critical parameters vary by a negligible about for a fixed range. Computations for long ranges required that range of interest be subdivided into smaller segments. This method offered a more manageable approach until recent developments in numerical methods for solving the wave equation given the boundary conditions significantly vary over long ranges. We approach MFP with no requirement to subdivide the search range of interest. This approach provides good flexibility to compute the propagation field in range independent environments. 


\subsection{Conventional Source Position Estimators}

\subsubsection{The Bartlett Estimator}

We begin to define the linear cross correlator or Bartlett estimator by considering the generalized case for the conventional beamformer [29]. We now assume that the complex ocean is characterized a solution to the wave equation that is

$$
w(\theta)=x\left(\theta_{s}\right)=\alpha e\left(r_{s}, z_{s}\right),
$$

where $E\left\{|\alpha|^{2}\right\}=\sigma_{s}^{2}$ models the strength of a random source at some range and depth.

The average noise power $\sigma_{\mathrm{n}}^{2}$, is also included as a part of the total measurement model. The vector $\mathbf{e}(\mathrm{r}, \mathrm{z})$ is the replica field which describes the underlying structure of the signal.

The array output power is given by equation (2.13) and the output of the conventional or Bartlett MFP is estimated by

$$
\begin{aligned}
\hat{P}_{B a r l}(\hat{\theta}) & \equiv w^{H}(\hat{\theta})\left\{\frac{1}{N} \sum_{k=1}^{N} x_{k}\left(\theta_{s}\right) x_{k}^{H}\left(\theta_{s}\right)\right\} w(\hat{\theta}) \\
& =w^{H} \hat{\boldsymbol{R}} w,
\end{aligned}
$$

where $\hat{\boldsymbol{R}}$ is the estimated covariance matrix; averaged over N independent measurements of the array output. Equation (2.15) attains a maximum value when $\boldsymbol{w}(\hat{\theta})=\boldsymbol{x}\left(\theta_{s}\right)$, indicating the main impetus into the use of matched field processors. Equation (2.15) suggests that we construct a large number of trial solutions. The best estimate of the 
source position is given by the maximum value of the power as $\theta$ is varied over the selected search domain. A three dimensional plot of $\hat{P}_{\text {Bar }}$ is called an ambiguity surface, it reflects the estimated power of the source for specified range and depth points in the search space. The quality of the estimator is then judged by the width of the peak and sidelobe levels which depend on the particular conditions.

\subsubsection{The Maximum Likelihood Estimator}

The "maximum likelihood" (ML) estimator, first introduced by Capon et. al., is popular because of its ability to suppress undesirable sidelobes that degrade the ability to estimate the source location [31]. It is a nonlinear estimator in that the inverse of $\mathbf{R}$ is required and that its resolving capability increases with SNR. Equivalent to beamforming with arrays, we want to minimize the contributions for undesired directions while maintaining the gain as constant along a particular look direction. This is equivalent to solving the condition

$$
\min _{w} \boldsymbol{w}^{\boldsymbol{H}} \boldsymbol{R} \boldsymbol{w} \text { subject to } \quad\left|\boldsymbol{w}^{\boldsymbol{H}} \boldsymbol{e}(r, z)\right|=1 .
$$

The solution to this optimization problem, assuming positive definite covariance matrices, is given by the weight vector

$$
w_{M L}=\frac{R^{-1} e(r, z)}{\boldsymbol{e}^{H}(r, z) R^{-1} e(r, z)}
$$

The weights of this beamformer are such that the spatial spectrum is a maximumlikelihood estimate of the power being received as a function of wavenumber (angle) if the noise-only covariance matrix is known [32]. The constraint in equation (2.16) fixes 
the gain of the steering vector or in the case of MFP the replica field, the direction of look $\mathbf{w}$ to be unity. The degree of suppression depends on the angular separation between the direction of the signal to the be suppressed, and the current look direction as well as the power level of the signal, and the array geometry. All this information is incorporated into equation (2.14), which is solved for the weight vector that is used in equation (2.14) to estimate the actual power. Forcing the energy to be a minimum thereby reduces the contributions from the wavefronts arriving from other directions and the noise field. Substituting equation (2.16) into equation (2.14) the Maximum Likelihood (ML) beamformer is defined as

$$
P_{M L M}(r, z)=\frac{1}{\left[e^{H}(r, z) R^{-1} e(r, z)\right]} .
$$

This procedure is referred to as the "maximum likelihood method" (MLM) because it resembles the form of the estimator to determine the ML estimate of a sine wave of known frequency in white Gaussian noise. When the covariance matrix is known the MLM yields the optimal array gain [32]. If it is not known, as is usually the case, we use its maximum likelihood estimate as defined in equation (2.17).

The maximum likelihood approach is studied because it provides greater sidelobe suppression within the ambiguity surface. The degree of suppression depends on the angular separation between the direction of the signal to be suppressed, the power level of the signal and array geometry. The MLM offers superior resolving power as compared to the Bartlett. This is shown by starting with $I=R^{1 / 2} R^{-1 / 2}$ which gives 


$$
\left[e^{H} R^{1 / 2}\right]\left[R^{-1 / 2} e\right]=1
$$

The Cauchy-Schwarz inequality is applied to obtain

$$
\left[e^{H} R e\right]\left[e^{H} R^{-1} e\right] \geq 1
$$

which gives

$$
P_{B a n}(r, z) \geq P_{M L M}(r, z)
$$

It can be seen from the matrix expression in the denominator of equation (2.17) that the determination of the value of the maximum likelihood function involves calculating products of functions. If the modeled field and measured field correlate closely with each other for every individual hydrophone, the denominator of equation (2.17) will be small and hence, $\mathrm{P}_{\mathrm{MLM}}(\mathrm{r}, \mathrm{z})$ will be large. The results of the MLM are displayed in an identical fashion as the output data of the previously described Bartlett estimator. 


\section{CHAPTER 3 \\ ACOUSTIC MODELING OF OCEAN ENVIRONMENTS}

\subsection{Overview}

This chapter describes sound propagation modeling in realistic ocean environments. Since a key feature of matched field processing is to incorporate the physics of the environment into the signal processing scheme, we account for characteristics of this complex media. Conventional beamformers assume infinite, and homogeneous media in equilibrium; this assumption is not applicable in shallow water signal processing problems. We provide a full description of the ocean model used in this investigation. Earlier acoustic propagation models were based on ray tracing techniques to model the local direction and intensity of a point source in a homogeneous medium [33], [34]. Variations in sound propagation which emanate from a point source, are a function of both time and space, are properly handled by these models. Ray theory accounts for complex effects of inhomogeneities in the water, multipath effects, and roughness from the top and bottom boundaries. The energy is viewed to travel along multiple ray paths having different travel times. The drawbacks of ray theory are that it requires each point of the sound front be traced out along its path of propagation and it does not accurately represent low frequency sources. Here, we apply modern wave theory starting with the general three-dimensional wave equation. Wave theory provides the basis for the analysis of wave propagation in layered complicated media [32],[33]. We pose our problem as a boundary value problem with initial conditions describing the location and boundaries on which the wave propagates. This allow the formulation of two 
mathematical methods that are applied to approximate the reduced wave equation or Helmholtz equation.

Next, we describe the method of normal modes which is an efficient for solving sound propagation problems in horizontally-stratified environments or waveguides. This method is applied to range dependent problems. Range dependence requires the division the full range into a number of segments, each with different range independent properties. The acoustic parameters (i.e. density, the sound speed, and attenuation) do not depend on the range within each segment.

Lastly, we derive and motivate the Parabolic Equation (PE) method as a method for efficient numerical computation of the replica data to be used in the signal processing algorithms. The PE and method of normal modes are contrasted. A description of the software which implements the PE is provided.

\subsection{Ocean Acoustical Model}

The ocean environment is an extremely complex medium and no single numerical method of modeling is capable of capturing most of its acoustic characteristics. However, in recent years, numerous developments in acoustic modeling incorporate additional environmental effects allowing for a more realistic treatment of three dimensional propagation and reverberation. We choose an inhomogeneous layered ocean in which to conduct MFP. Figure 3.1 depicts a realistic ocean environmental model along with its canonical modeling parameters. This model is frequently used by the research community and has proven to be an excellent research tool. It models shallow water characteristics more realistically that other models. 
sea surface,time varing roughness

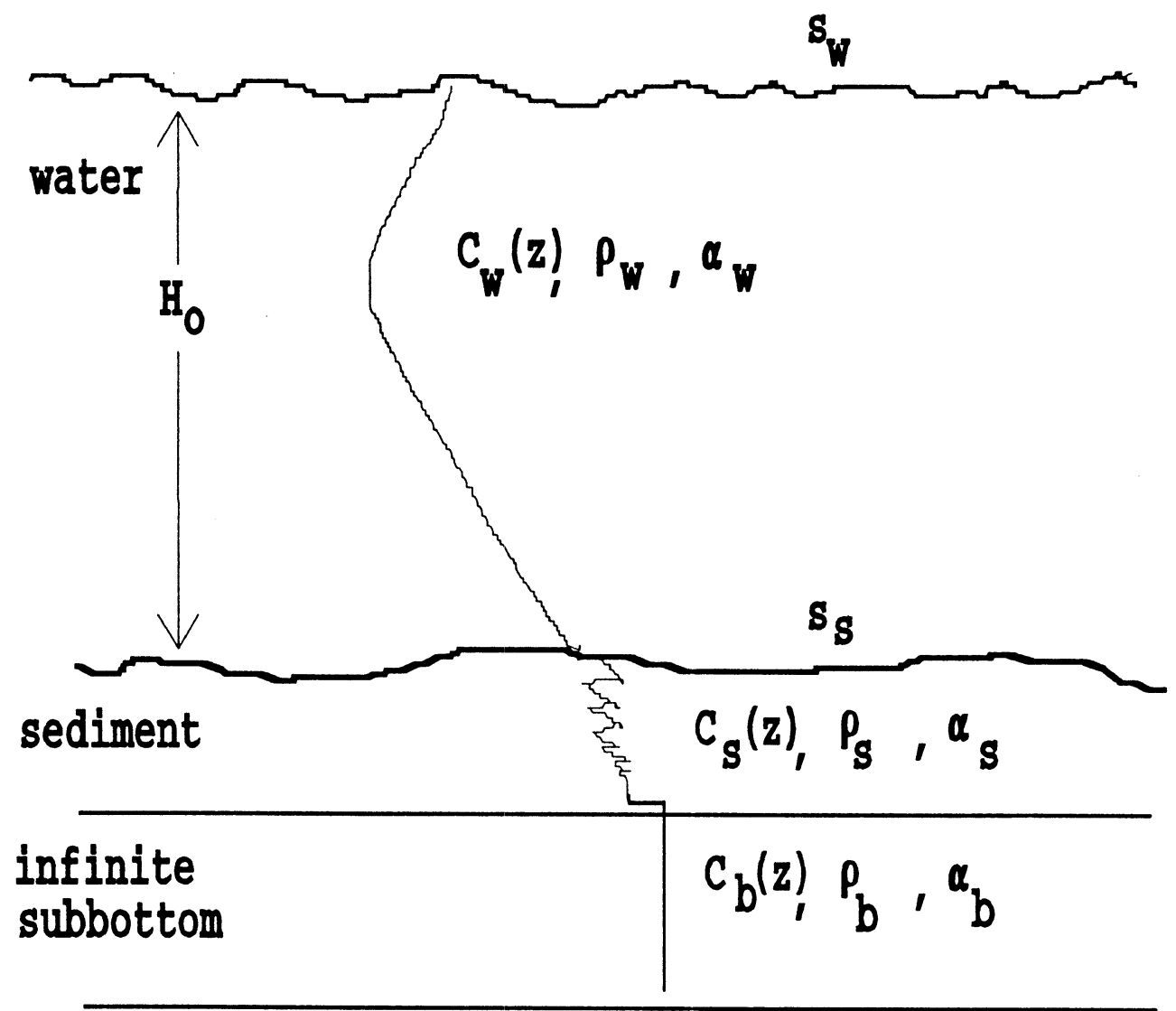

Figure 3.1 Ocean Environment Model 
The ocean is modeled as a half space divided into three layers: a water column of depth $\mathrm{H}_{\mathrm{o}}$, a sediment or interface layer, and a semi-infinite subbottom. In the water column the sound speed in $c_{w}(z)$ is allowed to vary deterministically with depth, while density $\rho_{\mathrm{w}}$ and volume attenuation $\alpha_{\mathrm{w}}$ are taken to be constant over depth and range dependent. The sediment layer is treated in exactly the same way: and arbitrary sound speed profile $c_{s}(z)$, a constant density $\rho_{s}$, and a constant volume attenuation $\alpha_{s}$. The subbottom, is treated as a solid with depth-independent properties: $c_{b}(z)$ is the shear speed, $\rho_{b}$ the density and $\alpha_{\mathrm{b}}$ the compressional attenuation. The ocean surface (pressure release) and ocean floor are treated as rough boundaries with rms roughness heights given by $s_{w}$ and $S_{\mathrm{s}}$ respectively.

The critical quantity which characterizes the complex pressure field is the variation of sound speed velocity $c$ in water. The speed of sound depends on the density, $\rho$ and the bulk modulus of elasticity, $\beta$, of the material. Hence, the wave velocity is given by

$$
c=\sqrt{\beta / \rho},
$$

where the bulk modulus of elasticity, $\beta$, varies with temperature, pressure, and salinity and the density, $\rho$, depends on the chemical composition of the water. Variations in the sound speed velocity in the ocean are relatively small. Thus, small changes $c$ significantly affect the propagation of sound in the ocean. Typically $c$ ranges between 1440 meters/second and 1560 meters/second. Here the sound is parameterized to vary 
in the first two layers. Losses due to sheer and compressional attenuation in the bottom layer and volume are included.

We consider the shallow water ocean where the deep sound channel does not exist. Shallow water propagation is defined by the ratio of the water depth $\mathrm{H}_{\mathrm{o}}$ to the acoustic wavelength. The ocean is accepted as shallow water when this ratio is not large compared to unity. The energy is then considered to undergo scattering and absorption at the boundaries. The reflection on the bottom produces attenuation of the sound waves, and significant losses of acoustic energy occurs over long distances.

This model now allows us to investigate match field localization problems which will typically fall into the two dimensional category. In addition it handles long range propagation.

\subsection{Wave Theory}

A mathematical discussion for the acoustic pressure of a point source near the water surface is presented. The acoustic field will be characterized by the scaler velocity potential $\Phi(\mathrm{x}, \mathrm{y}, \mathrm{z}, \mathrm{t})$, the acoustic particle velocity which is the negative gradient of $\Phi$, and the acoustic pressure $\rho \partial \Phi / \partial \mathrm{t}$. We consider a source, where it is assumed to have harmonic time dependence with frequency $\omega$ radians per second we write

$$
\Phi(r, z, t)=p(r, z) e^{-j \omega t}
$$

The general inhomogeneous wave equation which describes sound wave propagation in water is: 


$$
\nabla^{2} \Phi+k^{2} p=-4 \pi A \delta\left(z-z_{o}\right) e^{j \omega t}
$$

together with the proper boundary conditions at the upper and lower interfaces, at a point source, and very large distances away from the source. In Cartesian coordinates, equation (3.3) takes the form

$$
\frac{\partial^{2} p}{\partial x^{2}}+\frac{\partial^{2} p}{\partial y^{2}}+\frac{\partial p^{2}}{\partial z^{2}}+k^{2} p=-4 \pi A \delta\left(z-z_{o}\right) e^{j \omega t}
$$

where

$$
\begin{aligned}
& p=p(x, y, z), \text { the wave field in Cartesian coordinates; } \\
& k=2 \pi f / c(x, y, z), \text { the wavenumber; } \\
& f=\text { source frequency, } \\
& c=c(x, y, z), \text { the sound speed profile; and, } \\
& z, z_{o}=\text { point source located at } z=z_{0} .
\end{aligned}
$$

The wave equation can also be formulated in a cylindrical coordinate system. The cylindrical convention allows inclusion of the radial spreading experienced by the source. In the cylindrical coordinate system, the vertical $\mathrm{z}$ axis through the point source and the horizontal range $\mathrm{r}$ measured with the $\mathrm{z}$ axis. The Laplacian operator is in cylindrical coordinates is

$$
\nabla^{2}=\frac{\partial^{2}}{\partial r^{2}}+r^{-1} \frac{\partial}{\partial r}+\frac{\partial^{2}}{\partial z^{2}}
$$

The wave equation for the acoustic pressure $p$ in cylindrical coordinates $r$ and $z$ is 


$$
\begin{gathered}
\frac{\partial^{2} p}{\partial r^{2}}+\frac{1}{r} \frac{\partial p}{\partial r}+\frac{\partial^{2} p}{\partial z^{2}}- \\
\frac{1}{\rho(z)} \frac{d \rho}{d z} \frac{\partial p}{\partial z}+\frac{1}{r^{2}} \frac{\partial p}{\partial \theta^{2}}+k^{2}(z) p=-\frac{1}{2 \pi r} \delta\left(z-z_{s}\right) \delta(r)
\end{gathered}
$$

with the boundary conditions

$$
p(r, 0)=0, \text { and }\left[\frac{\partial p}{\partial z}\right]_{z=h}=0
$$

Equation (3.7) models the pressure release boundary conditions, $p(r, 0)=0$, and a no flow boundary condition at a constant depth. The right hand side of equation assumes a source in the cylindrical coordinate system. The local wavenumber is defined by

$$
k(z)=\frac{\omega}{c(z)}
$$

The fourth term of equation (3.6) drops due to the second boundary condition in equation 3.7. The $\theta$-dependent term drops out since contributions in the horizontal or azimuthal direction are often negligible. Equation (3.5) is now rewritten as a two-dimensional linear differential equation with sound speed and density depending only on depth $z$ :

$$
\begin{aligned}
\frac{1}{r} \frac{\partial p}{\partial r}\left(r \frac{\partial p}{\partial r}\right] & +\rho(z) \frac{\partial}{\partial z}\left(\frac{1}{\rho(z)} \frac{\partial p}{\partial z}\right)+\frac{\omega^{2}}{c^{2}(z)} p+k^{2}(z) p \\
& =-\frac{1}{2 \pi r} \delta\left(z-z_{s}\right) \delta(r)
\end{aligned}
$$

Equation (3.9) is the well known Helmholz equation. The solution to equation (3.9) describes the acoustic far field in the ocean, caused by a single frequency continuous 
wave point source at $\left(r=r_{o}, z=z_{o}\right)$. We begin with the normal mode solution which requires solving the depth dependent problem which is precisely the mathematical in equation (3.8).

\subsubsection{Normal-Mode Solutions to the Wave Equation}

We apply normal mode theory to matched field localization for long range propagation problems. It is restricted to propagation problems with little bottom interaction and very weak range dependence of the environment. Low-frequency sound propagation in shallow water can be accurately treated by normal-mode theory, which arises from consideration of the Helmholtz equation for the acoustic vector potential that is due to a unit source of harmonic frequency $\omega$ located at the depth axis at $(r, z)$ [34]. This theory has proven to be a valuable tool for the prediction of propagation loss. It is an exact solution to the wave equation for assumed environmental conditions and not limited by many of the assumptions required for ray theory models. The normal mode solution is a technique for computation of acoustic propagation loss in a horizontally stratified ocean. In summary it is an eigenvalue problem which requires standard iteration procedures to determine the roots of a characteristic equation. Thus, the problem resolves itself into obtaining initial estimates for the roots.

The first theoretical and experimental investigations of low frequency sound propagation in shallow water was made by Pekeris [9]. The general theory of many layered problems is discussed by Tolstoy. Tolstoy's work provides the theoretical background to obtain normal mode solutions due to a simple point source in a waveguide [35]. Normal mode descriptions are accurate and relative simplistic. The field at any 
point in the waveguide is immediately and directly accessible through normal modes. For range-independent waveguides, the sound speed, density, and absorption are functions of depth $z$, only and the wave equation is solved accordingly.

We begin by separating equation (3.8) into $r$ - and $z$ - parts without regard to any particular source, but with full consideration for boundary conditions. Using the separation of variables, we formulate a solution to the Helmholtz equation of the form $\mathrm{p}(\mathrm{r}, \mathrm{z})=\mathrm{R}(\mathrm{r}) \mathrm{Z}(\mathrm{z}) . \quad$ Equation (3.8) now becomes

$$
\frac{1}{r} \frac{\partial p}{\partial r}\left(r \frac{\partial p}{\partial r}\right]+\rho(z) \frac{\partial}{\partial z}\left(\frac{1}{\rho(z)} \frac{\partial p}{\partial z}\right)+\frac{\omega^{2}}{c^{2}(z)} p+k^{2}(z) p=0
$$

This equation can be satisfied by setting each component equal to a constant. The separation constant $k^{2}$ is used to obtain the modal equation:

$$
\begin{aligned}
\rho(z) \frac{d}{d z}\left[\frac{1}{\rho(z)} \frac{d Z(z)}{d z}\right]+\left[\frac{\omega^{2}}{c^{2}(z)}-k^{2}\right] Z(z) & =0, \\
Z(0) & =0, \\
\frac{d Z}{d z}(D) & =0 .
\end{aligned}
$$

Equation (3.10) is a classical Sturm-Liouville eigenvalue problem whose properties are well known [36]. We assume that the functions in equation (3.10) are real and that at $\mathrm{Z}_{\mathrm{m}}(\mathrm{z})$ it is continuously differentiable.

This modal equation has an infinite number of solutions. They are characterized by a shape function $\mathrm{Z}_{\mathrm{m}}(\mathrm{z})$ and a horizontal propagation constant $\mathrm{k}_{\mathrm{m}}$. The function $\mathrm{Z}_{\mathrm{m}}(\mathrm{z})$ is an eigenfunction and $\mathrm{k}_{\mathrm{m}}{ }^{2}$ is an eigenvalue. Each combination of an eigenvalue and 
eigenfunction represents a normal mode of the whole pattern. An additional property of the Sturm-Louville problem is that the modes are orthogonal. That is,

$$
\int^{h} \Psi_{k} \Psi_{m}(z) d z=0, \quad l \neq m,
$$

In order to simplify the results, we normalize the modal functions so that

$$
\int_{0}^{D} \frac{Z_{m}^{2}(z)}{\rho(z)} d z=1 .
$$

We can now write the pressure as a sum of normal modes:

$$
p(r, z)=\sum_{m=1}^{\infty} R_{m}(r) Z_{m}(z) .
$$

Equation (3.14) is substituted into equation (3.9) and we obtain:

$$
\begin{aligned}
& \sum_{m=1}^{\infty}\left\{\frac{\rho(z)}{r} \frac{\partial}{\partial r}\left[\frac{r}{\rho(z)} \frac{\partial R_{m}(r)}{\partial r}\right) Z_{m}(z)\right\}+ \\
& \sum_{m=1}^{\infty}\left\{R_{m}(r)\left[\rho(z) \frac{d}{d z}\left[\frac{1}{\rho(z)} \frac{d Z(z)}{d z}\right]+\frac{\omega^{2}}{c^{2}(z)} Z_{m}(z)\right]\right\}=\frac{-\delta(r) \delta\left(z-z_{s}\right)}{2 \pi r} .
\end{aligned}
$$

This implies,

$$
\sum_{m=1}^{\infty}\left[\frac{1}{r} \frac{\partial}{\partial r}\left[r \frac{\partial R_{m}(r)}{\partial r}\right] Z_{m}(z)+k_{m}^{2} R_{m}(r) Z_{m}(z)\right]=\frac{-\delta(r) \delta\left(z-z_{s}\right)}{2 \pi r}
$$

Next we apply the operator: 


$$
\int_{0}^{D}(\cdot) \frac{Z_{m}(z)}{\rho}(z) d z
$$

to equation (3.15), and recognize that because of the orthogonality property only the $n$th term in the sum remains. This gives

$$
\frac{1}{r} \frac{\partial}{\partial r}\left[r \frac{\partial R_{m}(r)}{\partial r}\right]+k_{m}^{2} R_{m}(r)=\frac{-\delta(r) Z_{m}\left(z_{s}\right)}{2 \pi r \rho\left(z_{s}\right)}
$$

To obtain the solution we assert the condition $\mathrm{kr} \gg 1$, which implies the horizontal ranges of interest must exceed the wavelength of interest. Then the solution to equation (3.18) requires the Hankel function of the first kind and is given as

$$
R_{m}(r)=\frac{i}{2 \rho\left(z_{x}\right)} Z_{m}\left(z_{s}\right) H_{0}^{1}\left(k_{m} r\right)
$$

We put all this together and the solution to equation (3.9) can be represented as a sum of eigenfunctions or normal modes as:

$$
p(r, z)=\sum A_{m} Z_{m}\left(z_{s}\right) Z_{m}(z) H_{o}^{(1)}\left(k_{m} r\right)
$$

The set $\left\{A_{m}\right\}$ are the coefficients of the normal modes. The solution set is chosen by requiring outgoing waves at very large $r$, which requires the Hankel function of the first kind. This function has the asymptotic form:

$$
v(r)=H_{o}^{(1)}(k r) \equiv J_{o}(k r)+i N_{o}(k r)
$$

which represents an outgoing cylindrical wave: 


$$
v(r) \sim(2 \pi)^{1 / 2}(k r)^{-1 / 2} \exp \left\{i\left(k r-\omega t-\frac{\pi}{4}\right)\right\} .
$$

The factor $r^{-1 / 2}$ shows cylindrical spreading. The intensity is proportional to $v^{2}$ and falls off as $1 / r$.

We complete the mathematical modeling description with transmission loss (TL). The TL defined as:

$$
T L(r, z)=-10 \log \left|\frac{p(r, z)}{p^{o}(r=1)}\right|^{2},
$$

where the denominator denotes the pressure for the source in free space. Transmission loss is the ratio (expressed in decibels) of the acoustic energy transmitted at range $r$ relative to energy transmitted at one yard.

These modes are then fitted to a prescribed source, and finally the specific acoustic field is formulated as a combined sum. Equation (3.20) represents the set of discrete normal modes. The full field is composed of both a continuous part and a discrete part, but it can be shown that the continuous spectrum generally provides negligible contributions beyond the nearfield of the source. We also note that each frequency component has a slightly different set of normal modes and wavenumbers. A limitation of the normal mode is determined the accuracy of the eigenvalues. Errors in the eigenvalues occur as phase shifts in the range dependence of the acoustic field. These errors can rapidly degrade the accuracy of the normal mode representation, especially as the distance from the source increases. An accurate range independent 
solutions to the Helmholtz equation offers better match field localization results. We derive a solution which overcomes the above limitation in the following section.

We have now formed a full solution often used to solve the wave equation at a single frequency which is the sum of normal modes each multiplied by the Hankel function $H_{o}^{(1)}\left(k_{m} r\right)$ which provides the range dependence. A comprehensive discussion of normal mode theory can be found in texts written by Clay et. al. and Tolstoy [34] and [35].

\subsubsection{Parabolic Equation Method}

We present one of several parabolic approximations to the reduced wave equation and its numerical solution to account for strong range dependent problems. Here the acoustic parameters (i.e. the sound speed profile, density, and attenuation) depend on both depth and range. This is due to recent developments to incorporate additional environmental effects which describe the ocean. In many cases the narrow angle Finite Element Parabolic Equation (FEPE) method is not accurate enough to represent the wave field. The wide angle parabolic equation (PE) was first derived by Collins based upon a square root operator which is represented by a rational function approximation to handle the desired order of accuracy [37] and [38]. We employ this wide angle PE to simulate the replica fields in this investigation. In this chapter we discuss important assumptions to derive the wide angle PE. We include a description and listing of the software which implements the PE method.

The PE was introduced in association with electromagnetic waves by $M$. Leontovich and V.A. Fock in 1946 and adapted to underwater sound propagation by 
F.D. Tappert and R. H. Hardin in 1976 [39] and [40]. It is a computationally efficient procedure for computing field values for ranges up to approximately 10 kilometers. It can also handle range-independent sound speed profiles in underwater applications.

The major advantage of the PE is the availability of efficient numerical methods available to solve parabolic equations. Continued research of ocean fronts, bathymetry, and geoacoustics of the seabed has refined several other PE methods for modeling propagation at low frequency [41] and [42]. Numerical computations on high speed processing units has resulted in more sophisticated codes to model realistic environments in reasonable execution times. Another advantage of the PE approximation over the plane wave solution and the normal mode solution is its ability to handle energy loss due to absorption and transmission in the bottom.

The PE approximation to the reduced wave equation is derived by the use of a matrix that splits the total field into a transmitted field and a reflected field. The matrix that splits the wave equation is arbitrary, therefore different PEs exists. We derive the a PE approximation which handles wide angle propagation as opposed to strictly narrow angle propagation. The inhomogeneities are assumed to be slowly varying with range which offers greater flexibility over plane wave solutions. A major advantage of the PE over the Helmholtz equation is that it can be solved using a marching scheme, whereas equation (3.9) requires a simultaneous solution everywhere in space.

We start by considering equation (3.9). We consider the ocean boundaries behaving as a waveguide but the pressure due to the localized source has the behavior $\mathrm{p} \sim 1 / \mathrm{r}^{1 / 2}$ at long ranges. It is desirable to first remove the cylindrical spreading factor. 
We now define the field $u$ by $p=r^{1 / 2} u(r, z)$ and substitute $p$ into equation (3.9). The result is

$$
\frac{\partial^{2} u}{\partial r^{2}}=-\left(k^{2}+\frac{\partial^{2}}{\partial z^{2}}+\frac{1}{4 r^{2}}\right) u
$$

The term $1 / 4 \mathrm{r}^{2}$ is negligible at ranges beyond a few wavelengths and will be dropped. Equation (3.24) is rewritten as

$$
\frac{\partial^{2} u}{\partial r^{2}}=-\left(k^{2}+\frac{\partial^{2}}{\partial z^{2}}\right) u
$$

In each range-independent region , $u$ satisfies equation (3.25). Next we apply operator theory and define the operator

$$
Q^{2}=k^{2}+\frac{1}{k_{o}^{2}} \frac{\partial}{\partial z^{2}}
$$

The results are derived using a modified pressure $u=p / \alpha$, where $\alpha$ is a function of sound speed $c$ and density $\rho$. Specifically, a higher-order Padé approximation for the operator $\mathrm{Q}$ is used for a class of PE models capable of producing solutions to problems involving very wide angles of propagation as well as long range and depth variations in the sound speed conditions. The high-order outgoing PE result is

$$
\frac{\partial u}{\partial r}=i k_{o} \sum_{j=1}^{n} \frac{a_{j, n}\left[\rho \frac{\partial}{\partial \sigma} \frac{1}{\rho} \frac{\partial}{\partial \sigma}+K^{2}-k_{o}^{2}\right]}{k_{o}^{2}+b_{j, n}\left[\rho \frac{\partial}{\partial \sigma} \frac{1}{\rho} \frac{\partial}{\partial \sigma}+K^{2}-k_{o}^{2}\right]} p
$$

where $\mathrm{a}_{\mathrm{j}, \mathrm{n}}$ and $\mathrm{b}_{\mathrm{j}, \mathrm{n}}$ are the Padé coefficients. 
A detailed mathematical derivation of equation (3.27) is presented in Appendix A. Equation (3.27) was first derived by Collins and is classified as a more accurate and stable PE for range dependent problems.

The disadvantage of the parabolic equation method is its limitation to nearly radial propagation. Radial propagation occurs when large downward sloping bottoms appear near the source. In addition, the PE requires a starting field to initialize the solution. The PE produces transmission loss $(\mathrm{Db})$ verses range for a point sources located in range-dependent oceans.

\subsection{Acoustic Data Simulation Using The PE}

Computer codes which implement various PE algorithms exist at many institutions and research laboratories. A notable computer code is FEPE, developed at the Stennis Space Center by Dr. M.D. Collins. This PE code, hereafter referred to as the PE Solver, is used worldwide for both research and applications. This code which numerically solves the wave equation using the PE method is especially useful because it:

1) computes the complex acoustic pressure in range dependent environments;

2) computes the transmission lose for range dependent environments;

3) is accurate for propagation angles of nearly 90 degrees;

4) allows the ocean environment to be tailored;

5) allows for one of five mathematical approaches to define the initial field

6) can be used directly with high-level graphics software;

7) achieves desired accuracy with a small number of Padé coefficients; and 
8) is numerically efficient due to the uses of a finite-difference algorithm.

The PE method requires that the field be specified at some point or a starting solution. Several starting solutions can be specified including a normal mode solution. We choose the Green's starter which is accurate, easy to construct and recommended by Collins. The accuracy of the PE is determined by the number of Padé coefficients (1-5) specified. The code solves the parabolic equation model based on Galerkin's method, alternating directions and Crank-Nicolson integration. Basically, parabolic differential equations are setup to represent a realistic ocean environment. These equations are solved by specifying the field for all depths at an initial range and depth, and by finding the field at successive ranges and depths by using successive numerical solutions.

The environmental conditions, specification of range and depth computational intervals, and source frequency and source location are input parameters to PE Solver. Details for specifying the input parameters and description of the above features are provided in the FEPE User's Manual [43].

The computer code FEPE is written in FORTRAN IV and is portable between various digital computer workstations with minor modifications. In order to verify the baseline PE code, we tested it using several documented examples. Specifically, the transmission loss curves produced for a source in a range-independent, homogeneous waveguide were in close agreement with those of example 2 and example 3 of reference [43]. 
In this investigation, we wish to simulate the array data and replica fields in range dependent ocean environments. To this end, detailed software modifications were made to the PE Solver specifically for this research. These modifications

to the baseline PE solver software included:

1) specification of additional control parameters inputs;

2) computation of fields for both a source and jammer;

3) direct implementation of the Bartlett estimator;

4) direct implementation of the MLM estimator which requires inversion of a matrix; and,

5) off-line storage of vertical array data and replica field data for direct input into the GLRT algorithm.

Figure 3.3 depicts the logical flow of the modified PE solver code. The modifications includes the addition of new code as well changes to existing code. A complete listing of the software code performing the modified PE Solver calculations is provided in Appendix B. 


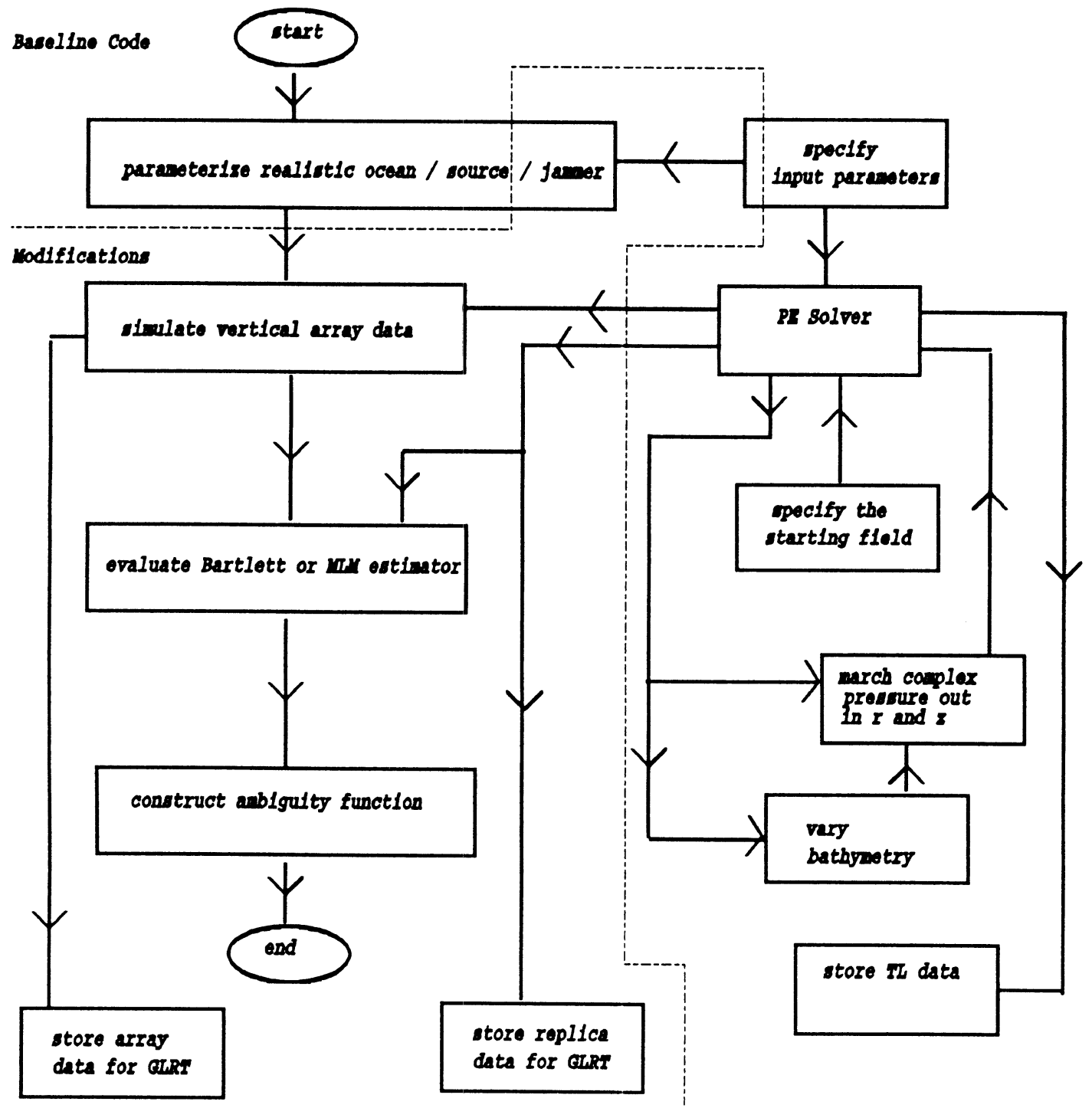

Figure 3.2 The Modified PE Solver 


\section{CHAPTER 4 \\ REDUCED-RANK SIGNAL PROCESSING APPLIED TO SOURCE LOCALIZATION}

\subsection{Introduction}

This chapter reformulates the MFP problem as a joint detection and estimation problem. The objectives are two fold: detection followed by source estimation which is dependent upon the ability to decide if a signal in the field of view has been detected. We propose a novel Generalized Likelihood Ratio Test (GLRT) solution which can be applied to a wide variety of MFP problems. The GLRT is a well known and useful approach to detect signals that include unknown parameters [44] and [45]. We apply the GLRT to solve matched field localization problems in high noise environments.

We begin with a brief background of historical developments. A short description of the theory of linear models is provided to establish that general array measurements can be handled naturally in conjunction with the GLRT. Next, we define the descriptions of the array measurements in terms of their components. The components are the signal, directional interference, and white noise formulated in terms of linear models. In this formulation, we emphasize the fact that strong directional interference has been incorporated into the matched field localization problem. In the following section the GLRT is derived. We present a systematic approach combined with some heuristics to jointly estimate a mean vector and a structured covariance matrix from data. To accomplish this, we make use of several key theorems and propositions from the multivariate statistical literature. The GLRT is implemented in the framework of reduced 
rank signal processing, and we develop it as a powerful approach for extracting information in noisy environments. Lastly, the GLRT is evaluated using prototype plane waves before proceeding to MFP applications in Chapter 5.

\subsection{Background}

Detecting a signal in the presence of unknown directional interference plus white Gaussian noise can be accomplished by means of adaptive detection algorithms such as one derived by Kelly [2]. The problem is formulated with two classes of data: the secondary data which is assumed to be free of signal components, and a primary class which may contain unknown complex signal scalars. A likelihood ratio decision rule is derived and its performance is evaluated. The output of the likelihood ratio algorithm is a decision of signal presence or absence, rather than a sequence of processed data from which interference has been removed.

Another detection method is the Principal Components Inverse (PCI) method [44]. The PCI method requires training data to estimate the interference. The training data is constrained to contain only strong interference components. Tufts investigated the PCI method for adaptive detection when the noise covariance matrix is approximately low rank, and only a limited amount of noise data is available [46]. Here, the signal component is within the training data, and it must be weaker that the low rank interference or it will be removed. The PCI method rapidly adaptively nulls the interference through application of the weight vector. It was shown that a likelihood ratio test statistic can be written as a function of the received data vector $\mathbf{x}$ and a set of filter weights [47]. The method of estimating the weight vector is called the Sample 
Matrix Inverse (SMI). Kirsteins extended the PCI methodology to improve the method for determining the weight vectors. It was demonstrated experimentally and theoretically for the near rank one case, that the PCI method weight vector estimate as opposed to the SMI weight vector estimate would give better higher output SNR [48].

Detection of a low rank signal in the presence of unknown strong low rank interference through the use of a reduced rank generalized ratio test is proposed by Kirsteins [48]. We generalize his algorithm to problems where the signal of interest is not completely known.

\subsection{Signal and Noise Models}

In this section we describe the signals measured on an array of hydrophones. The received waveforms contain a signal component originating from the source of interest and a low rank interference component due to random wavefronts or white noise.

It is assumed that the observed waveform consists of signal plus noise or noise alone. The received waveform at the array of sensors is represented by

$$
x^{T}(t)=\left[x_{1}(t), x_{2}(t), \ldots, x_{N}(t)\right]
$$

where $\mathrm{N}$ is the number of sensors in the array. Since we are reformulating the MFP problem as a joint detection and estimation problem this involves choosing between one of the following binary hypotheses for the received data and parameter estimator. The alternatives are to decide whether or not a signal $\mathbf{s}(t)$ is present and the hypothesis maybe written as: 


$$
H_{o}: \quad x(t)=n(t), \text { all } t \in(O, T),
$$

or

$$
H_{1}: \quad x(t)=s(t)+n(t), \text { all } t \in(O, T),
$$

where $\mathbf{n}(\mathrm{t})$ is the noise process at time $t$.

We model the signal, $\mathbf{s}$ as deterministic with unknown parameters. The noise, denoted as $\mathbf{z}$, is comprised as a low rank Gaussian component plus white Gaussian noise. The functional form for a $k$ th measurement vector in equations (4.2) and (4.3) then becomes

$$
H_{o}: \quad x_{k}=\sum_{m=1}^{r} a_{m}^{k} e_{m}+z_{k} \quad \text { (interference only ) }
$$

and

$$
H_{1}: \quad x_{k}=b_{k} s(\theta)+\sum_{m=1}^{r} a_{m}^{k} e_{m}+z_{k} \quad \text { ( signal plus interference) }
$$

$b_{\mathbf{k}}$ models an unknown gain and phase shift of the signal. $\mathbf{s}(\theta)$ represents the received signal due the source of interest. The strong "low rank" interference noise model for of the $k$ th array snapshot is: 


$$
n_{k}=\sum_{m=1}^{r} a_{m}^{k} e_{m}
$$

where $\left\{e_{m}\right\}$ are $n \times 1$ vectors that constituted the basis or modes which generate the interference. $\left\{a_{m}^{k}\right\}$ correspond to the amplitude of the $m$ th interference mode and modeled as zero mean complex Gaussian.

Reduced rank signal processing requires that we operate on data matrices, so we collect a sequence of independent vector measurements or snapshots to form the data matrix $\mathbf{X}$, organized as follows:

$$
X=\left[x_{1}\left|x_{2}\right| \ldots \mid x_{K}\right]
$$

It follows that the $\mathrm{K}$ snapshots of data in equation (4.5) are multivariate Gaussian and the objective is to determine if the data set $\left[x_{1}, x_{2}, \ldots, x_{K}\right]$ comes from $H_{o}$ or $H_{1}$.

Spatially localized noise maybe significant compared with other system noises and it does ultimately set limits on the performance of the receiving aperture and on any subsequent processing. The low rank model of equation (4.6) accounts for them. Sources of interference in a realistic ocean environment are biologics, rain, internal waves, wind noise, and surface traffic. Interference may also be intelligently produced (i.e. electronic countermeasures). Low rank modeling means that a time domain or spatial waveform can be modeled as a linear combination of $r$ linearly independent 
"modes", where $r$ is less than the number of sensors in the measurement array. We now formulate the "low rank" noise component of the entire data matrix $\mathbf{X}$ as:

$$
n_{k}=\sum_{m=1}^{r} a_{m}^{k} e_{m}=\left[e_{1} e_{2} \ldots e_{r}\right] a^{(k)}
$$

where $a^{(k)}=\left[a_{2}^{(k)} a_{2}^{(k)} \ldots a_{r}^{(k)}\right]^{T}$. The $k$ th column of $\mathbf{A}$ is $\mathbf{a}^{(k)}$.

Low rank signal and noise models impose structure on data measurements that can be exploited to solve interference cancellation problems and signal detection problems. First, the singular value decomposition of $\boldsymbol{A E}$, can be directly subtracted from full rank $\mathbf{X}$ yielding:

$$
\begin{aligned}
\boldsymbol{X}-\boldsymbol{A} \boldsymbol{E} & =\sum_{i=1}^{N} \lambda_{i} u_{i} v_{i}^{H}-\sum_{i=1}^{r} \lambda_{i} u_{i} v_{i}^{H} \\
& =\sum_{i=r+1}^{N} \lambda_{i} u_{i} v_{i}^{H}
\end{aligned}
$$

which removes much of the low rank interference from the data [46]. Second, we utilize the covariance structure resulting from the contribution of Expression (4.6). The covariance matrix $\mathbf{R}$ is the sum of two covariance matrices $\left(\boldsymbol{R}=\boldsymbol{Q}+\sigma^{2} \boldsymbol{I}\right)$ where $\mathbf{Q}$ is the covariance matrix of zero-mean narrowband Gaussian noise. The eigenvalue decomposition of $\mathbf{R}$ is critical a priori knowledge and is given as: 


$$
\begin{aligned}
\boldsymbol{R} & =\boldsymbol{Q}+\sigma^{2} \boldsymbol{I}=\boldsymbol{U} \boldsymbol{\Lambda} \boldsymbol{U}^{\boldsymbol{H}}+\sigma^{2} \boldsymbol{I} \\
& =\sum_{i=1}^{r}\left(\lambda_{i}+\sigma^{2}\right) \boldsymbol{u}_{i} \boldsymbol{u}_{i}^{H}+\sigma^{2} \sum_{i=r+1}^{N} \boldsymbol{u}_{i} \boldsymbol{u}_{i}^{H} .
\end{aligned}
$$

Interference is strongly low rank when $\lambda_{k}>>\sigma^{2}$, where $\lambda_{k}$ is the $k$ th eigenvalue of the covariance matrix $\mathbf{R}$. $\mathbf{Q}$ is the covariance matrix of the vector in equation (4.6) and has rank $r$.

Under the noise only conditions we risk the possibility of handling an illconditioned covariance matrix. Although the above description may suggest that this method is limited to strong jammers in weak Gaussian noise, we will show that it can be successfully applied to suppress weak jammers in high noise environments.

We have made use of the concept that detecting a source in the presence of strong directional interference plus white noise can be formulated naturally in terms of linear models. This is indicated through the use of equations (4.4) and (4.5). Linear models provide the mathematical framework to draw inferences, test, and confidence regions about the estimated parameters [49]. We also point out that normal mode methods (Section 3.3.1) for prediction of underwater sound characteristics are a class of linear signal models.

\subsection{Derivation of The Generalized Likelihood Ratio Detector}

We derive a generalized likelihood ratio test statistic which detects a low-rank deterministic and unknown signal in white Gaussian noise. Our approach to the problem offers the following features:

(1) The covariance matrix $\mathbf{R}$ is unknown, but its eigenstructure is partially known. 
(2) The low rank noise structure is exploited.

(3) Signal free training data is not needed.

(4) An approximately Constant False Alarm Rate (CFAR) detector is derived.

The GLRT is based on standard likelihood ratio test (LRT) for choosing between hypothesis $H_{0}$ and $H_{1}$. This approach requires the maximization of two multivariate probability functions over the unknown parameters. We exploit our limited a priori knowledge as much as possible. Since the unknown parameters are replaced by their maximum likelihood (ML) estimates, the resultant test is known as the generalized likelihood ratio test [43]. We develop a signal processing algorithm in this section based on rules for reducing the rank of signal models and use it as a novel approach to matched field processing [50]. Specifically, we motivate and design a generalized likelihood ratio test (GLRT) which is a useful approach to detection of signals that include unknown parameters. The GLRT uses the probability density function of the received signal conditioned on the signal(s) with unknown signal scalers jointly with an unknown covariance matrix in the likelihood ratio test. The test consists of forming the likelihood ratio

$$
L(x) \equiv \frac{P\left(x / H_{o}\right)}{P\left(x / H_{1}\right)}{\stackrel{H_{1}}{H_{0}}}_{H_{0}}
$$

and attempting to maximize the numerator and denominator over the unknown parameters to obtain the GLRT. The decision is to choose $\mathrm{H}_{\mathrm{o}}$ if $\mathrm{L}<\eta$, or choose $\mathrm{H}_{1}$ if $\mathrm{L}>\eta$. We start with the null hypothesis $\mathrm{H}_{\mathrm{o}}$, for which the likelihood function is: 


$$
\prod_{k=1}^{K} f\left(\boldsymbol{R} \mid \boldsymbol{x}_{k}\right)=\frac{1}{\sqrt{(2 \pi)^{K}|\boldsymbol{R}|^{K}}} \exp \left\{(-1 / 2)\left[\sum_{k=1}^{K}\left(\boldsymbol{x}_{k}^{H} \boldsymbol{R}^{-1} x_{k}\right)\right]\right\}
$$

Similarly for the alternate hypothesis $\mathrm{H}_{1}$, the likelihood function is:

$$
\begin{aligned}
& \prod_{k=1}^{K} f\left(\boldsymbol{R}, \boldsymbol{b}_{k} \mid s x_{k}\right) \\
= & \frac{1}{\sqrt{(2 \pi)^{K}|\boldsymbol{R}|^{K}}} \exp \left\{(-1 / 2)\left[\sum_{k=1}^{K}\left(\left(x_{k}-b_{k} s\right)^{H} R^{-1}\left(x_{k}-b_{k} s\right)\right)\right]\right\} .
\end{aligned}
$$

The likelihood function in equation (4.12) is a function of $\mathbf{R}$ and the likelihood function in equation (4.13) is a function of $\mathbf{R}$ and $\mathbf{b}$. These quantities are emphasized to be variables and not parameters by denoting them as $\{\hat{b}\}$ and $\hat{\boldsymbol{R}}$. They are the optimum estimators of $\mathbf{b}$ and $\mathbf{R}$ which are the scalars $\left\{\hat{b}_{L S}\right\}$ and the positive definite matrix $\hat{\boldsymbol{R}}$ that do the best job in maximizing the log of equations (4.12) and (4.13) since insufficient information is available for maximum likelihood estimation. However, we follow the rules of maximum likelihood estimation to obtain the above estimates in order to formulate the prescribed GLRT.

We apply the matrix identity 


$$
v^{H} R v=\operatorname{trace}(R V) \quad \text { where } V=v v^{H}
$$

to rewrite the quadratic expressions in equations (4.12) and (4.13). The test statistic is derived by substituting equations (4.12) and (4.13) into equation (4.11). Ignoring the constants, it can be rewritten as:

$$
z(\theta)>_{H_{\odot}}^{H_{H_{1}}} \frac{\max _{R, b, \theta} \frac{1}{|\boldsymbol{R}|^{N / 2}} \exp \left(-\frac{N}{2} \operatorname{trace}\left(R^{-1} S_{1}\right)\right)}{\max _{R, \theta} \frac{1}{|R|^{N / 2}} \exp \left(-\frac{N}{2} \operatorname{trace}\left(\boldsymbol{R}^{-1} S_{0}\right)\right)}
$$

where $S_{o}=\frac{1}{K} X X^{H}, S_{1}=\frac{1}{K}\left(X-s b^{H}\right)\left(X-s b^{H}\right)^{H}$, and $\mathbf{R}, \mathbf{b}$, and source parameters $\theta=(\mathrm{r}, \mathrm{z})$ are unknowns. Evaluating $\mathbf{z}(\theta)$ requires that the unknowns $\mathbf{R}$ and $\mathbf{b}$ be replaced by their optimum estimates.

Under both $\mathrm{H}_{\mathrm{o}}$ and $\mathrm{H}_{1}$, computing the GLRT requires the estimation of $\hat{\boldsymbol{R}}$. We can construct the estimated covariance matrix $\hat{R}$ by exploiting theorem 11.3.1 in Anderson [52]. The optimum estimate of $\mathrm{R}$ given its partially known eigenstructure is:

$$
\hat{R}=\sum_{k=1}^{r} \hat{\lambda}_{k} \hat{\boldsymbol{v}}_{k} \hat{\boldsymbol{v}}_{k}^{H}+\hat{\sigma}^{2} \sum_{k=r+1}^{N} \hat{\boldsymbol{v}} \hat{v}^{H}
$$

where the $\left\{\hat{\lambda}_{k}\right\}$ and $\left\{\hat{v}_{k}\right\}$ are the eigenvalues and corresponding eigenvectors of $\mathbf{S}_{0}$, and 


$$
\hat{\sigma}^{2}=\frac{1}{N-r} \sum_{k=r+1}^{N} \hat{\lambda}_{k}
$$

It follows from equation (4.14) that $\operatorname{trace}\left(\hat{\boldsymbol{R}}^{-1} S\right)=N$. We now have the $\mathrm{H}_{\mathrm{o}}$ likelihood function

$$
f\left(x_{k} / \hat{\boldsymbol{R}}\right)=\frac{1}{(2 \pi)^{N}|\hat{\boldsymbol{R}}|^{K / 2}} \exp \left(\frac{-K N}{2}\right)
$$

Ignoring the constants in equation (4.18) then

$$
f\left(x_{k} / \hat{\boldsymbol{R}}\right) \propto \frac{1}{|\hat{\boldsymbol{R}}|^{K / 2}}
$$

Solve for $\max \left\{\frac{1}{|\hat{R}|^{K / 2}}\right\}$ which is equivalent to $\min \{|\hat{R}|\}$ or by definition of matrix

determinants:

$$
\hat{\lambda}_{1} \hat{\lambda}_{2} \ldots \hat{\lambda}_{r}\left(\sum_{k=r+1}^{N} \hat{\lambda}_{k}\right)^{N-r}
$$

where $\left\{\hat{\lambda}_{k}\right\}$ are the eigenvalues values of $S_{o}$.

Next we wish to calculate the optimum value of the $\mathrm{H}_{1}$ likelihood function. Under $\mathrm{H}_{1}$, attempting to maximize the log likelihood function requires the joint estimation of $\hat{\boldsymbol{b}}$ and $\hat{\boldsymbol{R}}$. Under $\mathrm{H}_{1}$, the optimum estimate of $\mathbf{R}$ is 


$$
\hat{\boldsymbol{R}}=\sum_{k=1}^{r} \hat{\lambda}_{k} \hat{\boldsymbol{u}}_{k} \hat{\boldsymbol{u}}_{k}^{H}+\hat{\sigma}^{2} \sum_{k=r+1}^{N} \hat{\boldsymbol{u}}^{\boldsymbol{H}}
$$

where

$$
\hat{U} \hat{\Lambda} \hat{U}^{H}=P^{\perp} S_{o} P^{\perp}
$$

and

$$
\hat{\sigma}^{2}=\frac{1}{N-r} \sum_{k=r+1}^{N} \hat{\lambda}_{k}
$$

Similarly, we now have the $\mathrm{H}_{1}$ likelihood function

$$
f\left(x_{k} / \hat{b}_{1}, \hat{b}_{2}, \ldots, \hat{b}_{k}, \hat{R}\right)=\frac{1}{(2 \pi)^{K}|\hat{R}|^{K / 2}} \exp \left(\frac{-K N}{2}\right)
$$

We claim that the solution the ordinary least-squares estimate of $\left\{\mathbf{b}_{\mathrm{LS}}\right\}$ is

$$
\hat{b}_{L S}=\frac{X^{H} S}{s^{T}}
$$

Equation (4.22) is also the solution to the well known least squares problem $\min \left\|\boldsymbol{X}-\boldsymbol{s} \boldsymbol{b}^{\boldsymbol{H}}\right\|_{F}^{2}$. The operator \|\|$_{F}^{2}$ denotes the squared Frobenius norm. Proof of the claim is accomplished by showing that any other solution must always increase the eigenvalues of $S_{1}$, therefore increasing the result of equation (4.22). Let $b_{0}$ be some arbitrary solution to the least squares problem, then 


$$
\begin{aligned}
x-s b_{o}^{H} & =X-s\left(\hat{b}_{L s}-b_{o}\right)^{H}+s \hat{b}_{L S}^{H} \\
& =X-\frac{s s^{H} X}{s^{H} s}+s\left(\hat{b}_{L S}-b_{o}\right)^{H} \\
& =P_{\perp} X+s\left(\hat{b}_{L S}-b_{o}\right)^{H}
\end{aligned}
$$

where $P^{\perp}=I+\frac{s s^{H}}{s^{H} S}$.

We note that

$$
\boldsymbol{P}_{\perp}\left(\boldsymbol{P}_{\perp} \boldsymbol{X}+s\left(\hat{b}_{L S}-b_{o}\right)^{H}\right)=\boldsymbol{P}_{\perp} \boldsymbol{X}
$$

We now apply theorem 4.5 from Stewart to equation (4.27) to get

$$
\begin{aligned}
\mathrm{T}_{k} & \leq \sigma_{k}\left\|P_{\perp}\right\|_{2}, \quad k=1,2, \ldots, N \\
& \leq \sigma_{k}, \quad k=1,2, \ldots, N
\end{aligned}
$$

where $\mathrm{T}_{k}$ and $\sigma_{\mathbf{k}}$ are the singular values of $\boldsymbol{P}_{\perp} \boldsymbol{X}$ and $\boldsymbol{P}_{\perp} \boldsymbol{X}+\boldsymbol{s}\left(\hat{b}_{L S}-b_{o}\right)^{H}$ respectively. The expression in equation (4.28) must be a strict inequality for some singular values since $\left\|\boldsymbol{P}_{\perp} \boldsymbol{X}\right\|<\left\|\boldsymbol{P}_{\perp} \boldsymbol{X}+\boldsymbol{s}\left(\hat{b}_{L S}-b_{o}\right)^{H}\right\|_{F}^{2} \quad$ if $\quad \hat{b}_{L S}-b_{o} \neq \mathbf{0}$. This is a direct application of theorem 4.5 from Stewart and thus we conclude that the ordinary least squares estimate of $\mathbf{b}$ is given by equation (4.25) [51].

To find the MLE of $\mathbf{R}$, we replace $\mathbf{b}_{\mathbf{k}}$ by its ordinary least squares estimate: 


$$
\hat{\boldsymbol{R}}=\frac{1}{K} \sum_{k=1}^{K}\left(x_{k}-\hat{b}_{k} s\right)\left(x_{k}-\hat{b}_{k} s\right)^{H}
$$

Substituting $\hat{b}_{L S}$ into equation (4.29) we get

$$
\hat{R}=\frac{1}{K} \sum_{k=1}^{K}\left[x_{k}-s\left(\frac{x^{H} s}{s^{H} s}\right)^{H}\right]\left[x_{k}-s\left(\frac{x^{H} s}{s^{H} s}\right)^{H}\right]^{H}
$$

which reduces to

$$
\begin{gathered}
\left(I-\frac{s s^{H}}{s^{H} S}\right) \frac{1}{K} X X^{H}\left(I-\frac{s s^{H}}{s^{H} s}\right) \\
=P^{\perp} S_{o} P^{\perp} .
\end{gathered}
$$

This is a good result: It tells us to estimate the signal mean and remove it from the data before covariance matrix estimation. Thus proceeding in a similar manner as under $\mathrm{H}_{\mathrm{o}}$ to obtain $\mathrm{H}_{1}$, equation (4.21) reduces to:

$$
\hat{\lambda}_{1} \hat{\lambda}_{2} \ldots \hat{\lambda}_{r}\left(\sum_{k=r+1}^{N} \hat{\lambda}_{k}\right)^{N-r}
$$

where $\left\{\hat{\lambda}_{k}\right\}$ are the eigenvalues values of $\mathrm{P}^{\perp} \mathbf{S}_{0} \mathbf{P}^{\perp}, \mathbf{P}^{\perp}$ is the projection matrix $\left[I+\frac{s S^{H}}{S^{H} S}\right]$ which estimates the mean required for an optimum estimate of $\mathbf{R}$. 
The above results allow us to construct the likelihood ratio test. We substitute equations (4.20) and (4.29) into equation (4.11) and the GLRT is formulated as:

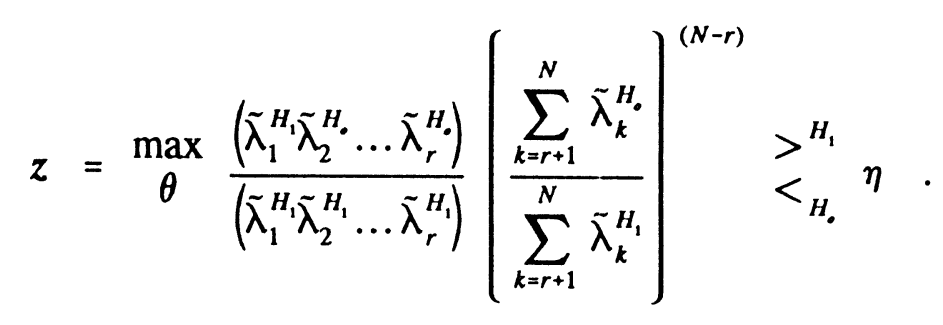

Equation (4.33) is a plausible result since implementation involves computing eigendecompositions of sample covariance matrices $\mathbf{S}_{\mathrm{o}}$ and $\mathbf{S}_{1}$. We emphasize that this method does not utilize prior knowledge about the functional form of the interference. It does utilize information which can be obtained from the data to approximate the rank of the interference.

\subsection{GLRT Performance Evaluation In An Infinite, Homogeneous Medium}

\subsubsection{Problem Formulation}

This section describes the evaluation of the GLRT given plane wave energy propagation in an infinite, homogeneous medium. The focal part of this experiment provides insight regarding the capability of the GLRT to detect the presence of a source. We begin with a description of the known and unknown parameters.

We impose structure on $\mathbf{R}$ by assuming the noise has a rank $r$ Gaussian component. The jammer or interference described in Section 4.3 is modeled as low rank and complex Gaussian. We do not have knowledge of its location nor its power. In 
addition we do not know the white Gaussian noise power. The signal of interest has rank one.

The experiments account for varying the source power, jammer power, and white noise power while the source position remains fixed at array boresight. The power of the jammer is fixed at $0 \mathrm{~dB}$ and its position relative to the source is allowed to change. We proceed to evaluate the GLRT's performance as a DOA estimator and describe simulation results.

We employ standard $N x l$ array steering vectors that model the source and jammer respectively and $\mathbf{n}$ models the measurement noise which is assumed to be Gaussian. The sensor spacing $d$, is selected as $\lambda / 2$ to avoid spatial alaising.

\subsubsection{Typical GLRT Outputs}

We conduct this experiment modeling two different length arrays to investigate the GLRT output changes due to the array null-to-null beamwidth. We compute null-to-null beamwidth using the standard formula:

$$
2 \sin ^{-1}(\lambda / N d)
$$

The shorter length array of eight sensors provides a null-to-null beamwidth of 28.95 degrees and the longer array of sixteen sensors provides a null-to-null beamwidth of 14.36 degrees. We average 20 array snapshots to estimate sample covariance matrices $\mathbf{S}_{\mathrm{o}}$ and $\mathbf{S}_{1}$. The averaging provides a processing gain of $13.01 \mathrm{~dB}$ against the white Gaussian noise. This experiment has many parameters which can be varied from experiment to experiment, so we judiciously choose a subset of parameters to perform 
MFP in one dimension with the GLRT. The particular simulation parameters are listed below each plot.

Plots in Figures (4.1) and (4.2) display the GLRT output verses DOA in degrees for a strong signal and weak signal relative to the jammer power respectively. 


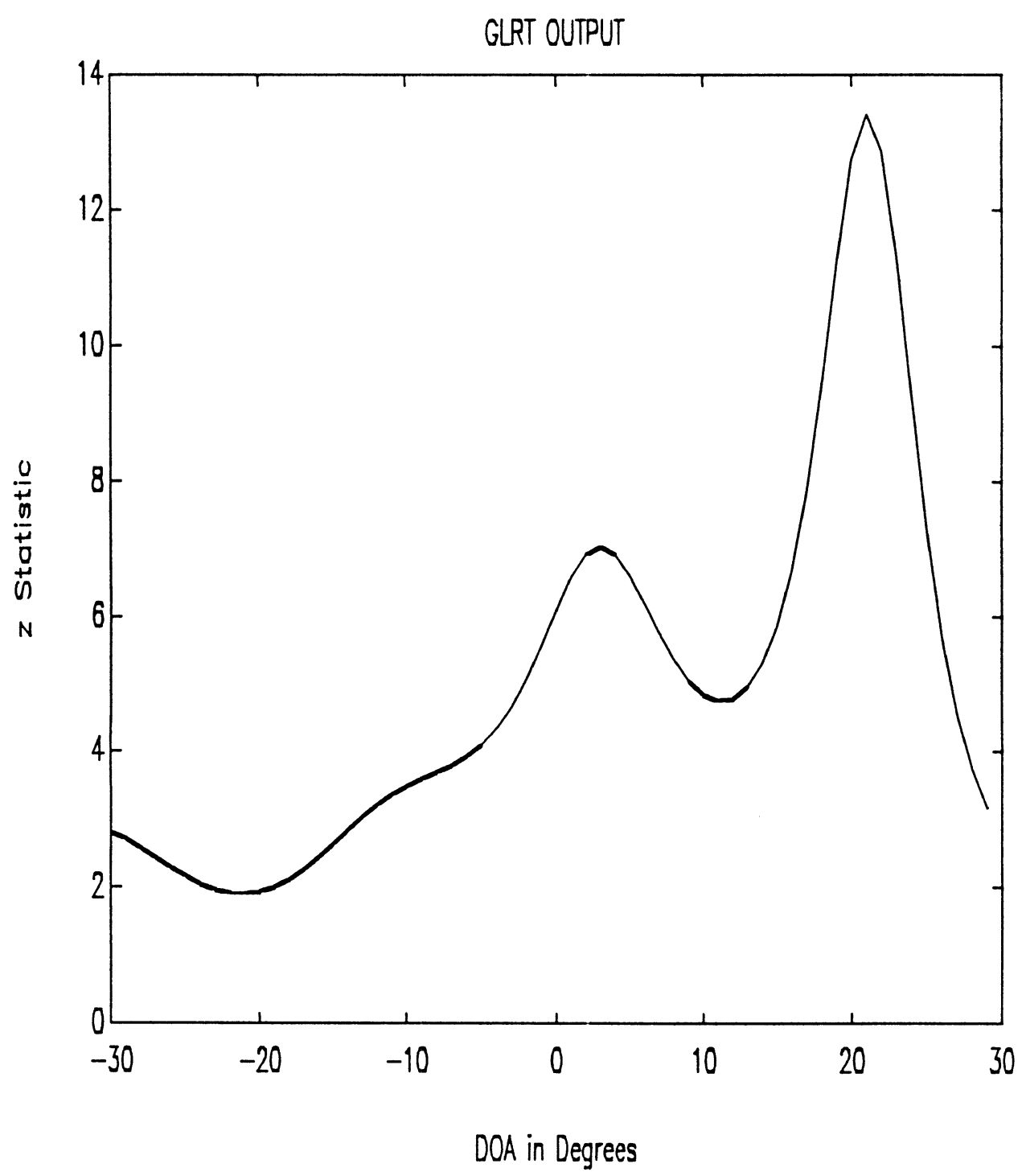

Figure 4.1 DOA Case 1: Weak source distantly space from a strong jammer.

The simulation parameters are: $\quad$ Number of sensors: 8

Null-to-null beamwidth: 28.95 degrees

Source power:

$-3.46 \mathrm{~dB}$

Jammer power and DOA: $0 \mathrm{~dB}$ and 20 degrees

White noise power:

$-5.53 \mathrm{~dB}$ 


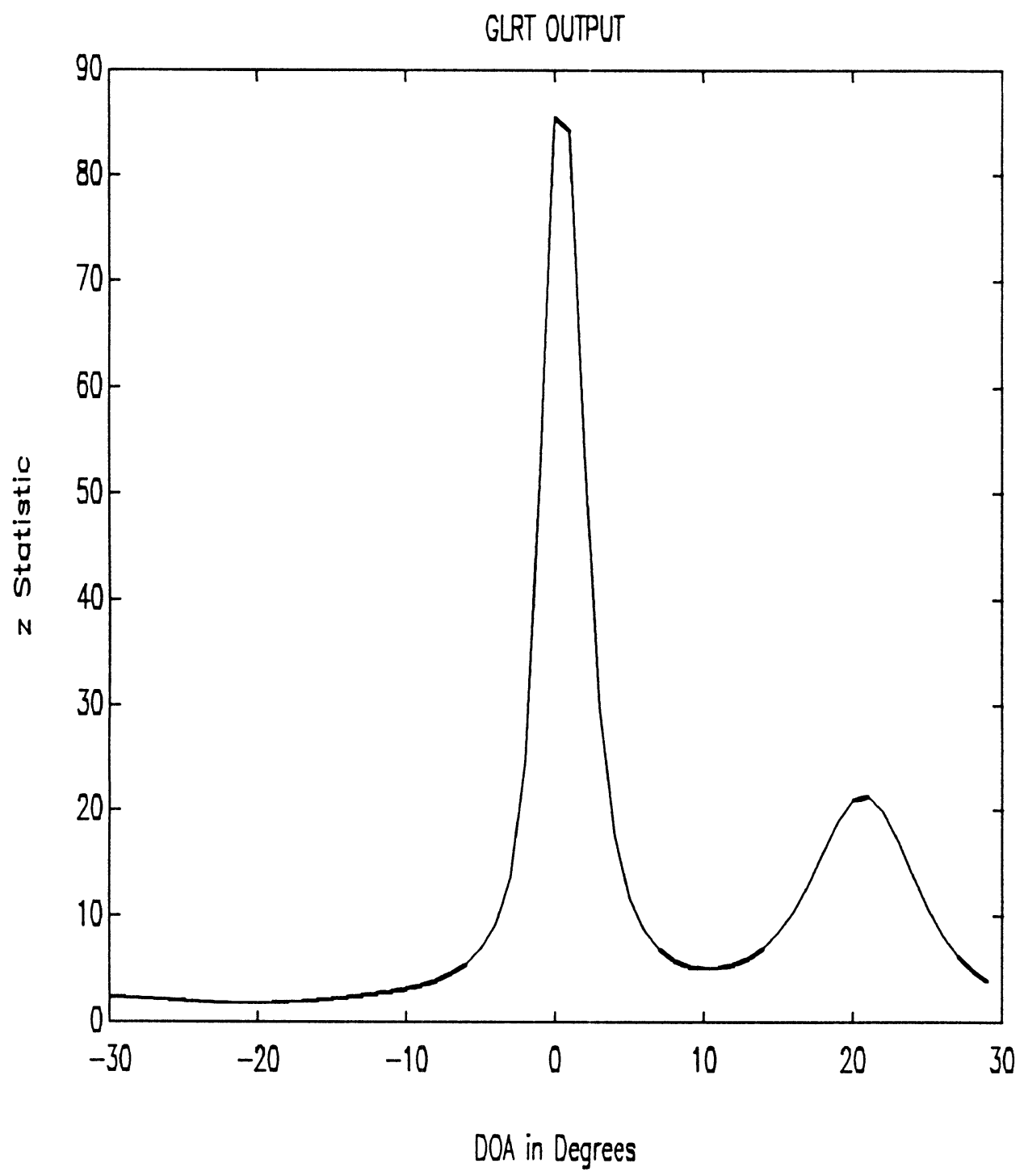

Figure 4.2 DOA Case 2: Strong source distantly spaced from a weak jammer.

The simulation parameters are: $\quad$ Number of sensors: 8

Null-to-Null Beamwidth: 28.95 degrees

Source power: $\quad-7.53 \mathrm{~dB}$

Jammer power and DOA: $\quad 0 \mathrm{~dB}$ and 20 degrees

White noise power: $\quad-5.53 \mathrm{~dB}$ 
We observe that the GLRT has no difficulty in distinguishing the source and jammer. This occurs irrespective of the source and jammer powers as long as there is reasonable separation between the source and jammer. We repeat the above simulations using the same test parameters. Here the jammer is moved closer 12 degrees closer to the source. and we observe that the GLRT continues to perform well. Figures 4.3 displays the result of this scenario. Next we remove the signal component from the initial model and decrease the power of the jammer by $2 \mathrm{~dB}$. Figure 4.4 demonstrates the GLRT's ability to detect a weak jammer given the correct assumption that the signal of interest is absent. 


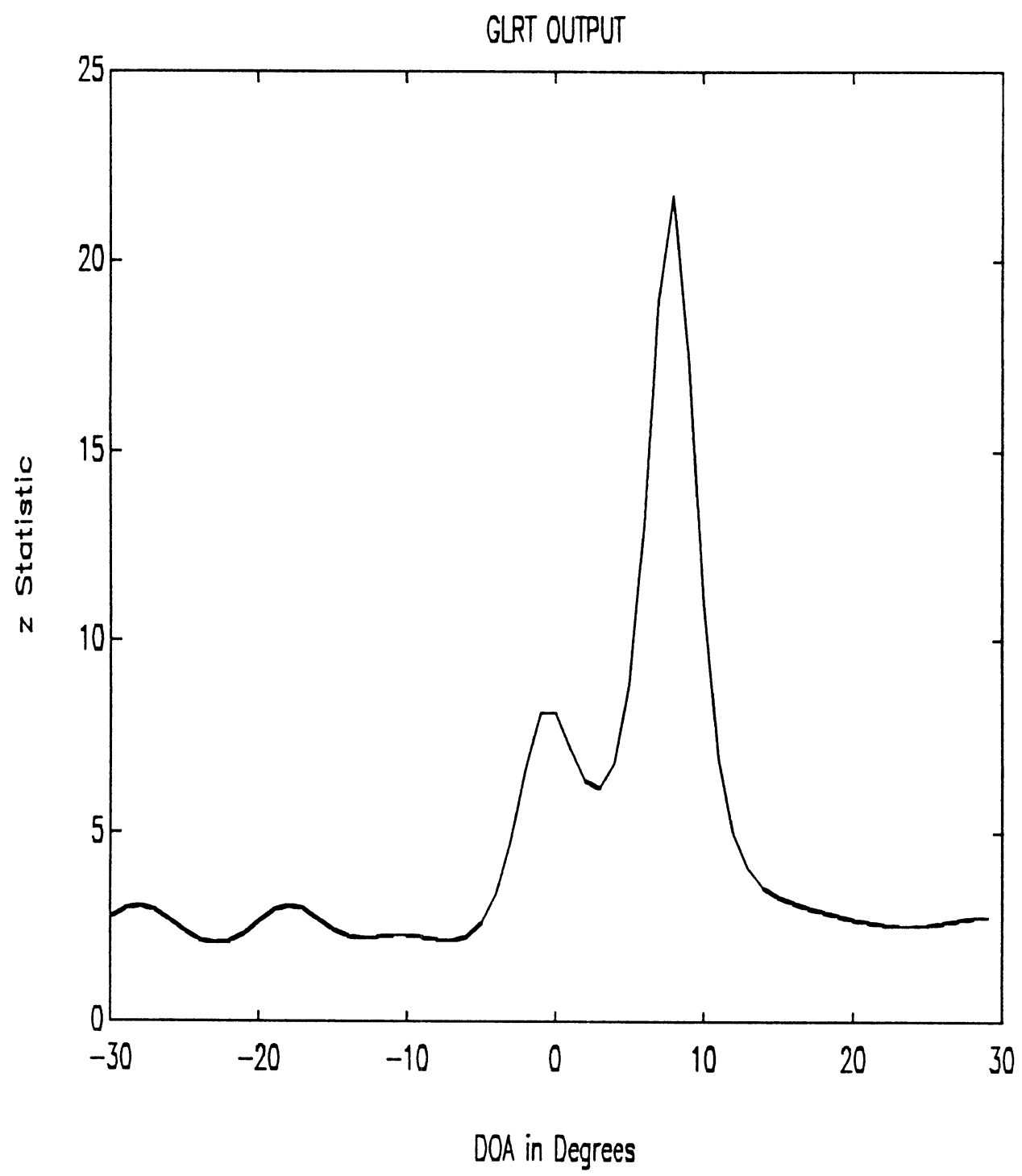

Figure 4.3 DOA Case 3: Weak source closely spaced to a strong jammer.

The simulation parameters are: Number of sensors: $\quad 16$

Null-to-Null Beamwidth: 14.36 degrees

Source power $-3.56 \mathrm{~dB}$

Jammer power and DOA: $0 \mathrm{~dB}$ and 8 degrees

White noise power: $\quad-5.53 \mathrm{~dB}$ 


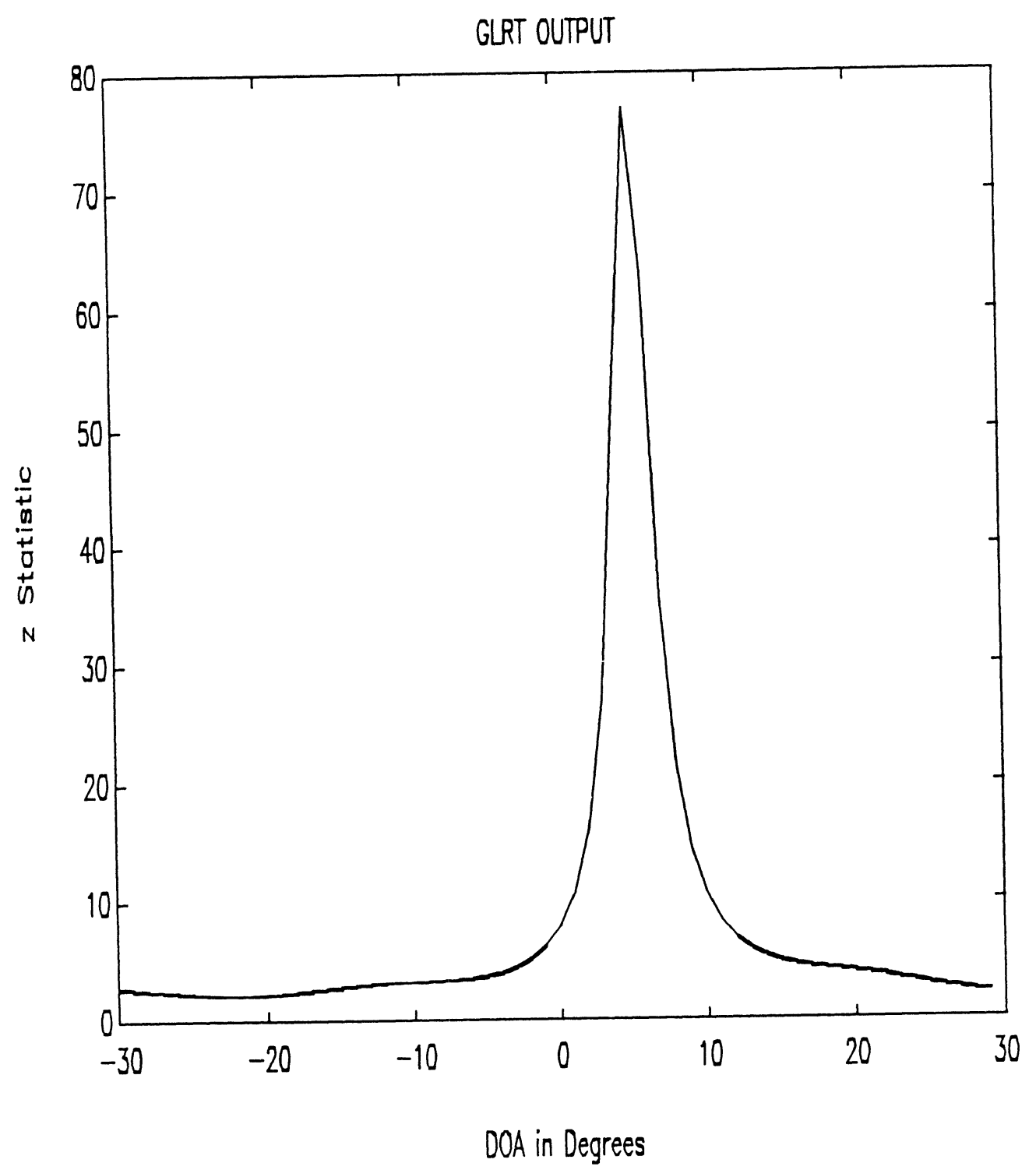

Figure 4.4 DOA Case 4: $\mathrm{H}_{\mathrm{O}}$ Assumption - Detection of a weak jammer.

The simulation parameters are:

Number of sensors:

Null-to-Null Beamwidth:

Source power:

Jammer power and DOA: $0 \mathrm{db}$ and 8 degrees

White noise power:
16

14.26 degrees

$7.56 \mathrm{~dB}$

$-5.53 \mathrm{~dB}$ 
The above plots for detecting the source in the presence of a jammer suggest that the GLRT does perform well under varied conditions. As we would expect, the ability to detect the source increases as we increase the signal power and increase the array aperture. Figures (4.5) and (4.6) show that the GLRT can reliably distinguish between the source and jammer as we move it closer to the source, increase its power, or decrease the array aperture. We push the GLRT to its limits by conducting simulations with the jammer at 5 degrees relative to the source. The signal and jammer powers are $-5.0 \mathrm{~dB}$ and $1 \mathrm{~dB}$ respectively. In Figure (4.5) the array aperture is small and the GLRT output for the source in masked by the proximity and power of the jammer. However, in Figure (4.6) given the identical systems parameters with a larger aperture a sourcejammer distinction becomes apparent. Note that the background noise is lowered from $5.53 \mathrm{~dB}$ to $-13.1 \mathrm{~dB}$ to aid the GLRT in distinguishing the source and jammer. 


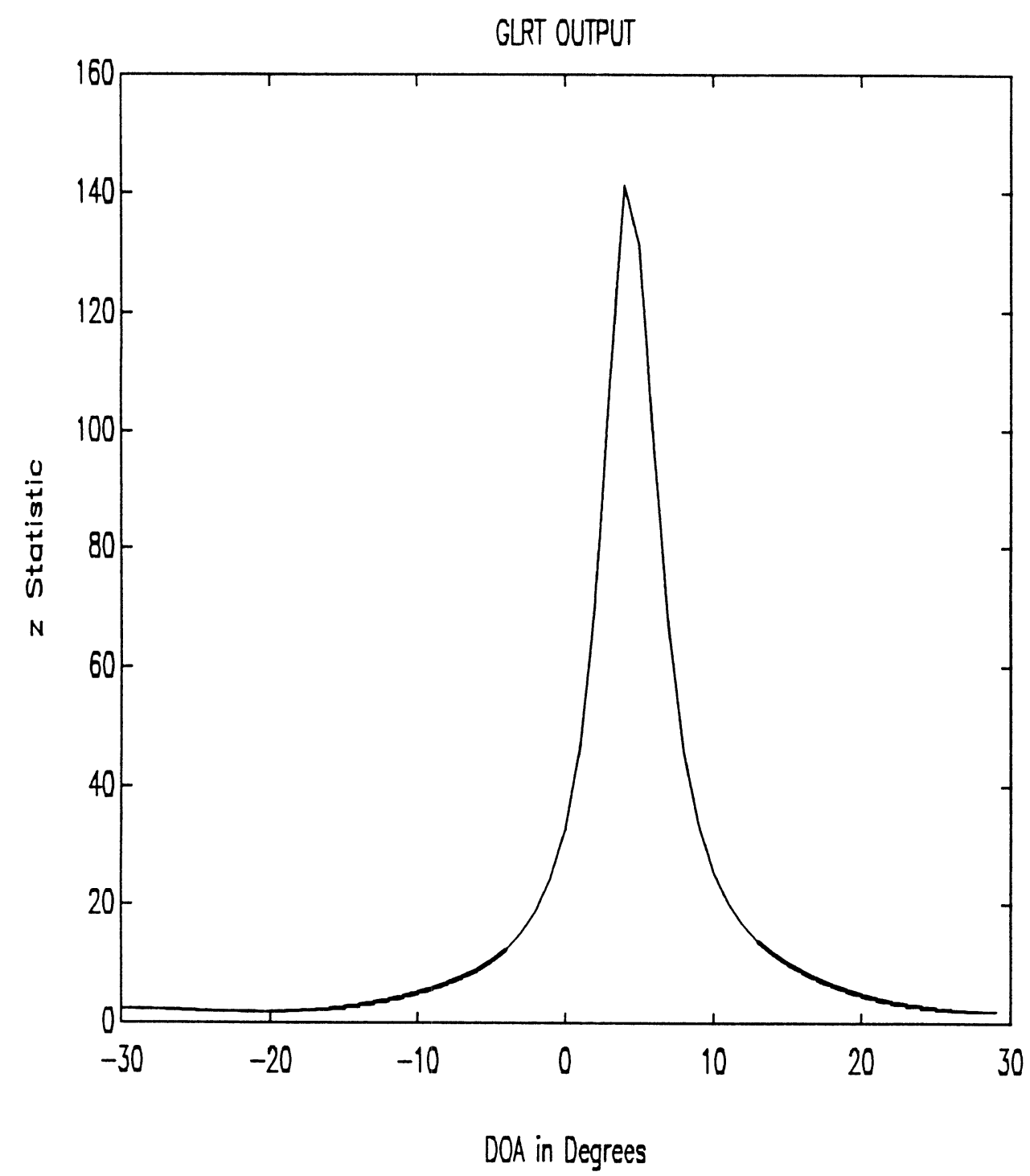

Figure 4.5 DOA Case 5: Weak source very closely spaced to a strong jammer. The simulation parameters are: Number of sensors: 8 Null-to-Null Beamwidth: 28.95 degrees Source Power: $-5.0 \mathrm{~dB}$ Jammer power and DOA: $0 \mathrm{~dB}$ and 5 degrees White noise power: $-13.1 \mathrm{~dB}$ 


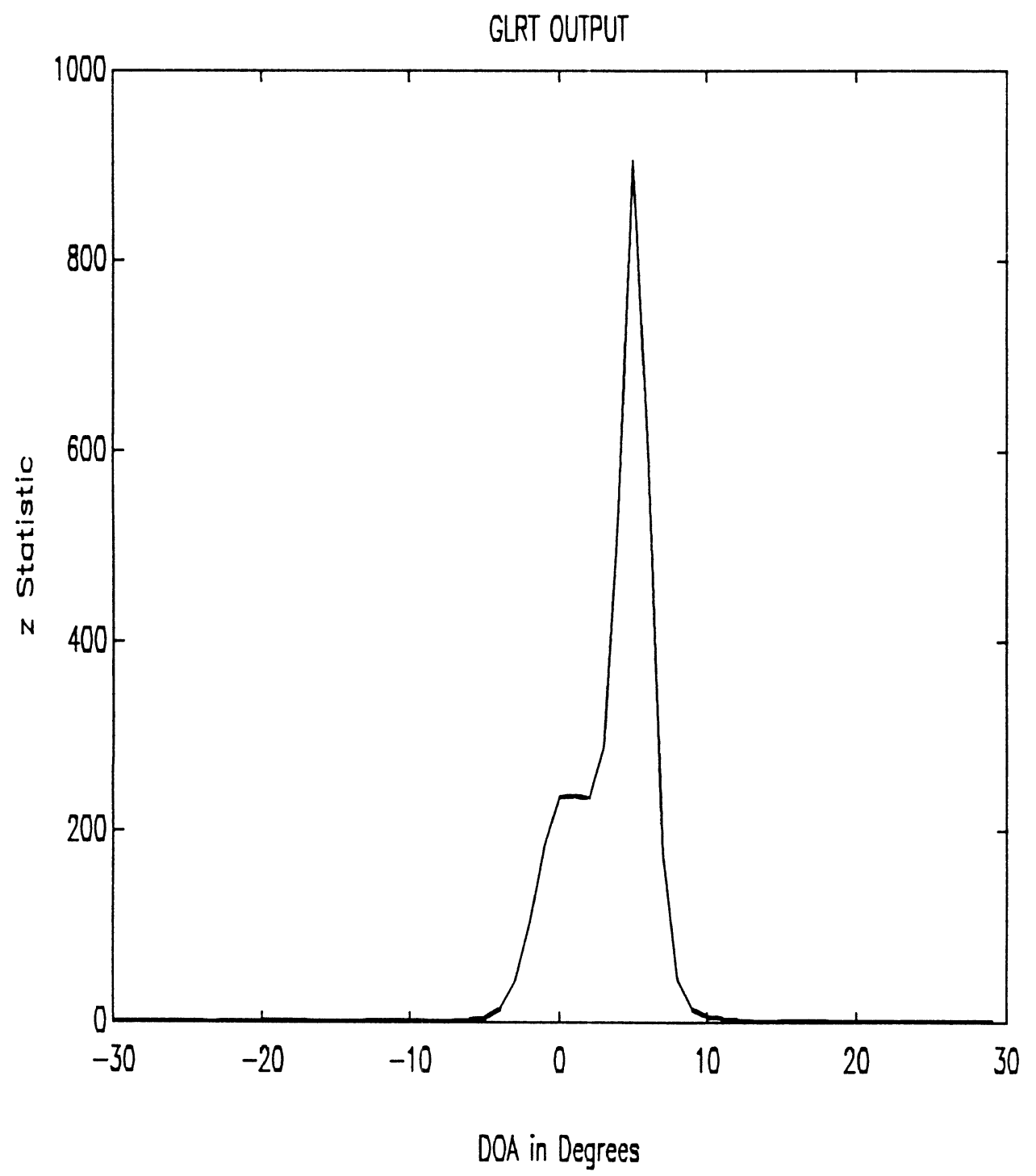

Figure 4.6 DOA Case 6: Weak source very closely spaced to a strong jammer.

The simulation parameters are: Number of sensors:

Null-to-Null Beamwidth: 14.36 degrees

SIR:

$-5.0 \mathrm{~dB}$

Jammer DOA:

5 degrees

White noise power:

$-13.1 \mathrm{~dB}$ 


\subsubsection{GLRT As A Detector}

This section presents experimental results to determine the performance of the GLRT. We have proposed the GLRT as a straight forward to derive and implement but not necessarily satisfying any optimality criterion. In the case where the threshold output of the generalized likelihood ratio is compared can be selected to achieve a constant false alarm rate (CFAR) performance. The complete algorithm provides a CFAR detector which is completely independent of the actual noise variance and the actual covariance matrix of the interference. In this work we examine the probability of detection $P_{D}$ verses the probability of false $P_{F A}$ alarm is computed with signal-to-jammer and jammer-to-noise as a parameters. We apply an optimal hypothesis testing procedure which does not require $a$ priori probabilities.

The likelihood function was derived in equations (4.10) and (4.11) completely characterizes the data assumed for each hypothesis and model. The sufficient statistic is computed for each observation vector and compared to the threshold $\eta$. We apply the Neyman-Pearson criterion which assumes that the false alarm-probability is constrained to be less than or equal to a specified value $\eta$. Simultaneously, we attempt to maximize the probability of detection. The following conventions are adopted:

1) $\quad P_{D}=\operatorname{Pr}[$ select $\mathrm{H} 1 / \mathrm{H} 1$ true] we say source present when it is, and

2) $\quad P_{\mathrm{FA}}=\operatorname{Pr}[$ select $\mathrm{H} 1 /$ Ho true $]$ we say source absent when it is not.

The above quantities are discussed through a parametric plot of $P_{D}$ verses $P_{F A}$. A complete description of hypothesis testing criteria can be found in [30]. 
The probability of detection equals the area under the noise-only density function to the right of the detection threshold setting. We approximate the noise only density by counting the number of occurrences in which the GLRT output statistic exceeds $\eta$ under $\mathrm{H}_{\mathrm{o}}$ and generating a histogram Hist $t_{\mathrm{o}}$ by selecting $\Delta \eta$ 's. Similarly, we approximate the signal-plus-noise density by counting the number of occurrences in which the GLRT output statistic exceeds $\eta$ under $\mathrm{H}_{1}$ and generating the histogram Hist, by selecting $\Delta \eta$ 's. The GLRT output is computed for 1000 trials and we repeat the above steps to approximate equations (4.32) and (4.33) which completely specify the receiver operating characteristics (ROC).

$$
\begin{gathered}
\hat{P}_{F A}=\frac{\text { No. of False Alarms }}{1000} \\
\hat{P}_{D}=\frac{\text { No. of Detections }}{1000}
\end{gathered}
$$

We specify several sets of parameters to vary between experiments to demonstrate the detection performance of the GLRT. The parameters demonstrate the influence of change the source power, the white noise power, the angle between the source and jammer and the array aperture. The ROC plots and the simulation parameters are presented in Figures (4.7) through (4.10).

These curves express the probability of detection verses the probability of false alarm with signal-to-noise ratio as a parameter. These results adhere to the properties 
of continuous likelihood ratio tests. The resulting ROC's are concave downward. The results from each simulation are above the a line of equality $\mathrm{P}_{\mathrm{D}}=\mathrm{P}_{\mathrm{FA}}$. The slope of the ROCs at a particular point is equal to the value of the threshold required to achieve the desired $P_{D}$ and $P_{F A}$ at that point. It is also observed that the area under the ROC increases as we increase the signal power. This indicates that the processor performance improves with increased signal power. The Matlab code which simulates these representative results is provided in Appendix $\mathrm{C}$. 


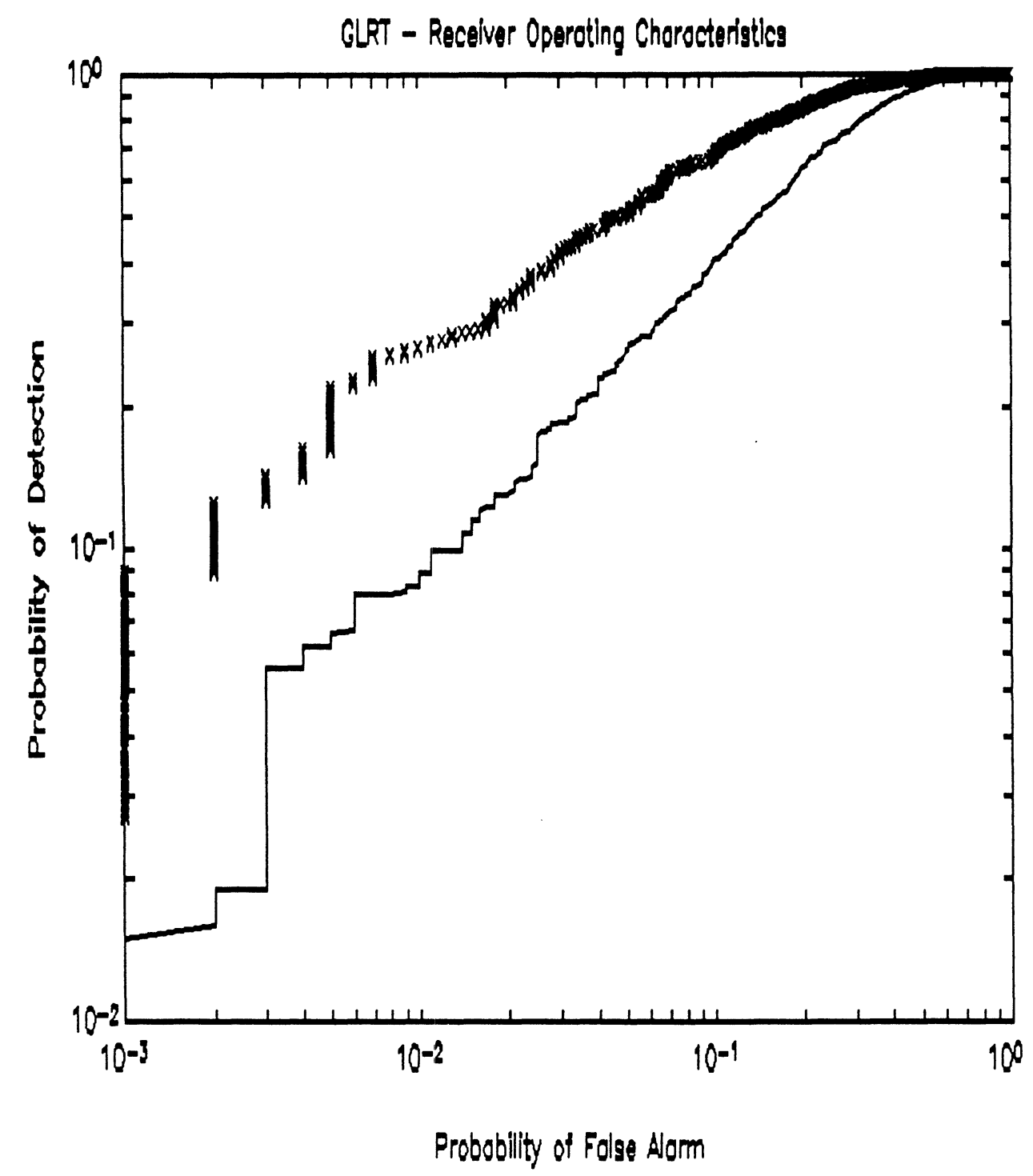

Figure 4.7 ROC Case 1: The jammer is located at 8 degrees.

$\begin{array}{lll}\text { The simulation parameters are: } & \text { Number of sensors: } & 8 \\ & \text { Null-to-null Beamwidth: } & 28.95 \text { degrees } \\ & \text { x denotes source power: } & -7.0 \mathrm{~dB} \\ & \text { - denotes source power: } & -8.0 \mathrm{~dB} \\ & \text { white noise power: } & -5.53 \mathrm{~dB}\end{array}$




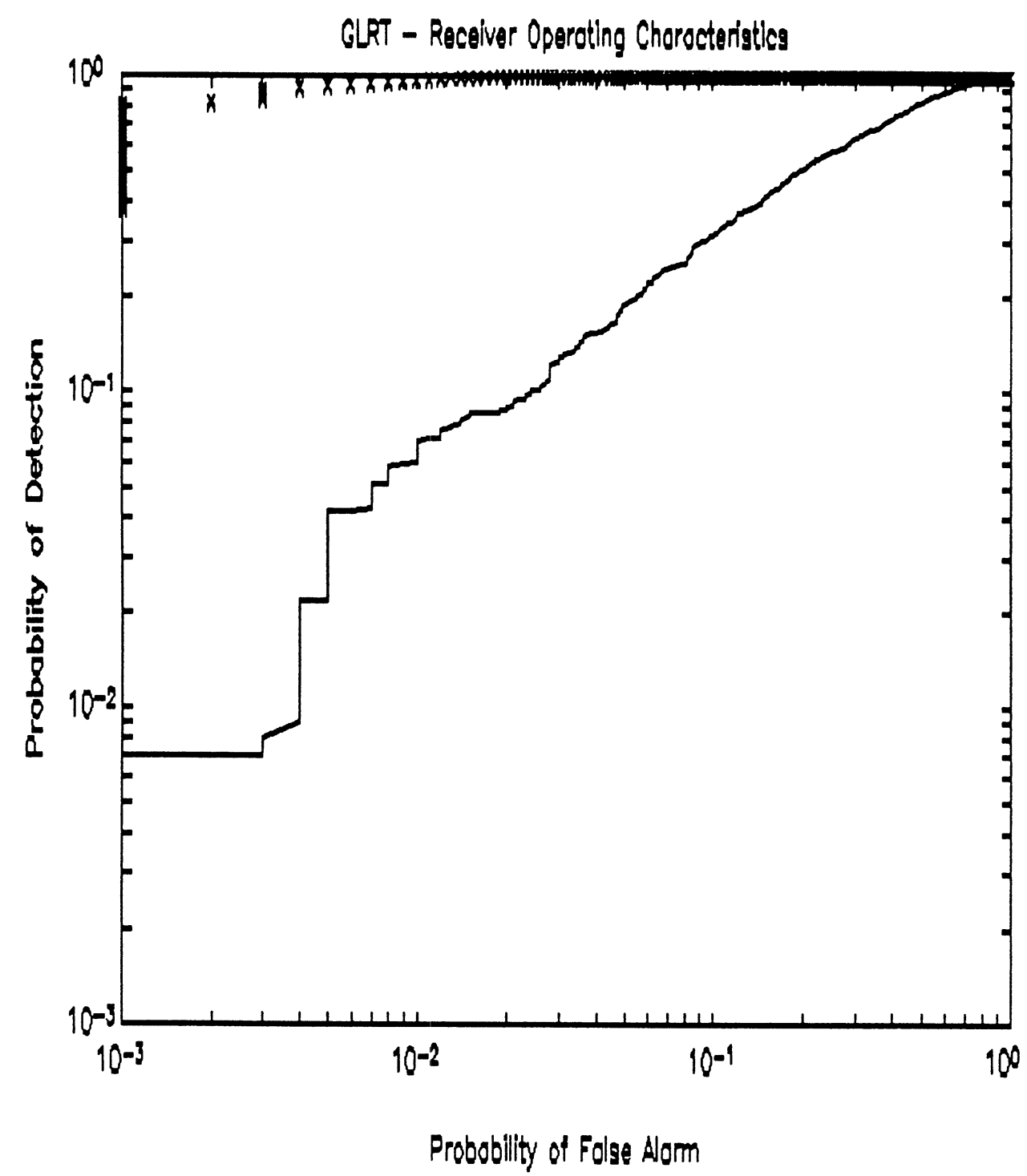

Figure 4.8 ROC Case 2: The jammer is located at 8 degrees.

The simulation parameters are: Number of sensors: 8 Null-to-null Beamwidth: 28.95 degrees $x$ denotes source power: - denotes source power: white noise power:

$-6.0 \mathrm{~dB}$

$-7.0 \mathrm{~dB}$

$-11.0 \mathrm{~dB}$ 


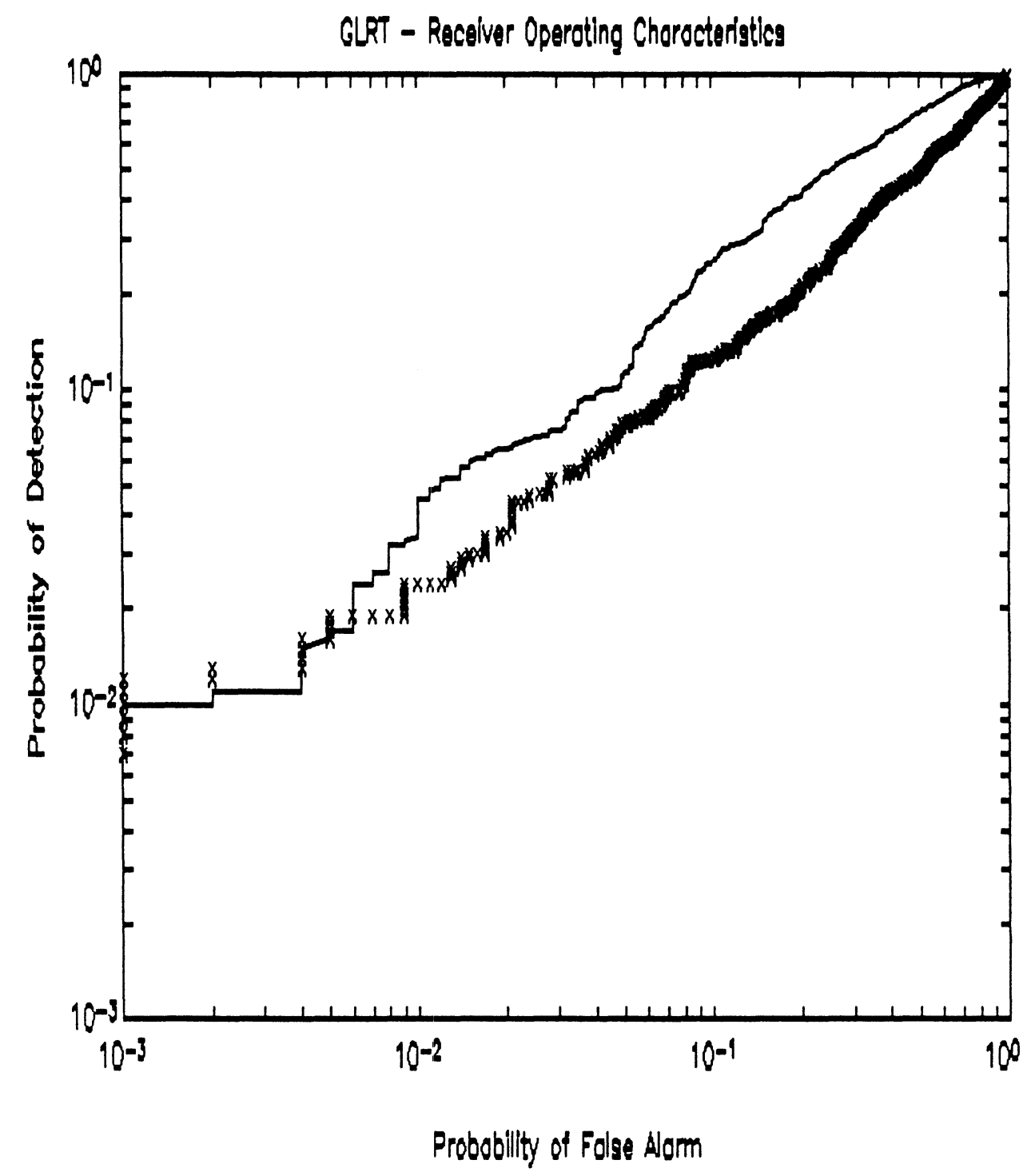

Figure 4.9 ROC Case 3: The jammer is located at 8 degrees.

The simulation parameters are: $\quad$ Number of sensors: 8

Null-to-null Beamwidth: $\quad 28.95$ degrees

$\mathrm{x}$ denotes source power:

$-1.0 \mathrm{~dB}$

- denotes source power:

$-2.25 \mathrm{~dB}$

white noise power:

$-5.53 \mathrm{~dB}$ 


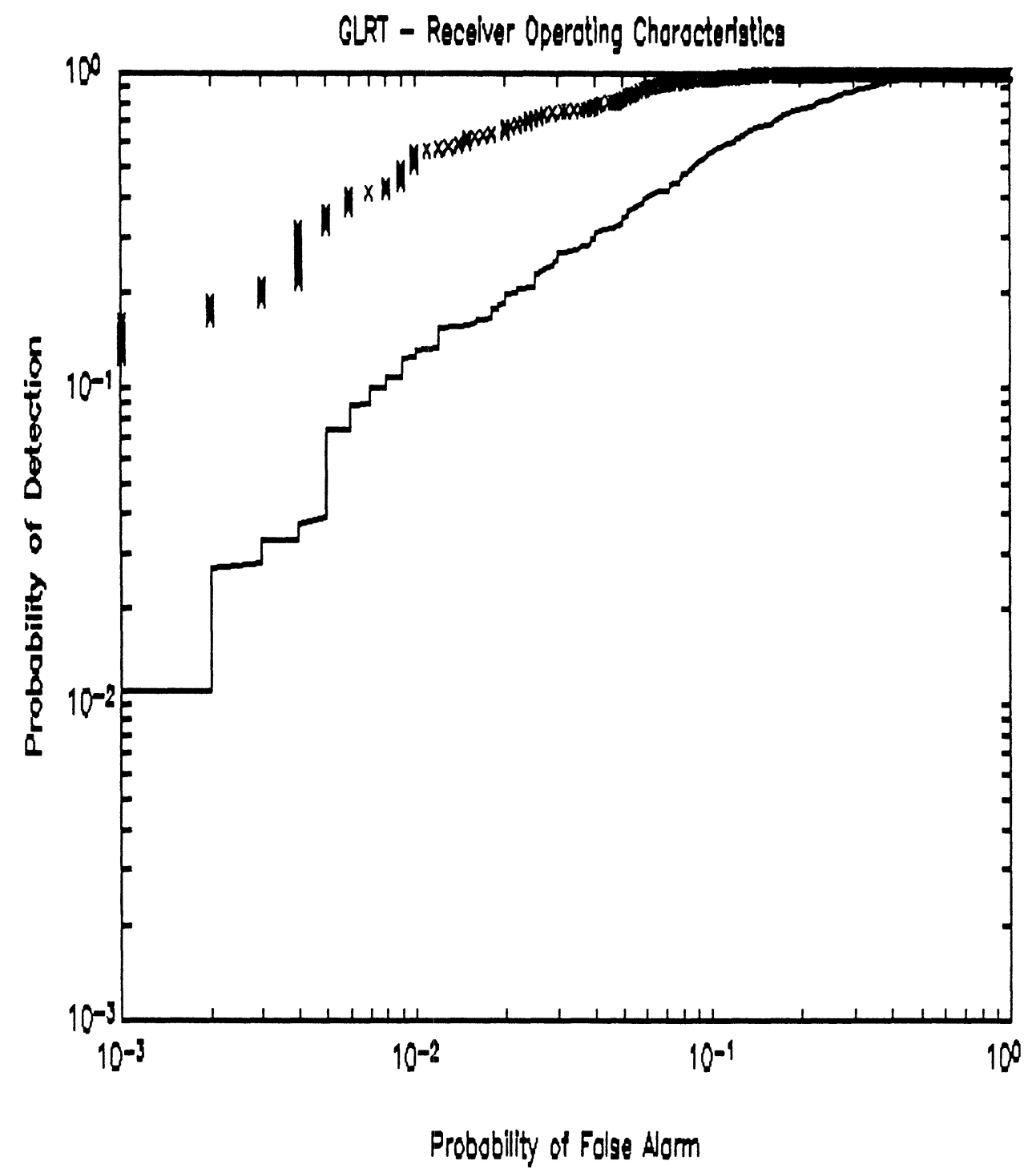

Figure 4.10 ROC Case 4: The jammer is located at 8 degrees.

The simulation parameters are: Number of sensors:

16

Null-to-null Beamwidth:

$\mathrm{x}$ denotes source power:

14.95 degrees

- denotes source power: white noise power:

$-5.0 \mathrm{~dB}$

$-7.25 \mathrm{~dB}$

$-11.0 \mathrm{~dB}$ 


\subsubsection{The GLRT As A Direction-of-Arrival Estimator}

The next part of this experiment is designed to estimate the unknown DOA. It requires that we specify a region of search to intelligently minimize the computational load. We choose the region of search to be \pm 5 degrees to minimize the computational load. This constraining dictates that we make an assumption about a priori source position in the ocean.

We model the array output and covariance matrix as described in equations (4.33) and (4.34). Table 4.1 summarizes the system parameters and the estimated DOA parameter. Each of the estimates were formed after conducting 200 Monte Carlo trials and the software code is provided in Appendix D. The estimated mean and variance for the DOA estimate $\hat{\theta}$ were computed using the following standard formulas:

$$
\hat{\theta}=\frac{1}{N} \sum_{i=1}^{N} \theta_{i}
$$

and

$$
\hat{\sigma}_{\theta}^{2}=\frac{1}{N} \sum_{i=1}^{N}\left(\theta_{i}-\hat{\theta}\right)^{2}
$$

where $\mathbf{N}=200$.

The results show that the GLRT yields more accurate estimates of the DOA as the signal power increases. In addition the respective variance decreases. For example, we examine the $-1 \mathrm{~dB}$ signal case and find a $35 \mathrm{x}$ increase in the estimated mean value between $\mathrm{N}=8$ and $\mathrm{N}=16$. 
Table 4.1 Direction of Arrival Parameter Estimation Summary

System Parameters

Estimated

DOA

\begin{tabular}{|c|c|c|c|c|c|c|c|c|}
\hline $\begin{array}{l}\text { Sig } \\
\mathrm{dB}\end{array}$ & $\begin{array}{l}\text { WGN } \\
\mathrm{dB}\end{array}$ & $\begin{array}{l}\text { Jam } \\
\mathrm{dB}\end{array}$ & $\begin{array}{l}\text { Sig } \\
(\theta)\end{array}$ & $\begin{array}{l}\text { Jam } \\
(\theta)\end{array}$ & $\begin{array}{c}\mathrm{N} \\
\text { Sensors }\end{array}$ & $\begin{array}{l}\text { Surveil } \\
\text { Region }\end{array}$ & $\begin{array}{l}\text { M } \\
\text { e } \\
\text { a } \\
\text { n } \\
\hat{\theta}\end{array}$ & $\begin{array}{l}\text { Var } \\
\text { of } \hat{\theta} \\
\hat{\sigma}_{\theta}^{2}\end{array}$ \\
\hline-12 & -11 & 0 & $0^{\circ}$ & $8^{\circ}$ & 16 & $-5^{\circ} \leq \theta \leq 5^{\circ}$ & 2.32 & 6.0177 \\
\hline-5 & -11 & 0 & $0^{\circ}$ & $8^{\circ}$ & 16 & $-5^{\circ} \leq \theta \leq 5^{\circ}$ & 0.36 & 1.92 \\
\hline-1 & -11 & 0 & $0^{\circ}$ & $8^{\circ}$ & 16 & $-5^{\circ} \leq \theta \leq 5^{\circ}$ & 0.07 & 0.6584 \\
\hline-12 & -11 & 0 & $0^{\circ}$ & $8^{\circ}$ & 8 & $-5^{\circ} \leq \theta \leq 5^{\circ}$ & 4.69 & 3.5978 \\
\hline-5 & -11 & 0 & $0^{\circ}$ & $8^{\circ}$ & 8 & $-5^{\circ} \leq \theta \leq 5^{\circ}$ & 3.89 & 2.3558 \\
\hline-1 & -11 & 0 & $0^{\circ}$ & $8^{\circ}$ & 8 & $-5^{\circ} \leq \theta \leq 5^{\circ}$ & 2.48 & 0.3356 \\
\hline & & & & & & & & \\
\hline
\end{tabular}




\subsection{GLRT Maxima Analysis}

The one-dimensional MFP plots provided in section 4.5 show that the output of the GLRT or statistic peaks when the hypothesized steering vector or replica field vector approximately matches the steering vector for the source of interest. In this section we conduct an analysis of the behavior of the eigenvalues which produced these results.

The one-dimensional GLRT indicated by equation (4.30) is directly dependent on the magnitude of the eigenvalues. These eigenvalues result from performing eigenvalue decomposition on the sample covariance matrices $\mathbf{S}_{0}$ and $\mathbf{S}_{1}$. By inspection of equation (4.30), the magnitude of the output statistic will be inversely proportional to the term raised to the $(N-r)$ power. Hence, when the denominator is small the resulting statistic will be large otherwise the statistic is large when the denominator is small.

We arbitrarily choose the system simulation parameters specified in ROC Case 2 (Figure 4.8) to simulate data to estimate $S_{o}$ and $S_{1}$. An eigendecomposition is computed on $S_{o}$ and $S_{1}$ to determine the signal and noise subspaces as:

$$
E\left\{S_{o}, S_{1}\right\}=U \Lambda U^{H}=\sum_{n=1}^{2} \lambda_{n} u_{n} u_{n}+\sigma^{2} \sum_{n=3}^{16} u_{n} u_{n}^{H}
$$

where the first term is the dominate subspace and the second term is the subdominant subspace. The GLRT is computed when the signal is modeled as present at array boresight and when it is absent from the array data. Figures (4.11) and (4.12) indicate these results. In both cases the jammer is accurately and clearly located at $8^{\circ}$ relative to a source present and not present at array boresight. Figure (4.11) contains two major 
peaks indicating detection of the source and jammer. We analyze the data of Figure (4.11) and determine that the weaker source location is estimated to be $-1^{\circ} \leq \theta \leq 0^{\circ}$.

Next we apply the a derivation of the law cosines to compute the projection of the replica field onto the signal subspace. We compute this projection using the formula:

$$
\begin{aligned}
\cos \theta & =\frac{\left|a^{H} b\right|}{\|a\|\|b\|} \quad \text { where, } \\
a & =s(\theta), \\
b & =P_{u 1, u 2} \cdot s(\theta) \quad \text { and, } \\
b & =P_{u 3 \ldots u 16} \cdot s(\theta) .
\end{aligned}
$$

The replica field vector is defined by $\mathbf{a}$, the signal plus jammer is defined by $\boldsymbol{P}_{u 1, u 2} \cdot s(\theta)$, and the interference only is defined by $\boldsymbol{P}_{u 3 \ldots u 16} \cdot s(\theta) . \boldsymbol{P}_{u 1, u 2}$ and $\boldsymbol{P}_{u 3 \ldots u 16}$ are projection matrices. Equation (4.40) projects the vector $\mathbf{s}(\theta)$ onto the subspace spanned by the replica vector [53]. Figure (4.13) depicts the results when we consider the signal subspace. We note that the replica field vector and signal vector are close to being orthonormal when the replica field vector "matches" the signal vector. This indicates the best low rank 2 approximation to the data when the signal is present. This procedure is repeated to compute projection of the replica field vector onto the noise subspace. Figure (4.14) depicts these results. In this case we are essentially searching for an orthonormal projection in the noise subspace using the replica field vectors. Here when the replica field vector "matches" that of the jammer component in the noise subspace we observe nulls in the processed data at approximately $\theta=0^{\circ}$ and $\theta=8^{\circ}$. 
We continue this analysis by computing the eigendecomposition of $\boldsymbol{P}^{\perp} \boldsymbol{S}_{1} \boldsymbol{P}^{\perp}$ retaining the largest eigenvalue for each projection matrix $\boldsymbol{P}^{\perp}$. The projections have a one-to-one correspondence to the replica field in the $-30^{\circ} \leq \theta \leq 30^{\circ}$ degree field of view. Thus we compute 61 eigendecompositions. The algorithm requires pre multiplying and post multiplying $S_{1}$ by $\boldsymbol{P}^{\perp}$ to remove the estimated low rank interference. Since we assumed a rank 1 complex signal, we can now construct the best low rank approximation of the data absence of the jammer and background noise. Figure 4.15 indicates the changes in the largest eigenvalue of $P^{\perp} S_{1} P^{\perp}$ verses the assumed DOA. This approach also allows the white noise levels to be estimated. Figure 4.16 displays the white noise estimate under $\mathrm{H}_{1}$ verses the assumed DOA. The white noise estimate is $\hat{\sigma}_{w}^{2}=0.0803865$ under $\mathrm{H}_{\mathrm{o}}$. At the start of this simulation the white noise level was specified to be $\sigma_{w}^{2}=0.07943282$. We conclude the white noise estimate contains a portion of source energy and is too large. Therefore, we should accept the $\mathrm{H}_{1}$ hypothesis and reject the $\mathrm{H}_{\mathrm{o}}$ hypothesis. This simulation results indicated in Table 4.2 agree with the prescribed scenario of ROC Case 2. The software code used to compute these results is provided in Appendix E.

Table 4.2 White Noise Estimates

\begin{tabular}{||l|l|l|}
\hline DOA $\left(\theta^{\circ}\right)$ & $\mathrm{H}_{\mathrm{o}}: \max \sigma_{\mathrm{w}}{ }^{2}$ & $\mathrm{H}_{1}: \max \sigma_{\mathrm{w}}{ }^{2}$ \\
\hline $0^{\circ}, 8^{\circ}$ & - & $0.076286,0.074512$ \\
\hline$-30^{\circ} \leq \theta \leq 30^{\circ}$ & $\begin{array}{l}0.08038685 \\
\text { (constant) }\end{array}$ & - \\
\hline
\end{tabular}




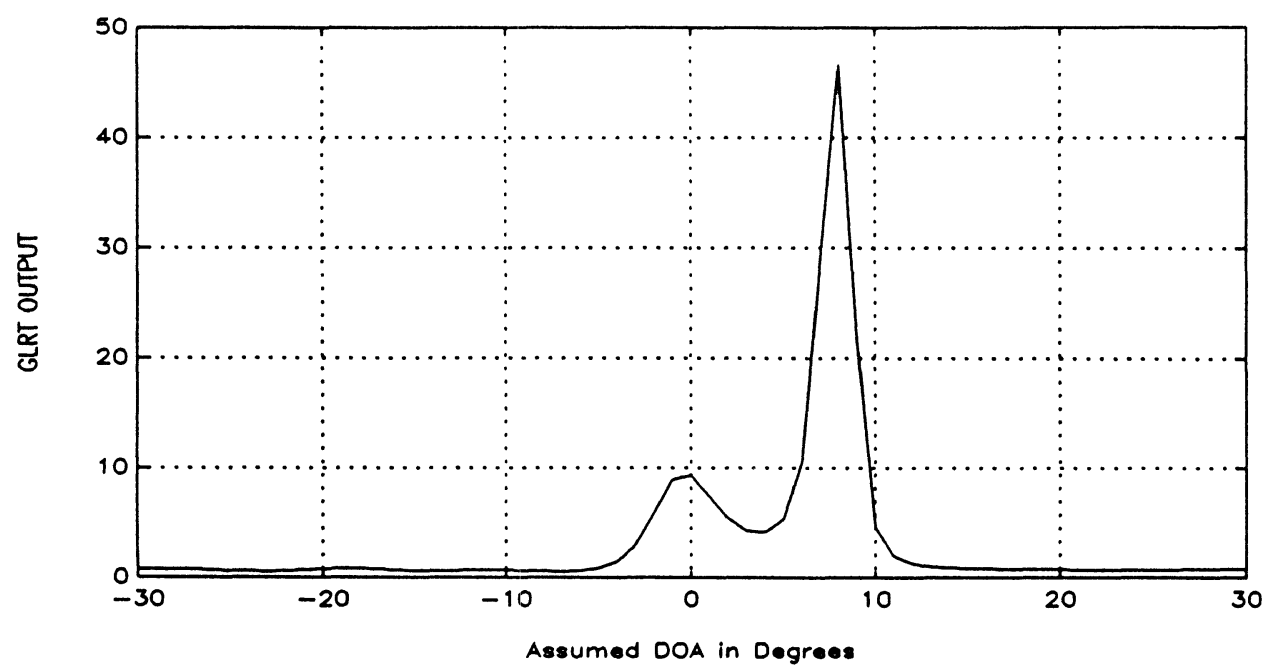

Figure 4.11 GLRT output as replica spans field of view $\left(\mathrm{H}_{1}\right.$ assumption)

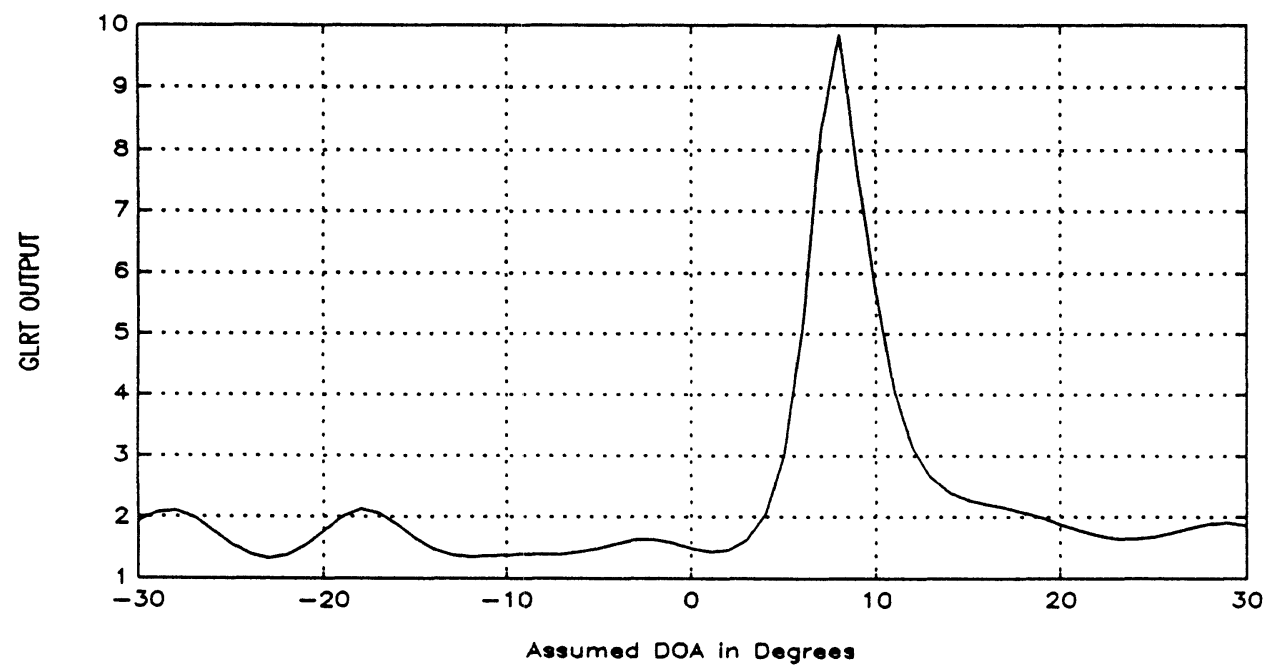

Figure 4.12 GLRT output as replica spans field of view ( $\mathrm{H}_{0}$ assumption) 


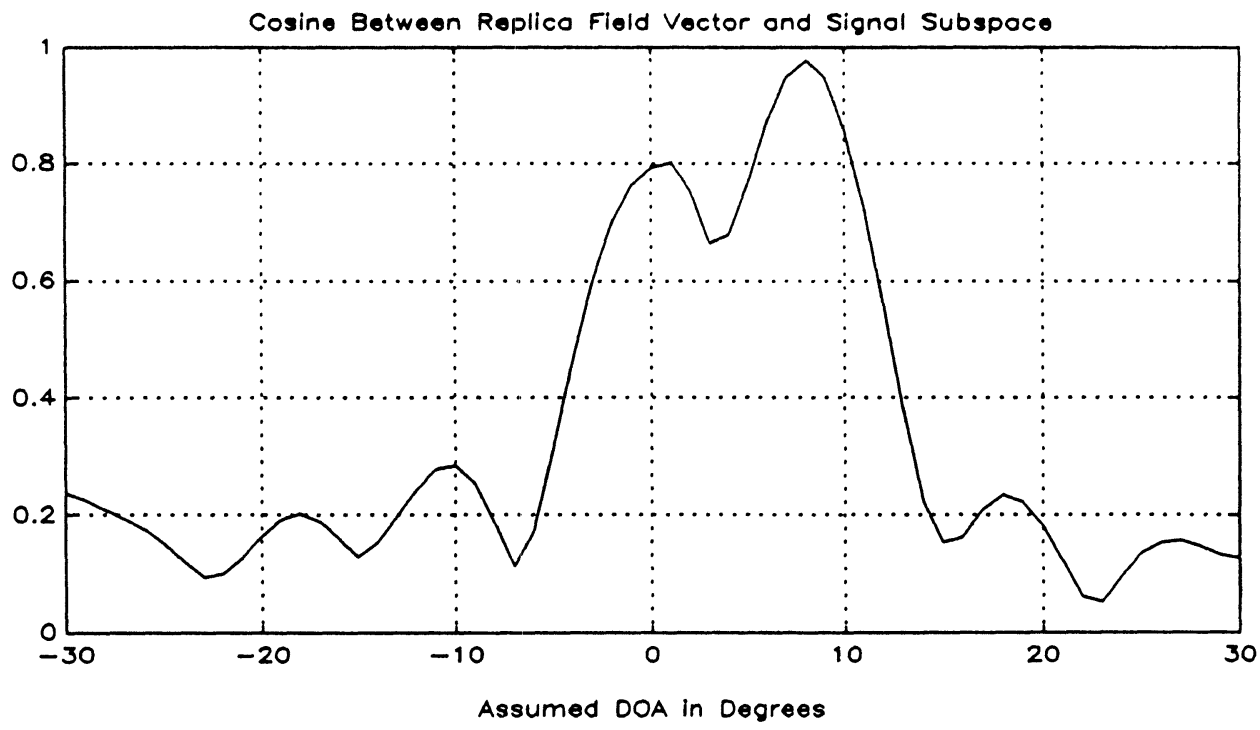

Figure 4.13 Replica vector projected on to signal subspace

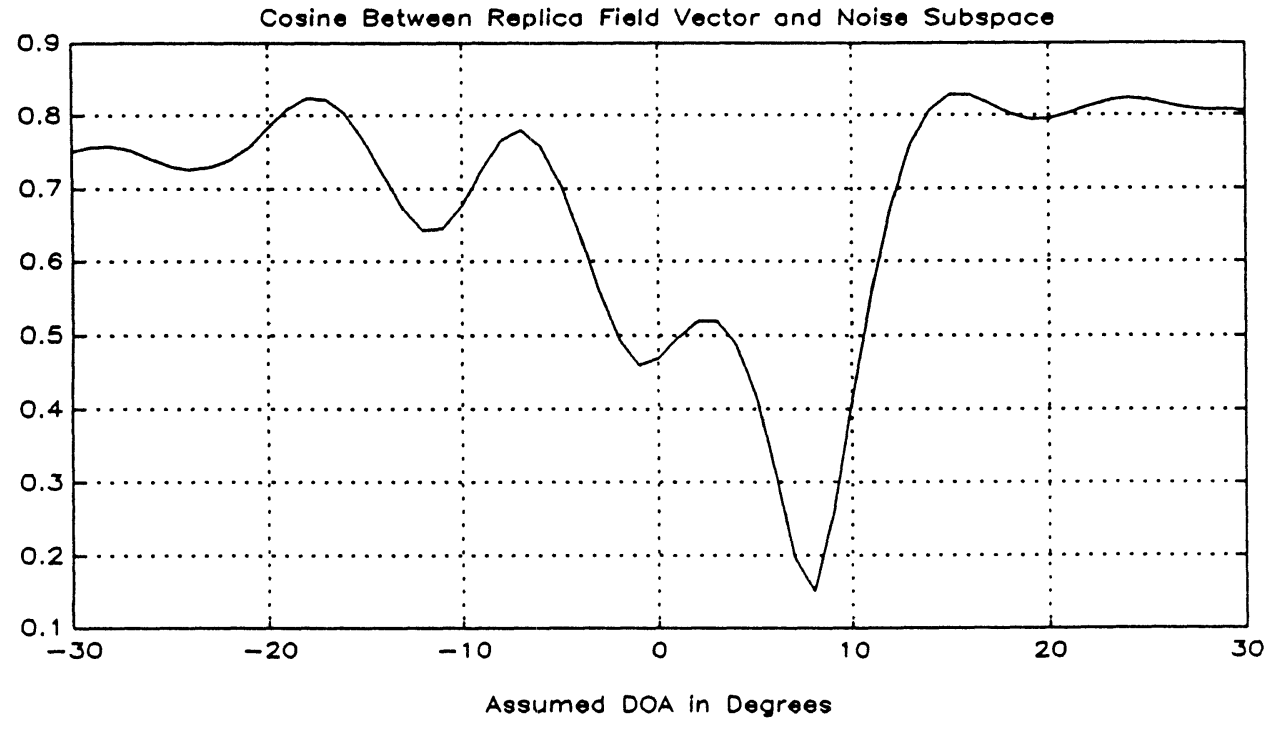

Figure 4.14 Replica vector projected on noise subspace 


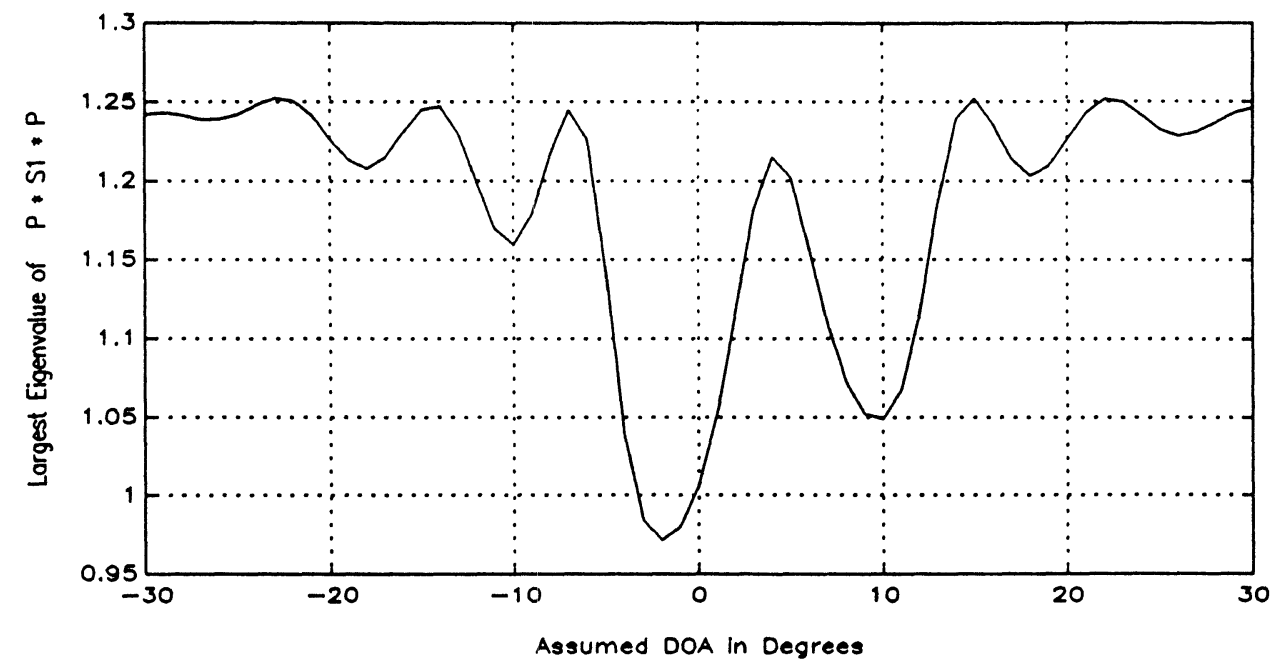

Figure 4.15 Changes in largest eigenvalue under $H_{1}$ assumption

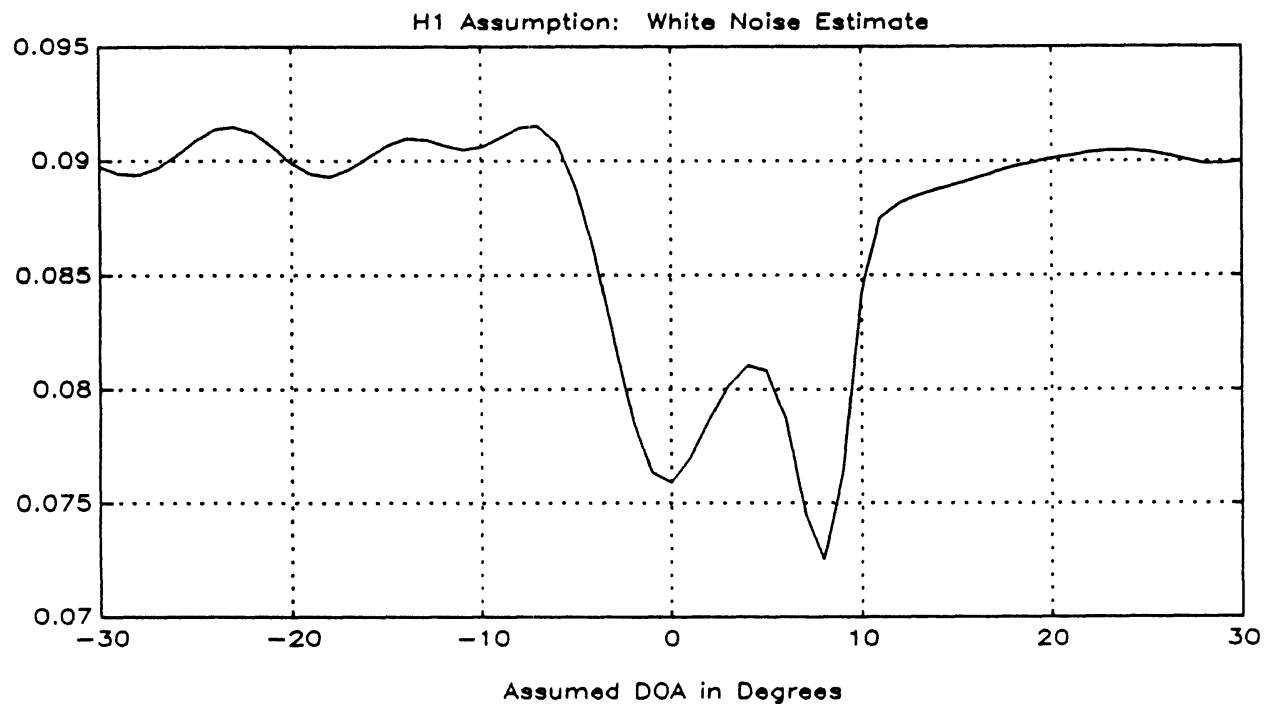

Figure 4.16 White noise estimate under $\mathrm{H}_{1}$ assumption 


\section{CHAPTER 5 \\ SIMULATIONS FOR MATCHED-FIELD SOURCE LOCALIZATION}

\subsection{Introduction}

This chapter presents representative results of a matched field source localization experiment. A description of the problem parameters and the simulation procedures are included. This chapter commences with the Bartlett estimator results, the MLM estimator results, and the GLRT algorithms to detect and localize the source in the presence of a strong low rank jammer. An evaluation of the three approaches is also provided.

\subsection{Problem Formulation}

We present the scenario and simulation of the data based on the parabolic equation acoustic model described in Section 3.3.2 and Section 3.4. This model is employed to rapidly compute three-dimensional acoustic field points in a complex ocean environment. All experiments are conducted assuming shallow water environments. Several software models in Appendices $\mathrm{F}, \mathrm{G}$, and $\mathrm{H}$ compute the $N \times 2 D$ representation for the three dimensionally varying environment as if each slice of the sound speed profile were derived from a cylindrically symmetric problem. The selected geoacoustical parameters for the environment are displayed in Figure 5.1 and described in the following paragraph.

We model an array comprised of 32 equally spaced sensors in a vertical configuration. The spacing between sensors is 5 meters and the first sensor is placed at zero meters relative to the search domain. The search domain is indicated by the dot- 
filled box, and the vertical array position to the left as shown in Figure (5.1). The water is 500 meters deep. This array provides a depth coverage of 160 meters. The source and jammer are presumed to be in the near-field and choose a 200 meter range by 300 meter depth search domain. The replica field vectors are computed in 5 meter range increments and 5 meter depth increments. The sound velocity profile is shown on the left side of the diagram. It is reasonable to linearize the sound speed profile for shallow water environments since the deep sound channel does not usually exist at these depths. We have modeled a non-sloping bottom with attenuation set to $0.4 \mathrm{~dB} / \lambda$. Initially, the source and jammer are modeled as being located at $(r=100 \mathrm{~m}, z=70 \mathrm{~m})$ and $(r=190 \mathrm{~m}$, $z=100 \mathrm{~m})$. respectively. These parameters are specified through an input data file which is read by the P/E solver to create the scenario of Figure 5.1. 


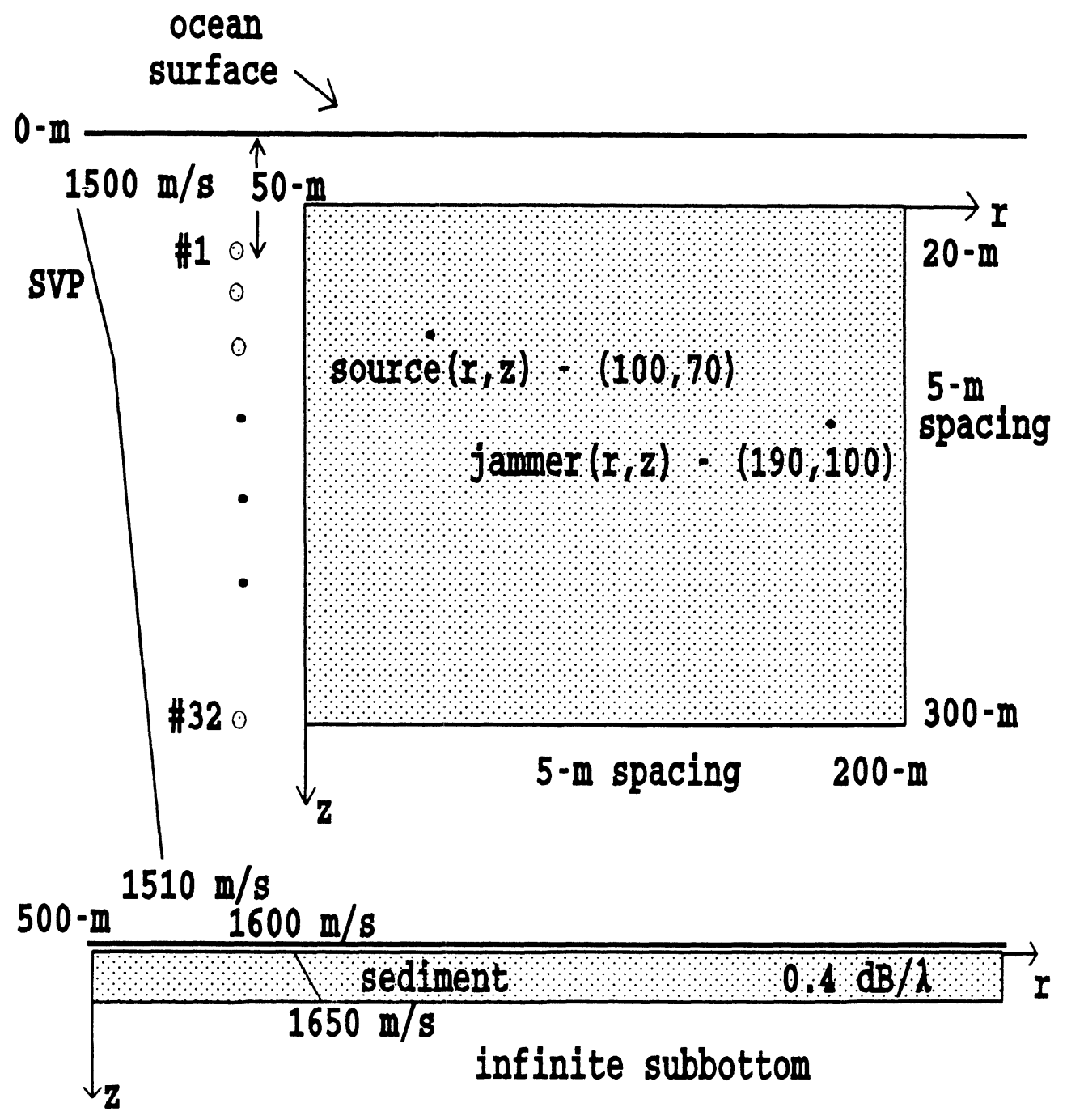

Figure 5.1 Geoacoustics of the environment used for simulations 


\subsection{The Bartlett Estimator}

This section describes the results of the Bartlett estimator which has been embedded directly into the P/E solver code. These fields are computed concurrently in the P/E solver code, the Bartlett estimator calculations are performed, and the resulting ambiguity surface data are stored for subsequent display.

The Bartlett estimator is evaluated as:

$$
P_{B a r t}(e, r)=e(r, z) R e(r, z),
$$

where $\mathbf{e}$ is the replica field. We use the actual correlation matrix $\mathbf{R}$ which is defined as:

$$
\hat{R}=\sigma_{s}^{2} e_{s} e_{s}^{H}+\sigma_{j}^{2} e_{j} e_{j}^{H}+\sigma_{n}^{2} I
$$

The vector array measurements for source and jammer are denoted by $\mathbf{e}_{\mathbf{s}}$ and $\mathbf{e}_{\mathbf{j}}$ respectively and computed using the $\mathrm{P} / \mathrm{E}$ solver in Appendix $\mathrm{F}$. We assume two incoherent targets (source plus jammer), to alleviate the cross terms when expanding equation (5.1) with equation (5.2) substituted. It is easy to show that Equation (5.3) is a convenient implementation of the Bartlett estimator which leads to efficient software coding.

$$
P_{B a r t}(r, z)=\sigma_{s}^{2}\left|e^{H}(r, z) e_{s}\right|^{2}+\sigma_{j}^{2}\left|e^{H}(r, z) e_{j}\right|^{2}+\sigma_{n}^{2} I
$$


The replica vectors, source, and jammer vectors are normalized by their respective unit length, i.e., $e_{n}=\frac{e}{\|e\|}$ as a standard practice and to better enable comparison of the data and replica fields. We include in equation (5.4) ambient background noise, a zero-mean, Gaussian process with covariance matrix $\sigma_{n}^{2} I$. Next we compute a desired $\sigma_{\mathrm{n}}^{2}$.

The signal-to-white noise ratio (SNR) is defined as:

$$
\begin{aligned}
S N R & \triangleq \frac{\|s(t)\|^{2}}{\|n(t)\|^{2}} \\
& =10 \cdot \log _{10}\left[\frac{|\boldsymbol{P}|^{2}}{N \cdot \sigma_{n}^{2}}\right] \\
\Rightarrow \sigma_{n} & =\frac{|\boldsymbol{P}|^{2}}{\sqrt{N}} \cdot 10^{-(S N R / 20)},
\end{aligned}
$$

where $\mathbf{P}$ is the complex pressure vector computed by the $\mathrm{P} / \mathrm{E}$ Solver and $\mathrm{N}$ is the number of hydrophones. The jammer-to-noise ratio (JNR), $\sigma_{j}^{2}$, is defined in a similar manner to equation (5.5).

We specify a signal power of $-1.01 \mathrm{~dB}$, a jammer power of $1.0 \mathrm{~dB}$, and a background noise level of $-11.0 \mathrm{~dB}$ together with the parameters specified in Figure 5.1 and run the P/E solver plus Bartlett estimator. The P/E Solver plus Bartlett estimator code is provided in Appendix F. The ML estimator requires that the covariance matrix $\mathbf{R}$ be inverted and we apply the standard algorithms as prescribed by Golub and Van Loan [54]. The search domain is subdivided into 1160 hypothesized source locations and 
the resulting ambiguity surface is stored in a data file named bartlett.dat. An analysis/display program was written to read the data file and the results are provided in Figure 5.2. This display program (surf.pro) is provided in Appendix $\mathbf{G}$ and provides good analysis display capability when the data is output to a color monitor. This display requires thresholding; and we discard data below the magnitude of 1.1. Figure 5.3 shows the data that is above the threshold and we conclude that detecting and localizing a source under the previously specified conditions would be nearly futile when using mesh. We continue the analysis of the Bartlett ambiguity surface by using the intensity color shading capabilities of display program surf.pro in conjunction with the color monitor, and conclude that peaks occur in the proper locations. The numerical results for range and depth estimates are summarized as part of Section 5.6. 


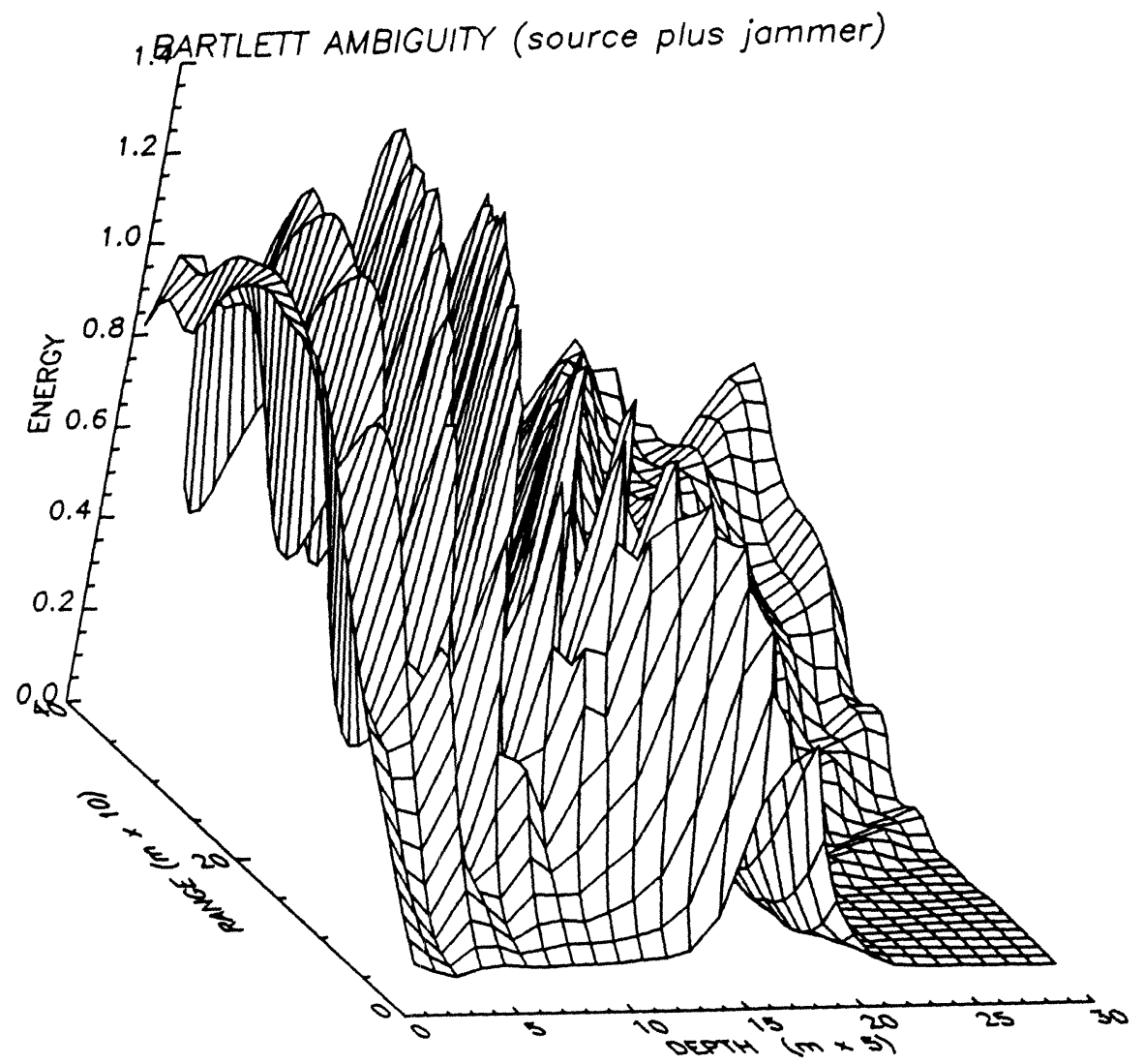

Figure 5.2 MFP Case 1: Bartlett Ambiguity Surface, No Thresholding 


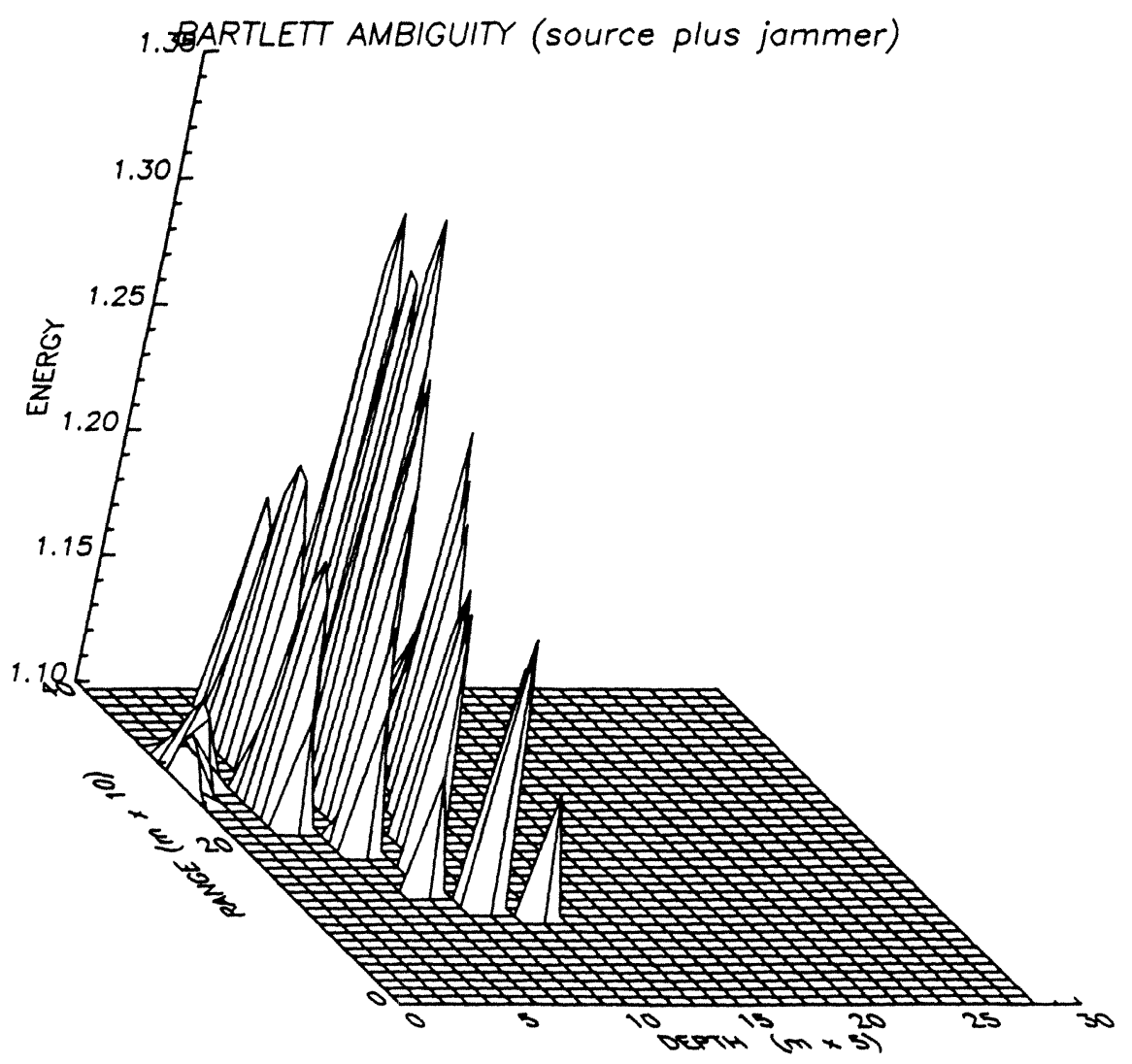

Figure 5.3 MFP

$$
\text { MFP Case 2: Bartlett Ambiguity Surface, Threshold }=1.1
$$




\subsection{The Maximum Likelihood Estimator}

This section describes the ML estimator which has been embedded directly into the $\mathrm{P} / \mathrm{E}$ solver code. The mathematical definition follows that of the Bartlett estimator described in section 5.3 and similarly handles the source replica field and jammer replica field. These fields are computed concurrently with the P/E solver, the ML estimator computations are performed, and the ambiguity surface data are stored for subsequent display.

The maximum likelihood methods computes

$$
P_{M L}(r, z)=\left[e^{H}(r, z) R^{-1} e(r, z)\right]^{-1}
$$

where $\mathbf{e}(r, z)$ is the replica field. We use the actual correlation matrix $R$ which is defined as:

$$
\hat{R}=\sigma_{s}^{2} e_{s} e_{s}^{H}+\sigma_{j}^{2} e_{j} e_{j}^{H}+\sigma_{n}^{2} I
$$

Software which implements this $\mathrm{ML}$ estimator is provided in Appendix $\mathrm{F}$. The simulation parameters specified in section 5.2 and Figure 5.1 are unchanged. The search domain remains fixed and the P/E Solver-MLM output is stored in mlm.dat We repeat the procedure of reading the ambiguity surface data file (mlm.dat) and displaying it with the surf.pro software routine provided in Appendix G. Figures 5.4 and 5.5 show the ML ambiguity surfaces without thresholding and with a threshold value $=0.4$ respectively. These figures demonstrate that the ML estimator provides greater resolution than the Bartlett. We are able to use the identical 'mesh' feature and inspect the plots to 
determine the location of the source and jammer with increase accuracy. The peaks appear more pronounced and the sidelobe levels have been decreased.

We repeat the data analysis using the color intensity features of surf.pro and the color monitor to determine the location of the source and jammer. The numerical localization results for range and depth are summarized as part of Section 5.6. 


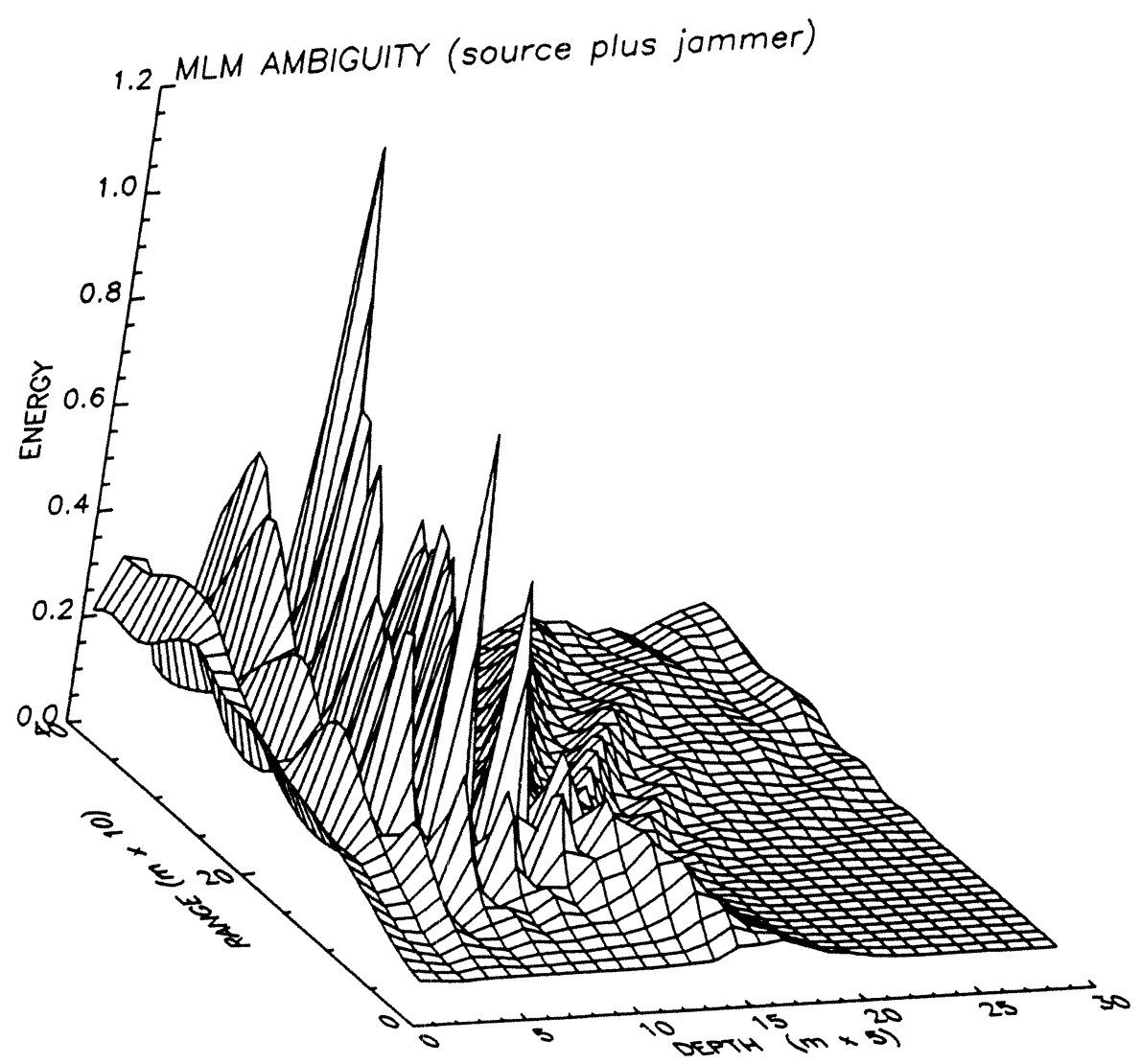

Figure 5.4

$$
\text { Case 3: MLM Ambiguity Surface, No Thresholding }
$$




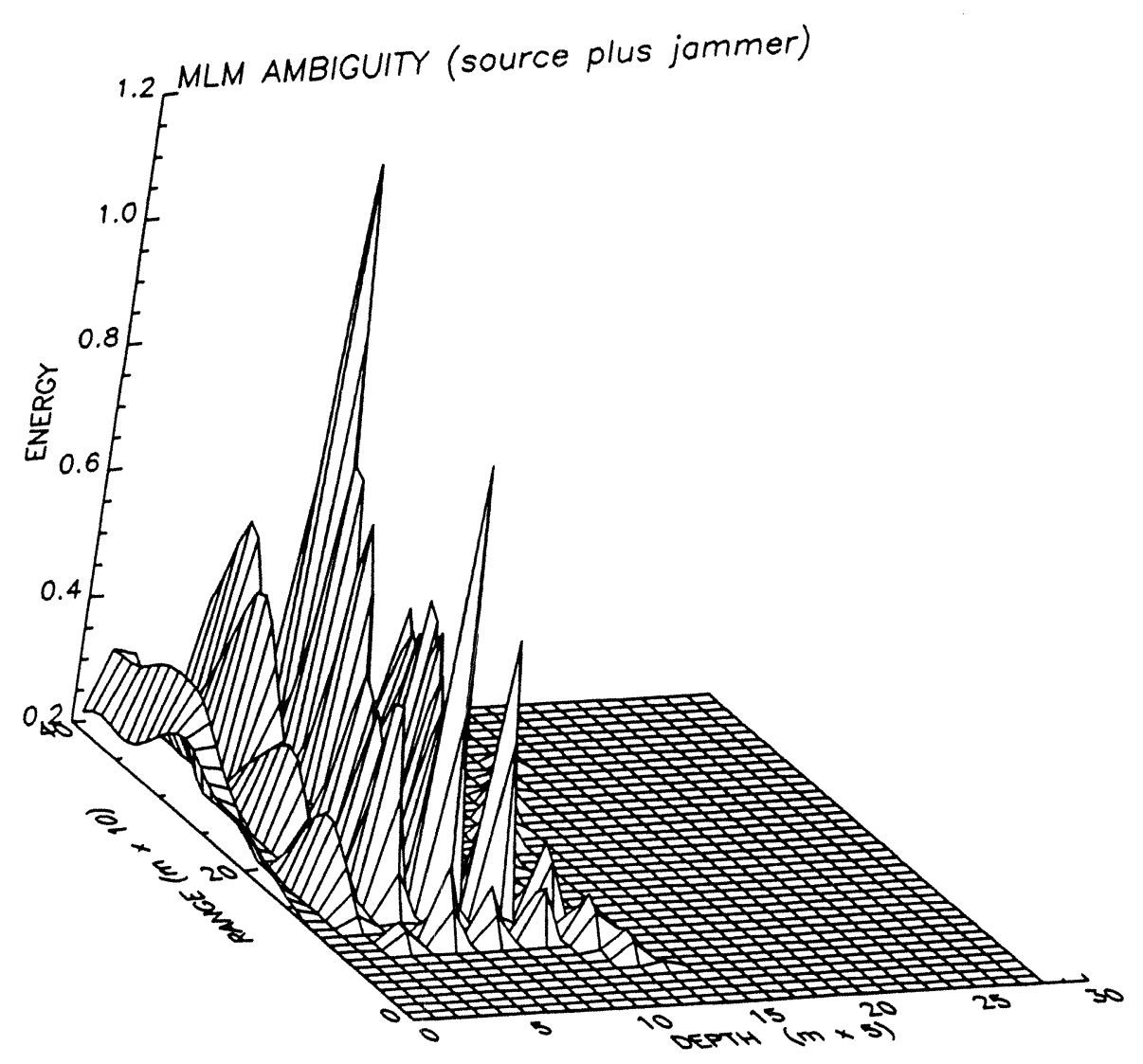

Figure 5.5 MFP Case 4: MLM

$$
\text { iguity Surface, Threshold }=0.2
$$




\subsection{The Generalized Likelihood Ratio Test}

This section describes the results of the GLRT algorithm. We apply the mathematical formulation of the GLRT as presented in Section 4.5. We describe the simulation procedures which differ from the Bartlett and MLM estimator. Examples of ambiguity surfaces produced from the GLRT are described.

We execute the P/E Solver on a stand-alone basis using the geoacoustical parameters in Figure 5.1 to simulate the vertical array data and the replica fields which model the source and jammer. The SNR and JNR remain fixed at $-10 \mathrm{~dB}$ and $-12 \mathrm{~dB}$ respectively. The P/E Solver is executed once with the source present in the ocean environment to simulate data under the $\mathrm{H}_{1}$ hypothesis and once with the signal absent to simulate data under the $\mathrm{H}_{\mathrm{o}}$ hypothesis. Minor modifications were made to the $\mathrm{P} / \mathrm{E}$ solver code in Appendix B to store the replica and array data. In this approach we average over simulated snapshots to estimate the correlation matrix. This infuses the signal information into the GLRT which is necessary information to localize the source.

Next, we read the data simulated under $\mathrm{H}_{1}$ for processing with the GLRT algorithm. The GLRT algorithm computes the z-statistic verses range verses depth and displays it as a three-dimensional surface using the 'mesh' feature. The code used to compute the GLRT output and display the data is provided in Appendix H. Figure 5.5 is the output of the GLRT when the source plus jammer are modeled. Although the jammer power is greater than the source power, we determine from inspection that GLRT is able to detect and localize the source. This fact is indicated by the two major peaks in Figure 5.5 which is an analysis of the simulated data under the $\mathrm{H}_{1}$ hypothesis. 
We threshold the data below a magnitude of 500 and display this ambiguity surface in Figure 5.6. Figure 5.6 provides an even better detection and localization result then the ML ambiguity surface in Figure 5.4. We conclude the analysis of the scenario described in Section 5.1 with the GLRT processed data which was simulated under the $\mathrm{H}_{\mathrm{o}}$ hypothesis. The results in Figure 5.7 clearly show that the strong low rank jammer has been detected an localized when the signal is not present. 


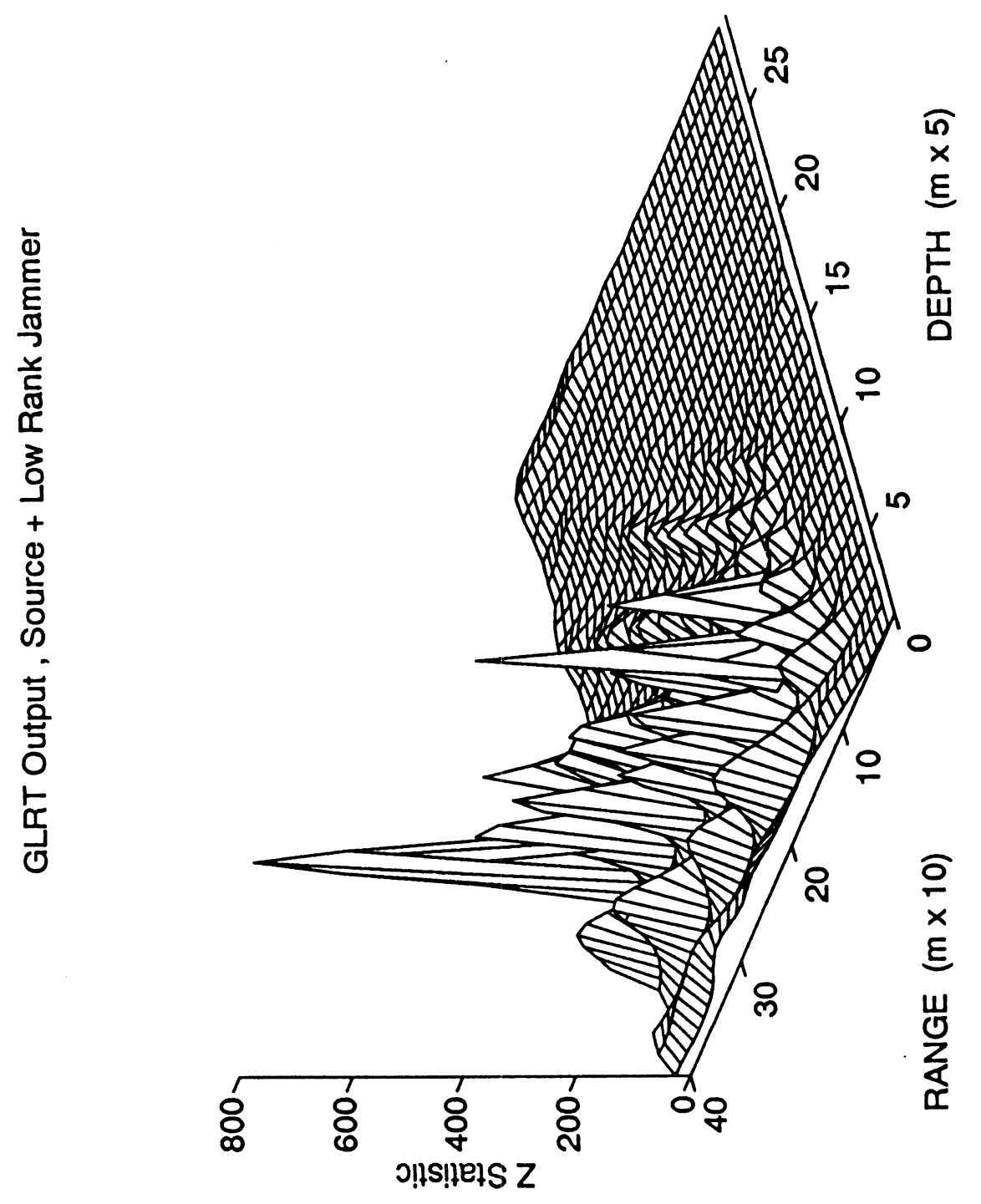

Figure 5.6 MFP Case 5: GLRT Ambiguity Surface, Source Plus Jammer, Threshold $=0.0$ 


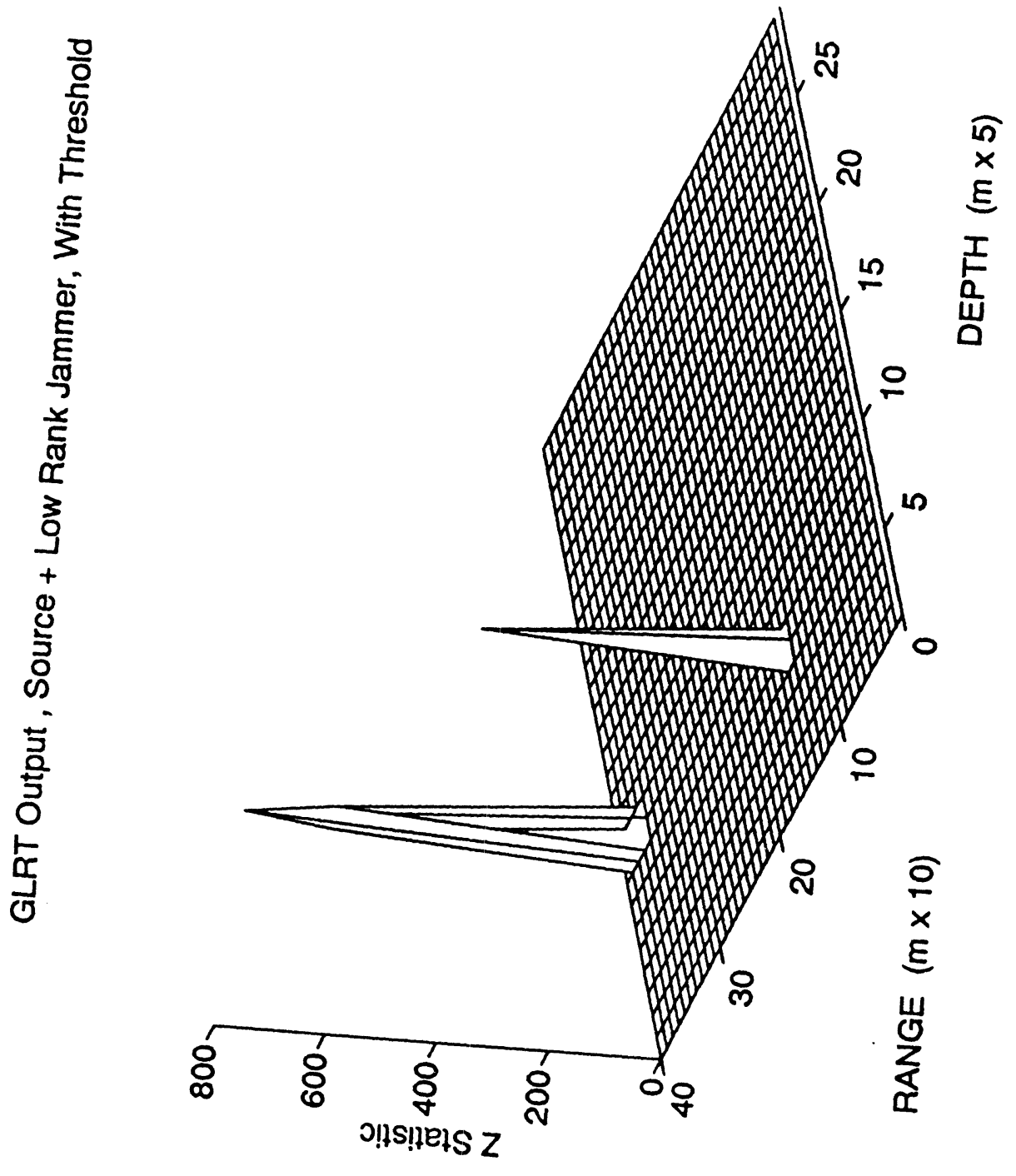

$\begin{array}{ll}\text { Figure 5.7 } & \text { MFP Case 6: GLRT Ambiguity Surface, Source Plus Jammer, } \\ & \text { Threshold }=500\end{array}$ 


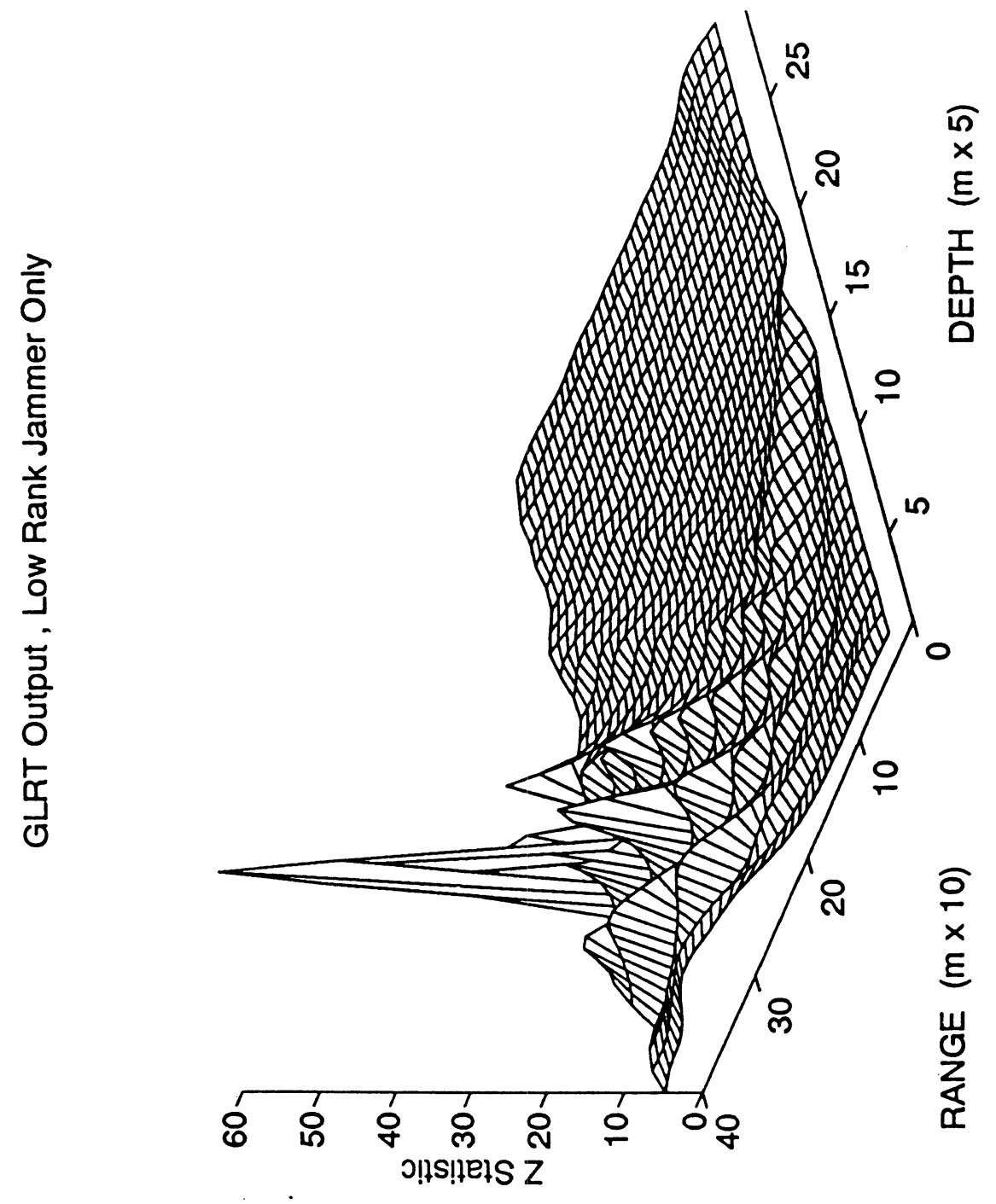

Figure 5.8 MFP Case 6: GLRT Ambiguity Surface, Low Rank Jammer, Threshold $=0.0$ 


\subsection{Summary of Simulations}

This section presents estimated range and depth parameters which were obtained by implementing the Bartlett estimator, the ML estimator, and the GLRT algorithm. The results are tabulated for comparison to the initial modeling parameters.

The data sets from each of the above localization schemes are searched for the largest amplitudes and the corresponding range depth coordinates. The results are tabulated in Table 5.1. We determine that the ML estimates offer improvement over the Bartlett estimates and this agrees with theory presented in Chapter 2. We conclude from this set of experiments as well as others that the most accurate results are produced by the GLRT algorithm.

Table 5.1 Summary of Estimated $(r, z)$ Parameters

\begin{tabular}{||l|l|l|l||}
\hline \hline Source Model (r,z) & Bartlett & MLM & GLRT \\
$r=75 \mathrm{~m}$ & $\hat{r}=84 \mathrm{~m}$ & $\hat{r}=74 \mathrm{~m}$ & $\hat{r}=75 \mathrm{~m}$ \\
$d=70 \mathrm{~m}$ & $\hat{d}=100 \mathrm{~m}$ & $\hat{d}=76 \mathrm{~m}$ & $\hat{d}=70 \mathrm{~m}$ \\
& & & \\
\hline Jammer Model (r,z) & Bartlett & MLM & GLRT \\
$r=190 \mathrm{~m}$ & $\hat{r}=180 \mathrm{~m}$ \\
$d=100 \mathrm{~m}$ & $\hat{d}=100 \mathrm{~m}$ & $\hat{r}=184 \mathrm{~m}$ & $\hat{r}=190 \mathrm{~m}$ \\
& & $\hat{d}=100 \mathrm{~m}$
\end{tabular}




\subsection{Critical Evaluation Of The GLRT}

This section describes the strengths and limitations of the GLRT. We discuss

these items from an implementation as well as a practical point of view.

The technological benefits this approach to MFP problems are:

(1) The GLRT is a detector. It can be used to detect the presence and estimate the parameters of target signals embedded in background ocean noise. It inherently detects all desired and undesired targets and is CFAR.

(2) This approach exploits a priori information. Benefits are offered since the is we do take advantage of the partial information available specifying covariance structure.

(3) This approach offers superior localization capability as opposed to the conventional Bartlett and maximum likelihood methods.

(4) This approach is not limited to specific signal models. We conducted the experiments using the Gaussian signal model but deterministic or random signal models can be used.

The technological limitations this approach to MFP problems are:

(1) The GLRT is computational intensive. It requires, for each output statistic, the eigendecomposition of two matrices. The dimension of the matrices are dependent upon the number of sensors in the array and computations are expensive for large arrays.

(2) There must be a priori information regarding the source location. In order to specify a "reasonable" search domain, information is required to intelligently specify the size and increments required for replica field simulations

(3) This methodology requires a priori information regarding the rank of interference. This approach allows for modifications of the assumed rank through repeating the entire detection, parameter estimation, and location process. This methodology can produce good results when a priori information regarding the rank is unavailable but the computational costs may be expensive. 


\section{CHAPTER 6 CONCLUDING REMARKS}

\subsection{Conclusions}

This dissertation established an investigation of matched field processing which started with conventional methods such as the Bartlett and ML estimators. The central issues were to include strong low rank jammer into the matched field scenario and develop a signal processing methodology to detect and localize the source in the presence of the directional interference. The latest acoustical modeling technology such as the Parabolic Equation method for approximating solutions to the wave equation were applied. This approach allowed for the prediction of sound in a complex ocean environment when range dependent conditions are assumed. We were able to successfully demonstrate the conjectured proof of concept that the GLRT can be a viable approach to detecting and localizing targets in high noise, range dependent environments. This was successfully accomplished by applying concepts from linear algebra, multivariate statistics, and reduced rank signal processing. The following paragraphs summarize the conclusions drawn from major components of these experiments.

As a basis for problem formulation, we selected and demonstrated two sophisticated methodologies for ocean environmental modeling. The normal mode method was demonstrated as an effective approach to modeling acoustic pressure in complex range dependent ocean environments and the parabolic equation was demonstrated as a viable approach to modeling acoustic pressure in complex range independent environments. It was determined that normal mode model provides 
sufficiently accurate results in range dependent environments when the area of interest is properly segmented or the geoacoustical parameters are weakly range dependent.

The ML estimator was shown to exhibit superior source localization capabilities verses the Bartlett estimator primarily because of lower sidelobes around the main peaks. The ML estimator produces a richer ambiguity surface. It was feasible to visually inspect MLM and GLRT outputs to determine the range and depth values. Often the Bartlett output provided little range and depth information from a visual perspective. It should be noted that a computational trade of is required in the form of inverting the covariance matrix $\mathbf{R}$. We conclude that the ML estimator is the more favorable approach given the benchmarks of current central processing units (cpu's) and the efficient matrix inversion algorithms. The ML estimator is suggested as a first order matched field processor which would be useful in selecting a first order surveillance domain. Upon selection of the surveillance domain, more sophisticated and expensive signal processing method can be applied such as the GLRT.

The GLRT was derived and shown to be a useful approach for detecting signals that include unknown parameters. This derivation followed ML estimation constraints which are not thoroughly addressed in the statistical literature. Specifically our problem required, the joint estimation of an unknown mean vector and covariance matrix with rank $r$ when there is not enough information to derive the ML estimators. However through several tedious and rigorous mathematical approaches the final result given by the test statistic in equation (4.33). 
The matched field source localization problems have many parameters which directly influence it's ability to detect and localize a source. A nominal set of parameters were chosen to conduct several experiments. The results of parameter estimation, i.e. DOA using the GLRT were very favorable. We conclude via the results of Table 4.1, that the error of the true DOA mean decreases as array aperature or signal power increases. In addition, the DOA variance gets smaller as we increase the array aperture, increase the signal power, model the jammer further away from the source, or some combination of these conditions.

An analysis of GLRT detectibility was conducted using the semi analytical approach to generating ROCs. We chose a nominal set of SNRs and JNRs. The background noise level was increased to twice its level between several experiments and we continued to obtain good results. We concluded that $1 \mathrm{~dB}$ increments drastically influenced GLRT's ability to detect and localize a source in high noise environments. The increase of $1 \mathrm{~dB}$ in the source power of Figure 4.8 illustrates this point. Detection and estimate unknown parameters was conducted. ROCs were simulated for several SNR and JNRs.

\subsection{Suggestions for Future Research}

This research has prompted several considerations worthy of future research. This section presents, from our perspective, those recommendations which would extend this research.

First, we suggest that future innovations in ocean acoustical modeling which results in a more accurate prediction of the replica fields be applied to this signal 
processing methodology. The $\mathrm{P} / \mathrm{E}$ solver currently is one of the most accurate and efficient models available to incorporate the ocean environmental information into MFP algorithms. Underwater acoustians continuously conduct research which result in more accurate and numerically efficient models for complex ocean environments.

Second, this investigation incorporated a deterministic, unknown signal model. The GLRT is not limited to this signal model and we hypothesize that good results may be obtained when a Gaussian signal model or random signal model is incorporated. Results from this investigation would provide additional information about the GLRT outputs under simulated data conditions before applying it to real world data.

Real world data has been collected and is conducive for matched field investigations. These data set include accurately measured geoacoustical parameters necessary for modeling and array data necessary to estimate the covariance matrix. Several data sets are available through the Office of Naval Research and Dr. Mike Porter at the New Jersey Institute of Technology. We view the major hurdles of this recommendation as a data reformatting problem and the assurance that sufficient computational power is available to implement the GLRT.

Lastly, we recommend further investigation in the GLRT maxima analysis area. Section 4.7 presented a geometrical explanation to the question, "Why does the GLRT output peak when the replica field vector matched the source field vector?". An approach to this suggestion would be to perturb the eigenvalues numerically and determine numerical bounds on these perturbations. This investigation would determine the relationship between eigenvalues and peaks of an ambiguity surface. 
We have successfully formulated matched field localization as a detection and estimation problem and demonstrated it as a viable approach to MFP. The results of this research have extended MFP contributions to include both detection of an unknown signal and estimation of the signal parameters in the presence of unknown strong directional noise. The performance analysis and representative simulation results indicate that the prescribed signal processing should provide good results as more realistic data becomes available or we choose sophisticated ocean environments for analysis. 


\section{REFERENCES}

[1] Kelly, E.J., "An adaptive detection algorithm," IEEE Transactions on Aerospace and Electronics Systems, AES-23, No. 4, pp. 115-127, November 1986.

[2] Kirsteins, I.P. and Tufts, D.W., "Rapidly adaptive nulling of interference", Lecture Notes on Control and Information Sciences, Springer-Verlag, 1990.

[3] Tufts, D.W., Kirsteins, I.P., Vaccaro, R.J., Ramalingam, C.S., and Shah A., "Improvements in signal processing for time varying multipath propagation environments," IEEE Oceans Proceedings, 1991.

[4] Tufts, D.W., Kirsteins, I.P., and Kumarsean, R., "Data-Adaptive Detection of a Weak Signal", IEEE Trans. on Aerospace and Electronic Systems, AES-19, No. 2, March 1983.

[5] Compton, Jr., R.T., Adaptive Antennas: Concepts and Performance, PrenticeHall, Englewood Cliffs, N.J. 1988.

[6] Bucker, H.P., "Use of calculated sound field and matched-field detection to locate sound source in shallow water," J. Acoust. Soc. Am. 59, pp. 368-373, February 1976.

[7] Clay, C.S., "Waveguides, Arrays, and Filters," Geophysics, Vol. XXXI, No. 3, pp. 501-505, June 1966.

[8] Hinich, M.J., "Maximum likelihood signal processing for a vertical array," J. Acoust. Soc. Am. 54, pp. $499-503$ (1973).

[9] Pekeris, C.L., "Theory of Propagation of Explosive Sound in Shallow Water," Geol. Soc. Amer. Mem. 27, pp. 1-117 (1948).

[10] Tolstoy, I., Dispersion and simple harmonic point sources in wave ducts: J. Acoust. Soc. Am., 27, pp. 897-907, 1955.

[11] Klemm, R., "Range and depth estimation by line arrays in shallow water," in Signal Processing (Reidell, Dordrecht, The Netherlands, Vol.3, pp. 333-344, October 1981.

[12] Baggeroer, A.B., Kuperman, W.A., and Schmidt, H., "Matched-field processing: Source localization in correlated noise as an optimum parameter estimation problem," J. Acoust. Soc. Am., 83, pp. 571-587, February 1988. 
[13] Schmidt, H., Baggeroer, A.B., Kuperman, W.A., and Scheer, E.K., "Environmentally tolerant beamforming for high-resolution matched field processing: Deterministic mismatch," J. Acoust. Soc. Am., 88 (4), pp. 18511862, October 1990.

[14] Ozard, J., "Matched-field processing in shallow water for range, depth, and bearing determination: results of experiment and simulation," J. Acoust. Soc. Am., 86 (2), pp. 744-453, August 1989.

[15] Feuillade, C., DelBalzo, D.R., Rowe, M.M., "Environmental mismatch in shallow-water matched-field processing: Geoacoustic parameter variability," J. Acoust. Soc. Am., 85 (6), pp. 2354-2364, June 1989.

[16] Candy, J.V. and Sullivan, E.J., "Model-based passive ranging," J. Acoust. Soc. Am., 85, pp. 2472-2480, June 1989.

[17] Hamson, R.M. and Heitmeyer, R.M., " Environmental and system effects on source localization in shallow water by matched-field processing of a vertical array," J. Acoust. Soc. Am. 86 (5), pp. 1950-1959, November 1989.

[18] Shang, E.C., Clay, C.S., and Yang, Y.Y., "Passive harmonic source ranging in waveguides using mode filter, " J. Acoust. Soc. Am., 78, pp. 172-175, November 1985.

[19] Yang, T.C., "Effectiveness of mode filtering: a comparison of matched-field and matched mode processing," J. Acoust. Soc. Am., 87 (5), pp. 2078-2084, May 1989.

[20] Zala, Z.A., and Ozard, J.M., "Matched-Field Processing in a range-dependent environment," J. Acoust. Soc. Am., 88 (2), pp. 1101-1019, August 1990.

[21] Ozard, J.M., Zakarauskas, P., and Berryman D.G., "An investigation of replica fields by a nearest neighbors algorithm for faster matched-field processing," Draft.

[22] Porter, M.B., Dicus, R., and Fizell, R.G., "Simulation of MFP in a deep-water Pacific environment," IEEE J. Oceanic Eng., Vol. OE-12, No 1, pp. 173-181, January 1987.

[23] Stergiopoulos, S., and Sullivan, E.J., "Extended towed array processor by an overlap correlator," J. Acoust. Soc. Am., 86 (1), pp. 158-171, July 1989. 
[24] Byrne, C.L., Brent, R.T., Feuillade, C.F., and DelBalzo, D.R., "A Stable dataadaptive method for matched-field array processing in acoustic waveguides," J. Acoust. Soc. Am., 87 (6), pp. 2493-2505, June 1990.

[25] Collins, M.D., and Kuperman, W.A., "Focalization: Environmental focusing and source localization," J. Acoust. Soc. Am., 90 (3), pp. 1410-1422, September 1991.

[26] Richardson, A.M., and Nolte, L.W., "A posteriori source localization in uncertain sound, deep-ocean environments," J. Acoust. Soc. Am., 89 (5), pp. 2280-2284, May 1991.

[27] Gingras, D.F., and Gerr, N.L., "Minimax robust matched-field processing," Saclant Undersea Research Centre Report, SR-201, December 1992.

[28] Van Veen, B.D., and Buckley, K.M., "Beamforming: A versatile approach to spatial filtering," IEEE ASSP Magazine, pp. 4-24, April 1988.

[29] Owsley, N.L., in Array Signal Processing, S. Haykin, ed., Prentice Hall, Englewood Cliffs, NJ 1985.

[30] Dudgeon, D.E., "Fundamentals of digital array processing, " Proceedings of the IEEE, 65, pp. 898-904, June 1977.

[31] Capon, J., "High-resolution frequency-wavenumber spectrum analysis," Proceedings of the IEEE," 57 pp. 1408-1418, August 1969.

[32] Marzetta, T.L., "A new interpretation for Capon's maximum likelihood method of frequency-wavenumber spectral estimation," IEEE Trans. on Acoustics, Speech, and Signal Processing, 31, pp. 445-449, April 1983.

[33] Burdic, W.S., Underwater Acoustic System Analysis, Prentice-Hall, Inc., Englewood Cliffs, NJ, 1984.

[34] Clay, C.S. and Medwin, H., Acoustical Oceanography: Principles and Applications, Wiley \& Sons, New York, 1977.

[35] Tolstoy, I. and Clay, C.S., Ocean Acoustics Theory and Experiment in Underwater Sound, McGraw-Hill, New York, 1966.

[36] Kreyszig, E., Advanced Engineering Mathematics, Wiley \& Sons, New York, pp. 749-759, 1962. 
[37] Collins, M.D. and Westwood, E.K. "A higher order energy-conserving parabolic equation for range-dependent ocean depth, sound speed, and density," J. Acoust. Soc. Am., 89 (3), pp. 1068-1075, March 1991.

[38] Collins, M.D., "Higher-Order Padé approximations for accurate and stable elastic parabolic equations with applications to interface wave propagation," J. Acoust. Soc. Am. 89 (3), pp. 1050-1057, March 1991.

[39] Leontovich M. and Fock, A.V., J. Phys. USSR 10, pp. 13-14, 1946.

[40] Tappert, F.D., "The Parabolic Approximation Method," in Wave Propagation and Underwater Acoustics, edited by J.B. Keller, J.S. Papadakis, Lecture Notes in Physics, Vol. 70, Springer, New York, 1977.

[41] Radder, A.C., "On The Parabolic Equation Method for Water-Wave Propagation, J. Fluid Mech. 95, Part 1, pp. 159-176, 1979.

[42] McDaniel, S.T., Parabolic approximations for underwater sound. J. Acoust. Soc. Am. 58 (6), pp. 1178-1185, December 1975.

[43] Collins, M.C., "FEPE Users Guide," Naval Ocean Research and Development Activity Note 365, October 1988.

[44] Van Trees, H.L., Detection, Estimation, and Modulation Theory - Part I, Wiley and Sons, Inc., New York, pp. 86-96, 1968.

[45] Kirsteins, I.P., "A reduced rank generalized likelihood-ratio test," TM No. 921029, Naval Undersea Warfare Center Detachment, New London, Conn., March 1992.

[46] Tufts, D.W., Kirsteins, I.P, Vaccaro, R.J., Ramalignam, C.S., and Shah, A., " Improvements in Signal Processing for Time-Varying Multipath Propagation Environments", pp. 586- 593, IEEE Oceans 1991 Proceedings,

[47] Brooks, L.W. and Reed, I.S., "Equivalence of Likelihood Ratio Processor, The Maximum Signal-to-Noise Ratio Filter, and the Wiener Filter," IEEE Trans. Aerospace and Electronic Systems, pp. 690-692, September. 1972,

[48] Kirsteins, I.P., "Analysis of Reduced Rank Interference Cancellation", Ph.D Thesis, University of Rhode Island, 1991.

[49] Christensen, R., "Plane Answers to Complex Questions The Theory of Linear Models," Springer-Verlag, pp. 1-9,14-25, New York, 1987. 
[50] Scharf, L.L., "Statistical Signal Processing - Detection, Estimation, and Time Series Analysis," Addison-Wesley, Reading MA., 1991.

[51] Stewart, G.W. and Sun, J., "Matrix Perturbation Theory," Academic Press, Inc., p. 34, New York, 1990,

[52] Anderson, T.W., "An Introduction to Multivariate Statistical Analysis," 2nd Edition, pp. 60-64 and 287-289, John Wiley and Sons, New York, 1984.

[53] Strang, G., "Linear Algebra and Its Applications," Academic Press, Inc., pp. 103-110. New York, 1980.

[54] Golub, G.H. and Van Loan, C.F., "Matrix Computations," The Johns Hopkins University Press, pp. 70-75, Baltimore, 1989.

[55] Collins, M.D., "A split-step Padé solution for the parabolic equation method," J. Acoust. Soc. Am. 93 (4), pp. 1736-1741, April 1993. 


\section{APPENDIX A The Parabolic Equation Derivation}

We derive the three dimensional reduced wave equation for the cylindrically symmetric case given by equation (8) of reference [24]. This derivation is the mathematical basis for the $\mathrm{P} / \mathrm{E}$ Solver algorithm used in this investigation. It is the mathematical formulation for the complex pressure concerns waves propagation in the positive $r$ direction and neglects the influence of the waves in the opposite direction. We start with factoring the $\mathbf{Q}$ operator, neglect backscattered waves and apply the Padé approximates. The PE method is based on an approximation of the square-root function which arises when the wave equation is factored into incoming and outgoing solutions. Specifically, we follow the split-step Padé approximation which handles wide propagation angles and large sound-speed variations more accurately than Fourier solutions [55].

Consider the nonhomogeneous wave equation

$$
\rho \partial \nabla \cdot\left[\left(\frac{1}{\rho} \nabla P\right)\right]+K^{2} P=-4 \pi \delta\left(x-x_{s}\right), \text { where }
$$

$\mathrm{P}$ is the acoustic pressure satisfying the boundary conditions,

$\mathrm{K}=\mathrm{k}(1+\mathrm{i} \eta \beta)$ is the complex wavenumber,

$\mathrm{k}=\omega / \mathrm{c}$

$\mathrm{c}$ is the sound speed,

$\beta$ is the attenuation constant $(\mathrm{dB} / \lambda)$,

$\mathrm{x}$ is the is the Cartesian position vector,

$\mathrm{x}_{\mathbf{s}}$ is the source location,

$\eta=\left(40 \pi \log _{10} \mathrm{e}\right)^{-1}$, and

$\rho$ is the water density.

We use the well known vector identity, 


$$
\nabla \cdot(\beta A)=\beta \nabla \cdot A+A \cdot \nabla \beta
$$

where

$$
\beta=\frac{1}{\rho} \quad \text { and } \quad A=\nabla \beta .
$$

Equation (A.1) then becomes

$$
\begin{aligned}
& \nabla \cdot\left(\frac{1}{\rho} \nabla P\right)=\frac{1}{\rho}(\nabla \cdot \nabla P)+\nabla P \cdot \nabla\left(\frac{1}{\rho}\right) \\
& \nabla \cdot\left(\frac{1}{\rho} \nabla P\right)=\frac{1}{\rho} \nabla^{2} \cdot \nabla P+\nabla P \cdot \nabla\left(\frac{1}{\rho}\right) \\
& \rho \nabla \cdot\left(\frac{1}{\rho} \nabla P\right)=\nabla^{2} \cdot \nabla P+\rho \nabla P \cdot\left(\frac{1}{\rho}\right) .
\end{aligned}
$$

In cylindrical coordinates we define $\nabla^{2}(\cdot)$ and $\nabla(\cdot)$ as:

$$
\nabla^{2} \Psi \equiv \frac{1}{r} \frac{\partial}{\partial r}\left(r \partial \frac{\Psi}{\partial r}\right)+\frac{\partial^{2} \Psi}{\partial z^{2}}+\frac{\partial^{2} \Psi}{\partial \Phi^{2}}
$$

and 


$$
\nabla \Psi \equiv r \frac{\partial \Psi}{\partial r}+z \frac{\partial \Psi}{\partial z}+\phi \frac{\partial \Psi}{\partial \phi}
$$

Our problem geometry dictates an independence of $\phi$. We apply equations (A.7) and (A.8) to equation (A.6) to obtain:

$$
\begin{gathered}
\rho \nabla \cdot\left(\frac{1}{\rho} \nabla P\right)=\frac{1}{r} \frac{\partial}{\partial r}\left(r \frac{\partial P}{\partial r}+\frac{\partial^{2} P}{\partial z^{2}}\right)+\rho r\left(r \frac{\partial P}{\partial r}+z \frac{\partial P}{\partial z}\right) \cdot\left(r \frac{\partial\left(\frac{1}{\rho}\right)}{\partial r}+z \frac{\partial\left(\frac{1}{\rho}\right)}{\partial z}\right) \\
\rho \nabla \cdot\left(\frac{1}{\rho} \nabla P\right)=\frac{\partial^{2} P}{\partial r^{2}}+\frac{1}{r} \frac{\partial P}{\partial r}+\frac{\partial^{2} P}{\partial z^{2}}+\rho \frac{\partial P}{\partial r} \frac{\partial\left(\frac{1}{\rho}\right)}{\partial r}+\rho \frac{\partial P}{\partial z} \frac{\partial\left(\frac{1}{r}\right)}{\partial z} .
\end{gathered}
$$

Collecting common terms we get equation (A.10) we get:

$$
=\frac{\partial^{2} P}{\partial r^{2}}+\rho \frac{\partial P}{\partial r} \frac{\partial\left(\frac{1}{\rho}\right)}{\partial r}+\frac{\partial^{2} P}{\partial z^{2}}+\rho \frac{\partial P}{\partial z} \frac{\partial\left(\frac{1}{\rho}\right)}{\partial z}+\frac{1}{r} \frac{\partial P}{\partial r}+\delta\left(x-x_{s}\right)=\frac{\delta(r) \delta\left(z-z_{o}\right)}{2 \pi r}
$$

Proceeding with simplification we obtain equation (2) of reference [24] in equation (A.11). 


$$
\begin{aligned}
& =\rho \frac{\partial}{\partial r}\left(\frac{1}{\rho} \frac{\partial P}{\partial r}\right)+\rho \frac{\partial}{\partial z}\left(\frac{1}{\rho} \frac{\partial P}{\partial z}\right)+\frac{1}{r} \frac{\partial P}{\partial r}+\delta\left(x-x_{s}\right)=\frac{\delta(r) \delta\left(z-z_{o}\right)}{2 \pi r} \\
& =\rho \frac{\partial}{\partial r}\left(\frac{1}{\rho} \frac{\partial P}{\partial r}\right)+\frac{1}{r} \frac{\partial P}{\partial r}+\rho \frac{\partial}{\partial z}\left(\frac{1}{\rho} \frac{\partial P}{\partial z}\right)+K^{2} P=-4 \pi \frac{\delta(r) \delta\left(z-z_{o}\right)}{2 \pi r} \\
& =\rho \frac{\partial}{\partial r}\left(\frac{1}{\rho} \frac{\partial P}{\partial r}\right)+\frac{1}{r} \frac{\partial P}{\partial r}+\rho \frac{\partial}{\partial z}\left(\frac{1}{\rho} \frac{\partial P}{\partial z}\right)+K^{2} P=-\frac{2}{r} \delta(r) \delta\left(z-z_{0}\right) .
\end{aligned}
$$

Next we define $p=r^{1 / 2} P \rightarrow P=r^{-1 / 2} p$ to remove spherical spreading and proceed to define each term of equation (A.14) as:

$$
\begin{aligned}
& \frac{\partial^{2} P}{\partial r^{2}}=\left(-\frac{1}{2}\right)\left(\frac{1}{r^{\frac{3}{2}}}\right) P+\left(\frac{1}{r^{\frac{1}{2}}}\right) \frac{\partial P}{\partial r} \\
& \frac{\partial^{2} P}{\partial r^{2}}=\left(\frac{3}{4}\right)\left(\frac{1}{r^{\frac{5}{2}}}\right) P-\left(\frac{1}{r^{\frac{3}{2}}}\right) \frac{\partial P}{\partial r}+\left(\frac{1}{r^{\frac{1}{2}}}\right) \frac{\partial^{2} P}{\partial r^{2}} \\
& \frac{\partial P}{\partial z}=\left(\frac{1}{r^{\frac{1}{2}}}\right) \frac{\partial P}{\partial z} \\
& \frac{\partial^{2} P}{\partial z}=\left(\frac{1}{r^{\frac{1}{2}}}\right) \frac{\partial^{2} P}{\partial z^{2}} .
\end{aligned}
$$


Substituting the definitions of equation (A.15) into equation (A.14) we get:

$$
\begin{gathered}
=\left(\frac{3}{4}\right)\left(\frac{1}{r^{5 / 2}}\right) P-\left(\frac{1}{r^{3 / 2}}\right) \frac{\partial P}{\partial r}+\frac{1}{r^{1 / 2}} \frac{\partial^{2} P}{\partial r^{2}}-(1 / 2) \rho \frac{1}{r^{3 / 2}} P\left(\frac{\partial\left(\frac{1}{\rho}\right)}{\partial r}\right) \\
+\left(\frac{1}{r^{1 / 2}}\right)(\rho)\left(\frac{\partial\left(\frac{1}{\rho}\right)}{\partial r}\right)+\frac{1}{r^{1 / 2}} \frac{\partial^{2} P}{\partial z^{2}}+\left(\frac{1}{r^{1 / 2}}\right)(\rho)\left(\frac{\partial P}{\partial z}\right)\left(\frac{\partial\left(\frac{1}{\rho}\right)}{\partial z}\right) \\
-\left(\frac{1}{2}\right)\left(\frac{1}{r^{5 / 2}}\right) P+\left(\frac{1}{r^{3 / 2}}\right)\left(\frac{\partial P}{\partial r}\right)+\frac{k^{2} p}{r^{1 / 2}}=\frac{-2}{r} \delta(r) \delta\left(z-z_{0}\right)
\end{gathered}
$$

which simplifies to:

$$
\begin{aligned}
& =\left(\frac{1}{4}\right)\left(\frac{1}{r^{5 / 2}}\right) P+\left(\frac{1}{r^{\frac{1}{2}}}\right) \frac{\partial^{2} P}{\partial r^{2}}-\left(\frac{1}{2}\right) \rho\left(\frac{1}{r^{3 / 2}}\right) P\left(\frac{\left.\partial\left(\frac{1}{\rho}\right)\right)+\left(\frac{1}{\partial r}\right)(\rho)\left(\frac{\partial P}{\partial r}\right)\left(\frac{\partial\left(\frac{1}{\rho}\right)}{\partial r}\right)}{\left.r^{1 / 2}\right)}\right. \\
& +\left(\frac{1}{r^{1 / 2}}\right) \frac{\partial^{2} P}{\partial z^{2}}+\left(\frac{1}{r^{1 / 2}}\right)(\rho)\left(\frac{\partial P}{\partial z}\right)\left(\frac{\partial\left(\frac{1}{\rho}\right)}{\partial z}\right)+\frac{k^{2} p}{r^{1 / 2}}=\frac{-2}{r} \delta(r) \delta\left(z-z_{o}\right) .
\end{aligned}
$$


Multiply by equation (A.17) by $r^{1 / 2}$ we get:

$$
\begin{aligned}
& =\left(\frac{1}{4 r^{2}}\right) P-\rho \frac{1}{2 r} P\left(\frac{\partial\left(\frac{1}{\rho}\right)}{\partial r}\right)+\frac{\partial^{2} P}{\partial z^{2}}+(\rho) \frac{\partial P}{\partial z}\left(\frac{\partial\left(\frac{1}{\rho}\right)}{\partial z}\right)+\frac{\partial^{2} P}{\partial r^{2}} \\
& +(\rho)\left(\frac{\partial P}{\partial r}\right)\left(\frac{\partial\left(\frac{1}{\rho}\right)}{\partial r}\right)+k^{2} p=\frac{-2}{r^{1 / 2}} \delta(r) \delta\left(z-z_{o}\right) .
\end{aligned}
$$

In the far field $r$ is large, $1 / r^{2}$ and $1 / r$ of equation (A.18) tend to zero, therefore

$$
\begin{aligned}
& =\frac{\partial^{2} P}{\partial z^{2}}+(\rho) \frac{\partial P}{\partial z}\left(\frac{\partial\left(\frac{1}{\rho}\right)}{\partial z}\right)+\frac{\partial^{2} P}{\partial r^{2}} \\
& +(\rho)\left(\frac{\partial P}{\partial r}\right)\left(\frac{\partial\left(\frac{1}{\rho}\right)}{\partial r}\right)+k^{2} p=\frac{-2}{r^{1 / 2}} \delta(r) \delta\left(z-z_{o}\right) .
\end{aligned}
$$


We apply vector calculus to equation (A.19) in order to reformulate it as:

$$
\rho \frac{\partial}{\partial r}\left(\frac{1}{\rho} \frac{\partial r}{\partial r}\right)+\rho \frac{\partial}{\partial z}\left(\frac{1}{\rho} \frac{\partial p}{\partial z}\right)+k^{2} p=0
$$

which is equation (3) of reference [24]. Next we define an operator $\mathbf{Q}$ as:

$$
\boldsymbol{Q}=\frac{\partial}{\partial \boldsymbol{r}}[31]
$$

and after additional manipulations for range independent environments equation (A.21) can be written as:

$$
\frac{\partial p}{\partial r}=i k_{o} \sqrt{1+k_{o}^{-2}\left(\rho \frac{\partial}{\partial z} \frac{1}{\rho} \frac{\partial}{\partial z}+K^{2}-k_{o}^{2}\right) p}
$$

where $k_{o}=\omega / c_{o}$ is the reference wave number and $c_{o}$ is the reference sound speed.

The acoustic parameters $c, \rho$, and $\beta$ depend only on depth and not range in equation (A.22). We continue the derivation of the PE based upon an approximation to the function $f(x)=\sqrt{1+x}$, where $f(x)$ is replaced by the Padé approximations for the square root function [55]. The following higher-order PE is obtained:

$$
\frac{\partial u}{\partial r}=i k_{o} \sum_{j=1}^{n} \frac{a_{j, n}\left(\rho \frac{\partial}{\partial \sigma} \frac{1}{\rho} \frac{\partial}{\partial \sigma}+K^{2}-k_{o}^{2}\right)}{k_{o}^{2}+b_{j, n}\left(\rho \frac{\partial}{\partial \sigma} \frac{1}{\rho} \frac{\partial}{\partial \sigma}+K^{2}-k_{o}^{2}\right)} p
$$


where $\mathrm{a}_{\mathrm{j}, \mathrm{n}}$ and $\mathrm{b}_{\mathrm{j}, \mathrm{n}}$ are the Padé coefficients. The Padé coefficients are defined as:

$$
\begin{aligned}
& a_{j, n}=\frac{2}{2 n+1} \sin ^{2} \frac{j \pi}{2 n+1} \quad \text { and } \\
& b_{j, n}=\cos ^{2} \frac{j \pi}{2 n+1} .
\end{aligned}
$$

Equation (A.23) is now applied to range-dependent problems by allowing $K, \rho$, and $\alpha$ to depend on range. Equation (A.23) requires the solution a numerical approach. This solution is implemented in the P/E Solver software code (Appendix B) and is used to model sound propagation in range-dependent as well as range independent environments. 


\section{APPENDIX B Parabolic Equation Solver Code}

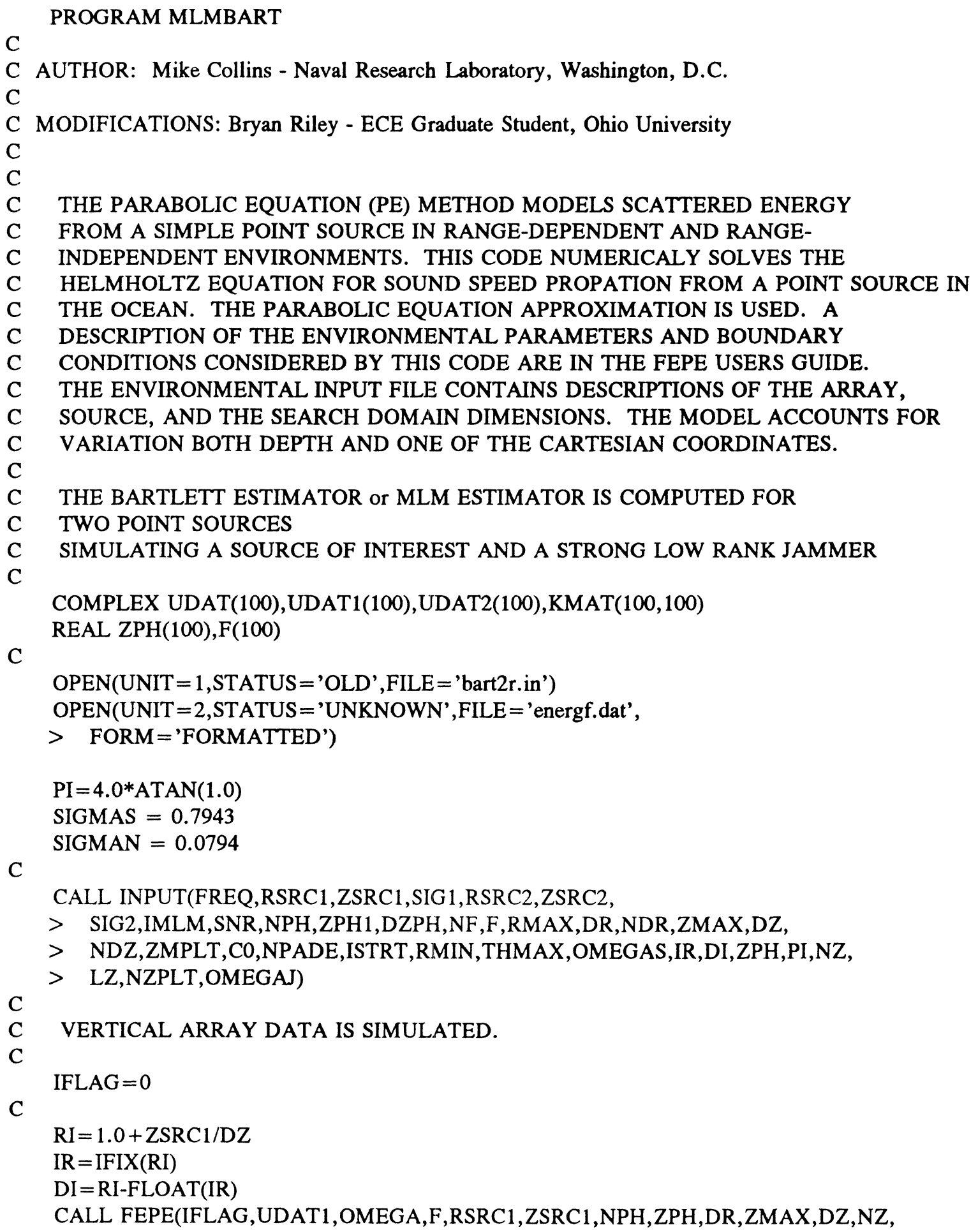


C

$>$ C0,NPADE,ISTRT,RMIN,THMAX,IR,DI,LZ,NZPLT,NDZ,NDR,

$$
>\text { KMAT,IMLM) }
$$

$\mathrm{RI}=1.0+\mathrm{ZSRC} 2 / \mathrm{DZ}$

$\mathrm{IR}=\mathrm{IFIX}(\mathrm{RI})$

$\mathrm{DI}=\mathrm{RI}-\mathrm{FLOAT}(\mathrm{IR})$

CALL FEPE(IFLAG,UDAT2,OMEGA,F,RSRC2,ZSRC2,NPH,ZPH,DR,ZMAX,DZ,NZ,

$>$ C0,NPADE,ISTRT,RMIN,THMAX,IR,DI,LZ,NZPLT,NDZ,NDR,

$\mathrm{C}$

$>$ KMAT,IMLM)

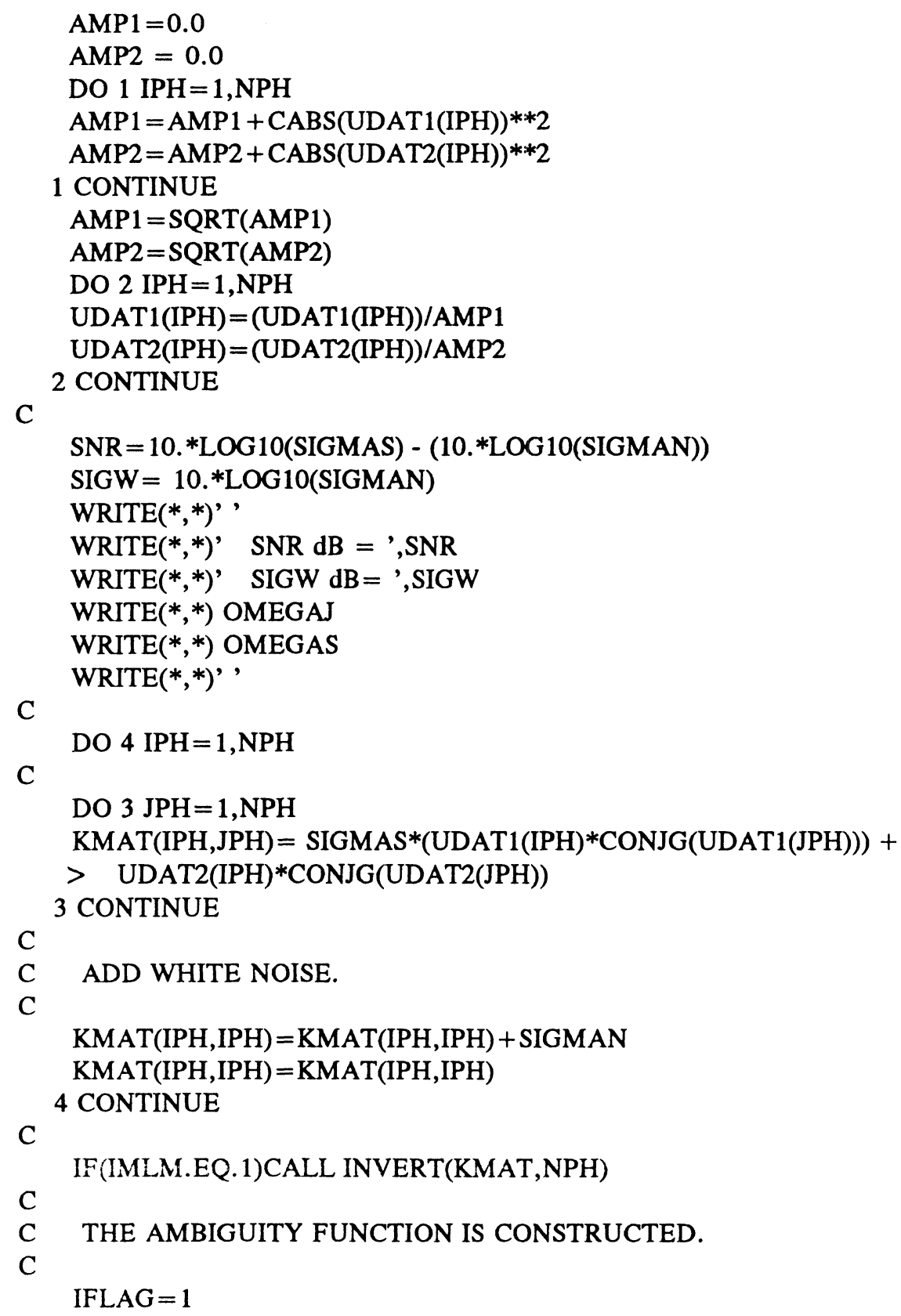


CALL FEPE(IFLAG,UDAT,OMEGA,F,RMAX,ZSRC,NPH,ZPH,DR,ZMAX,DZ,NZ,

C

$>$ C0,NPADE,ISTRT,RMIN,THMAX,IR,DI,LZ,NZPLT,NDZ,NDR,

$>$ KMAT,IMLM)

C

STOP

END

$\mathrm{C}$

C INPUT DATA.

C

SUBROUTINE INPUT(FREQ,RSRC1,ZSRC1,SIG1,RSRC2,ZSRC2,

$>$ SIG2,IMLM,SNR,NPH,ZPH1,DZPH,NF,F,RMAX,DR,NDR,ZMAX,DZ,

$>$ NDZ,ZMPLT,CO,NPADE,ISTRT,RMIN,THMAX,OMEGA,IR,DI,ZPH,PI,

$>$ NZ,LZ,NZPLT)

REAL F(100),ZPH(100)

C

C

C $\operatorname{READ}(1, *)$

READ $(1, *)$ FREQ

READ $(1, *)$ RSRC1,ZSRC1,SIG1

$\operatorname{READ}(1, *)$ RSRC2,ZSRC2,SIG2

READ $(1, *)$ IMLM,SNR

C

READ $(1, *)$ NPH,ZPH1,DZPH

$\operatorname{READ}(1, *) \mathrm{NF}$

DO $1 \mathrm{~J}=1, \mathrm{NF}$

$\operatorname{READ}(1, *) \mathrm{F}(\mathrm{J})$

1 CONTINUE

C

READ $(1, *)$ RMAX,DR,NDR

$\operatorname{READ}(1, *) Z M A X, D Z, N D Z, Z M P L T$

$\operatorname{READ}(1, *)$ C0,NPADE

C

READ $(1, *)$ ISTRT, RMIN,THMAX

WRITE $(*, *)$ NPADE

WRITE $(*, *)$ ZSRC2

WRITE $(*, *)$ RSRC1

THMAX $=$ THMAX $* \mathrm{PI} / 180.0$

OMEGAS $=2.0 *$ PI*FREQ

$\mathrm{NZ}=\mathrm{ZMAX} / \mathrm{DZ}-0.5$

NZPLT $=$ ZMPLT/DZ-0.5

C

$\mathrm{OMEGAJ}=2.0 * \mathrm{PI} *(\mathrm{FREQ} / 2 .+7$. $)$

$\mathrm{RI}=1.0+\mathrm{ZSRC} 1 / \mathrm{DZ}$

IR $=$ IFIX(RI)

$\mathrm{DI}=\mathrm{RI}-\mathrm{FLOAT}(\mathrm{IR})$

C

$\mathrm{ZPH}(1)=\mathrm{ZPH} 1$

DO 2 IPH $=2, N P H$

$\mathrm{ZPH}(\mathrm{IPH})=\mathrm{ZPH}(\mathrm{IPH}-1)+\mathrm{DZPH}$

2 CONTINUE

C 


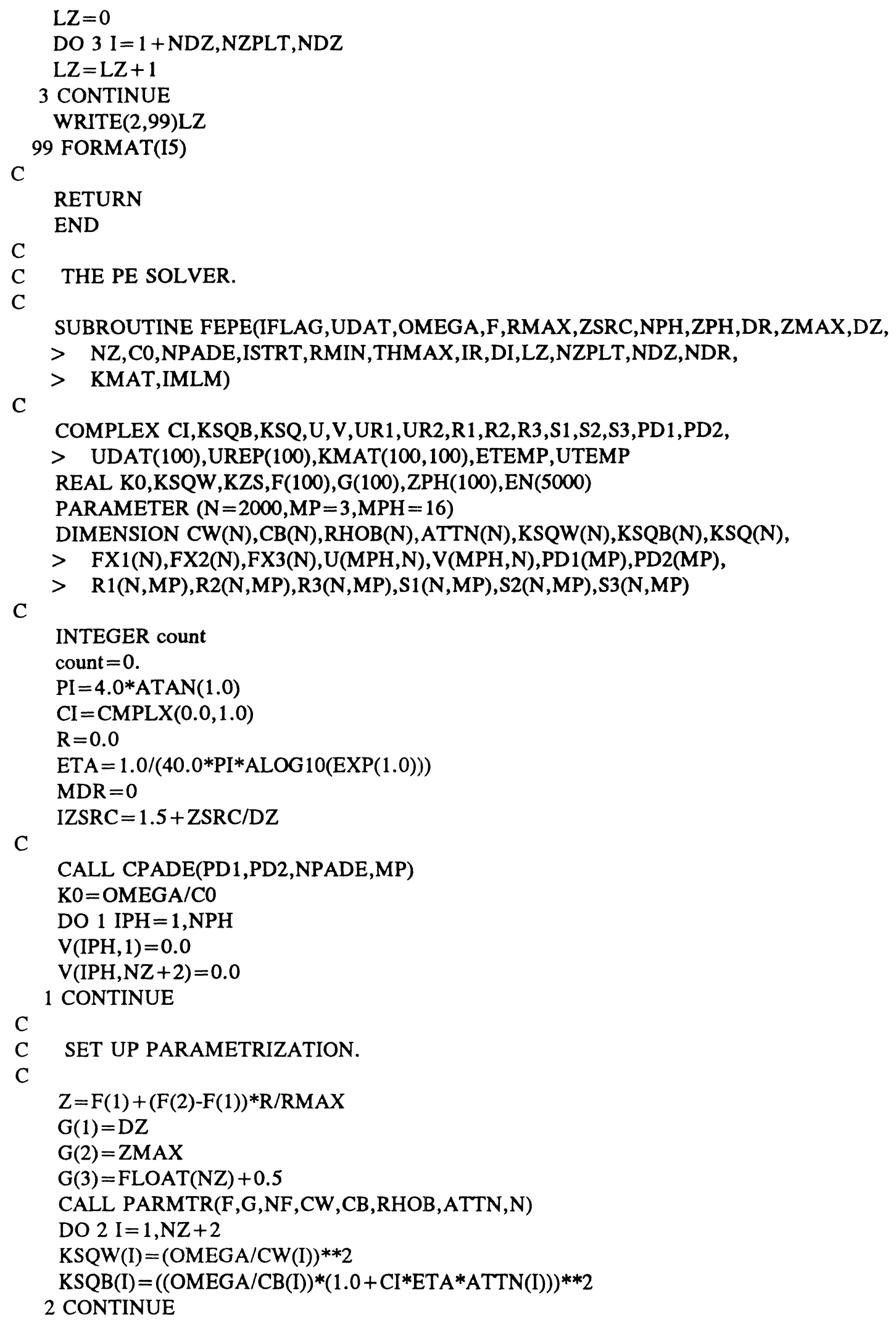




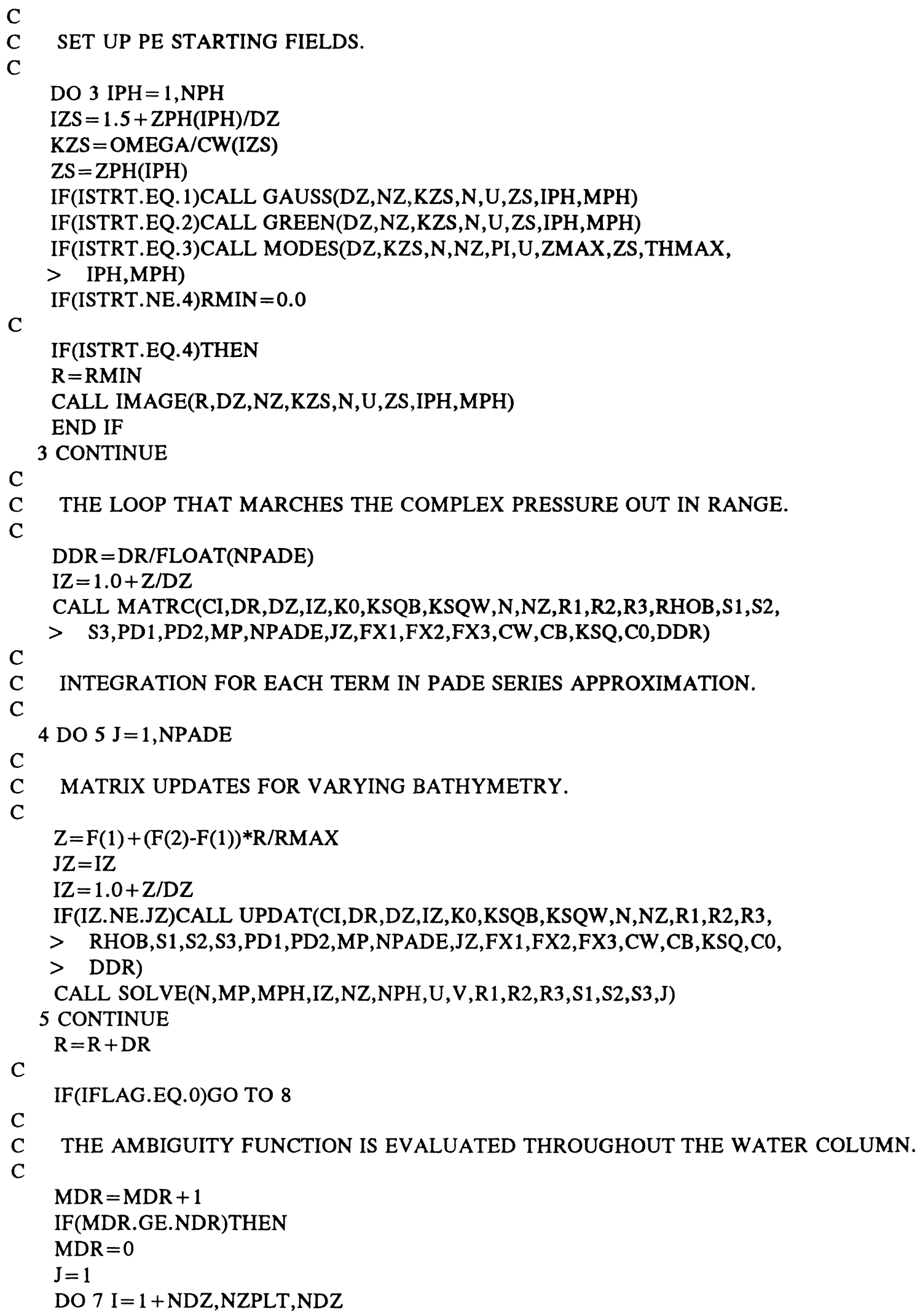




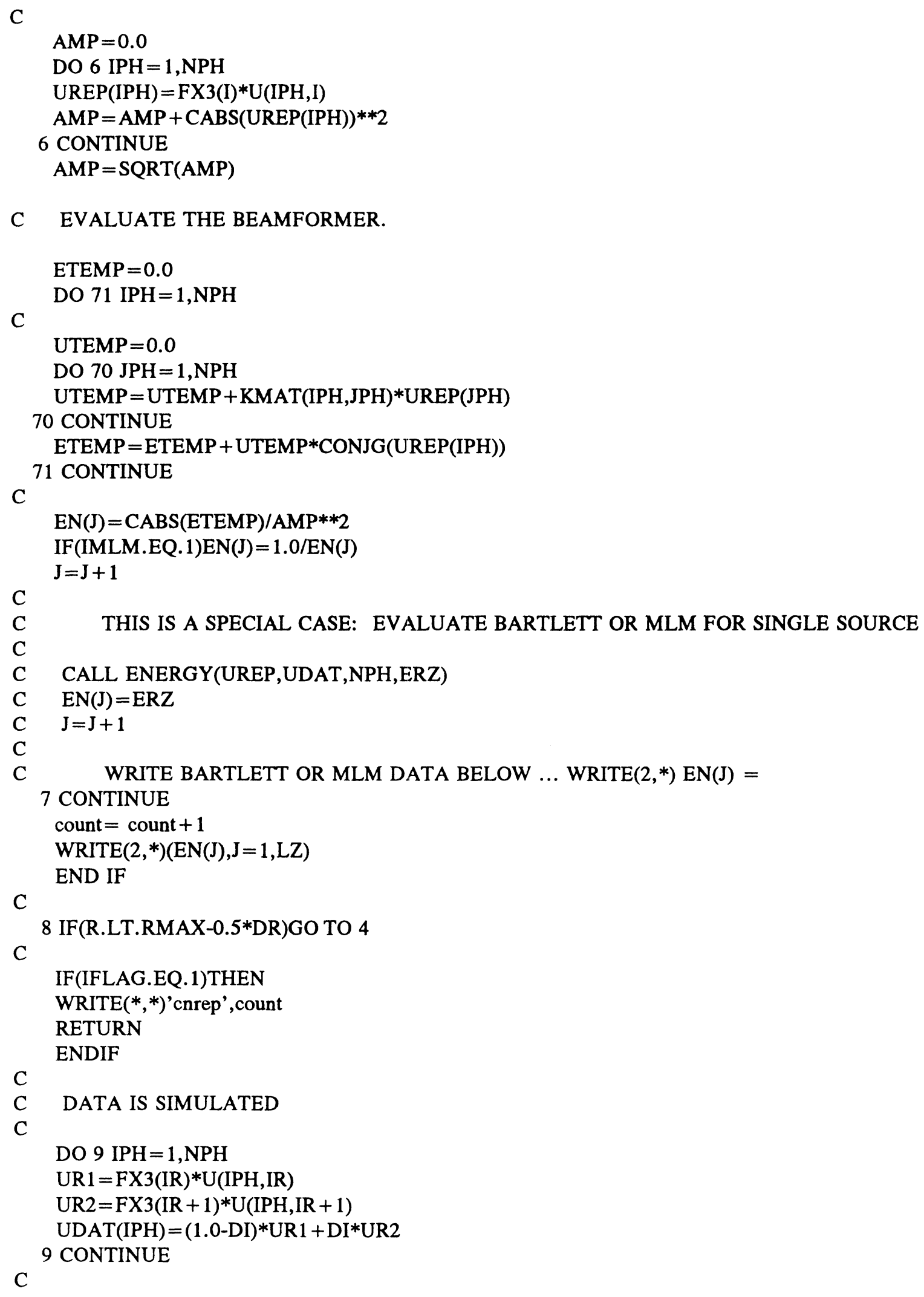




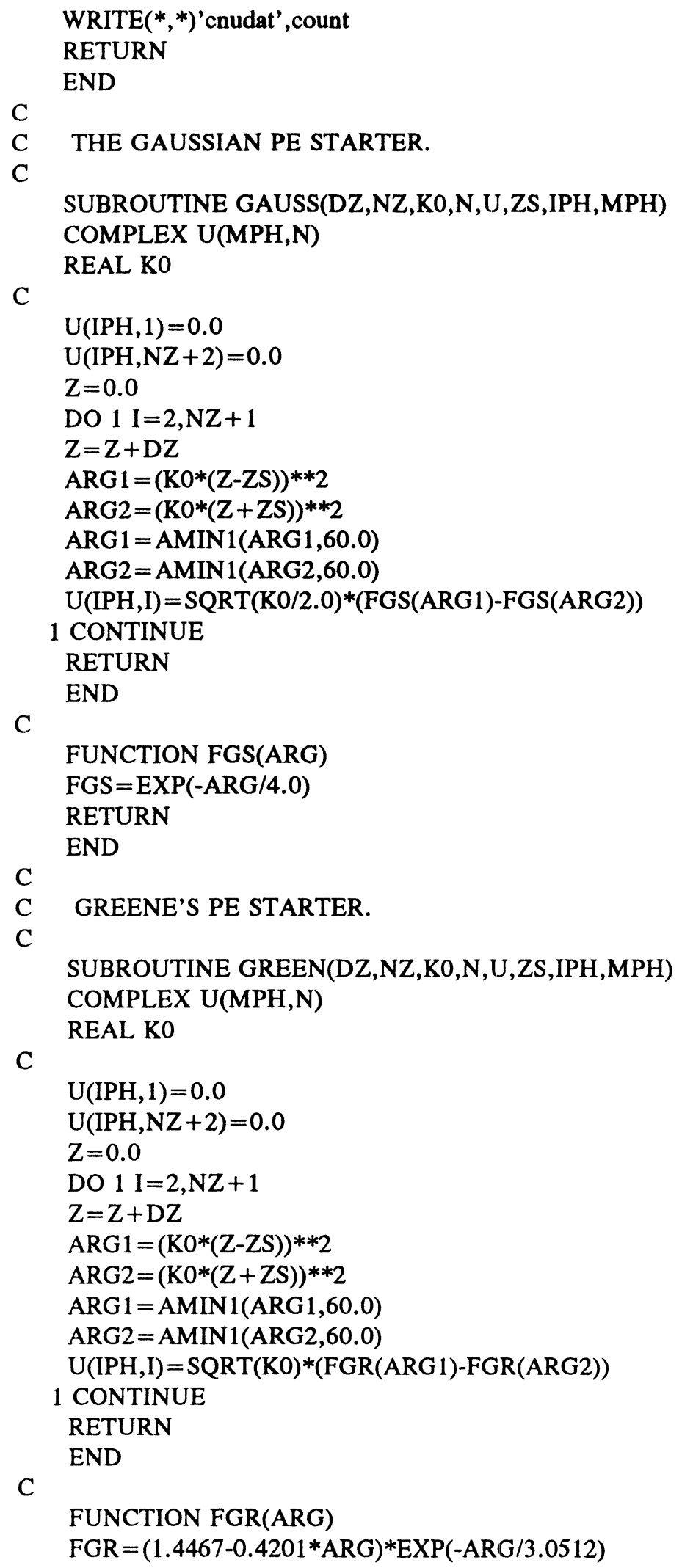




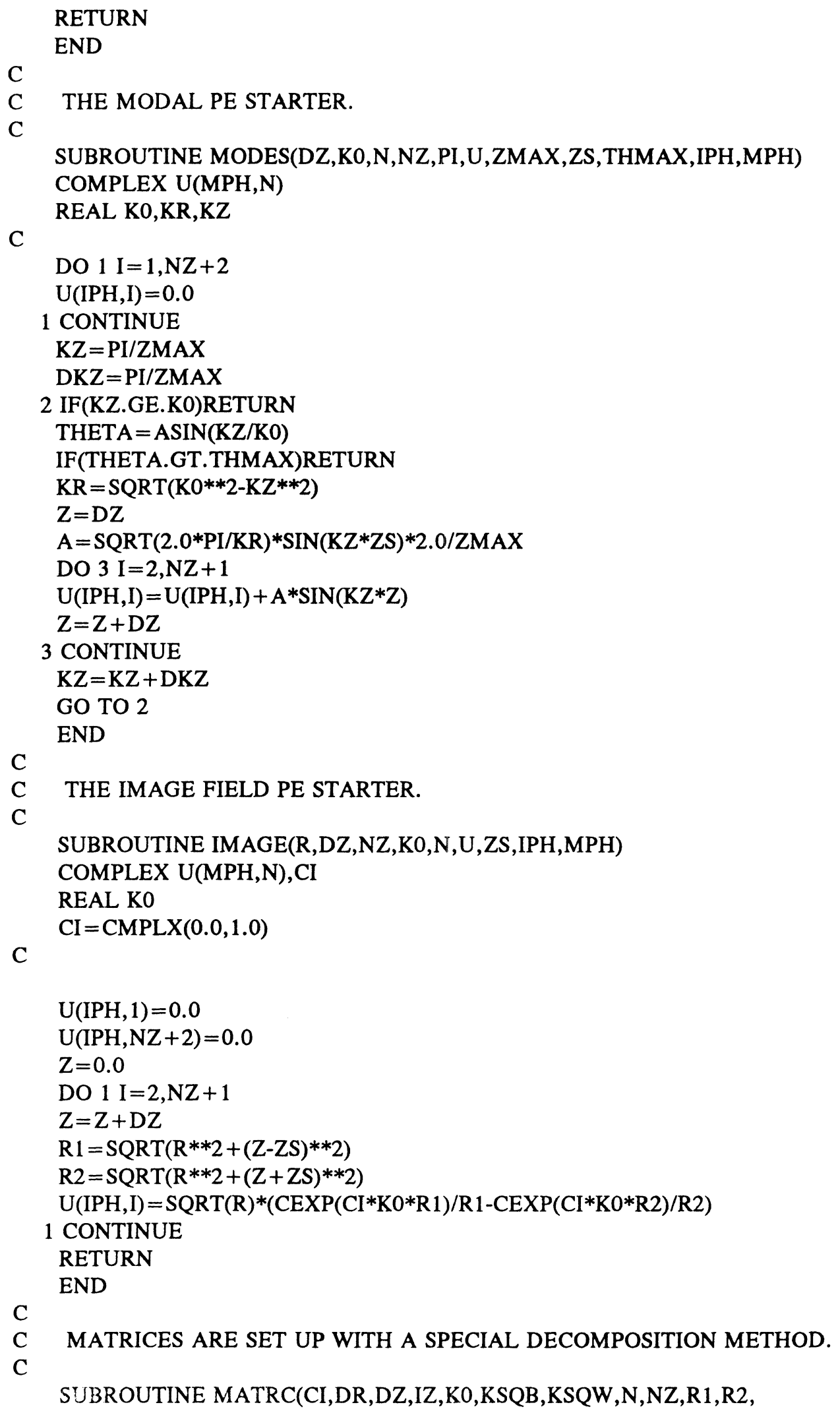


$>$ R3,RHOB,S1,S2,S3,PD1,PD2,MP,NPADE,JZ,FX1,FX2, $>\mathrm{FX3}, \mathrm{CW}, \mathrm{CB}, \mathrm{KSQ}, \mathrm{CO}, \mathrm{DDR})$ COMPLEX CI,D1,D2,D3,E1,E2,E3,F1,F2,F3,KSQB(N),KSQ(N),

$>\mathrm{R} 1(\mathrm{~N}, \mathrm{MP}), \mathrm{R} 2(\mathrm{~N}, \mathrm{MP}), \mathrm{R} 3(\mathrm{~N}, \mathrm{MP}), \mathrm{S} 1(\mathrm{~N}, \mathrm{MP}), \mathrm{S} 2(\mathrm{~N}, \mathrm{MP}), \mathrm{S} 3(\mathrm{~N}, \mathrm{MP})$,

$>$ PD1(MP),PD2(MP),PD3,PD4,KB

REAL K0,KSQW(N), RHOB(N),CW(N), CB(N),FX1(N),FX2(N),FX3(N)

C

C THE COEFFICIENTS FOR THE RADIATION BOUNDARY CONDITION.

$\mathrm{C}$

$\mathrm{PD} 3=0.2165063 * \mathrm{CI}$

$\mathrm{PD} 4=0.6856034 * \mathrm{CI}$

$\mathrm{KB}=\mathrm{CSQRT}(\mathrm{KSQB}(\mathrm{NZ}+2))$

C

$\mathrm{A} 1=1.0 / 6.0$

$\mathrm{A} 2=2.0 / 3.0$

$\mathrm{A} 3=1.0 / 6.0$

C

DO $3 \mathrm{~J}=1$, NPADE

$\mathrm{E} 1=\mathrm{K} 0 * * 2 * \mathrm{~A} 1+\mathrm{PD} 2(\mathrm{~J}) * \mathrm{D} 1$

$\mathrm{E} 2=\mathrm{K} 0 * * 2 * \mathrm{~A} 2+\mathrm{PD} 2(\mathrm{~J}) * \mathrm{D} 2$

$\mathrm{E} 3=\mathrm{K} 0 * * 2 * \mathrm{~A} 3+\mathrm{PD} 2(\mathrm{~J}) * \mathrm{D} 3$

$\mathrm{F} 1=\mathrm{PD} 1(\mathrm{~J}) * \mathrm{CI} * \mathrm{~K} 0 * \mathrm{D} 1$

$\mathrm{F} 2=\mathrm{PD} 1(\mathrm{~J}) * \mathrm{CI} * \mathrm{~K} 0 * \mathrm{D} 2$

$\mathrm{F} 3=\mathrm{PD} 1(\mathrm{~J}) * \mathrm{CI} * \mathrm{~K} 0 * \mathrm{D} 3$

$\mathrm{R} 1(\mathrm{I}, \mathrm{J})=\mathrm{E} 1-0.5 * \mathrm{DR} * \mathrm{~F} 1$

$\mathrm{R} 2(\mathrm{I}, \mathrm{J})=\mathrm{E} 2-0.5 * \mathrm{DR} * \mathrm{~F} 2$

$\mathrm{R} 3(\mathrm{I}, \mathrm{J})=\mathrm{E} 3-0.5 * \mathrm{DR} * \mathrm{~F} 3$

$\mathrm{S} 1(\mathrm{I}, \mathrm{J})=\mathrm{E} 1+0.5 * \mathrm{DR} * \mathrm{~F} 1$ 


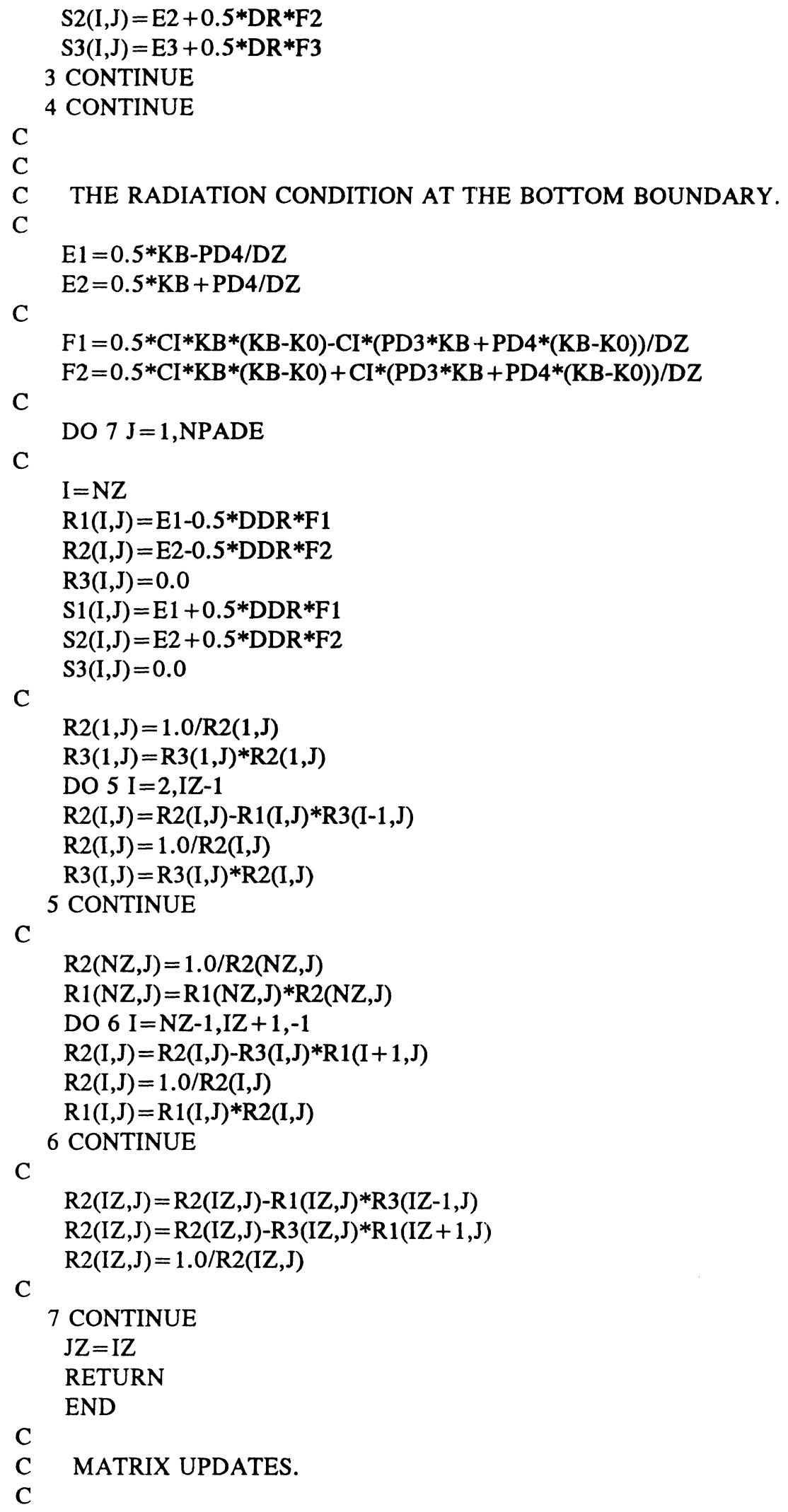


SUBROUTINE UPDAT(CI,DR,DZ,IZ,K0,KSQB,KSQW,N,NZ,R1,R2,R3, $>$ RHOB,S1,S2,S3,PD1,PD2,MP,NPADE,JZ,FX1,FX2,FX3,CW,CB, $>\mathrm{KSQ}, \mathrm{C} 0, \mathrm{DDR})$ COMPLEX CI,D1,D2,D3,E1,E2,E3,F1,F2,F3,KSQB(N),KSQ(N), $>\quad \mathrm{R} 1(\mathrm{~N}, \mathrm{MP}), \mathrm{R} 2(\mathrm{~N}, \mathrm{MP}), \mathrm{R} 3(\mathrm{~N}, \mathrm{MP}), \mathrm{S} 1(\mathrm{~N}, \mathrm{MP}), \mathrm{S} 2(\mathrm{~N}, \mathrm{MP}), \mathrm{S} 3(\mathrm{~N}, \mathrm{MP})$, $>$ PD1(MP),PD2(MP)

C REAL K0,KSQW(N), RHOB(N),CW(N),CB(N),FX1(N),FX2(N),FX3(N)

$\mathrm{A} 1=1.0 / 6.0$
$\mathrm{~A} 2=2.0 / 3.0$
$\mathrm{~A} 3=1.0 / 6.0$

C

IF(IZ.GT.JZ)THEN

$\mathrm{I} 1=\mathrm{JZ}-1$

$\mathrm{I} 2=\mathrm{IZ}$

DO $1 \mathrm{I}=\mathrm{JZ}+1, \mathrm{IZ}$

$\mathrm{FX} 1(\mathrm{I})=1.0 / \mathrm{SQRT}(\mathrm{CW}(\mathrm{I}) / \mathrm{CO})$

$\mathrm{FX} 2(\mathrm{I})=1.0$

$\mathrm{FX3}(\mathrm{I})=\mathrm{SQRT}(\mathrm{CW}(\mathrm{I}) / \mathrm{C} 0)$

$\mathrm{KSQ}(\mathrm{I})=\mathrm{KSQW}(\mathrm{I})-\mathrm{KO} * * 2$

C

1 CONTINUE

$$
\begin{aligned}
& \text { ELSE } \\
& \mathrm{I} 1=\mathrm{IZ}-1 \\
& \mathrm{I} 2=\mathrm{JZ} \\
& \mathrm{DO} 2 \mathrm{I}=\mathrm{IZ}+1, \mathrm{JZ} \\
& \mathrm{FX} 1(\mathrm{I})=\mathrm{RHOB}(\mathrm{I}) / \mathrm{SQRT}(\mathrm{RHOB}(\mathrm{I}) * \mathrm{CB}(\mathrm{I}) / \mathrm{C} 0) \\
& \mathrm{FX} 2(\mathrm{I})=1.0 / \mathrm{RHOB}(\mathrm{I}) \\
& \mathrm{FX} 3(\mathrm{I})=\mathrm{SQRT}(\mathrm{RHOB}(\mathrm{I}) * \mathrm{CB}(\mathrm{I}) / \mathrm{C} 0) \\
& \mathrm{KSQ}(\mathrm{I}=\mathrm{KSQB}(\mathrm{I})-\mathrm{K} 0 * * 2 \\
& 2 \mathrm{CONTINUE} \\
& \mathrm{END} \mathrm{IF} \\
& \mathrm{DO} 4 \mathrm{I}=\mathrm{I} 1, \mathrm{I} 2 \\
& \mathrm{C} 1=(0.5 / \mathrm{DZ} * * 2) * \mathrm{FX} 1(\mathrm{I}+1) *(\mathrm{FX} 2(\mathrm{I})+\mathrm{FX} 2(\mathrm{I}+1)) * \mathrm{FX} 3(\mathrm{I}) \\
& \mathrm{C} 2=-(0.5 / \mathrm{DZ} * * 2) * \mathrm{FX} 1(\mathrm{I}+1) *(\mathrm{FX} 2(\mathrm{I})+2.0 * \mathrm{FX} 2(\mathrm{I}+1)+ \\
& >\quad \mathrm{FX} 2(\mathrm{I}+2)) * \mathrm{FX} 3(\mathrm{I}+1) \\
& \mathrm{C} 3=(0.5 / \mathrm{DZ} * * 2) * \mathrm{FX} 1(\mathrm{I}+1) *(\mathrm{FX} 2(\mathrm{I}+1)+\mathrm{FX} 2(\mathrm{I}+2)) * \mathrm{FX} 3(\mathrm{I}+2) \\
& \mathrm{D} 1=\mathrm{C} 1+(\mathrm{KSQ}(\mathrm{I})+\mathrm{KSQ}(\mathrm{I}+1)) / 12.0 \\
& \mathrm{D} 2=\mathrm{C} 2+(\mathrm{KSQ}(\mathrm{I})+6.0 * \mathrm{KSQ}(\mathrm{I}+1)+\mathrm{KSQ}(\mathrm{I}+2)) / 12.0 \\
& \mathrm{D} 3=\mathrm{C} 3+(\mathrm{KSQ}(\mathrm{I}+1)+\mathrm{KSQ}(\mathrm{I}+2)) / 12.0
\end{aligned}
$$

C

C

DO $3 \mathrm{~J}=1$, NPADE

$\mathrm{E} 1=\mathrm{K} 0 * * 2 * \mathrm{~A} 1+\mathrm{PD} 2(\mathrm{~J}) * \mathrm{D} 1$

$\mathrm{E} 2=\mathrm{K} 0 * * 2 * \mathrm{~A} 2+\mathrm{PD} 2(\mathrm{~J}) * \mathrm{D} 2$

$\mathrm{E} 3=\mathrm{K} 0 * * 2 * \mathrm{~A} 3+\mathrm{PD} 2(\mathrm{~J}) * \mathrm{D} 3$

$\mathrm{F} 1=\mathrm{PD} 1(\mathrm{~J}) * \mathrm{CI} * \mathrm{~K} 0 * \mathrm{D} 1$

$\mathrm{F} 2=\mathrm{PD} 1(\mathrm{~J}) * \mathrm{CI} * \mathrm{~K} 0 * \mathrm{D} 2$

$\mathrm{F} 3=\mathrm{PD} 1(\mathrm{~J}) * \mathrm{CI} * \mathrm{~K} 0 * \mathrm{D} 3$

$\mathrm{R} 1(\mathrm{I}, \mathrm{J})=\mathrm{E} 1-0.5 * \mathrm{DR} * \mathrm{~F} 1$

$\mathrm{R} 2(\mathrm{I}, \mathrm{J})=\mathrm{E} 2-0.5 * \mathrm{DR} * \mathrm{~F} 2$ 


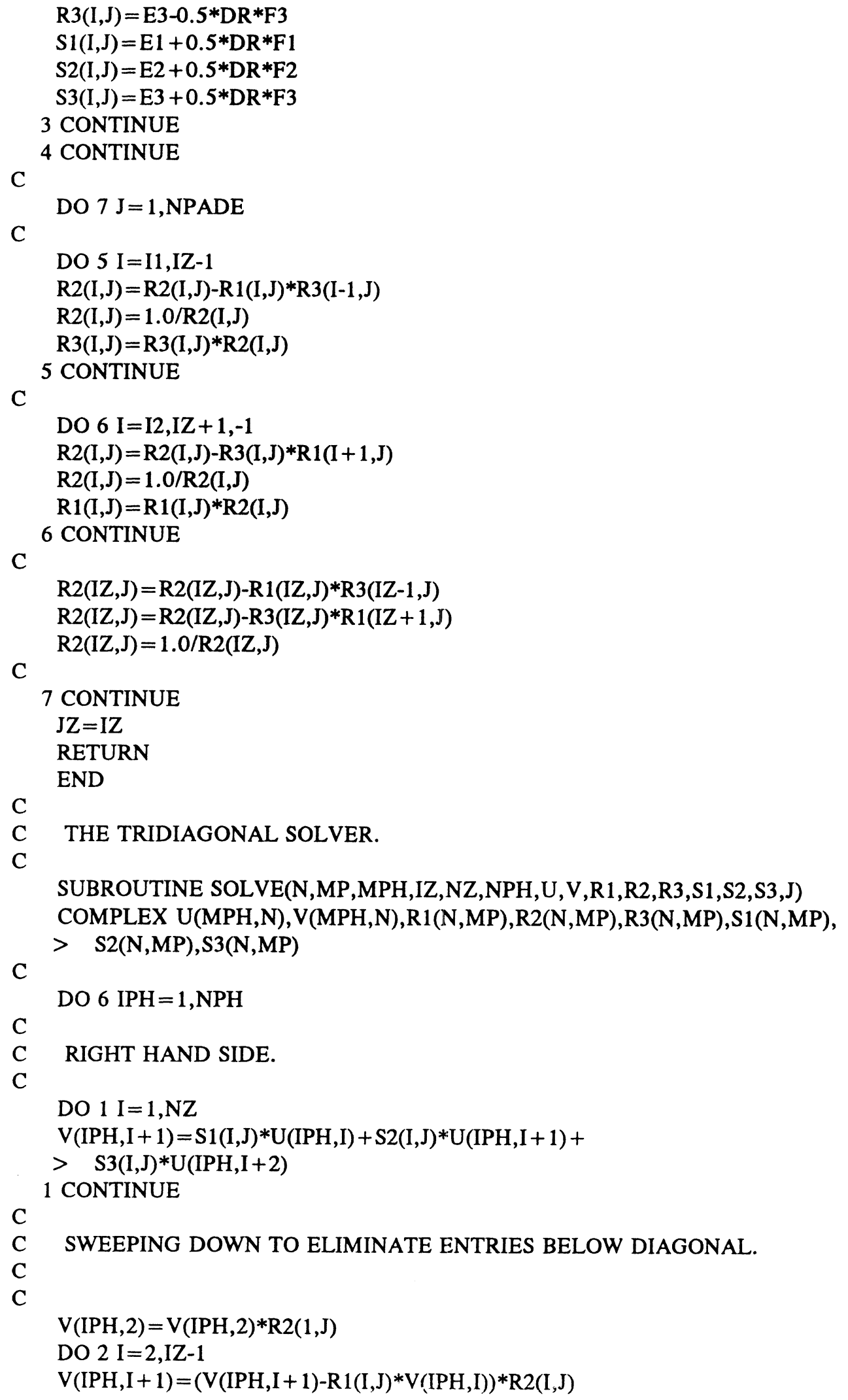




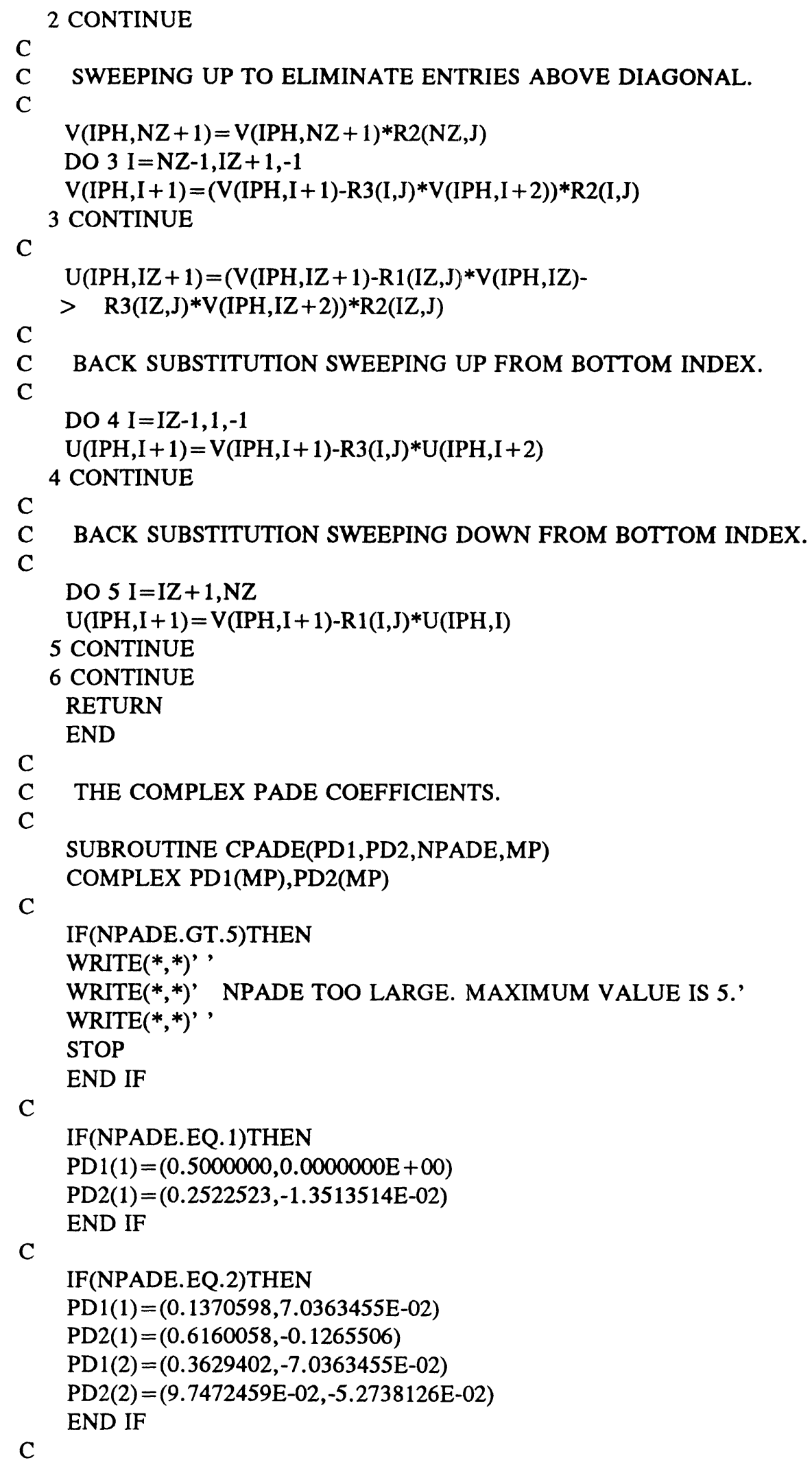


IF(NPADE.EQ.3)THEN

$\mathrm{PD} 1(1)=(5.4896813 \mathrm{E}-02,3.6742385 \mathrm{E}-03)$

$\mathrm{PD} 2(1)=(0.8086857,-9.1023222 \mathrm{E}-03)$

$\mathrm{PD} 1(2)=(0.1751380,3.1703257 \mathrm{E}-03)$

$\mathrm{PD} 2(2)=(0.3846456,-1.4611028 \mathrm{E}-02)$

$\operatorname{PD1}(3)=(0.2699652,-6.8445643 E-03)$

$\mathrm{PD} 2(3)=(4.8821609 \mathrm{E}-02,-2.9557836 \mathrm{E}-03)$

C

END IF

IF(NPADE.EQ.4)THEN

$\mathrm{PD} 1(1)=(2.5381334 \mathrm{E}-02,6.0041733 \mathrm{E}-03)$

$\mathrm{PD} 2(1)=(0.8832191,-1.8850777 \mathrm{E}-02)$

$\mathrm{PD} 1(2)=(8.7361716 \mathrm{E}-02,9.9593531 \mathrm{E}-03)$

$\mathrm{PD} 2(2)=(0.5945545,-4.3388147 \mathrm{E}-02)$

$\mathrm{PD} 1(3)=(0.1649370,-2.0355983 \mathrm{E}-03)$

$\mathrm{PD} 2(3)=(0.2610570,-3.0401150 \mathrm{E}-02)$

$\mathrm{PD} 1(4)=(0.2223199,-1.3927928 \mathrm{E}-02)$

$\mathrm{PD} 2(4)=(3.2206986 \mathrm{E}-02,-4.3234974 \mathrm{E}-03)$

C

END IF

\section{IF(NPADE.EQ.5)THEN}

$\mathrm{PD} 1(1)=(1.6475746 \mathrm{E}-02,2.6173585 \mathrm{E}-03)$

$\mathrm{PD} 2(1)=(0.9128319,-9.0011684 \mathrm{E}-03)$

$\mathrm{PD1}(2)=(5.7667062 \mathrm{E}-02,8.8840965 \mathrm{E}-03)$

$\mathrm{PD} 2(2)=(0.6861078,-3.0282630 \mathrm{E}-02)$

$\operatorname{PD} 1(3)=(0.1035779,1.0283243 \mathrm{E}-02)$

$\mathrm{PD} 2(3)=(0.4076422,-4.4838917 \mathrm{E}-02)$

$\mathrm{PD} 1(4)=(0.1452988,-4.1513294 \mathrm{E}-03)$

$\mathrm{PD} 2(4)=(0.1658151,-2.9934738 \mathrm{E}-02)$

$\mathrm{PD} 1(5)=(0.1769805,-1.7633369 \mathrm{E}-02)$

$\mathrm{PD} 2(5)=(1.9913642 \mathrm{E}-02,-4.2300178 \mathrm{E}-03)$

END IF

C

RETURN

END

C

C THE ENERGY IS EVALUATED.

C

SUBROUTINE ENERGY(UREP,UDAT,NPH,E)

COMPLEX UREP(100),UDAT(100),RD

C

$\mathrm{AMP}=0.0$

$\mathrm{RD}=\mathrm{CMPLX}(0.0,0.0)$

DO $1 \mathrm{IPH}=1, \mathrm{NPH}$

$\mathrm{AMP}=\mathrm{AMP}+\mathrm{CABS}(\mathrm{UREP}(\mathrm{IPH}))^{* * 2}$

$\mathrm{RD}=\mathrm{RD}+\mathrm{UREP}(\mathrm{IPH}) * \mathrm{UDAT}$ (IPH)

1 CONTINUE

$\mathrm{E}=\mathrm{CABS}(\mathrm{RD})^{* * 2} / \mathrm{AMP}$

C

RETURN

END 
$\mathrm{C}$

C THE ENVIRONMENT IS PARMETRIZED.

C SUBROUTINE PARMTR(F,G,NF,CW,CB, RHOB, ATTN,N)

C REAL CW(N), CB(N), RHOB(N),ATTN(N),F(100), G(100)

$$
\begin{aligned}
& \mathrm{DZ}=\mathrm{G}(1) \\
& \mathrm{ZMAX}=\mathrm{G}(2) \\
& \mathrm{NZ}=\operatorname{IFIX}(\mathrm{G}(3))
\end{aligned}
$$

C

$$
\mathrm{Z}=0.0
$$

DO $1 \mathrm{I}=1, \mathrm{NZ}+2$

$\mathrm{CW}(\mathrm{I})=\mathrm{F}(3)+(\mathrm{F}(4)-\mathrm{F}(3)) * \mathrm{Z} / \mathrm{F}(1)$

$\mathrm{CB}(\mathrm{I})=\mathrm{F}(5)+(\mathrm{Z}-\mathrm{F}(1)) *(\mathrm{~F}(6)-\mathrm{F}(5)) /(\mathrm{ZMAX}-\mathrm{F}(1))$

$\mathrm{RHOB}(\mathrm{I})=\mathrm{F}(7)$

$\operatorname{ATTN}(\mathrm{I})=\mathrm{F}(8)$

$\mathrm{Z}=\mathrm{Z}+\mathrm{DZ}$

1 CONTINUE

C

RETURN

END

$\mathrm{C}$

C THIS SUBROUTINE INVERTS MATRIX A.

$\mathrm{C}$

SUBROUTINE INVERT(KMAT,N)

COMPLEX*16 A(100,100), B(100,100)

C

COMPLEX KMAT $(100,100)$

DO $2 \mathrm{I}=1, \mathrm{~N}$

DO $1 \mathrm{~J}=1, \mathrm{~N}$

$\mathrm{A}(\mathrm{I}, \mathrm{J})=\mathrm{KMAT}(\mathrm{I}, \mathrm{J})$

$\mathrm{B}(\mathrm{I}, \mathrm{J})=0.0$

1 CONTINUE

$\mathrm{B}(\mathrm{I}, \mathrm{I})=1.0$

2 CONTINUE

C

$\mathrm{I}=1$

3 CALL PIVOT(A,B,I,N)

CALL MULT(A,B,I,N)

CALL ADD1 $(A, B, I, N)$

$\mathrm{I}=\mathrm{I}+1$

IF(I.EQ.N)GO TO 4

GO TO 3

4 CALL MULT(A,B,I,N)

5 CALL ADD2(A,B,I,N)

IF(I.EQ.2)GO TO 6

$\mathrm{I}=\mathrm{I}-1$

C

GO TO 5

$$
6 \text { DO } 8 \mathrm{I}=1, \mathrm{~N}
$$

DO $7 \mathrm{~J}=1, \mathrm{~N}$ 


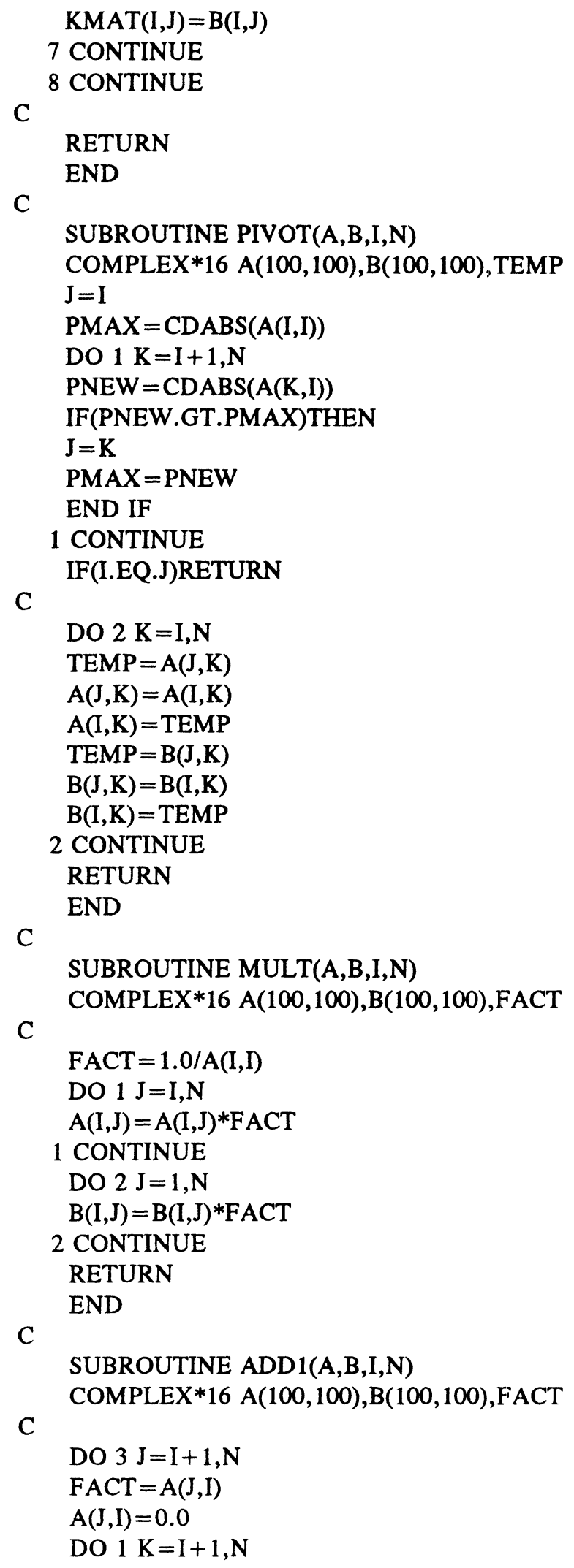




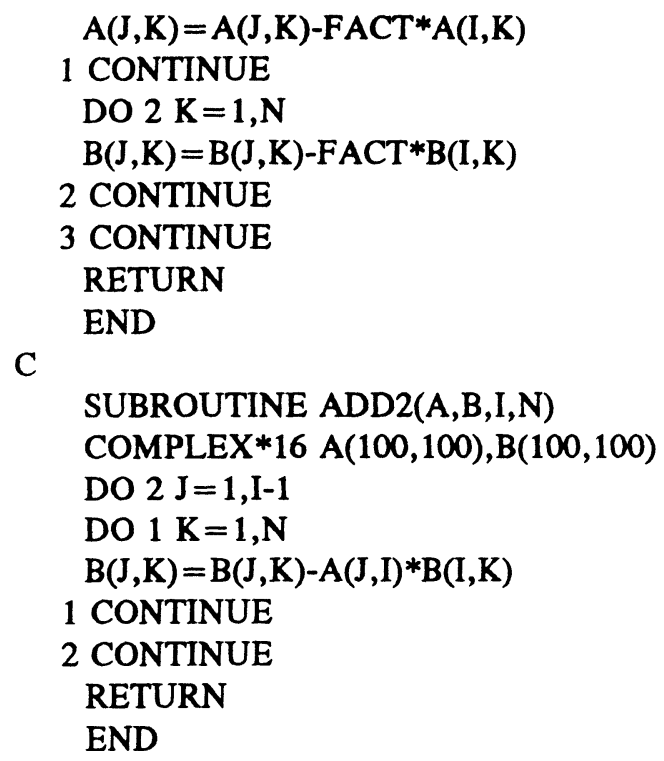




\section{APPENDIX C DOA Estimation Algorithm Code}

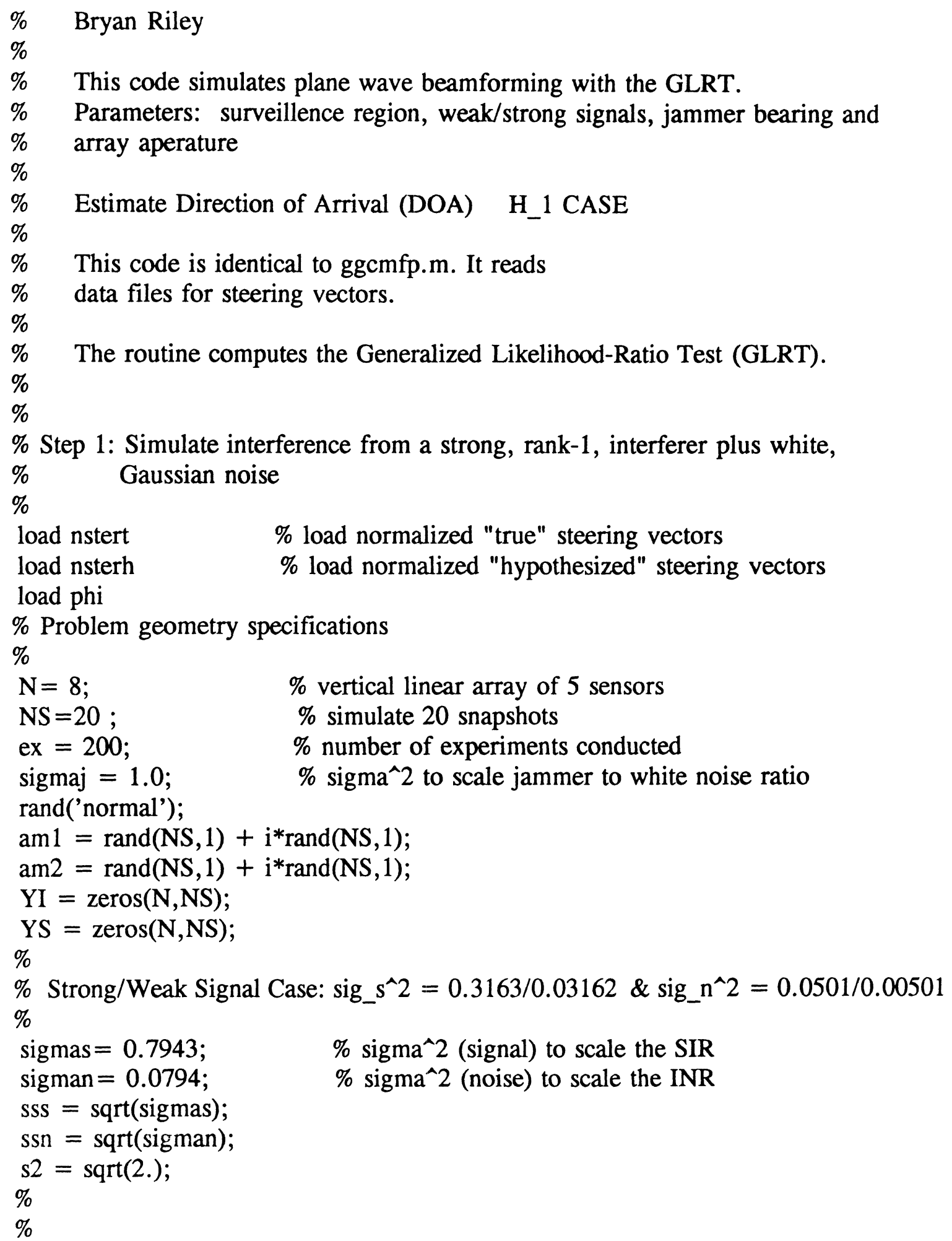


$\%$

\% Step 1: Simulate a point source of interest plus interference plus white

$\%$

$\% \quad$ Gaussian noise and form estimated array covariance matrix

$\%$

$\%$

H_1 Case: NO SIGNAL PRESENT, YS_data matrix

ex $=200$

doa $=11$;

for io $=1:$ ex

for $\mathrm{sp}=1: \mathrm{doa}$

\% surveillence region

$\mathrm{YS}=\operatorname{zeros}(\mathrm{N}, \mathrm{NS})$;

for $\mathrm{k}=1: \mathrm{NS}$

$\mathrm{YS}(:, \mathrm{k})=(\operatorname{am} 1(\mathrm{k}, 1) * \operatorname{nstert}(:, 1)) / \mathrm{s} 2+\left(\operatorname{ssn} * \operatorname{rand}(\mathrm{N}, 1)+\mathrm{i}{ }^{*} \operatorname{ssn} * \operatorname{rand}(\mathrm{N}, 1)\right) / \mathrm{s} 2+\ldots$

end $((\mathrm{sss} * a m 2(\mathrm{k}, 1)) *$ nstert $(:, 2)) / \mathrm{s} 2$;

$\%$

tstYS $=(1 . / N S)^{*}\left(Y^{*}\right.$ YS $\left.^{\prime}\right)$

$\% \quad$ RANK1 $=$ svd(tstYS), pause

\% Step 2: Simulate under H_o R, the ML estimate -

$\% \quad$ Start of GLRT for Gaussian assumption

$\%$

mkY $=1 ; \quad \%$ rank 1 interference data matrix

$\operatorname{sum} 1=\operatorname{zeros}(\mathbf{N}, \mathbf{N})$;

$\operatorname{sum} 2=\operatorname{zeros}(\mathbf{N}, \mathbf{N})$

sigho $=0.0$

So $=\left(\mathrm{YS}^{*} \mathrm{YS}^{\prime}\right)^{*}(1 . / \mathrm{NS}) ; \quad \%$ change YS to YI for signal not present case

$[\mathrm{ROV}, \mathrm{ROS}, \mathrm{ROU}]=\operatorname{svd}(\mathrm{So})$;

ROS1 = ROS;

$\mathrm{k}=\mathrm{rnkY}+1$;

for $\mathrm{i}=\mathrm{k}: \mathrm{N}$

$\operatorname{ROS} 1(\mathrm{i}, \mathrm{i})=0.0$;

end

sum1 $=\mathrm{ROV} * \mathrm{ROS} 1 *$ ROU';

$\mathrm{k}=\mathrm{rnkY}+1$;

for $\mathrm{i}=\mathrm{k}: \mathrm{N}$

sigho = ROS $(\mathrm{i}, \mathrm{i})+$ sigho;

$\left.\operatorname{sum} 2=\operatorname{sum} 2+(\operatorname{ROV}(:, \mathrm{i}) *(\operatorname{ROU}(:, \mathrm{i})))^{\prime}\right)$;

end

ssho $=$ sigho/(N -rnkY);

REST0 = sum 1 + (ssho * sum2); \% Maximum likelihood estimate of R under H_o hypo = svd(REST0);

$\%$

\% Step 2a: Simulate under H_1 R, the ML estimate - 
\% Start of GLRT for Gaussian assumption

\% Step 2b: Compute the Fourier Transform of column 10 of the reduced rank data matrix $\mathrm{Y}$

$\%$

plus signal sequence

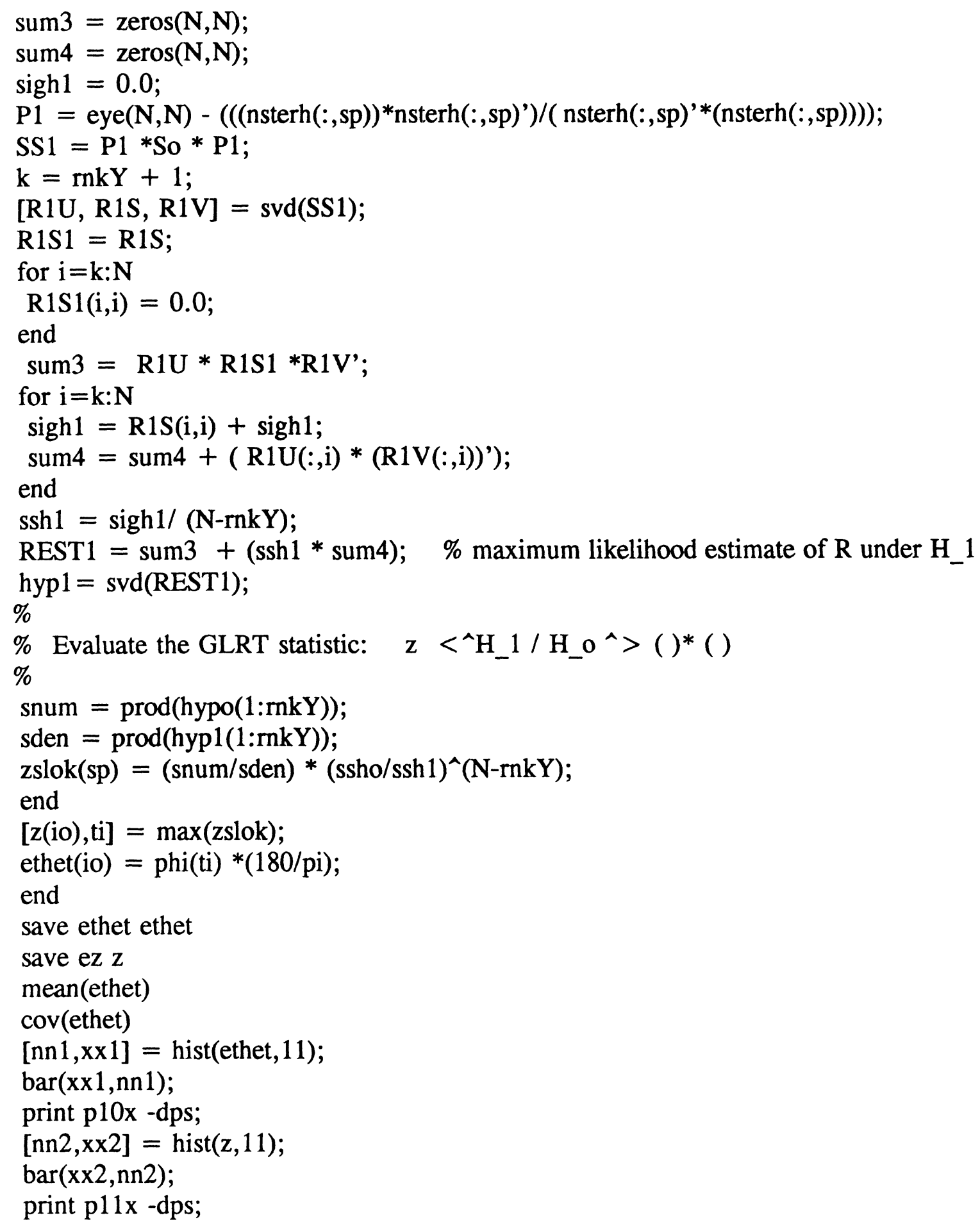


plot((phi*180./pi),zslok);

$\%$ title ('N $=16, v s^{\wedge} 2=.7943, v n^{\wedge} 2=0.07943, \mathrm{vj}^{\wedge}=0$ ’);

xlabel('DOA in Degrees');

ylabel('z Statistic');

print doalok -dps

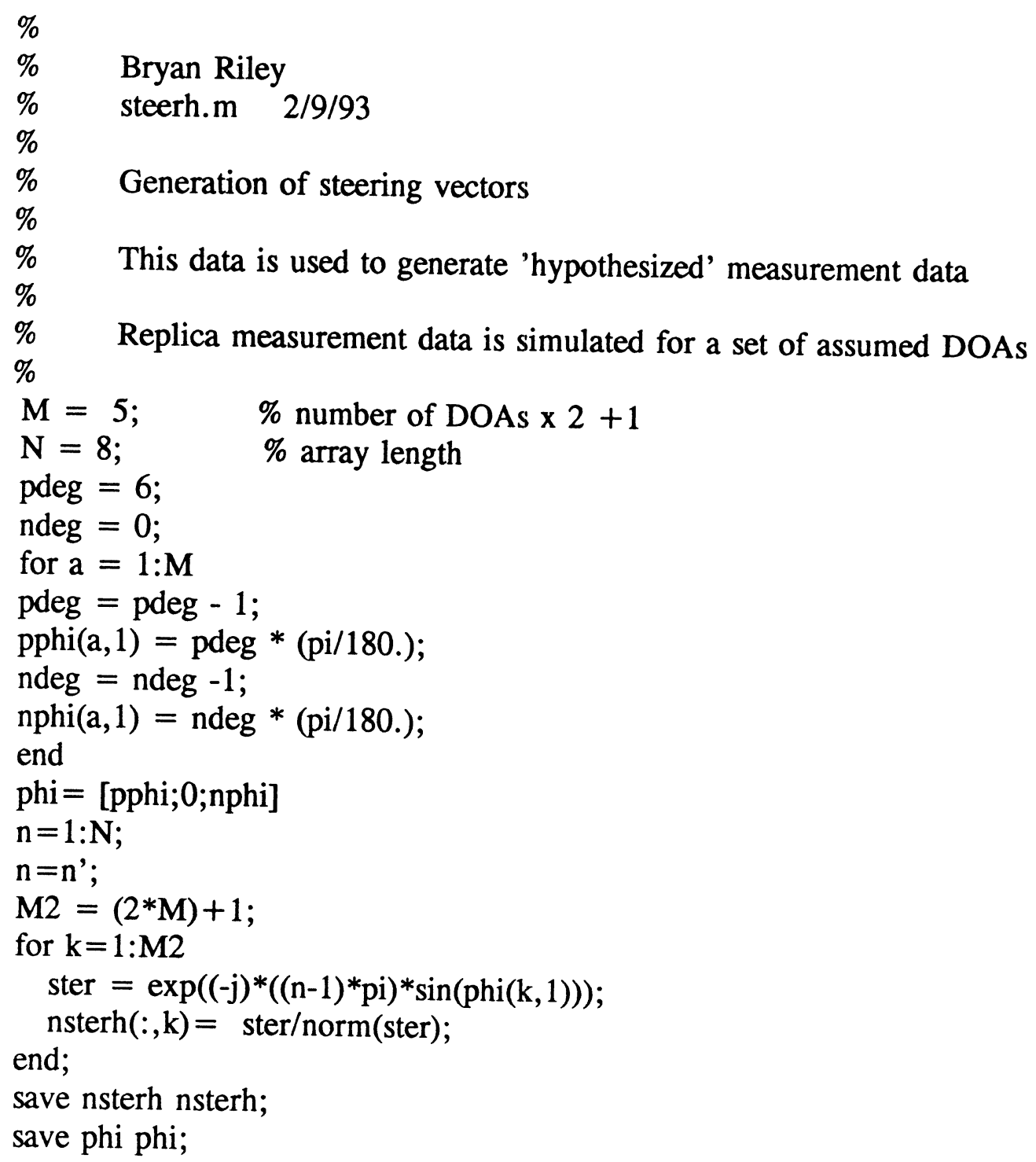




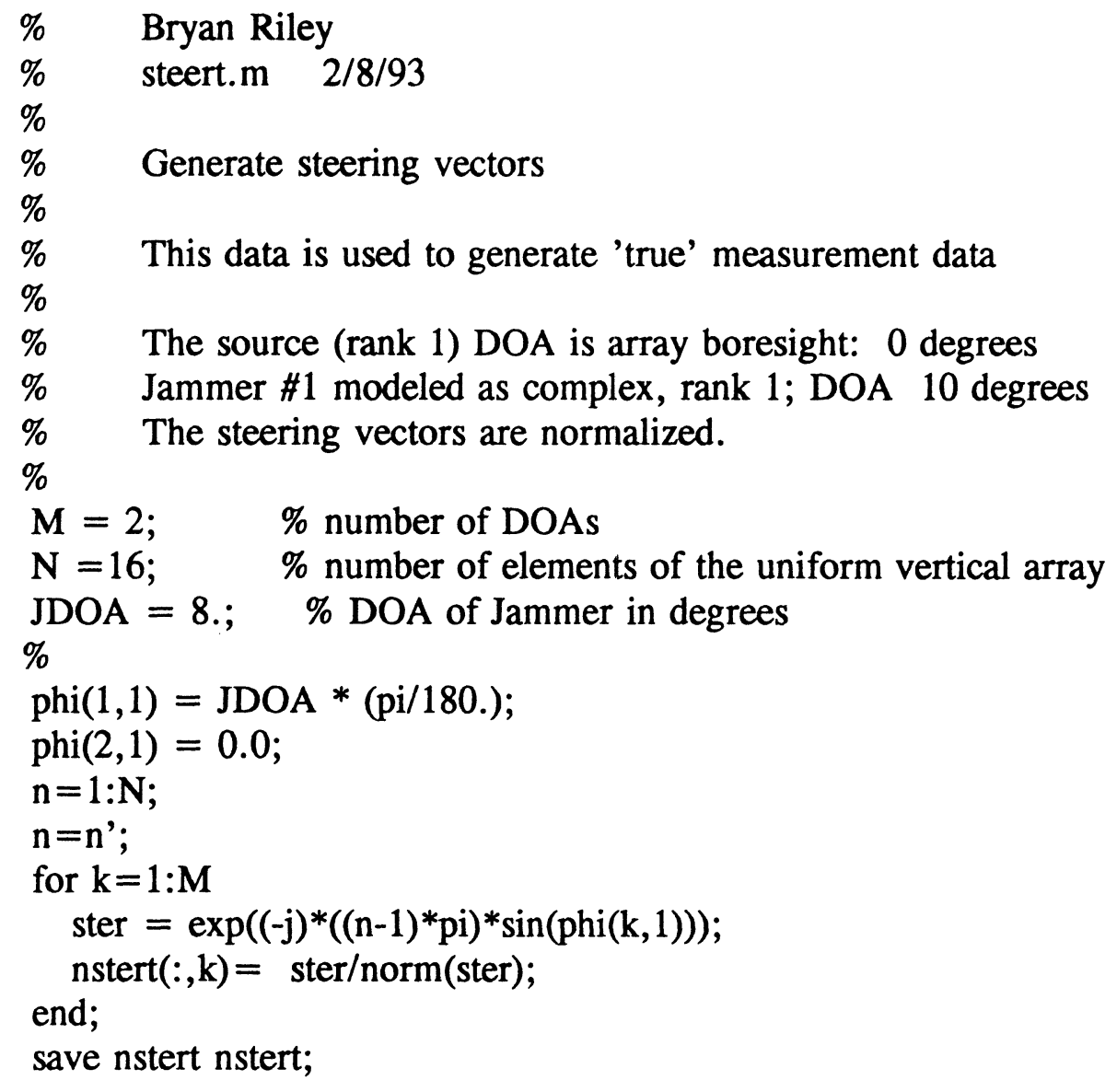




\section{APPENDIX D ROC Computational Code}

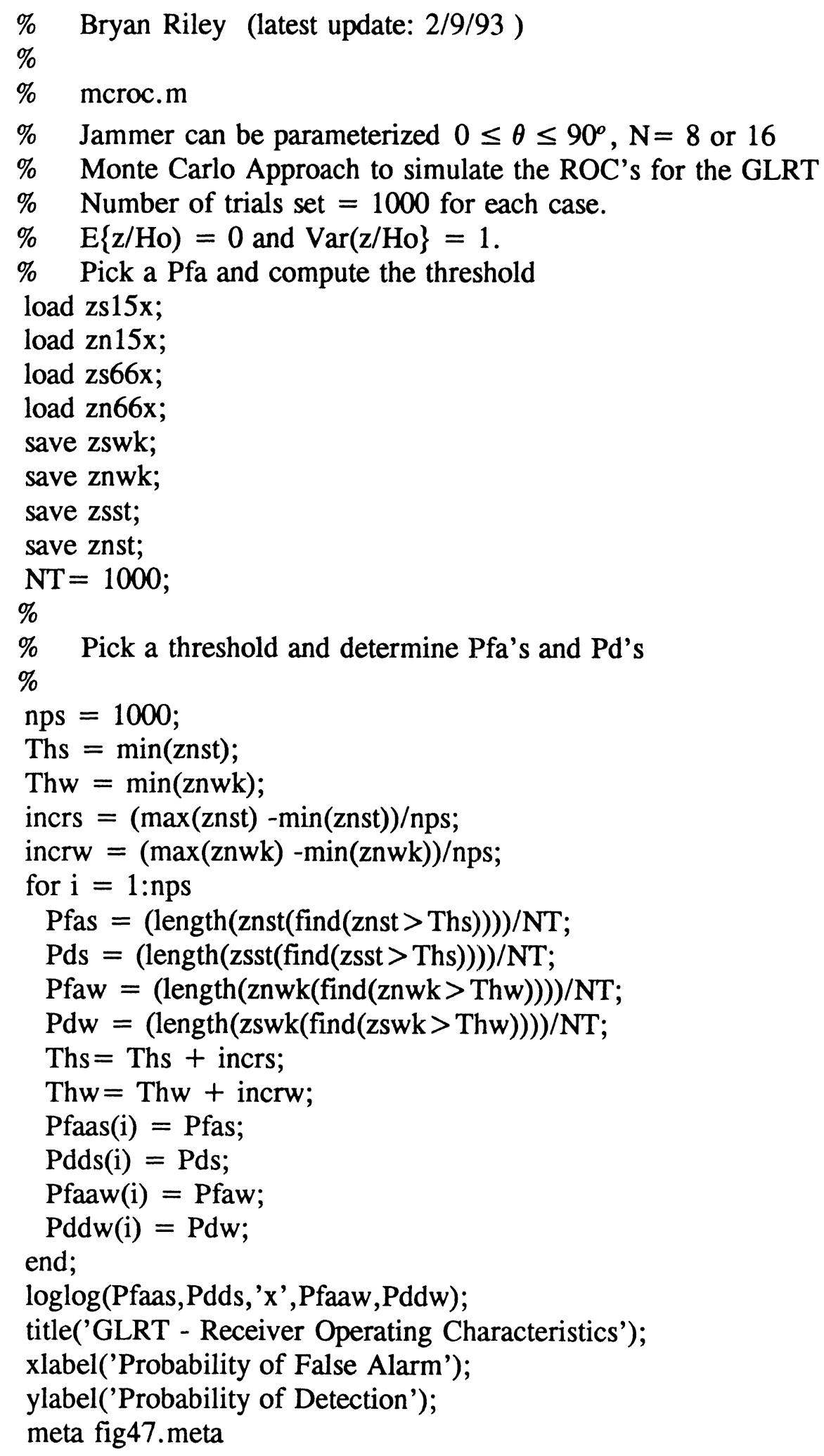




\section{APPENDIX E Modified GLRT Code To Investigate Subspace Decomposition}

$\%$

$\%$

$\%$

$\%$

$\%$

$\%$

$\%$

$\%$

$\%$

$\%$

$\%$

$\%$

$\%$

\% Step 1: Simulate interference from a strong, rank-1, interferer plus white, $\%$

$\%$

load nstert

load nsterh6

load phi6

$\%$

$\%$

$\mathrm{N}=16$

$\mathrm{NS}=20$

ex $=1000$;

sigmaj $=1.0$

rand('normal');

$\mathrm{am} 1=\operatorname{rand}(\mathrm{NS}, 1)+\mathrm{i} * \operatorname{rand}(\mathrm{NS}, 1)$;

$\mathrm{am} 2=\operatorname{rand}(\mathrm{NS}, 1)+\mathrm{i}^{*} \operatorname{rand}(\mathrm{NS}, 1)$

$\mathrm{YI}=\operatorname{zeros}(\mathrm{N}, \mathrm{NS})$

$\mathrm{YS}=\operatorname{zeros}(\mathrm{N}, \mathrm{NS})$;

$\%$

$\%$ Strong/Weak Signal Case: sig_s^2 $=0.3163 / 0.03162 \&$ sig_n^2 $=0.0501 / 0.00501$ $\%$

sigmas $=.1766$

sigman $=0.2799$

$\%$ sigma^2 (signal) to scale the SIR

sss $=$ sqrt(sigmas);

ssn $=\operatorname{sqrt}($ sigman $)$;

$\mathrm{s} 2=\operatorname{sqrt}(2$.

$\%$

$\%$

$\%$
$\%$ load normalized "true" steering vectors

\% load normalized "hypothesized" steering vectors
$\%$ vertical linear array of 5 sensors

$\%$ simulate 20 snapshots

$\%$ number of experiments conducted

$\%$ sigma^2 to scale jammer to white noise ratio 


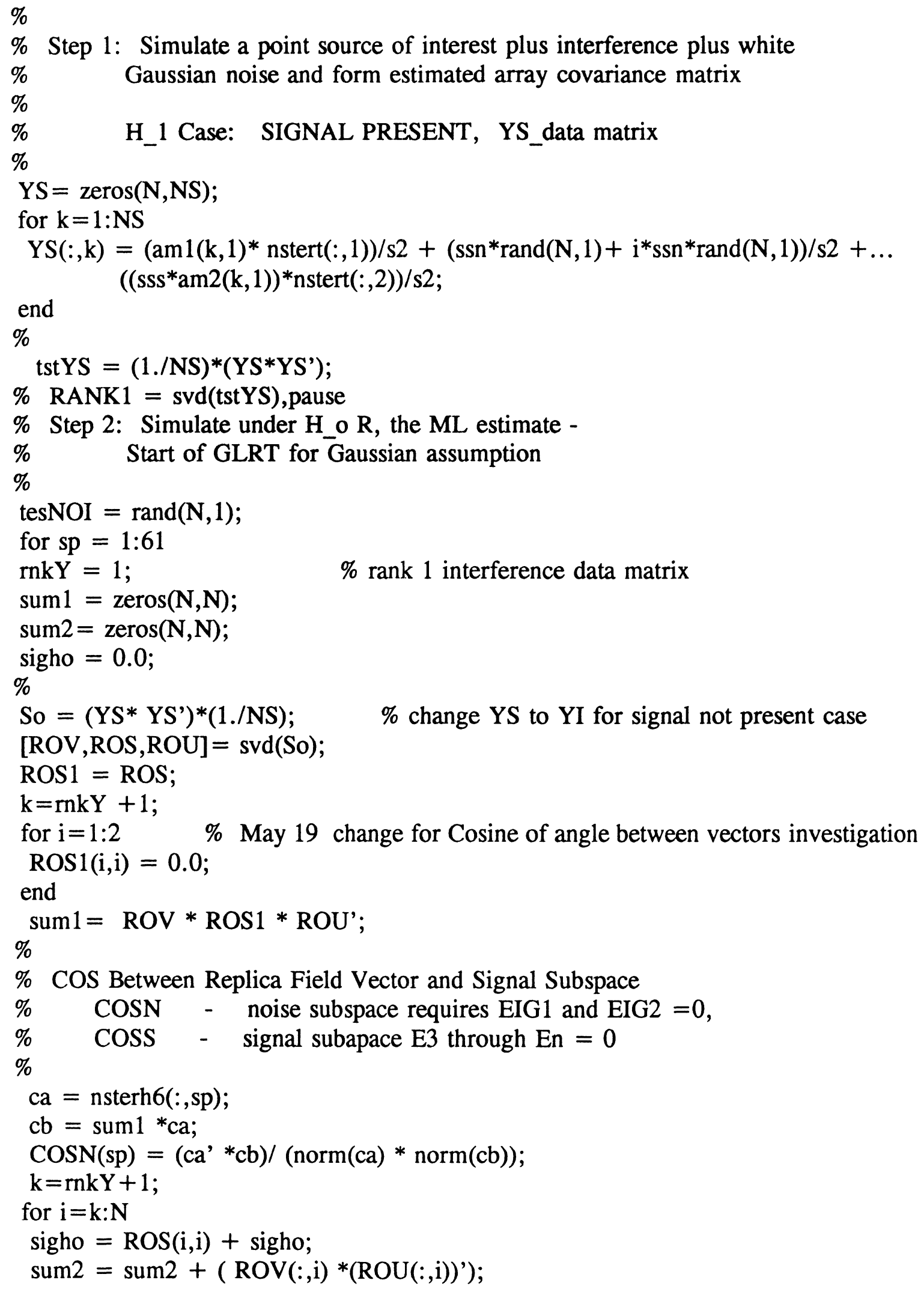




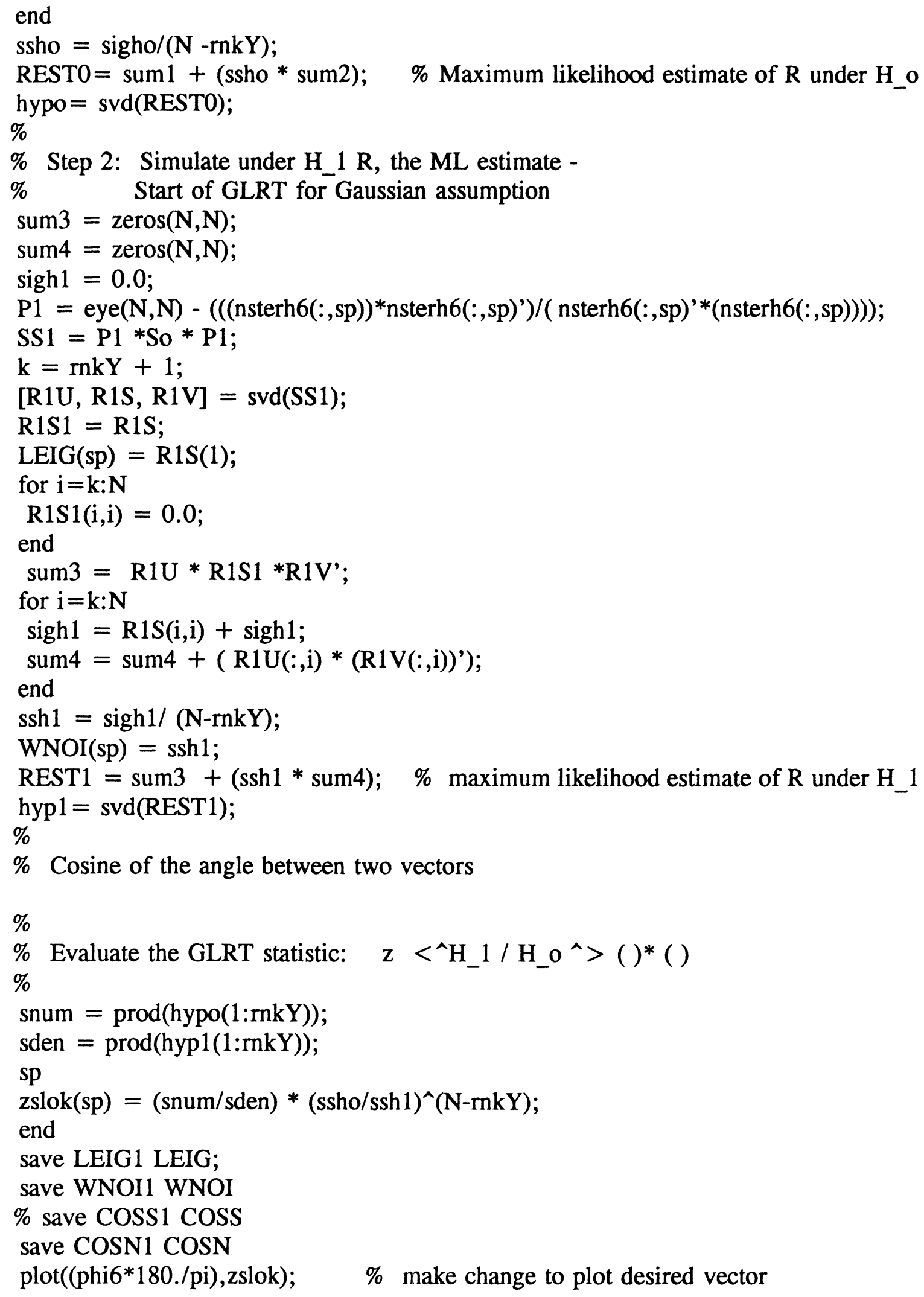


$\%$ ylabel('Largest Eigenvalue of $\mathrm{P} * \mathrm{~S} 1 * \mathrm{P} ')$;

$\%$ title('H1 Assumption: White Noise Estimate');

ylabel(' GLRT OUTPUT ')

$\%$ title('Cosine Between Replica Field Vector and Signal Subspace')

xlabel('Assumed DOA in Degrees');

meta glrto.met 
APPENDIX F P/E Solver, Bartlett, and MLM Computational Code

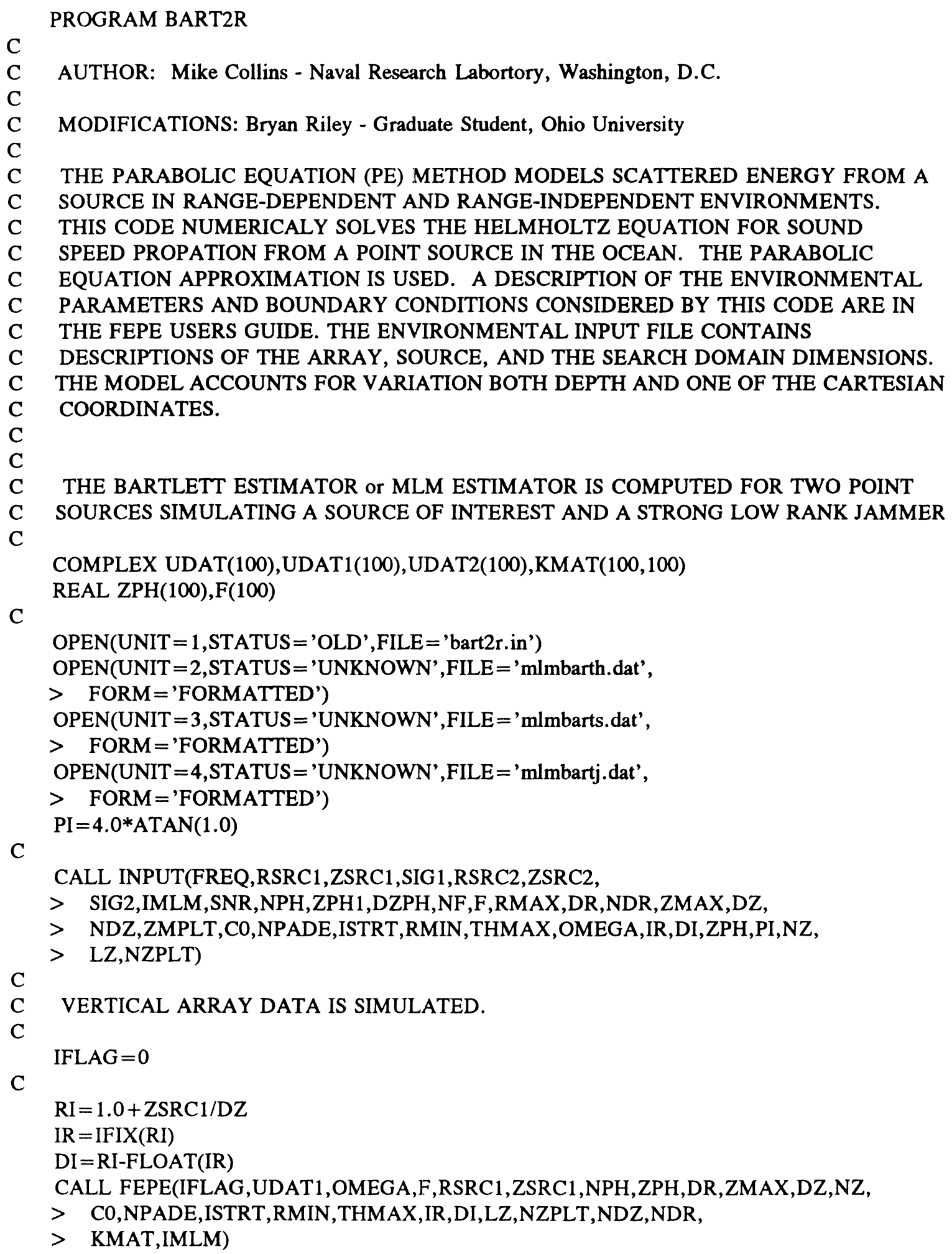


C

$\mathrm{RI}=1.0+Z S R C 2 / \mathrm{DZ}$

$\mathrm{IR}=\mathrm{IFIX}(\mathrm{RI})$

$\mathrm{DI}=\mathrm{RI}-\mathrm{FLOAT}(\mathrm{IR})$

CALL FEPE(IFLAG,UDAT2,OMEGA,F,RSRC2,ZSRC2,NPH,ZPH,DR,ZMAX,DZ,NZ, $>$ C0,NPADE,ISTRT,RMIN,THMAX,IR,DI,LZ,NZPLT,NDZ,NDR,

$\mathrm{C}$

$>$ KMAT,IMLM)

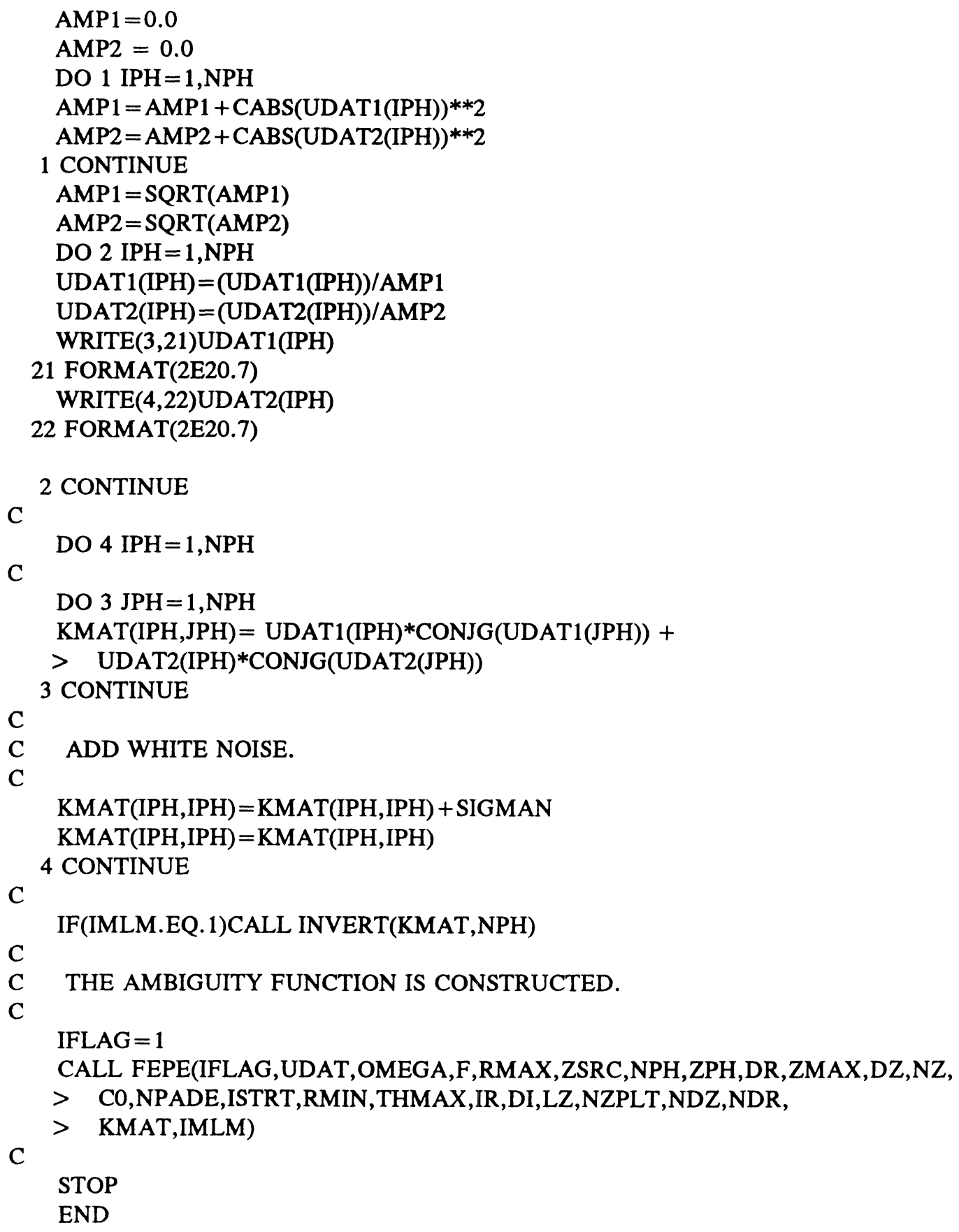




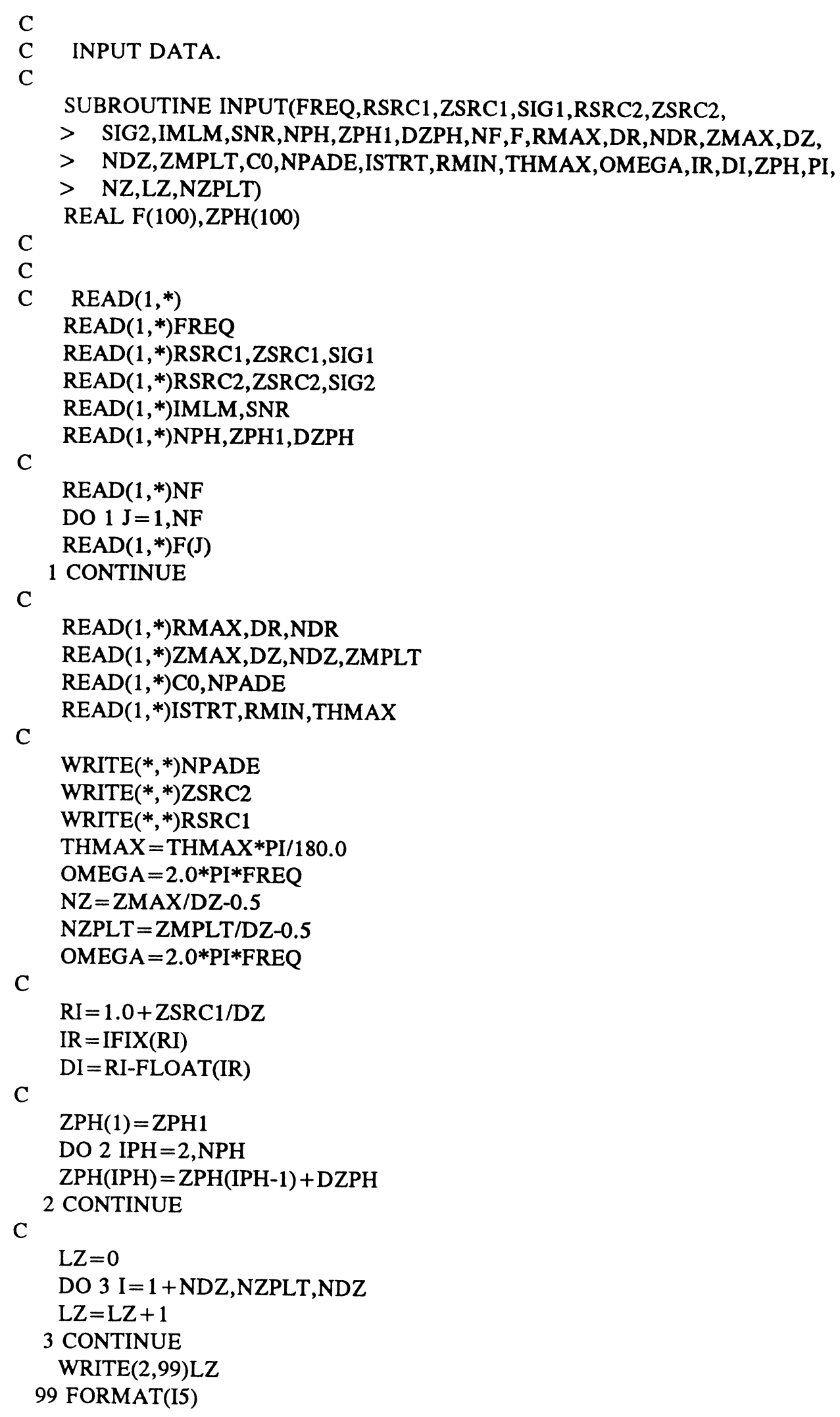


C

RETURN

END

$\mathrm{C}$

C THE PE SOLVER.

C

SUBROUTINE FEPE(IFLAG,UDAT,OMEGA,F,RMAX,ZSRC,NPH,ZPH,DR,ZMAX,DZ,

$>$ NZ,C0,NPADE,ISTRT,RMIN,THMAX,IR,DI,LZ,NZPLT,NDZ,NDR,

$\mathrm{C}$

$>$ KMAT,IMLM)

COMPLEX CI,KSQB,KSQ,U,V,UR1,UR2,R1,R2,R3,S1,S2,S3,PD1,PD2,

$>\operatorname{UDAT}(100), \mathrm{UREP}(100), \operatorname{KMAT}(100,100)$, ETEMP,UTEMP

REAL K0,KSQW,KZS,F(100),G(100),ZPH(100),EN(5000)

PARAMETER $(\mathrm{N}=2000, \mathrm{MP}=3, \mathrm{MPH}=16)$

DIMENSION CW(N), CB(N), RHOB(N), ATTN(N),KSQW(N),KSQB(N),KSQ(N),

$>$ FX1(N),FX2(N),FX3(N),U(MPH,N),V(MPH,N),PD1(MP),PD2(MP),

C

$>\quad \mathrm{R} 1(\mathrm{~N}, \mathrm{MP}), \mathrm{R} 2(\mathrm{~N}, \mathrm{MP}), \mathrm{R} 3(\mathrm{~N}, \mathrm{MP}), \mathrm{S} 1(\mathrm{~N}, \mathrm{MP}), \mathrm{S} 2(\mathrm{~N}, \mathrm{MP}), \mathrm{S} 3(\mathrm{~N}, \mathrm{MP})$

INTEGER count

count $=0$.

$\mathrm{PI}=4.0 * \operatorname{ATAN}(1.0)$

$\mathrm{CI}=\operatorname{CMPLX}(0.0,1.0)$

$\mathrm{R}=0.0$

$\mathrm{ETA}=1.0 /(40.0 * \mathrm{PI} * \mathrm{ALOG} 10(\mathrm{EXP}(1.0)))$

$\mathrm{MDR}=0$

$\mathrm{IZSRC}=1.5+\mathrm{ZSRC} / \mathrm{DZ}$

C

CALL CPADE(PD1,PD2,NPADE,MP)

$\mathrm{K} 0=\mathrm{OMEGA} / \mathrm{C} 0$

DO $1 \mathrm{IPH}=1, \mathrm{NPH}$

$\mathrm{V}(\mathrm{IPH}, 1)=0.0$

$\mathrm{V}(\mathrm{IPH}, \mathrm{NZ}+2)=0.0$

C

1 CONTINUE

C SET UP PARAMETRIZATION.

C

$\mathrm{Z}=\mathrm{F}(1)+(\mathrm{F}(2)-\mathrm{F}(1)) * \mathrm{R} / \mathrm{RMAX}$

$\mathrm{G}(1)=\mathrm{DZ}$

$\mathrm{G}(2)=\mathrm{ZMAX}$

$\mathrm{G}(3)=\mathrm{FLOAT}(\mathrm{NZ})+0.5$

CALL PARMTR(F,G,NF,CW,CB, RHOB, ATTN,N)

DO $2 \mathrm{I}=1, \mathrm{NZ}+2$

$\mathrm{KSQW}(\mathrm{I})=(\mathrm{OMEGA} / \mathrm{CW}(\mathrm{I})) * * 2$

$\mathrm{KSQB}(\mathrm{I})=((\mathrm{OMEGA} / \mathrm{CB}(\mathrm{I})) *(1.0+\mathrm{CI} * \mathrm{ETA} * \mathrm{ATTN}(\mathrm{I}))) * * 2$

2 CONTINUE

C

C SET UP PE STARTING FIELDS.

C

DO $3 \mathrm{IPH}=1, \mathrm{NPH}$

$\mathrm{IZS}=1.5+\mathrm{ZPH}(\mathrm{IPH}) / \mathrm{DZ}$

$\mathrm{KZS}=\mathrm{OMEGA} / \mathrm{CW}(\mathrm{IZS})$ 


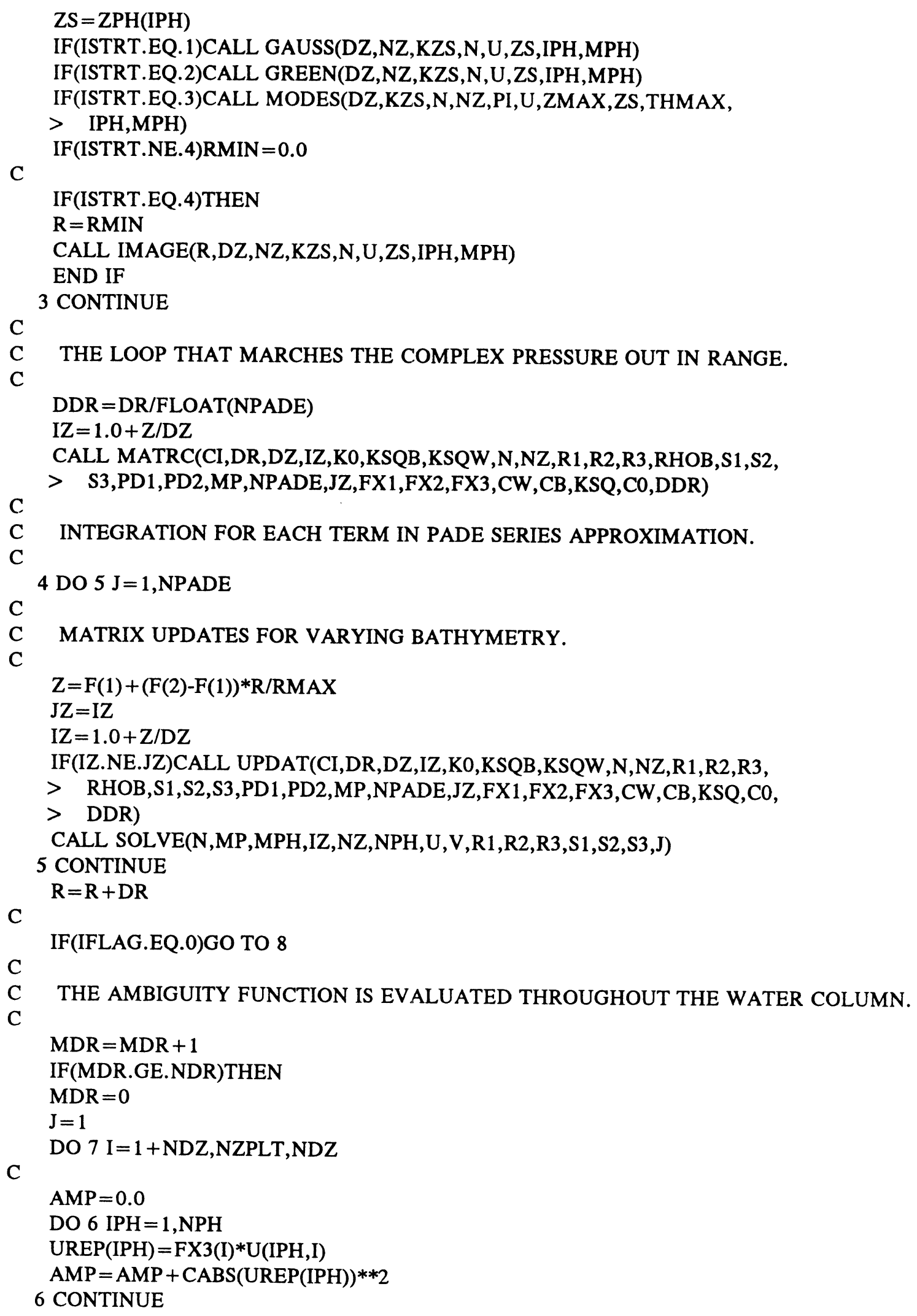




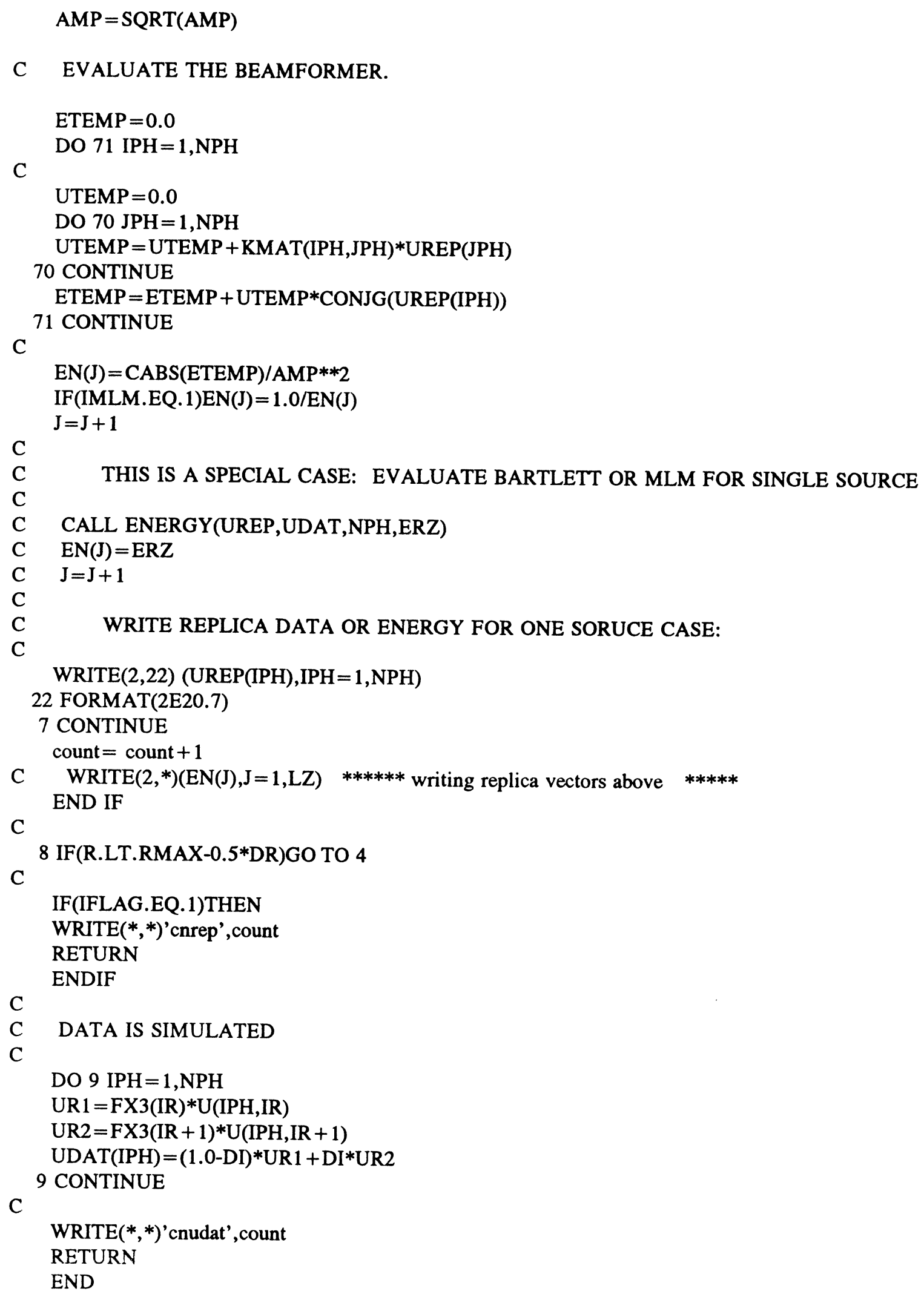




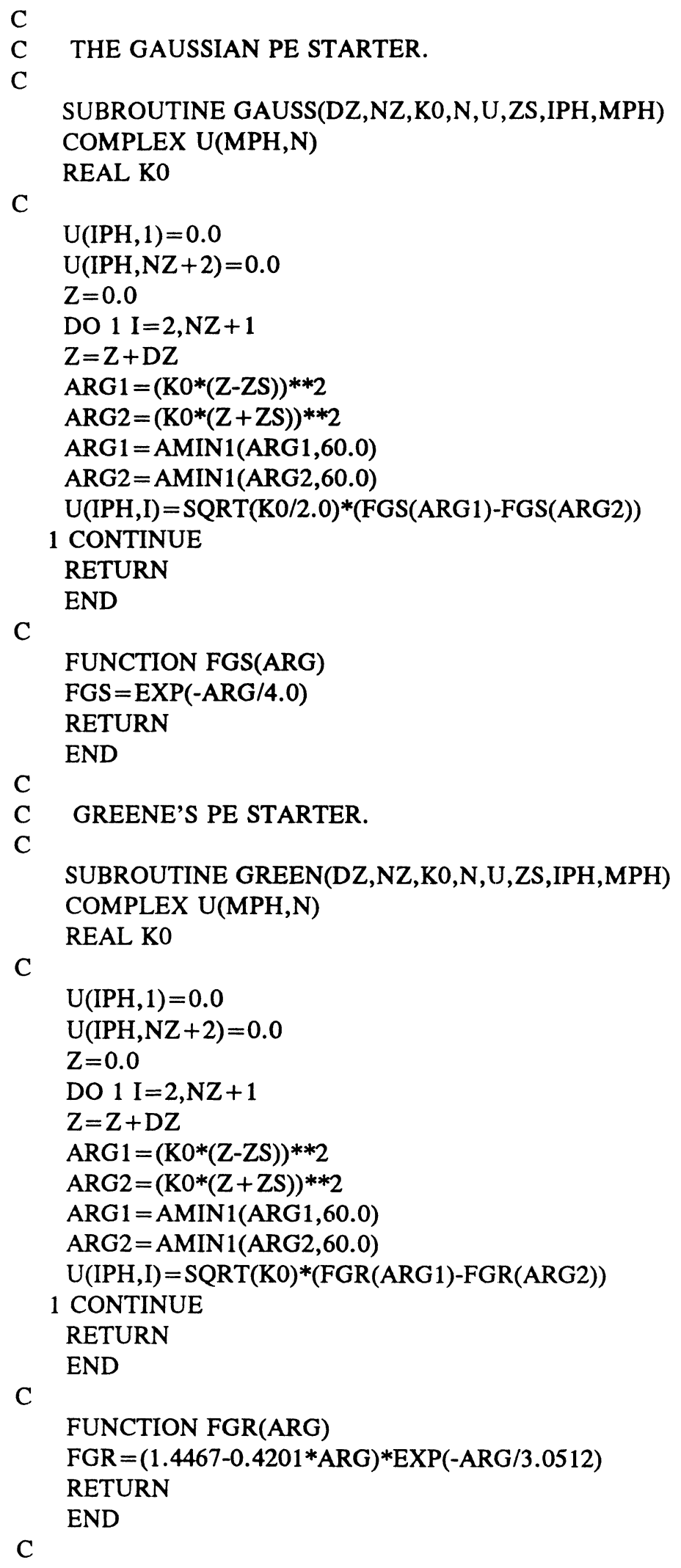


C THE MODAL PE STARTER.

C SUBROUTINE MODES(DZ,K0,N,NZ,PI,U,ZMAX,ZS,THMAX,IPH,MPH) COMPLEX U(MPH,N)

$\mathrm{C}$ REAL KO,KR,KZ

DO $1 \mathrm{I}=1, \mathrm{NZ}+2$

$\mathrm{U}(\mathrm{IPH}, \mathrm{I})=0.0$

1 CONTINUE

$\mathrm{KZ}=\mathrm{PI} / \mathrm{ZMAX}$

$\mathrm{DKZ}=\mathrm{PI} / \mathrm{ZMAX}$

2 IF(KZ.GE.K0)RETURN

THETA $=$ ASIN $(K Z / K 0)$

IF(THETA.GT.THMAX)RETURN

$\mathrm{KR}=\mathrm{SQRT}(\mathrm{K} 0 * * 2-\mathrm{KZ} * * 2)$

$\mathrm{Z}=\mathrm{DZ}$

$\mathrm{A}=\mathrm{SQRT}(2.0 * \mathrm{PI} / \mathrm{KR}) * \mathrm{SIN}(\mathrm{KZ} * \mathrm{ZS}) * 2.0 / \mathrm{ZMAX}$

DO $3 \mathrm{I}=2, \mathrm{NZ}+1$

$\mathrm{U}(\mathrm{IPH}, \mathrm{I})=\mathrm{U}(\mathrm{IPH}, \mathrm{I})+\mathrm{A} * \mathrm{SIN}(\mathrm{KZ} * \mathrm{Z})$

$\mathrm{Z}=\mathrm{Z}+\mathrm{DZ}$

3 CONTINUE

$\mathrm{KZ}=\mathrm{KZ}+\mathrm{DKZ}$

GO TO 2

END

C

C THE IMAGE FIELD PE STARTER.

C

SUBROUTINE IMAGE(R,DZ,NZ,K0,N,U,ZS,IPH,MPH)

COMPLEX U(MPH,N),CI

REAL K0

$\mathrm{CI}=\operatorname{CMPLX}(0.0,1.0)$

C

$\mathrm{U}(\mathrm{IPH}, 1)=0.0$

$\mathrm{U}(\mathrm{IPH}, \mathrm{NZ}+2)=0.0$

$\mathrm{Z}=0.0$

DO $1 \mathrm{I}=2, \mathrm{NZ}+1$

$\mathrm{Z}=\mathrm{Z}+\mathrm{DZ}$

$\mathrm{R} 1=\mathrm{SQRT}(\mathrm{R} * * 2+(\mathrm{Z}-\mathrm{ZS}) * * 2)$

$\mathrm{R} 2=\mathrm{SQRT}(\mathrm{R} * * 2+(\mathrm{Z}+\mathrm{ZS}) * * 2)$

$\mathrm{U}(\mathrm{IPH}, \mathrm{I})=\mathrm{SQRT}(\mathrm{R}) *(\mathrm{CEXP}(\mathrm{CI} * \mathrm{~K} 0 * \mathrm{R} 1) / \mathrm{R} 1-\mathrm{CEXP}(\mathrm{CI} * \mathrm{~K} 0 * \mathrm{R} 2) / \mathrm{R} 2)$

1 CONTINUE

RETURN

END

C

C MATRICES ARE SET UP WITH A SPECIAL DECOMPOSITION METHOD.

C

SUBROUTINE MATRC(CI,DR,DZ,IZ,K0,KSQB,KSQW,N,NZ,R1,R2,

$>$ R3,RHOB,S1,S2,S3,PD1,PD2,MP,NPADE,JZ,FX1,FX2,

$>\mathrm{FX} 3, \mathrm{CW}, \mathrm{CB}, \mathrm{KSQ}, \mathrm{C} 0, \mathrm{DDR})$

COMPLEX CI,D1,D2,D3,E1,E2,E3,F1,F2,F3,KSQB(N),KSQ(N), 
$>\mathrm{R} 1(\mathrm{~N}, \mathrm{MP}), \mathrm{R} 2(\mathrm{~N}, \mathrm{MP}), \mathrm{R} 3(\mathrm{~N}, \mathrm{MP}), \mathrm{S} 1(\mathrm{~N}, \mathrm{MP}), \mathrm{S} 2(\mathrm{~N}, \mathrm{MP}), \mathrm{S} 3(\mathrm{~N}, \mathrm{MP})$,

$>$ PD1(MP),PD2(MP),PD3,PD4,KB

C

REAL K0,KSQW(N), RHOB(N),CW(N), CB(N),FX1(N),FX2(N),FX3(N)

C THE COEFFICIENTS FOR THE RADIATION BOUNDARY CONDITION.

C

$\mathrm{PD} 3=0.2165063 * \mathrm{CI}$

$\mathrm{PD} 4=0.6856034 * \mathrm{CI}$

C

$\mathrm{KB}=\mathrm{CSQRT}(\mathrm{KSQB}(\mathrm{NZ}+2))$

$\mathrm{A} 1=1.0 / 6.0$

$\mathrm{A} 2=2.0 / 3.0$

$\mathrm{A} 3=1.0 / 6.0$

C

DO $1 \mathrm{I}=1, \mathrm{IZ}$

$\mathrm{FX1}(\mathrm{I})=1.0 / \mathrm{SQRT}(\mathrm{CW}(\mathrm{I}) / \mathrm{C0})$

$\mathrm{FX} 2(\mathrm{I})=1.0$

$\mathrm{FX3}(\mathrm{I})=\mathrm{SQRT}(\mathrm{CW}(\mathrm{I}) / \mathrm{C} 0)$

$\mathrm{KSQ}(\mathrm{I})=\mathrm{KSQW}(\mathrm{I})-\mathrm{K} 0 * * 2$

1 CONTINUE

C

DO $2 \mathrm{I}=\mathrm{IZ}+1, \mathrm{NZ}+2$

$\mathrm{FX} 1(\mathrm{I})=\mathrm{RHOB}(\mathrm{I}) / \mathrm{SQRT}(\mathrm{RHOB}(\mathrm{I}) * \mathrm{CB}(\mathrm{I}) / \mathrm{C} 0)$

$\mathrm{FX2}(\mathrm{I})=1.0 / \mathrm{RHOB}(\mathrm{I})$

$\mathrm{FX} 3(\mathrm{I})=\mathrm{SQRT}(\mathrm{RHOB}(\mathrm{I}) * \mathrm{CB}(\mathrm{I}) / \mathrm{C} 0)$

$\mathrm{KSQ}(\mathrm{I})=\mathrm{KSQB}(\mathrm{I})-\mathrm{K} 0 * * 2$

C

2 CONTINUE

DO $4 \mathrm{I}=1, \mathrm{NZ}$

$\mathrm{C} 1=(0.5 / \mathrm{DZ} * * 2) * \mathrm{FX} 1(\mathrm{I}+1) *(\mathrm{FX} 2(\mathrm{I})+\mathrm{FX} 2(\mathrm{I}+1)) * \mathrm{FX} 3(\mathrm{I})$

$\mathrm{C} 2=-(0.5 / \mathrm{DZ} * * 2) * \mathrm{FX} 1(\mathrm{I}+1) *(\mathrm{FX} 2(\mathrm{I})+2.0 * \mathrm{FX} 2(\mathrm{I}+1)+$

$>\mathrm{FX} 2(\mathrm{I}+2)) * \mathrm{FX} 3(\mathrm{I}+1)$

$\mathrm{C} 3=(0.5 / \mathrm{DZ} * * 2) * \mathrm{FX} 1(\mathrm{I}+1) *(\mathrm{FX} 2(\mathrm{I}+1)+\mathrm{FX} 2(\mathrm{I}+2)) * \mathrm{FX} 3(\mathrm{I}+2)$

$\mathrm{D} 1=\mathrm{C} 1+(\mathrm{KSQ}(\mathrm{I})+\mathrm{KSQ}(\mathrm{I}+1)) / 12.0$

$\mathrm{D} 2=\mathrm{C} 2+(\mathrm{KSQ}(\mathrm{I})+6.0 * \mathrm{KSQ}(\mathrm{I}+1)+\mathrm{KSQ}(\mathrm{I}+2)) / 12.0$

C

$\mathrm{D} 3=\mathrm{C} 3+(\mathrm{KSQ}(\mathrm{I}+1)+\mathrm{KSQ}(\mathrm{I}+2)) / 12.0$

DO $3 \mathrm{~J}=1$, NPADE

$\mathrm{E} 1=\mathrm{K} 0 * * 2 * \mathrm{~A} 1+\mathrm{PD} 2(\mathrm{~J}) * \mathrm{D} 1$

$\mathrm{E} 2=\mathrm{K} 0 * * 2 * \mathrm{~A} 2+\mathrm{PD} 2(\mathrm{~J}) * \mathrm{D} 2$

$\mathrm{E} 3=\mathrm{K} 0 * * 2 * \mathrm{~A} 3+\mathrm{PD} 2(\mathrm{~J}) * \mathrm{D} 3$

$\mathrm{F} 1=\mathrm{PD} 1(\mathrm{~J}) * \mathrm{CI} * \mathrm{~K} 0 * \mathrm{D} 1$

$\mathrm{F} 2=\mathrm{PD} 1(\mathrm{~J}) * \mathrm{CI} * \mathrm{~K} 0 * \mathrm{D} 2$

$\mathrm{F} 3=\mathrm{PD} 1(\mathrm{~J}) * \mathrm{CI} * \mathrm{~K} 0 * \mathrm{D} 3$

$\mathrm{R} 1(\mathrm{I}, \mathrm{J})=\mathrm{E} 1-0.5 * \mathrm{DR} * \mathrm{~F} 1$

$\mathrm{R} 2(\mathrm{I}, \mathrm{J})=\mathrm{E} 2-0.5 * \mathrm{DR} * \mathrm{~F} 2$

$\mathrm{R} 3(\mathrm{I}, \mathrm{J})=\mathrm{E} 3-0.5 * \mathrm{DR} * \mathrm{~F} 3$

$\mathrm{S} 1(\mathrm{I}, \mathrm{J})=\mathrm{E} 1+0.5 * \mathrm{DR} * \mathrm{~F} 1$

$\mathrm{S} 2(\mathrm{I}, \mathrm{J})=\mathrm{E} 2+0.5 * \mathrm{DR} * \mathrm{~F} 2$

$\mathrm{S} 3(\mathrm{I}, \mathrm{J})=\mathrm{E} 3+0.5 * \mathrm{DR} * \mathrm{~F} 3$

3 CONTINUE 


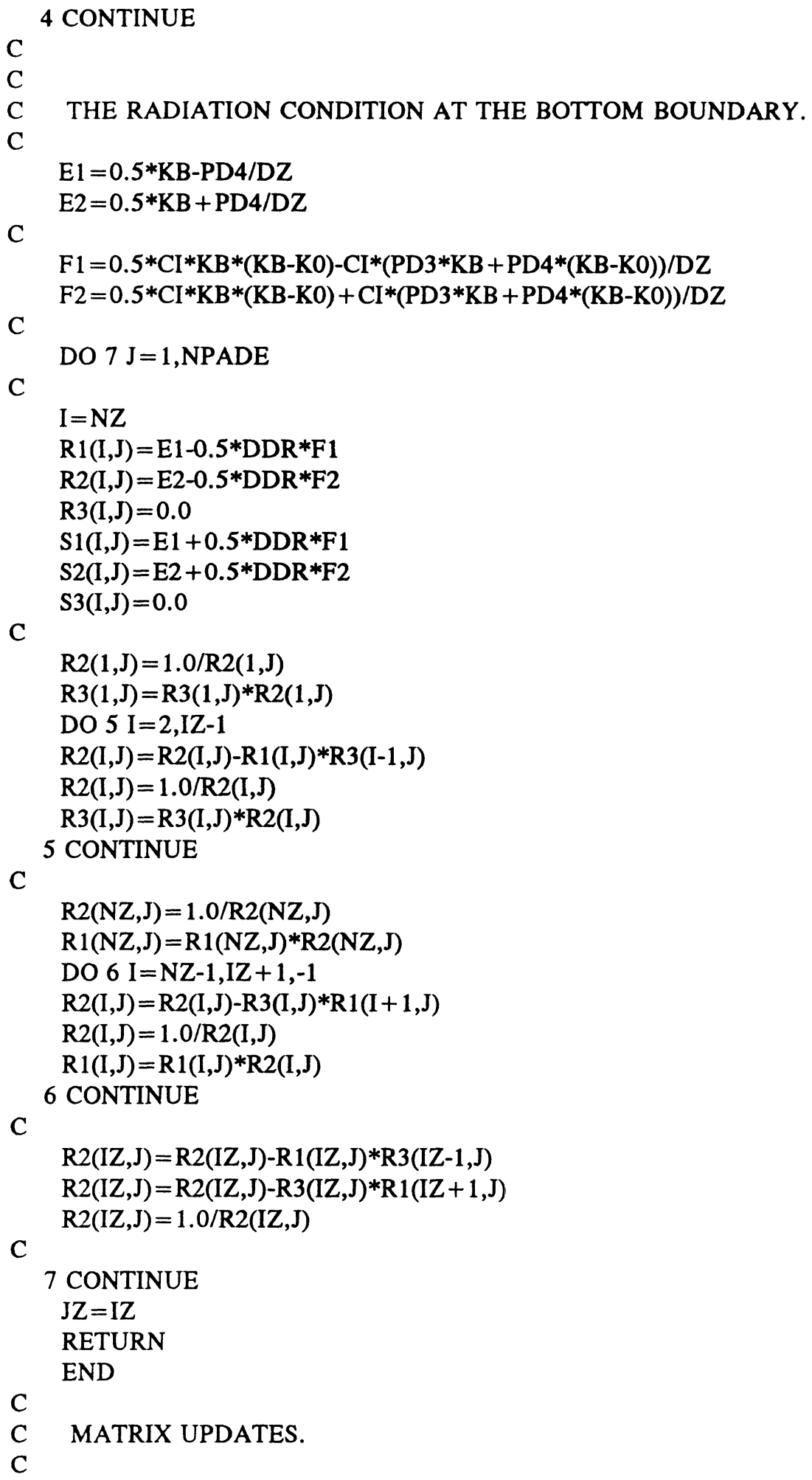


COMPLEX CI,D1,D2,D3,E1,E2,E3,F1,F2,F3, KSQB(N),KSQ(N),

$>\mathrm{R} 1(\mathrm{~N}, \mathrm{MP}), \mathrm{R} 2(\mathrm{~N}, \mathrm{MP}), \mathrm{R} 3(\mathrm{~N}, \mathrm{MP}), \mathrm{S} 1(\mathrm{~N}, \mathrm{MP}), \mathrm{S} 2(\mathrm{~N}, \mathrm{MP}), \mathrm{S} 3(\mathrm{~N}, \mathrm{MP})$,

$>$ PD1(MP),PD2(MP)

$\mathrm{C}$

REAL K0, KSQW(N), RHOB(N),CW(N), CB(N),FX1(N),FX2(N),FX3(N)

$\mathrm{A} 1=1.0 / 6.0$
$\mathrm{~A} 2=2.0 / 3.0$
$\mathrm{~A} 3=1.0 / 6.0$

$\mathrm{C}$

IF(IZ.GT.JZ)THEN

$\mathrm{I} 1=\mathrm{JZ}-1$

$\mathrm{I} 2=\mathrm{IZ}$

DO $1 \mathrm{I}=\mathrm{JZ}+1, \mathrm{IZ}$

$\mathrm{FX} 1(\mathrm{I})=1.0 / \mathrm{SQRT}(\mathrm{CW}(\mathrm{I}) / \mathrm{C} 0)$

$\mathrm{FX} 2(\mathrm{I})=1.0$

$\mathrm{FX} 3(\mathrm{I})=\mathrm{SQRT}(\mathrm{CW}(\mathrm{I}) / \mathrm{C} 0)$

$\mathrm{KSQ}(\mathrm{I})=\mathrm{KSQW}(\mathrm{I})-\mathrm{K} 0 * * 2$

1 CONTINUE

C

ELSE

$\mathrm{I} 1=\mathrm{IZ}-1$

$\mathrm{I} 2=\mathrm{JZ}$

DO $2 \mathrm{I}=\mathrm{IZ}+1, \mathrm{JZ}$

$\mathrm{FX} 1(\mathrm{I})=\mathrm{RHOB}(\mathrm{I}) / \mathrm{SQRT}(\mathrm{RHOB}(\mathrm{I}) * \mathrm{CB}(\mathrm{I}) / \mathrm{C} 0)$

$\mathrm{FX2}(\mathrm{I})=1.0 / \mathrm{RHOB}(\mathrm{I})$

$\mathrm{FX3}(\mathrm{I})=\mathrm{SQRT}(\mathrm{RHOB}(\mathrm{I}) * \mathrm{CB}(\mathrm{I}) / \mathrm{C} 0)$

$\mathrm{KSQ}(\mathrm{I})=\mathrm{KSQB}(\mathrm{I})-\mathrm{K} 0 * * 2$

2 CONTINUE

C

END IF

DO $4 \mathrm{I}=\mathrm{I} 1, \mathrm{I} 2$

$\mathrm{C} 1=(0.5 / \mathrm{DZ} * * 2) * \mathrm{FX} 1(\mathrm{I}+1) *(\mathrm{FX} 2(\mathrm{I})+\mathrm{FX} 2(\mathrm{I}+1)) * \mathrm{FX} 3(\mathrm{I})$

$\mathrm{C} 2=-(0.5 / \mathrm{DZ} * * 2) * \mathrm{FX} 1(\mathrm{I}+1) *(\mathrm{FX} 2(\mathrm{I})+2.0 * \mathrm{FX} 2(\mathrm{I}+1)+$

$>\mathrm{FX} 2(\mathrm{I}+2)) * \mathrm{FX} 3(\mathrm{I}+1)$

$\mathrm{C} 3=(0.5 / \mathrm{DZ} * * 2) * \mathrm{FX} 1(\mathrm{I}+1) *(\mathrm{FX} 2(\mathrm{I}+1)+\mathrm{FX} 2(\mathrm{I}+2)) * \mathrm{FX} 3(\mathrm{I}+2)$

$\mathrm{D} 1=\mathrm{C} 1+(\mathrm{KSQ}(\mathrm{I})+\mathrm{KSQ}(\mathrm{I}+1)) / 12.0$

$\mathrm{D} 2=\mathrm{C} 2+(\mathrm{KSQ}(\mathrm{I})+6.0 * \mathrm{KSQ}(\mathrm{I}+1)+\mathrm{KSQ}(\mathrm{I}+2)) / 12.0$

$\mathrm{D} 3=\mathrm{C} 3+(\mathrm{KSQ}(\mathrm{I}+1)+\mathrm{KSQ}(\mathrm{I}+2)) / 12.0$

C

DO $3 \mathrm{~J}=1$, NPADE

$\mathrm{E} 1=\mathrm{K} 0 * * 2 * \mathrm{~A} 1+\mathrm{PD} 2(\mathrm{~J}) * \mathrm{D} 1$

$\mathrm{E} 2=\mathrm{K} 0 * * 2 * \mathrm{~A} 2+\mathrm{PD} 2(\mathrm{~J}) * \mathrm{D} 2$

$\mathrm{E} 3=\mathrm{K} 0 * * 2 * \mathrm{~A} 3+\mathrm{PD} 2(\mathrm{~J}) * \mathrm{D} 3$

$\mathrm{F} 1=\mathrm{PD} 1(\mathrm{~J}) * \mathrm{CI} * \mathrm{~K} 0 * \mathrm{D} 1$

$\mathrm{F} 2=\mathrm{PD} 1(\mathrm{~J}) * \mathrm{CI} * \mathrm{~K} 0 * \mathrm{D} 2$

$\mathrm{F} 3=\mathrm{PD} 1(\mathrm{~J}) * \mathrm{CI} * \mathrm{~K} 0 * \mathrm{D} 3$

$\mathrm{R} 1(\mathrm{I}, \mathrm{J})=\mathrm{E} 1-0.5 * \mathrm{DR} * \mathrm{~F} 1$

$\mathrm{R} 2(\mathrm{I}, \mathrm{J})=\mathrm{E} 2-0.5 * \mathrm{DR} * \mathrm{~F} 2$

$\mathrm{R} 3(\mathrm{I}, \mathrm{J})=\mathrm{E} 3-0.5 * \mathrm{DR} * \mathrm{~F} 3$

$\mathrm{S} 1(\mathrm{I}, \mathrm{J})=\mathrm{E} 1+0.5 * \mathrm{DR} * \mathrm{~F} 1$

$\mathrm{S} 2(\mathrm{I}, \mathrm{J})=\mathrm{E} 2+0.5 * \mathrm{DR} * \mathrm{~F} 2$ 


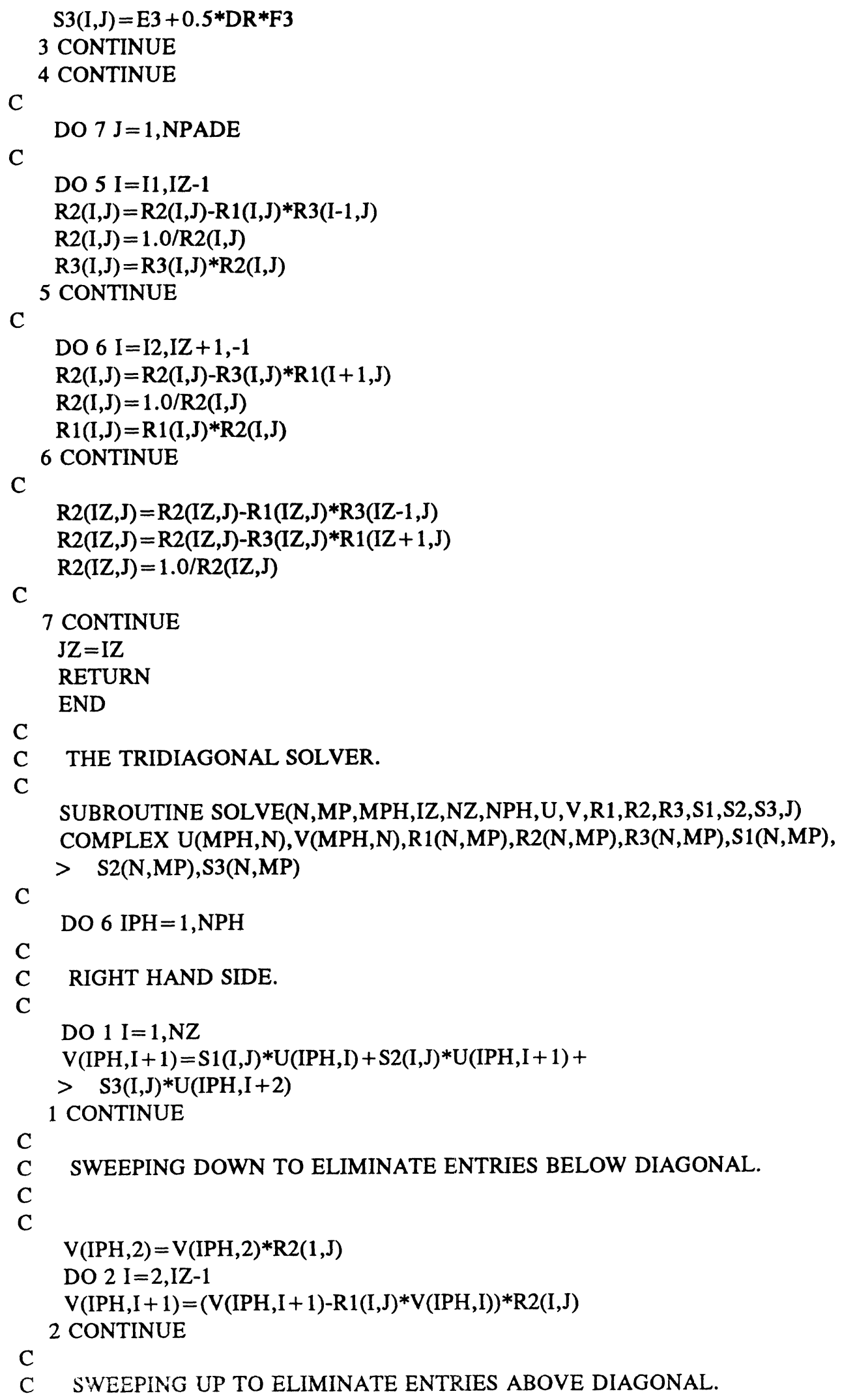




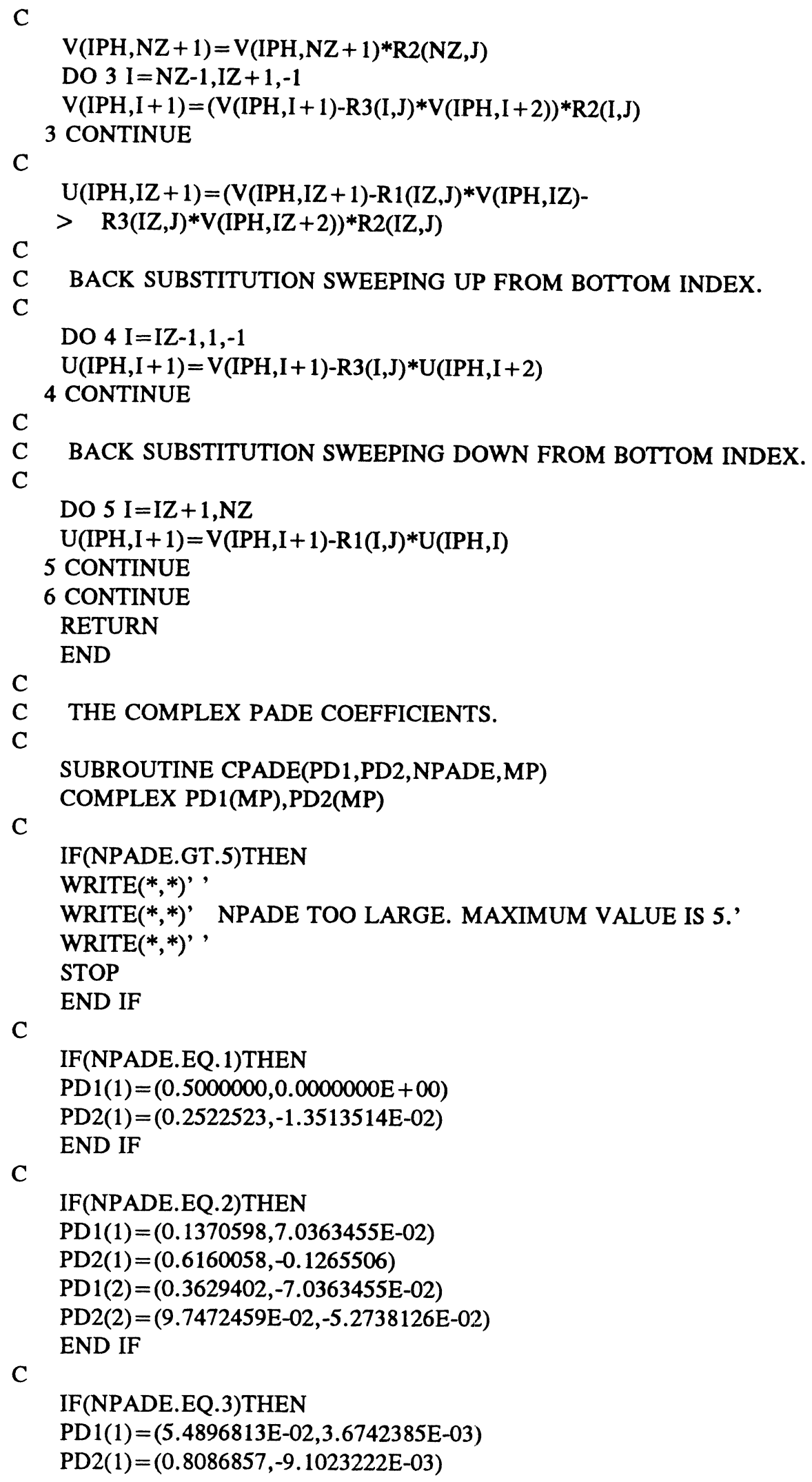


$\mathrm{PD} 1(2)=(0.1751380,3.1703257 \mathrm{E}-03)$

$\mathrm{PD} 2(2)=(0.3846456,-1.4611028 \mathrm{E}-02)$

$\mathrm{PD1}(3)=(0.2699652,-6.8445643 \mathrm{E}-03)$

$\mathrm{PD} 2(3)=(4.8821609 \mathrm{E}-02,-2.9557836 \mathrm{E}-03)$

END IF

C

IF(NPADE.EQ.4)THEN

PD1 $(1)=(2.5381334 \mathrm{E}-02,6.0041733 \mathrm{E}-03)$

$\mathrm{PD} 2(1)=(0.8832191,-1.8850777 \mathrm{E}-02)$

$\mathrm{PD} 1(2)=(8.7361716 \mathrm{E}-02,9.9593531 \mathrm{E}-03)$

$\mathrm{PD} 2(2)=(0.5945545,-4.3388147 \mathrm{E}-02)$

$\mathrm{PD1}(3)=(0.1649370,-2.0355983 \mathrm{E}-03)$

$\mathrm{PD} 2(3)=(0.2610570,-3.0401150 \mathrm{E}-02)$

$\mathrm{PD} 1(4)=(0.2223199,-1.3927928 \mathrm{E}-02)$

$\mathrm{PD} 2(4)=(3.2206986 \mathrm{E}-02,-4.3234974 \mathrm{E}-03)$

C

END IF

IF(NPADE.EQ.5)THEN

$\mathrm{PD} 1(1)=(1.6475746 \mathrm{E}-02,2.6173585 \mathrm{E}-03)$

$\mathrm{PD} 2(1)=(0.9128319,-9.0011684 \mathrm{E}-03)$

$\mathrm{PD} 1(2)=(5.7667062 \mathrm{E}-02,8.8840965 \mathrm{E}-03)$

$\mathrm{PD} 2(2)=(0.6861078,-3.0282630 \mathrm{E}-02)$

$\mathrm{PD} 1(3)=(0.1035779,1.0283243 \mathrm{E}-02)$

$\mathrm{PD} 2(3)=(0.4076422,-4.4838917 \mathrm{E}-02)$

$\mathrm{PD} 1(4)=(0.1452988,-4.1513294 \mathrm{E}-03)$

$\mathrm{PD} 2(4)=(0.1658151,-2.9934738 \mathrm{E}-02)$

$\mathrm{PD} 1(5)=(0.1769805,-1.7633369 \mathrm{E}-02)$

$\mathrm{PD} 2(5)=(1.9913642 \mathrm{E}-02,-4.2300178 \mathrm{E}-03)$

C

END IF

RETURN

END

C

C THE ENERGY IS EVALUATED.

C

SUBROUTINE ENERGY(UREP,UDAT,NPH,E)

C COMPLEX UREP(100),UDAT(100),RD

$$
\begin{aligned}
& \mathrm{AMP}=0.0 \\
& \mathrm{RD}=\mathrm{CMPLX}(0.0,0.0)
\end{aligned}
$$

DO $1 \mathrm{IPH}=1, \mathrm{NPH}$

$\mathrm{AMP}=\mathrm{AMP}+\mathrm{CABS}(\mathrm{UREP}(\mathrm{IPH})) * * 2$

$\mathrm{RD}=\mathrm{RD}+\mathrm{UREP}(\mathrm{IPH}) * \mathrm{UDAT}(\mathrm{IPH})$

1 CONTINUE

C

$\mathrm{E}=\mathrm{CABS}(\mathrm{RD}) * * 2 / \mathrm{AMP}$

$$
\begin{aligned}
& \text { RETURN } \\
& \text { END }
\end{aligned}
$$

C

C THE ENITRONMENT IS PARMETRIZED.

C 
SUBROUTINE PARMTR(F,G,NF,CW,CB, RHOB,ATTN,N)

C REAL CW(N),CB(N), RHOB(N), ATTN(N),F(100), G(100)

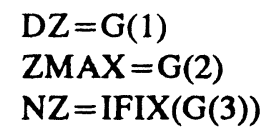


C

RETURN

END

C

SUBROUTINE PIVOT(A,B,I,N)

COMPLEX*16 A(100,100),B(100,100),TEMP

$\mathrm{J}=\mathrm{I}$

$\operatorname{PMAX}=\operatorname{CDABS}(\mathrm{A}(\mathrm{I}, \mathrm{I}))$

DO $1 \mathrm{~K}=\mathrm{I}+1, \mathrm{~N}$

PNEW $=$ CDABS $(A(K, I))$

IF(PNEW.GT.PMAX)THEN

$\mathrm{J}=\mathrm{K}$

PMAX $=$ PNEW

END IF

1 CONTINUE

C

IF(I.EQ.J)RETURN

DO $2 \mathrm{~K}=\mathrm{I}, \mathrm{N}$

TEMP $=\mathrm{A}(\mathrm{J}, \mathrm{K})$

$\mathrm{A}(\mathrm{J}, \mathrm{K})=\mathrm{A}(\mathrm{I}, \mathrm{K})$

$\mathrm{A}(\mathrm{I}, \mathrm{K})=\mathrm{TEMP}$

$\mathrm{TEMP}=\mathrm{B}(\mathrm{J}, \mathrm{K})$

$\mathrm{B}(\mathrm{J}, \mathrm{K})=\mathrm{B}(\mathrm{I}, \mathrm{K})$

$\mathrm{B}(\mathrm{I}, \mathrm{K})=\mathrm{TEMP}$

2 CONTINUE

RETURN

END

C

SUBROUTINE MULT(A,B,I,N)

C

COMPLEX*16 A(100,100),B(100,100),FACT

$\mathrm{FACT}=1.0 / \mathrm{A}(\mathrm{I}, \mathrm{I})$

DO $1 \mathrm{~J}=\mathrm{I}, \mathrm{N}$

$\mathrm{A}(\mathrm{I}, \mathrm{J})=\mathrm{A}(\mathrm{I}, \mathrm{J}) * \mathrm{FACT}$

1 CONTINUE

DO $2 \mathrm{~J}=1, \mathrm{~N}$

$\mathrm{B}(\mathrm{I}, \mathrm{J})=\mathrm{B}(\mathrm{I}, \mathrm{J}) * \mathrm{FACT}$

2 CONTINUE

RETURN

END

C

SUBROUTINE ADD1(A,B,I,N)

C

COMPLEX*16 A(100,100),B(100,100),FACT

DO $3 \mathrm{~J}=\mathrm{I}+1, \mathrm{~N}$

$\mathrm{FACT}=\mathrm{A}(\mathrm{J}, \mathrm{I})$

$\mathrm{A}(\mathrm{J}, \mathrm{I})=0.0$

DO $1 \mathrm{~K}=\mathrm{I}+1, \mathrm{~N}$

$\mathrm{A}(\mathrm{J}, \mathrm{K})=\mathrm{A}(\mathrm{J}, \mathrm{K})-\mathrm{FACT} * \mathrm{~A}(\mathrm{I}, \mathrm{K})$

1 CONTINUE

DO $2 \mathrm{~K}=1, \mathrm{~N}$ 
$\mathrm{B}(\mathrm{J}, \mathrm{K})=\mathrm{B}(\mathrm{J}, \mathrm{K})-\mathrm{FACT} * \mathrm{~B}(\mathrm{I}, \mathrm{K})$

2 CONTINUE

3 CONTINUE

RETURN

END

C

SUBROUTINE ADD2(A,B,I,N)

COMPLEX*16 A(100,100),B(100,100)

DO $2 \mathrm{~J}=1, \mathrm{I}-1$

DO $1 \mathrm{~K}=1, \mathrm{~N}$

$\mathrm{B}(\mathrm{J}, \mathrm{K})=\mathrm{B}(\mathrm{J}, \mathrm{K})-\mathrm{A}(\mathrm{J}, \mathrm{I}) * \mathrm{~B}(\mathrm{I}, \mathrm{K})$

1 CONTINUE

2 CONTINUE

RETURN

END 


\section{APPENDIX G Bartlett and MLM Ambiguity Surface Display Code}

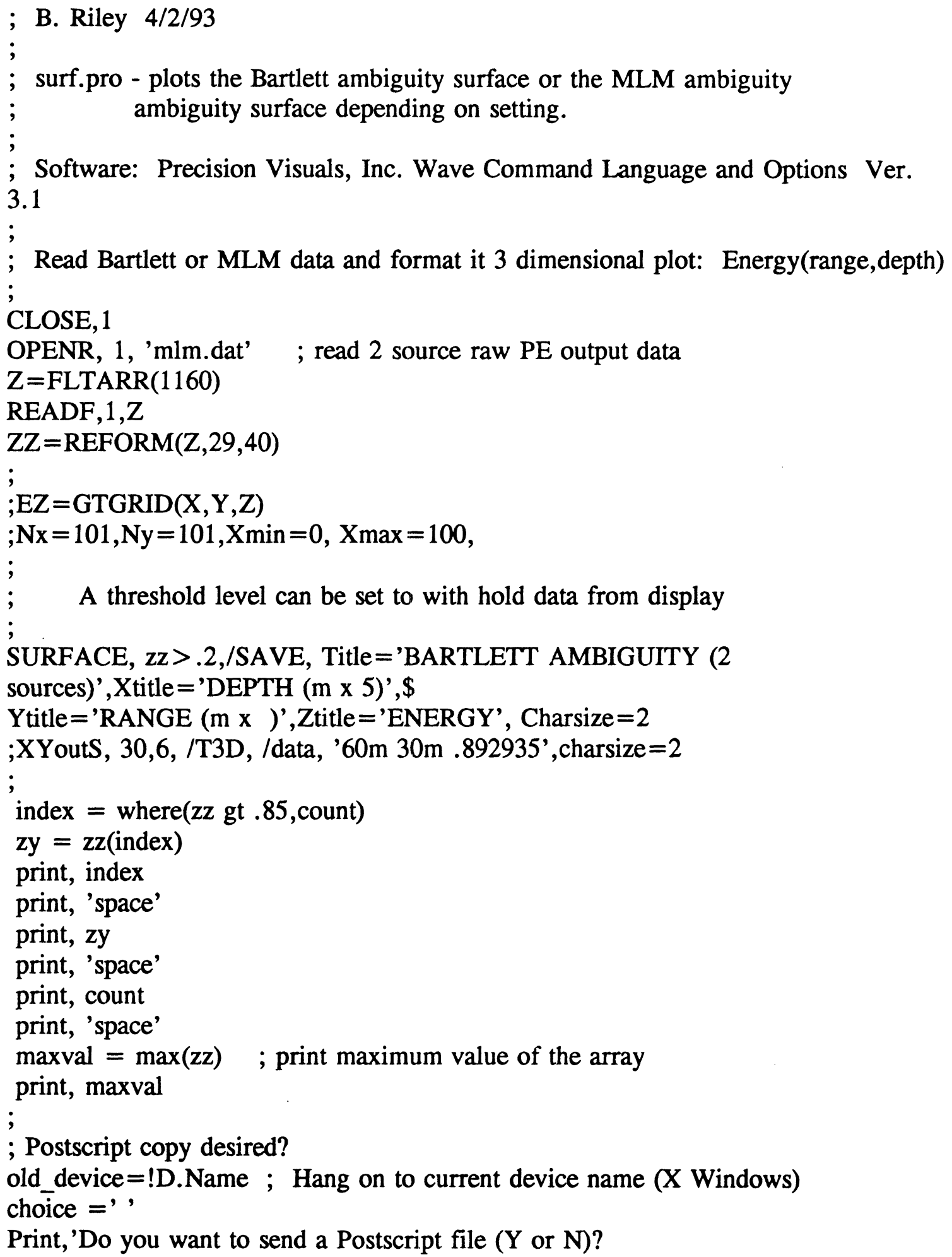


choice $=$ Get_KBRD(1) ; Wait for a single character from the keyboard.

;

IF (choice EQ 'y' ) or (choice EQ 'Y') Then Begin

Print, 'Printing PostScritp Graphics.'

Set_Plot, 'PS'; Set to Postscript device.

Device, Filename $=$ 'postscript.hpgl'

Device, /Landscape ; Optional - the default is portrait.

Surface, ZZ, /SAVE, Title='MLM AMBIGUITY (source \&

jammer)',Xtitle ='DEPTH $(\mathrm{m} \times 5)$ ',Ytitle='RANGE $(\mathrm{m} \times 10)$ '\$

,Ztitle = 'ENERGY', Charsize $=2$

; XYoutS, 30,6, /T3D, /data, '60m 30m .892935', charsize $=2$

Device, /close ; Close the Postscript output file.

Spawn, 'lpr postscript.ps' ; This is optional - it send the file to print. ENDIF

Set_Plot, old_device ; Sets plotting back to screen

END 


\section{APPENDIX H GLRT Computational and Display Code}

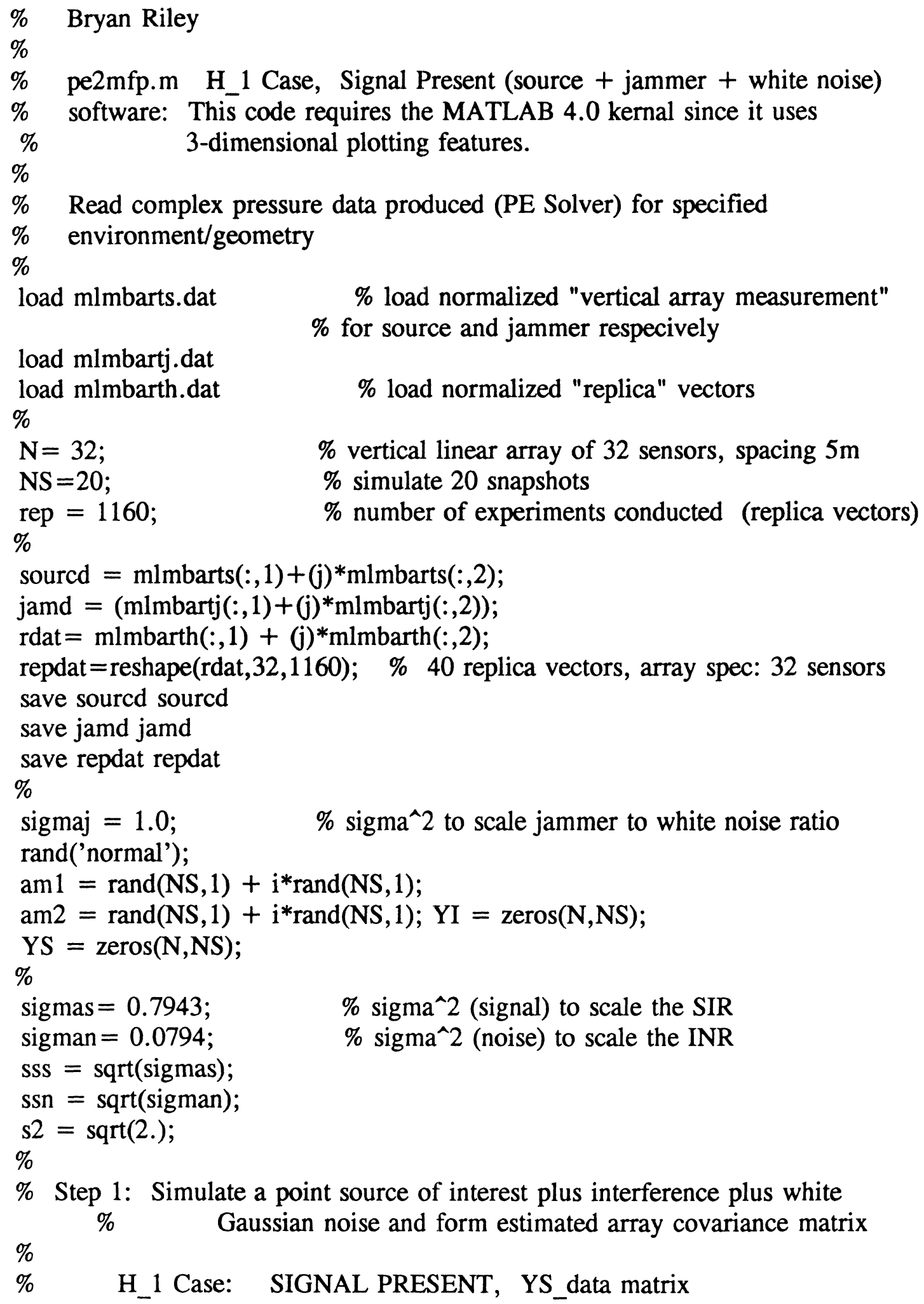




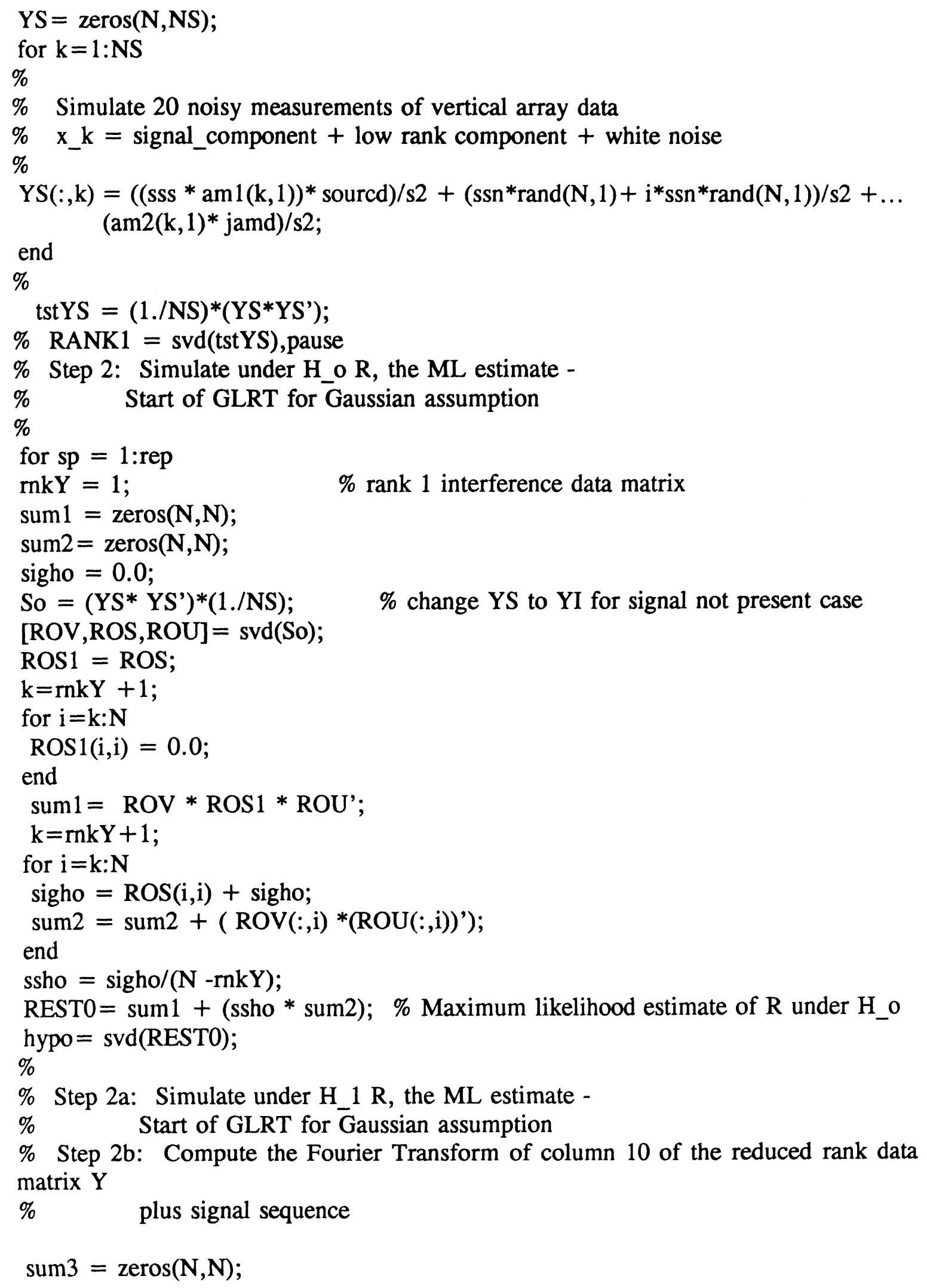




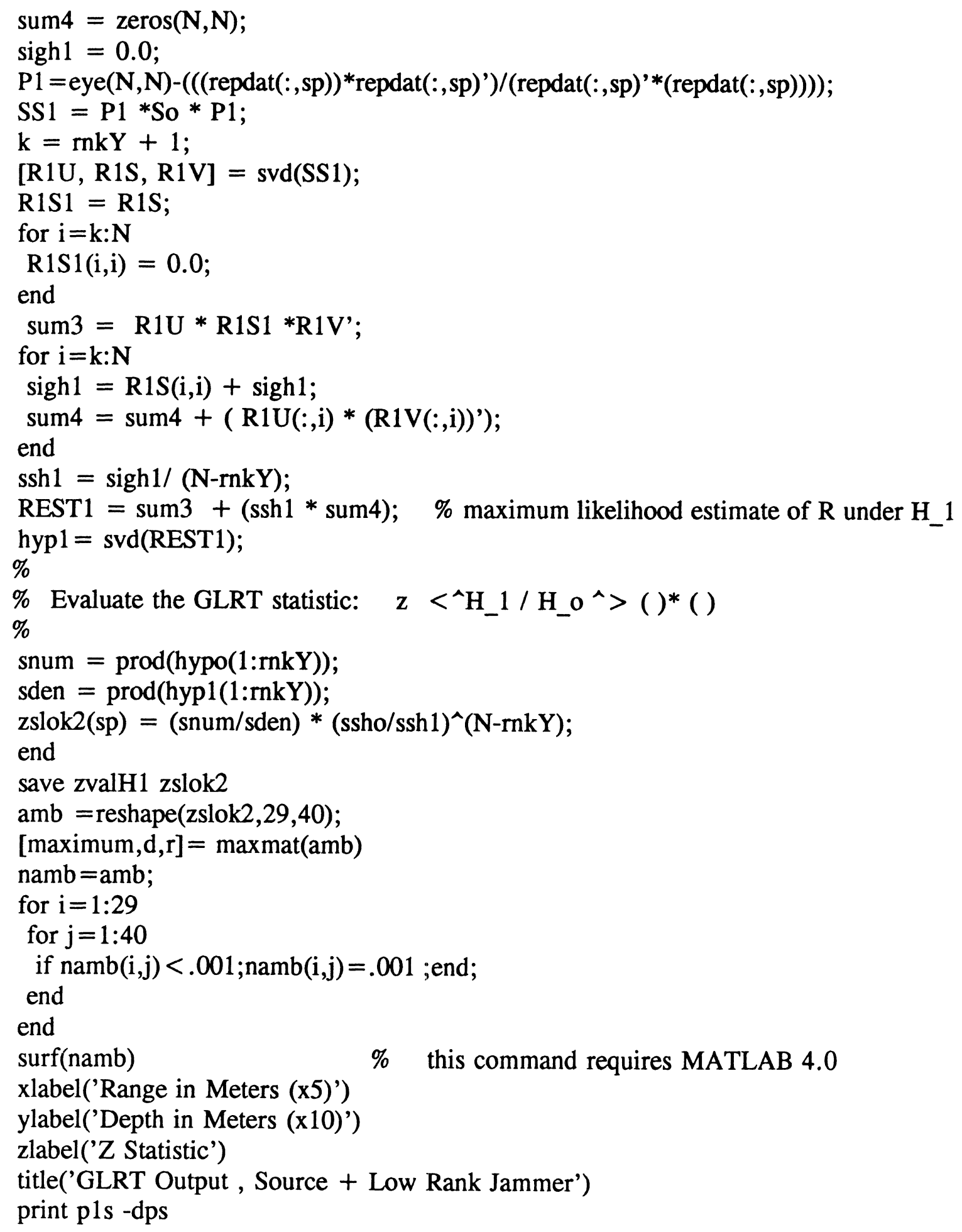




\begin{abstract}
RILEY, H. BRYAN, Ph.D. March, 1994. Electrical and Computer Engineering

Matched-Field Source Detection and Localization In High Noise Environments: A Novel Reduced Rank Signal Processing Approach (176pp.)
\end{abstract}

Director of Dissertation: Dr. John A. Tague

This dissertation describes a new matched-field acoustic source localization technique using reduced-rank signal processing. We pose the problem as joint detection and parameter estimation in the face of unknown, structured noise. We derive a reducedrank likelihood ratio array processor and demonstrate its ability to detect and localize sources of interest. A key feature of reduced-rank signal processing is the ability to discard undesirable information from data matrices by their low rank approximation.

The basis of this investigation is formulated with the normal mode model and Parabolic Equation method to model wavefront curvature and model conditions in complex ocean environments. The problem is formulated based upon a receiving array measures signals in the form of uncorrelated elementary waves. This work models the acoustic wavefields as they propagate through nonhomogeneous media and includes range dependent characteristics. Insight is gained by implementing the Bartlett and maximum likelihood estimators for a specified problem geometry and ocean environmental conditions. This work is presents the GLRT which incorporates ideas from linear algebra, multivariate statistics, and reduced rank signal processing. This new 